\title{
Development of a Task Analysis Tool to Estimate Shoulder Strain During a Lifting Task
}

Christopher Wayne Moore

cmoore18@mix.wvu.edu

Follow this and additional works at: https://researchrepository.wvu.edu/etd

Part of the Ergonomics Commons

\section{Recommended Citation}

Moore, Christopher Wayne, "Development of a Task Analysis Tool to Estimate Shoulder Strain During a Lifting Task" (2019). Graduate Theses, Dissertations, and Problem Reports. 3942.

https://researchrepository.wvu.edu/etd/3942

This Dissertation is protected by copyright and/or related rights. It has been brought to you by the The Research Repository @ WVU with permission from the rights-holder(s). You are free to use this Dissertation in any way that is permitted by the copyright and related rights legislation that applies to your use. For other uses you must obtain permission from the rights-holder(s) directly, unless additional rights are indicated by a Creative Commons license in the record and/ or on the work itself. This Dissertation has been accepted for inclusion in WVU Graduate Theses, Dissertations, and Problem Reports collection by an authorized administrator of The Research Repository @ WVU.

For more information, please contact researchrepository@mail.wvu.edu. 


\title{
DEVELOPMENT OF A TASK ANALYSIS TOOL TO ESTIMATE SHOULDER STRAIN DURING A LIFTING TASK
}

\author{
Christopher W. Moore, MS
}

Dissertation submitted to the Benjamin M. Statler College of Engineering and Mineral Resources at West Virginia University in partial fulfillment of the requirement for the degree of

Doctor of Philosophy in Occupational Safety and Health

Ashish D. Nimbarte, Ph.D., Chair Kevin He, Ph.D. Hongwei Hsiao, Ph.D. Xiaopeng Ning, Ph.D. Gary Winn, Ph.D.

\author{
Department of Industrial and Management Systems Engineering \\ West Virginia University \\ Morgantown, West Virginia \\ 2019
}

Keywords: Musculoskeletal disorders, concavity-compression mechanism, predictive equation, task analysis, shoulder joint

Copyright 2019 - Christopher W. Moore, MS 


\title{
ABSTRACT \\ DEVELOPMENT OF A TASK ANALYSIS TOOL TO ESTIMATE SHOULDER STRAIN DURING A LIFTING TASK
}

\author{
Christopher W. Moore, MS
}

Musculoskeletal disorders of the shoulder have a huge impact on the workplace and employees. Due to the complexity and high mobility of the shoulder, developing a task analysis tool with a focus on the shoulder is difficult, and while there are some ergonomic analysis tools that do consider the shoulder to varying degrees in the analysis, none focus purely on the shoulder. Therefore, this research was undertaken in an attempt to develop a shoulder-based task analysis tool for the shoulder. However, since the scope of work involving the shoulder is so vast, the tool in this research focuses on unobstructed, one-handed lifting tasks.

An initial evaluation utilizing correlation and root mean square error analysis was performed using motion capture and electromyography data from participants performing a lifting task with dynamic and static portions using 3 different weights. Each task was modeled in AnyBody Modeling System with each available muscle recruitment algorithm. Based on correlation and root mean square error analysis between the muscle activations from the model and the collected electromyography data, the Min/Max strategy was most appropriate for static tasks and Poly4 strategy for static exertions. These selected muscle recruitment algorithms were used to model the tasks performed by the participants through the other sections of this research.

Next, five participants performed static lifting tasks supporting a 15-pound weight throughout the reach zone of the right arm. These tasks were modeled in AnyBody using the Min/Max recruitment algorithm based on the results of the previous aim. Twelve potential composite index equations, designed to estimate shoulder strain based on AnyBody model outputs, were analyzed, three equations developed for this research and one previously-developed and validated equation. Correlation analysis between the results of each potential composite index and perceived exertions led toward the selection of one of the newly developed composite index equations, though the results were so close that the previously-developed equation was selected. Regression methods were used to develop a regression equation to predict the composite index values based on the distance to the load from the sternal notch.

Finally, the hypothesis that the strain of an unobstructed lifting task would be highest at either the origin or the destination of the lift was tested to determine if the strain on the shoulder for a lifting task could be estimated based on the origin and destination of the load. Five participants were recruited to perform lifting tasks between low-, medium-, and high-risk load locations, and the peak predicted strain throughout the trial was compared to the strain at the origin and destination. It was determined that, due to an initial lift from the origin accompanied by an arced lifting trajectory, the peak strain was often slightly higher than at the higher of the origin or destination. 


\section{Table of Contents}

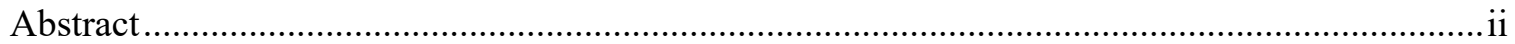

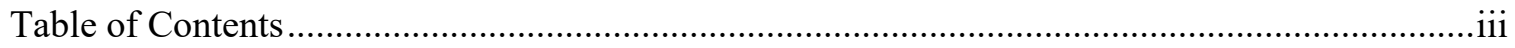

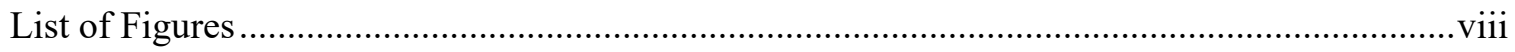

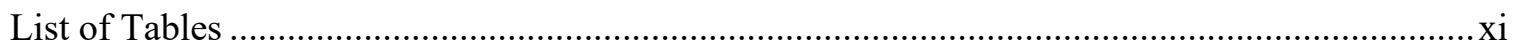

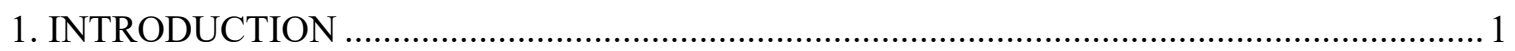

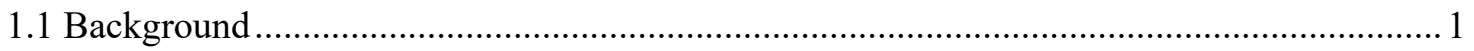

1.1.1 Shoulder Musculoskeletal Disorder Prevalence and Cost .......................................... 1

1.1.2 Ergonomic Analysis Methods of the Shoulder .......................................................... 2

1.1.3 Risk Factors for Shoulder Musculoskeletal Disorders .................................................. 3

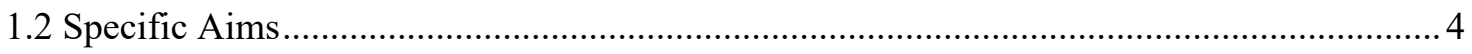

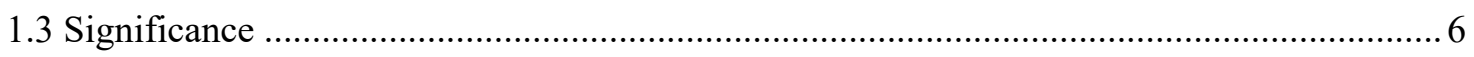

2. SPECIFIC AIM \#1: MUSCLE RECRUITMENT STRATEGY DETERMINATION .............. 7

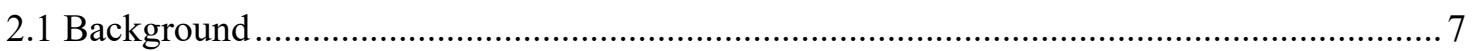

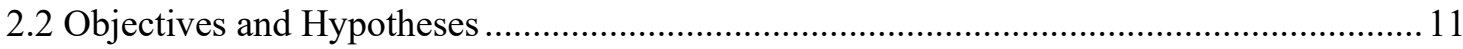

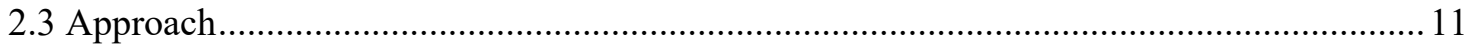

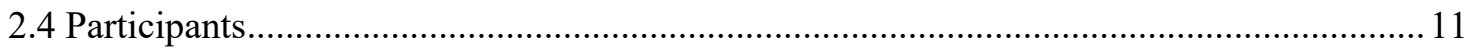

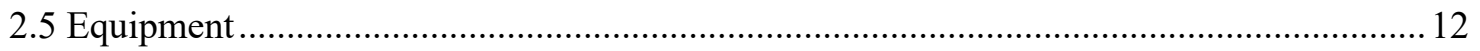

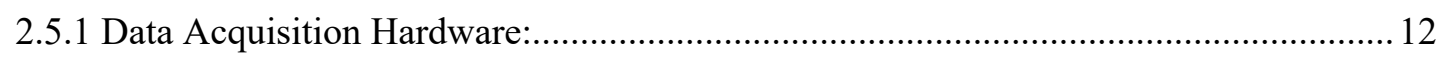

2.5.1.1 Optical Motion Capture System (MX Series, Vicon Motion Sys, Oxford, UK): .. 12

2.5.1.2 Electromyography System (Bagnoli-16, Delsys Inc., Natick, MA, USA): ........... 13

2.5.2 Rehabilitation Machine (Humac Norm, CSMi Solutions, Stoughton, MA, USA):...... 15

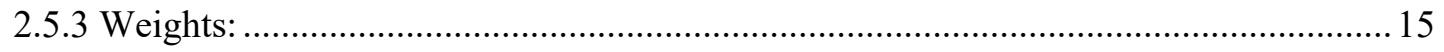

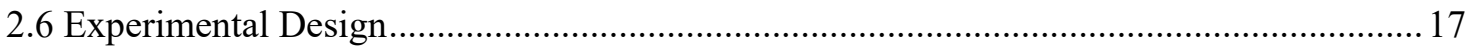

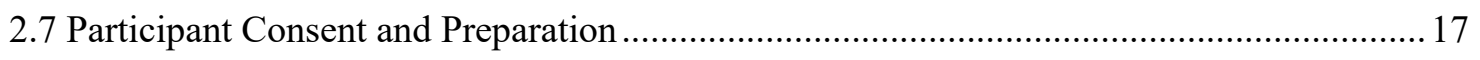

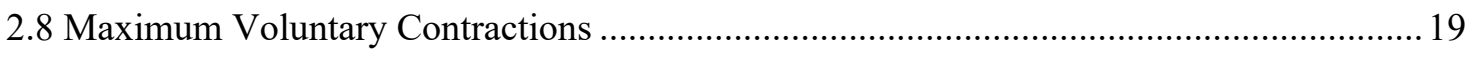

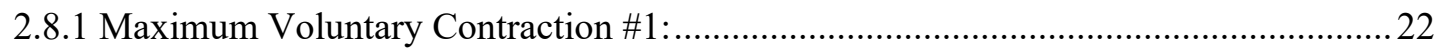




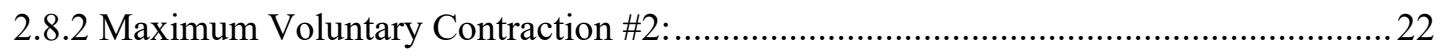

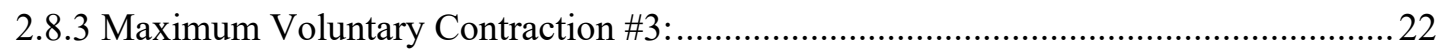

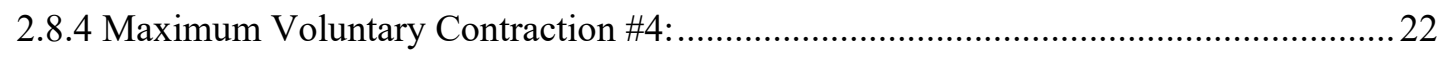

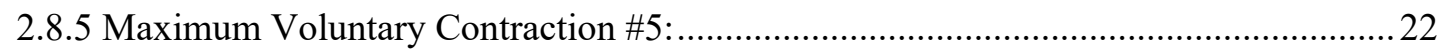

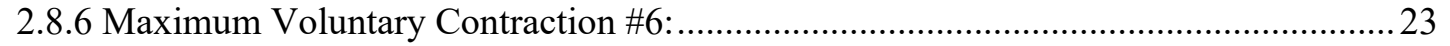

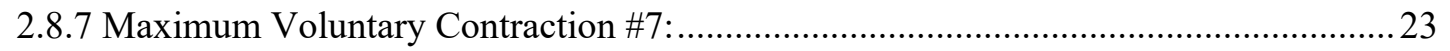

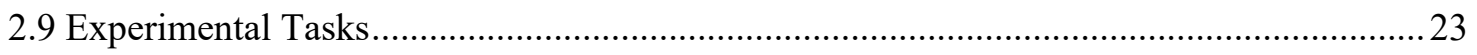

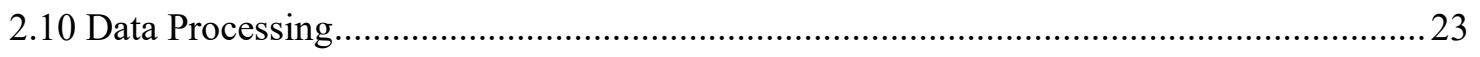

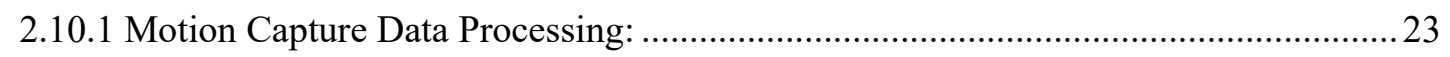

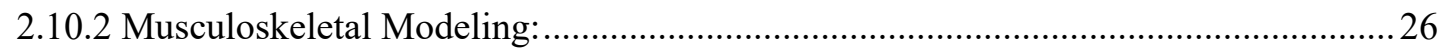

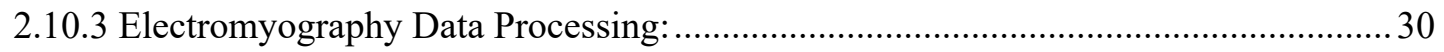

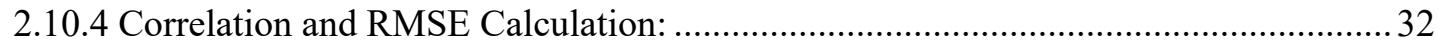

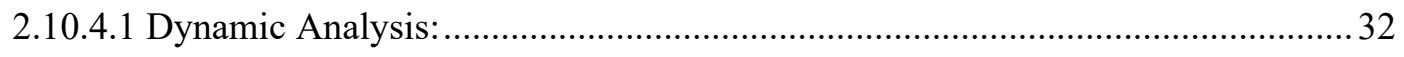

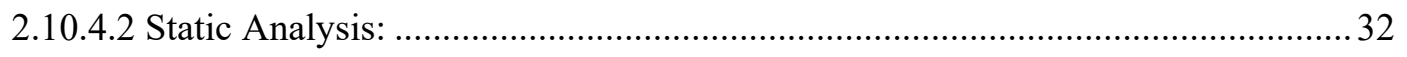

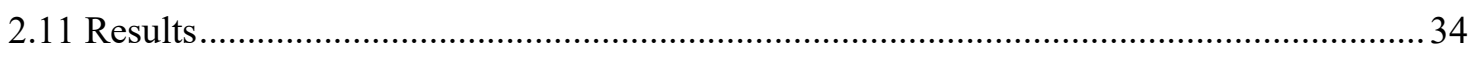

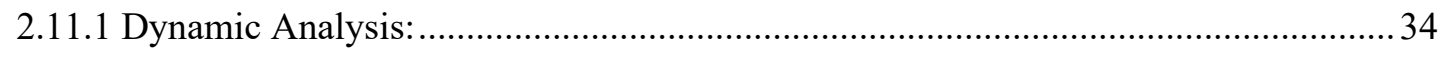

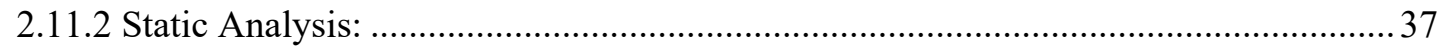

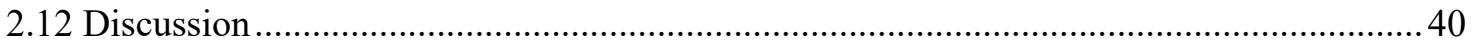

3. SPECIFIC AIM \#2: COMPOSITE INDEX AND PREDICTIVE EQ. DEVELOPMENT ....... 41

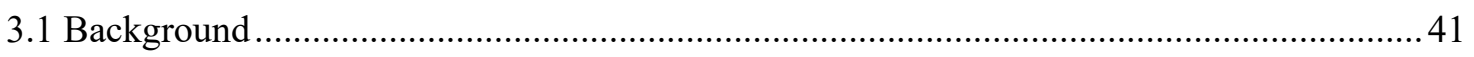

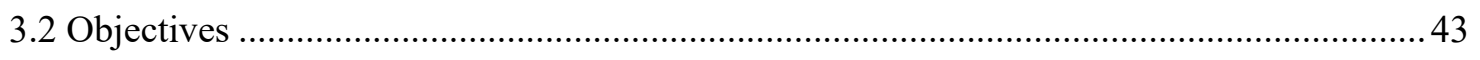

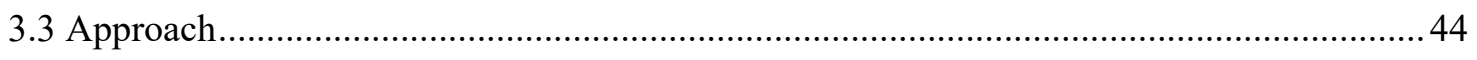

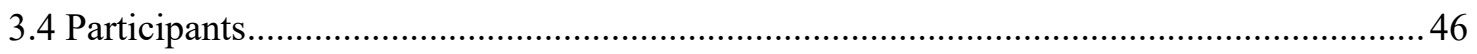

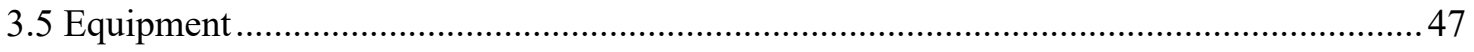

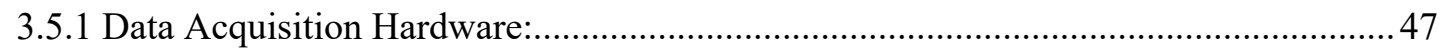

3.5.2 Hydraulic Scissor Lift (Vestil EHLT-4872-3-43, Vestil Mfg., Angola, IN, USA): ..... 47

3.5.3 Custom Weight Support and Floor Grid: ........................................................... 47 


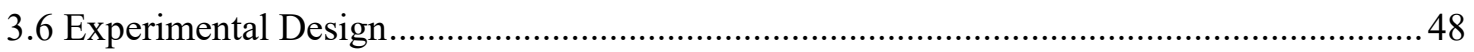

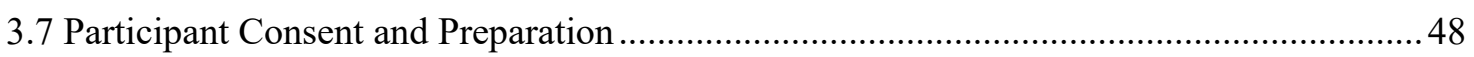

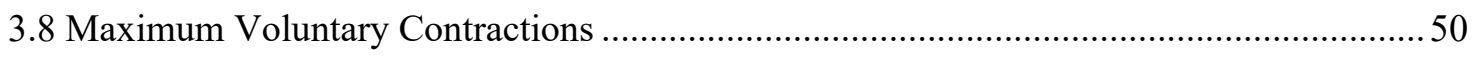

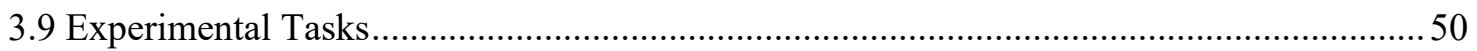

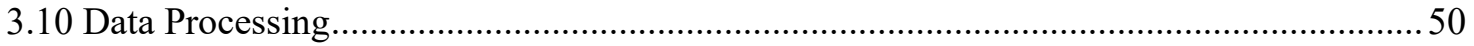

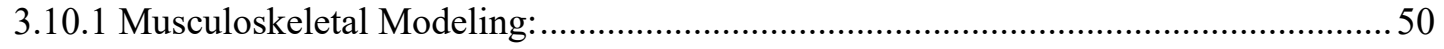

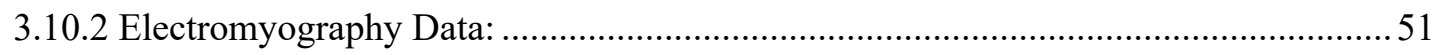

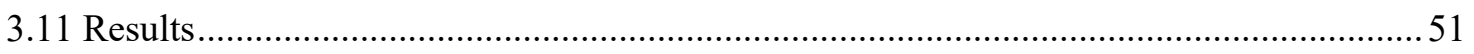

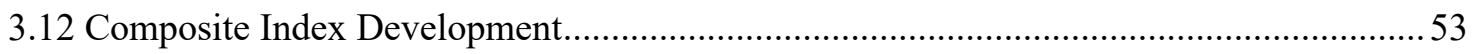

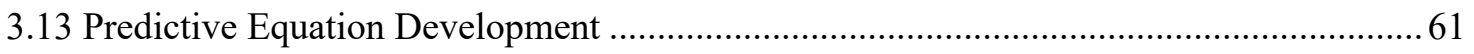

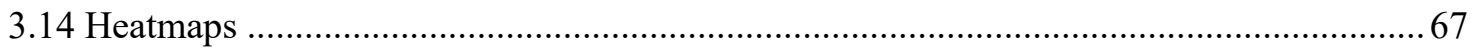

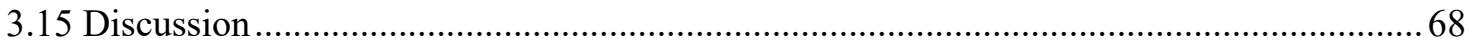

4. SPECIFIC AIM \#3: VALIDATION OF PREDICTIVE EQ. AS TASK ANALYSIS TOOL .. 75

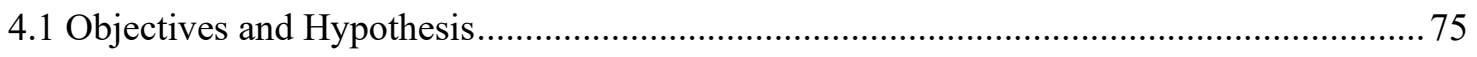

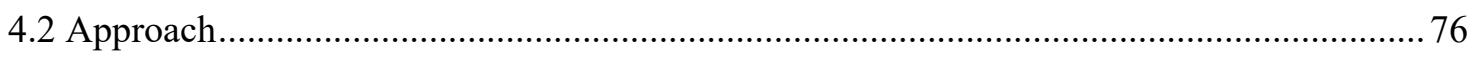

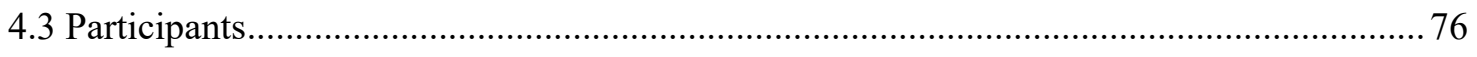

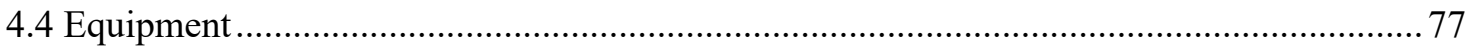

4.4.1 Optical Motion Capture System (MX Series, Vicon Motion Systems, Oxford, UK):.. 77

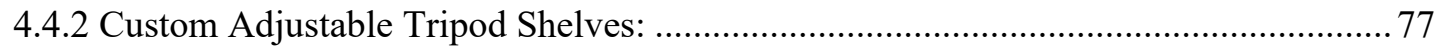

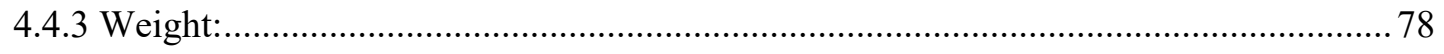

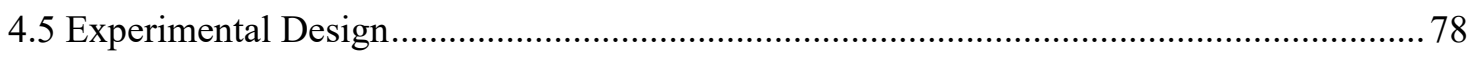

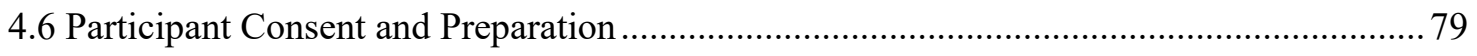

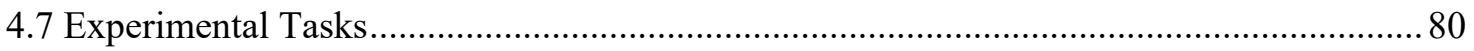

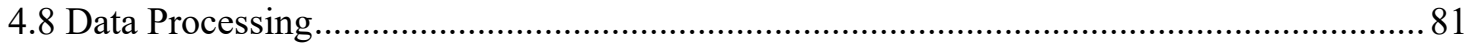

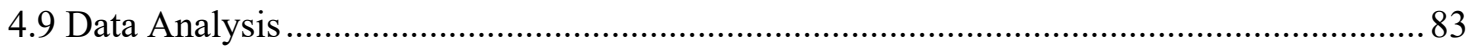

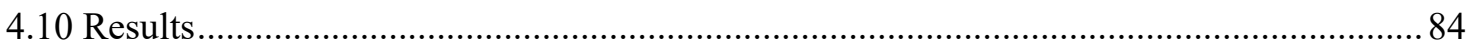

4.10.1 Further Validation of Predictive Equation: ............................................................. 84 


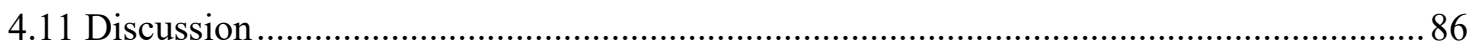

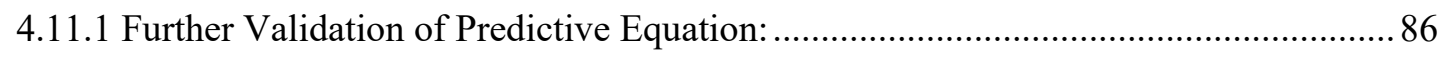

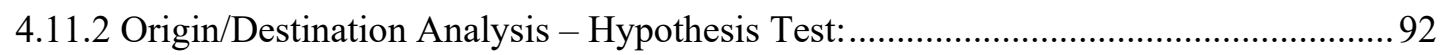

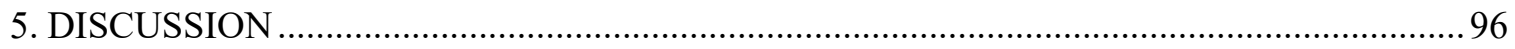

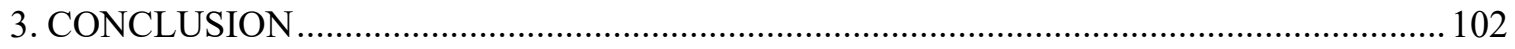

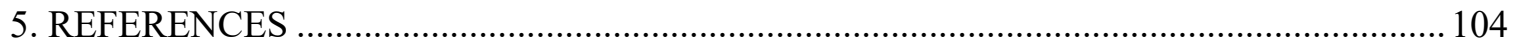

Appendix A: Literature Review of Shoulder Musculoskeletal Disorder Risk Factors ................ 109

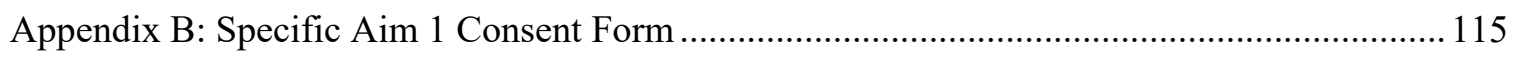

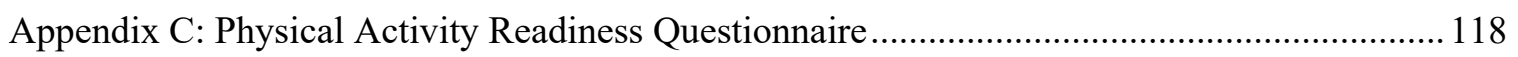

Appendix D: Maximum Voluntary Contraction Guidesheets................................................. 119

Appendix E: MATLAB Codes for Specific Aim 1 Data Processing ......................................... 127

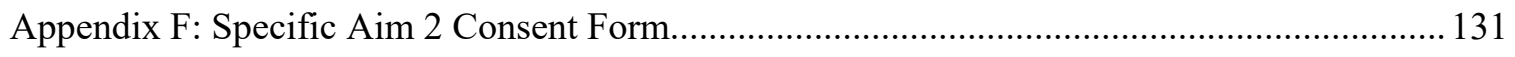

Appendix G: Borg CR-10 Rating of Perceived Exertion Scale .............................................. 135

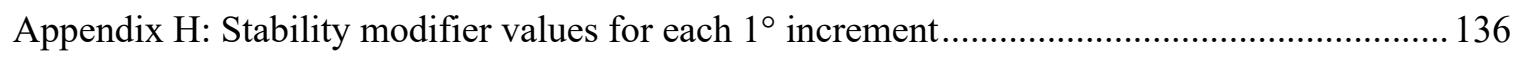

Appendix I: Best Sets Regression Analysis of 11 Potential Sets of Predictors ......................... 140

Appendix J1: Minitab Output from Regression Analysis (Regression Equation \#1) .................. 146

Appendix J2: Minitab Output from Regression Analysis (Regression Equation \#2) .................. 148

Appendix J3: Minitab Output from Regression Analysis (Regression Equation \#3) .................. 149

Appendix J4: Minitab Output from Regression Analysis (Regression Equation \#4) .................. 150

Appendix K1. Predicted Strain with the Load 20 Inches Above Sternal Notch......................... 151

Appendix K2. Predicted Strain with the Load 15 Inches Above Sternal Notch......................... 152

Appendix K3. Predicted Strain with the Load 10 Inches Above Sternal Notch.......................... 153

Appendix K4. Predicted Strain with the Load 5 Inches Above Sternal Notch. ........................... 154

Appendix K5. Predicted Strain with the Load Horizontally Aligned with Sternal Notch........... 155

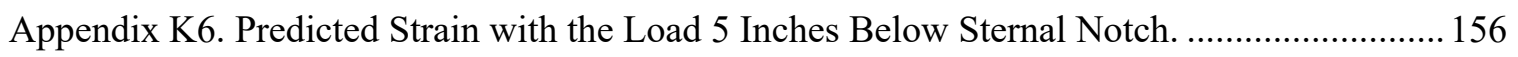

Appendix K7. Predicted Strain with the Load 10 Inches Below Sternal Notch. ........................ 157 
Appendix K8. Predicted Strain with the Load 15 Inches Below Sternal Notch. ...

Appendix K9. Predicted Strain with the Load 20 Inches Below Sternal Notch. .

Appendix L: Specific Aim 3 Consent Form 


\section{List of Figures}

Figure 1. Model View of the modified MoCap-FullBody model that was used throughout this research... 8

Figure 2. Schematic of the physical connectivity of the data acquisition hardware ................................. 13

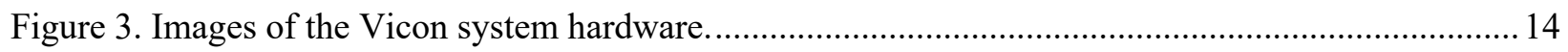

Figure 4. Components of the Delsys Bagnoli electromyography system ............................................ 15

Figure 5. Rehabilitation machine used during maximum voluntary exertions .......................................... 16

Figure 6. D-handle bolted to four flat weights weighing 2.5 pounds each, resulting in 10-pound weight. 16

Figure 7. Set of 32 retro-reflective markers in the marker set attached to a participant.......................... 18

Figure 8. Set of 13 electromyography sensors attached superficial to muscles of interest....................... 19

Figure 9. Progression for the experimental task performed by the participants. .....................................2 24

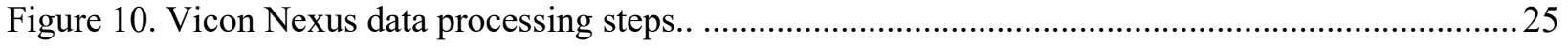

Figure 11. Musculotendon units comprising the Deltoid muscle .....................................................2

Figure 12. Electromyography data processing steps. (a) Raw electromyography data. (b) Notch filtered and demeaned data. (c) Full-wave rectified data. (d) Butterworth filtered data forming a linear

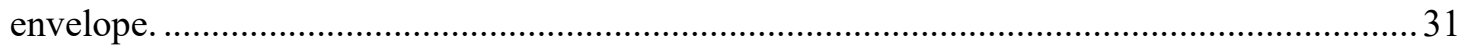

Figure 13. Mean correlation values and mean RMSE values found for each recruitment strategy for the dynamic analysis.

Figure 14. Percent Muscle Activity (\%MA) values for the five Latisimmus Dorsi musculotendon units from the 10-pound lifting task modeled with the MMS recruitment strategy during the dynamic portion of the task. 36

Figure 15. Percent MVC (\%MVC) values for the Latissimus Dorsi muscle from the 10-pound lifting task during the dynamic portion of the task. .36

Figure 16. Correlation values stratified by weight and recruitment strategy, including the mean correlation for each weight condition and associated standard deviation bars, for the dynamic analysis. .37

Figure 17. Correlation values stratified by muscle and weight for the dynamic analysis.........................38

Figure 18. Mean correlation values and mean RMSE values found for each recruitment strategy for the static analysis. 39

Figure 19. Correlation values stratified by weight and recruitment strategy for the static analysis. .......... 40

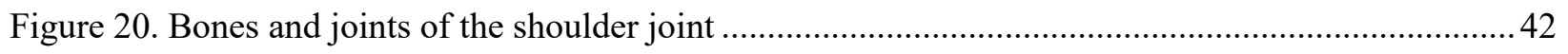

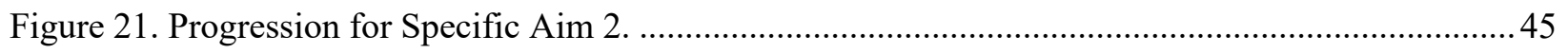

Figure 22. Custom-built wooden structure to support hanging weight............................................... 47

Figure 23. Top) Actual foamboard grid used for the experimental tasks. Bottom) Digital mock-up of foamboard grid layout for easier visualization. 
Figure 24. Two-dimensional angular deviations. In this figure, $\alpha, \beta$, and $\gamma$ are the angles that were normalized by $180^{\circ}$ using Equations 7, 8, and 9.

Figure 25. Stability modifier values determined from Lippitt et al. translational/compressive load stability results interpolated with a Piecewise Cubic Hermite Interpolating Polynomial function. 60

Figure 26. Representation of how the distances between the sternal notch and load are measured along each axis.

Figure 27. Predicted Strain with the load 20 inches above the sternal notch..........................................6 68

Figure 28. Predicted Strain with the load 15 inches above the sternal notch...........................................69

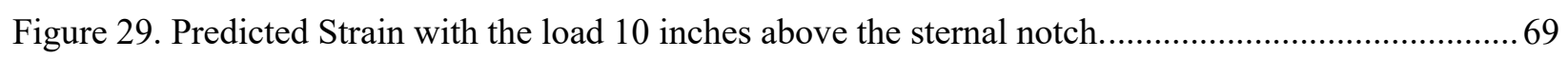

Figure 30. Predicted Strain with the load 5 inches above the sternal notch............................................. 70

Figure 31. Predicted Strain with the load horizontally aligned with the sternal notch. ............................. 70

Figure 32. Predicted Strain with the load 5 inches below the sternal notch. ............................................ 71

Figure 33. Predicted Strain with the load 10 inches below the sternal notch. .......................................... 71

Figure 34. Predicted Strain with the load 15 inches below the sternal notch. .......................................... 72

Figure 35. Predicted Strain with the load 20 inches below the sternal notch. ......................................... 72

Figure 36. a) One of two matching tripods used as part of the adjustable shelf system. b) One of two 4" by 4" wooden platforms with pronged T-nut embedded. c) Wooden platform attached to tripod. d) Height range markings to allow easy height adjustability of tripods..................................... 77

Figure 37. Ten-pound weight with D-handle used as the load during lifting tasks. Retroreflective marker at

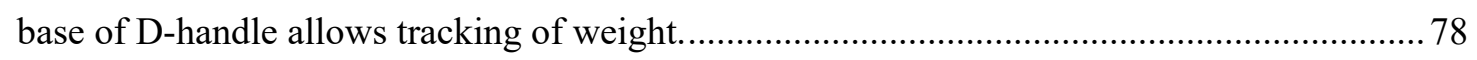

Figure 38. Plumb bob that was hung from tripod to precisely align the tripods to the proper location....... 80

Figure 39. Example progression of an experimental task, showing the participant starting from a neutral position, grasping the weight, placing it onto the destination tripod, and returning to neutral

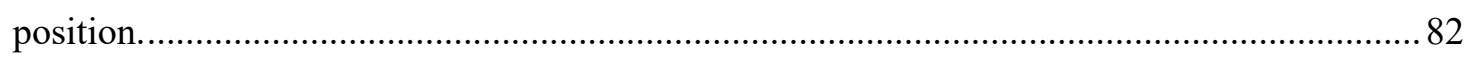

Figure 40. Predicted strain (solid line) and composite index (dashed line) for Low 1 to High 1 task........ 87

Figure 41. Predicted strain (solid line) and composite index (dashed line) for Low 1 to Medium 1 task. . 88

Figure 42. Predicted strain (solid line) and composite index (dashed line) for High 1 to High 2 task....... 88

Figure 43. Predicted strain (solid line) and composite index (dashed line) for High 2 to High 1 task....... 89

Figure 44. Glenohumeral force components for Participant 2 performing the High 2 to High 1 and Low 1 to Medium 1 tasks. 90

Figure 45. Musculotendon activations for Participant 2 performing the High 2 to High 1 and Low 1 to

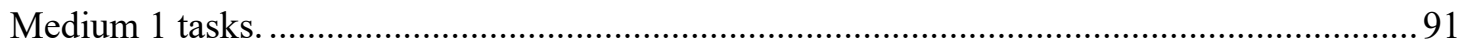

Figure 46. Trajectory and strain plots for the High 1 to High 2 experimental task.. .............................. 92

Figure 47. Trajectory and strain plots for the Low 1 to Medium 1 experimental task..............................93 
Figure 48. Trajectory and strain plots for the High 1 to Low 1 experimental task............................... 94

Figure 49. Trajectory and strain plots for the Medium 1 to High 1 experimental task............................ 95 


\section{List of Tables}

Table 1. List of 32 retro-reflective marke rs in the marker set and their location on the body. 18

Table 2. Muscles that were included in the analysis, including action of the muscle, the location of the muscle, and the placement of the electromyography sensor.

Table 3. Muscles that were studies in this research, their representative musculotendon units from the model, and each musculotendon unit's maximum strength and weighting. .28

Table 4. Overall mean correlation and RMSE values for each recruitment strategy for dynamic analysis.

Table 5. Correlation values stratified by muscle and recruitment strategy for the dynamic analysis......... 35

Table 6. Overall mean correlation values and mean RMSE values for each recruitment strategy for the static

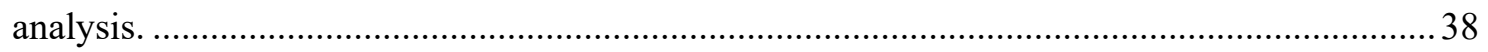

Table 7. Heights and weights of each participant in Specific Aim 2 .................................................. 46

Table 8. Per-participant breakdown of number of collected trials, losses during motion capture, losses during modeling, and successful trials used in final analysis.

Table 9. Correlations coefficients and p-values resulting from correlation analysis between each of the potential components and the EMG Summation and perceived exertion rating. .56

Table 10. Results of correlation analysis between the perceived exertion values and each of the 12 potential composite index equations.

Table 11. Results of correlation analysis between the EMG summation values and each of the 12 potential composite index equations.

Table 12. Summary results from the Best Sets analysis, including the best single predictor and the number of predictors in the Best Set of each subset of the 11 sets of predictors.

Table 13. Correlations between the predicted strain values data using each regression equations and the composite index values, calculated from the participant data, and perceived exertion rating; RMSE calculated between composite index values and predicted strain values for each regression equation.

Table 14. Distances Forward (F), Right (R) or Left (L) and Up (U) or Down (D) from the sternal notch to the load.

Table 15. Mean (of two repetitions) correlation coefficients and root mean square error values between predicted strain values and composite index values for each participant and task.

Table 16. Predicted strain values at the Origin (O) and Destination (D) of the lifting task and the max (M) predicted strain hacross the entire lifting task. Cells highlighted in yellow, orange, and red are 
trials where the highest strain value did not occur at either the origin or destination in 1-2,3-4, or 5 and more trials, respectively.

Table 17. Breakdown for each participant of number of different trials, where the strain was highest at some point other than the origin or destination, and the mean differences across all trials and only the different trials. .86 


\section{INTRODUCTION}

\subsection{Background}

\subsubsection{Shoulder Musculoskeletal Disorder Prevalence and Cost}

Workplace injuries and musculoskeletal disorders (MSDs) commonly affect the shoulders, resulting in considerable direct and indirect costs. According to data from the U.S. Bureau of Labor Statistics (2017), shoulder injuries represented 14.9\% of all work-related musculoskeletal disorders in 2016. Despite being less common than musculoskeletal disorders of the low back and knee, injuries to the shoulder tend to be the most severe, requiring a median of 25 days away from work compared to 7 and 15 days for the low back and knees, respectively. Several analyses have been performed to determine the prevalence, costs, characteristics, and outcomes of Work-related MSDs in different states.

An analysis of Washington State Worker's Compensation data from 1997-2005 revealed shoulder claims as having the highest per claim cost, with an average cost $\$ 16,092$ per claim and a median cost of $\$ 1,111$ per claim over 46,479 claims, resulting in a total claim cost of $\$ 645$ million (Silverstein and Adams, 2007). Similar analyses were performed with data from 2002-2010 (Anderson et al., 2015) and 2006-2015 (Howard and Adams, 2018) revealing an average per claim cost of $\$ 60,298$ over 29,046 claims and $\$ 65,053$ over 31,366 claims, respectively. Davis et al. (2014) performed an analysis of Ohio Worker's Compensation data from 1999-2004 that also stratified the data by age category to elicit any age-related trends in claim characteristics. The effect of age on mean per claim cost across all claims trended upward from the $16-24$ year age range $(\$ 1,722$ /claim) up to $55-64$ year age range $(\$ 6,875 /$ claim $)$, before dropping slightly in the $65+$ year age range $(\$ 5,715)$. This trend maintained when only considering shoulder claims, with mean claim costs rising from the 16-24 year age range ( $\$ 1,524 /$ claim) up to the 55-64 year age range $(\$ 10,130)$, before dropping slightly for the $65+$ year old cohort $(\$ 9,564)$. 


\subsubsection{Ergonomic Analysis Methods of the Shoulder}

Due to the high medical costs, loss of productivity, and reduced quality of life due to shoulder MSDs, there is a drastic need for workplace job analysis to determine the risk for shoulder MSDs. Currently, very few methods exist that consider the shoulder. Rapid Upper Limb Assessment (RULA) is a qualitative task analysis tool where posture of the upper body, muscle use (repetitive vs. static load), and force/load are used to generate a grand score between 1 and 7 that is broken down into 3 risk categories (McAtamney

et al. 1993). Since the posture determinations are approximate ranges and the other scores are based on easy to determine criteria (e.g. "Add 1 if trunk is twisting"), RULA requires no special equipment and only a rudimentary knowledge of kinesiology making it very easy to apply. However, the grand score doesn't provide any information specific to the shoulder and only advises whether the task is acceptable or how urgently it needs to be addressed.

The Rapid Entire Body Assessment (REBA) tool functions similarly to RULA, but uses full body posture, force/load, and load coupling as criteria to generate a score between 1 and 15, divided into 5 risk categories (Hignett and McAtamney 2000). While REBA expands on RULA by including the lower body in the assessment, the method suffers the same drawbacks as and is no more useful than RULA for shoulder assessment.

The Liberty Mutual Manual Materials Handling Tables, also known as the Snook Tables, are a set of tables that can be referenced to estimate a maximum acceptable load/force for lifting/lowering, pushing/pulling, and carrying tasks based on task characteristics such as distance, repetitions, and load origin and destination (Snook \& Ciriello, 1991). The Snook Tables are most useful when the load can be changed, since they advise the appropriate load based on task characteristics. If changing the load is not feasible, the Snook tables are less useful than other methods. Additionally, the Snook tables only cover a limited number of values for the distances. For instance, the tables for lifting tasks only account for hand distances of 7, 10, and 15 inches in front of the body. 
Rodger's Muscle Fatigue Analysis is a full-body fatigue analysis tool that was developed around the hypothesis that fatigued muscles are more prone to inflammation and injury (Rodgers, 1991). The effort, duration, and frequency of motion of each of the seven major body regions (neck, shoulders, back, arms/elbow, wrist/hand/fingers, legs/knees, and ankle/feet/toes) during a task are rated from 1 to 4 based on given criteria. The criteria for the frequency and duration are the same for all body regions, but the effort criteria are different for each region. The ratings are strung together to form a 3-digit code that corresponds to a level of risk (low, moderate, high, very high) provided in a table. While this tool is simple to use and can be used to evaluate an individual body region, its analysis for each body region is very basic.

\subsubsection{Risk Factors for Shoulder Musculoskeletal Disorders}

Several different risk factors for shoulder musculoskeletal disorders have been well established in prior literature, including heavy physical load, repetitive motions, working with the arm above the shoulder, awkward postures, vibration, and prolonged performance of the same task. A complete literature review of these risk factors can be found in Appendix A. Each of these risk factors are thought to have different pathological results due to differing pathways. Repetitive motions at the shoulder has been shown to increase intramuscular pressure in the rotator cuff muscles, restricting bloodflow to the tendons and resulting in inflammation (Frost et al. 2002). Additionally, working with the arm above the shoulder also causes an increase in intramuscular pressure of the rotator cuff muscles, due to their continuous use stabilizing the Glenohumeral joint. Working with the arms above the shoulder can also cause shoulder impingement, squeezing the subacromial tendon between the humeral head and the underside of the acromion (Winkel and Westgaard, 1992). Heavy physical loads can cause dislocation, increased tendon tension, and impair circulation. It is common for many of these risk factors to exist concurrently, and these risk factors often have a strong relationship with fatigue.

Fatigue and intramuscular pressure go hand in hand. In fact, Korner et al. (1984) found accumulated muscle fatigue only occurred at intramuscular pressures over $20 \mathrm{mmHg}$ in the Biceps Brachii muscle. Crenshaw et al. (1997) explored the relationship between intramuscular pressure and fatigue 
indicators during low- and high- level isometric contractions ( $25 \%$ and $70 \%$ of $\mathrm{MVC}$, respectively), finding that as an isometric exertion continues toward fatigue, intramuscular pressure and fatigue (evidenced by decreased mean frequency) have a strong linear relationship, especially during low-level isometric contractions. During the high-level isometric contractions, the correlation was not as strong, but this was, at least in part, due to intramuscular pressure peaking near the same value $(55.8 \pm 27 \mathrm{mmHg})$ as those for the low-level exertions, however the peak intramuscular pressure developed faster during the high-level exertions. Previous research has shown that intramuscular pressure exceeding 30 to $50 \mathrm{mmHg}$ can reduce local blood flow (Jarvholm et al. 1988).

\subsection{Specific Aims}

The primary objective of this research was to develop and validate the use of a workplace analysis tool to determine the strain on the shoulder during lifting tasks (e.g. moving an object from a shelf to a conveyor belt) based on the origin and destination of the load. The following three aims were undertaken:

\section{Specific Aim \#1: Muscle recruitment strategy determination for modeling tasks}

Human participants performed static and dynamic lifting tasks, which were modeled in a biomechanical modeling software to compute various physiological outputs, including predicted muscle activity and resultant forces at the Glenohumeral joint. The software includes multiple muscle recruitment strategies (i.e. algorithms) that can be selected, which determine how the work is distributed between the muscles for a certain action. In the human body, and consequently in the model, the force at the Glenohumeral joint is a result of the muscles pulling, therefore, as muscle activity accuracy increases in the model, the ability to accurately predict the forces at the Gleohumeral joint also increase. Predicted muscle activity data from 13 muscles in the model when using six different muscle recruitment strategies was compared with electromyography data from the corresponding muscles using correlation and RMSE analysis to determine which muscle recruitment strategy provides the most accurate representation of the 
muscle activity, which should have provided better prediction of forces on the Glenohumeral joint, for static and dynamic tasks.

\section{Specific Aim \#2: Composite index and predictive equation development}

Human participants performed static lifting exertions at specific points throughout the working range of the right arm, immediately providing a rating of their perceived exertion on a Borg CR-10 scale (1-10 rating based on strength of exertion) for each exertion. These tasks were modeled using the muscle recruitment strategy that was chosen in Specific Aim 1 for static exertions. A composite index that depicts strain on the shoulder joint was developed based on model outputs (Glenohumeral reaction forces), physiological principals and prior research that correlates well with the exertion rating and collected electromyography data. Regression analysis was used to develop a predictive equation that relates the location of the load with respect to the sternal notch with the composite index values. This data was used to develop heatmaps that visually depict the strain throughout the working range.

\section{Specific Aim \#3: Validation of predictive equation as task analysis tool}

To test the theory that the strain on the shoulder during an unobstructed lifting task is highest at either the origin or destination and, therefore, validate the use of the origin and destination of the load to analyze the shoulder strain induced by a lifting task, a location of high, medium, and low strain from within the working range of the right arm were selected with guidance from the heatmaps. Human participants performed several tasks lifting a 10-pound object from and to combinations of high, medium, and low strain locations. Each task was modeled using the muscle recruitment strategy that was chosen in Specific Aim 1 for dynamic exertions. Correlations between calculated composite index values and predicted strain values further supported the use of the predictive equation for estimation of shoulder strain. Predicted strain values were calculated throughout each lifting task to determine if the peak strain occurs at one of the endpoints of the lift. 


\subsection{Significance}

Due to the high direct and indirect costs of shoulder MSDs, there is a great need for a workplace task analysis tool with a focus on the shoulder. Currently, no such tool exists. Therefore, this research set out to create a much-needed shoulder analysis tool. Participants performed static lifting exertions throughout the working range of the right arm. Each task was modeled and the model outputs were used to develop a Composite Index to quantify strain on the shoulder. A predictive equation was developed to estimate strain (i.e. composite index) based on the location of the load relative to the top of the sternum. Finally, the theory that the highest strain during an unobstructed lifting task exists at either the origin or the destination will be tested, which, if supported, will validate using this tool at the origin and destination of a task to make an assessment about the entire task. Expected outcomes of this research were as follows:

1) Collected electromyography data was compared to model outputs to determine the best muscle recruitment strategy for analysis of static and dynamic tasks. This contributes to the field by providing further evidence of the accuracy of the AnyBody musculoskeletal modeling system and determining the most appropriate muscle recruitment strategies in the software to use for static and dynamic tasks of the shoulder, allowing use of the model with more confidence without extensive validation.

2) A predictive equation and heatmaps to determine the strain on the shoulder when lifting a load based on the location of the load were developed. This provides a framework for a full analysis tool to estimate the risk of static force exertion tasks based on the location of the load and other task characteristics

3) While results showed that the strain is not always highest at the origin or destination of the lift, it revealed that this was due to an arced lifting trajectory and the differences were, in most cases, minimal. Therefore, once a full analysis tool has been developed, a pathway toward an origin/destination analysis tool was provided. 


\section{SPECIFIC AIM \#1: MUSCLE RECRUITMENT STRATEGY DETERMINATION FOR MODEELING TASKS}

\subsection{Background}

Throughout this research, AnyBody Modeling System 6.0 (AnyBody Technologies, Aalborg, DK) was used to model the experimental tasks. The software uses an object-oriented scripting language to create musculoskeletal models with bones, joints, and muscle-tendon units and is capable of accounting for complex physiological properties during the analysis, including muscle length, contraction velocity, fiber length, pennation angle, tendon elasticity, and stiffness of passive tissues (AnyBody Tutorial, Chapter 5), making it much more robust and flexible than simple linked-segment models. Experimental data can be used as input into AnyBody models in several ways. Motion of the model can be driven by specifying frame-by-frame joint angles in a text file, manually entering them into the model, providing an equation with a time variable, or with motion-capture data. External loads can be specified using force sensor data, force plate data, or can be manually input into the model.

A full-body motion-capture driven gait analysis model (MocapModel-FullBody) from the AnyScript repository was modified for use in this research by removing the leg and foot segments and related markers from the model since the experimental tasks are limited to upper body motion (Figure 2). By making this modification and requiring the participant to perform the tasks in a specified posture, data processing and participant preparation time will be saved with no effect on the quality of the data that will be used for this research.

Marker locations in the model are defined by their relative three-dimensional position on a segment where the three-dimensional midpoint of the segment is represented by $\{0,0,0\}$ and the endpoints along all three axes are represented by -1 and 1 . When marker data is input into the model, the lengths and orientations of the segments are determined using optimization methods to minimize the error between the modeled marker coordinates and the experimentally-derived marker data. Once the motion capture data is 
used to derive the model kinematics, inverse dynamics calculations are used to determine the muscle recruitment required to perform the motion. Subsequently, many physiological properties during the activity are determined, including muscle and tendon length, muscle activity, and internal forces at the joints.

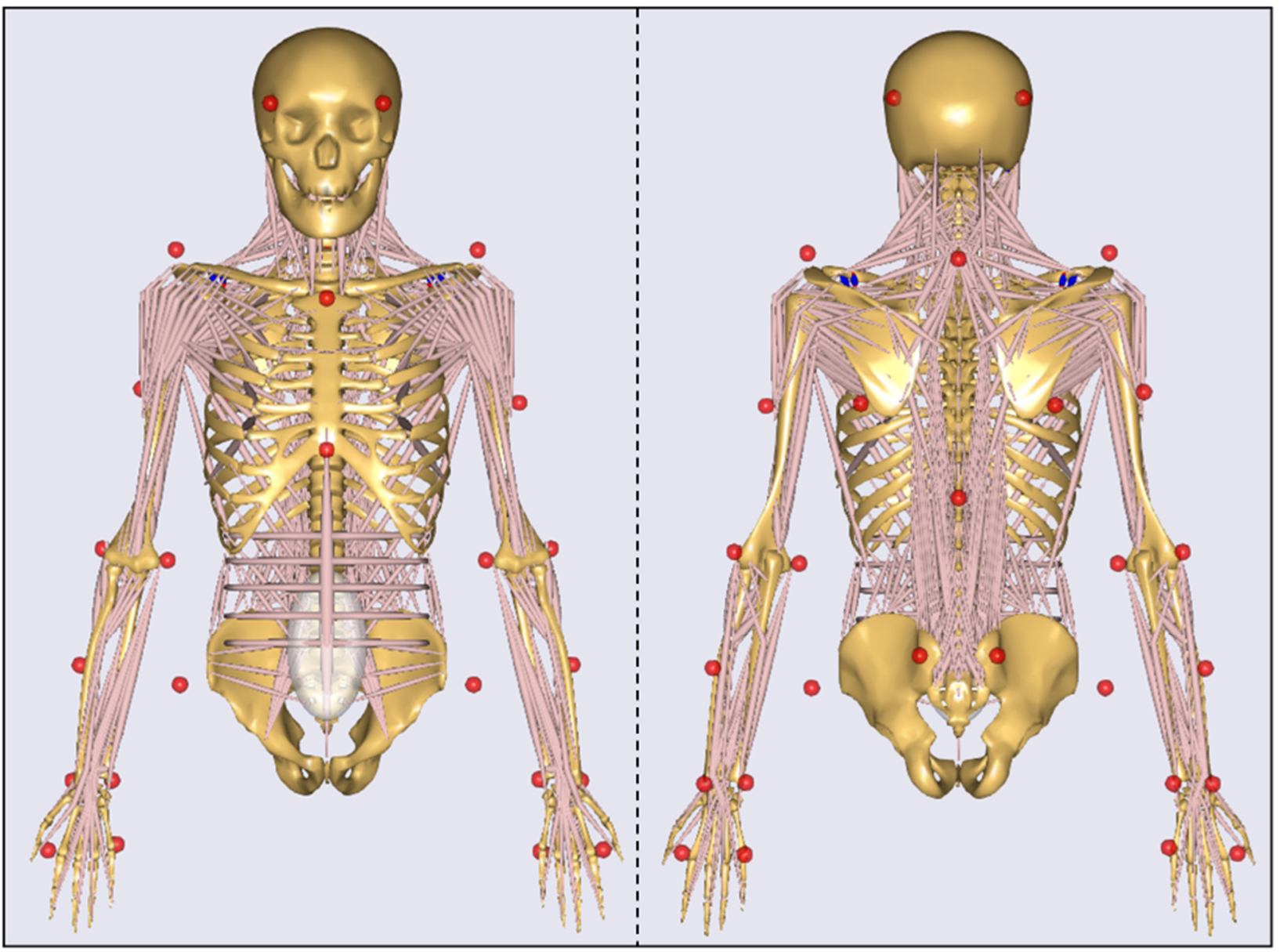

Figure 1. Model View of the modified MoCap-FullBody model that was used throughout this research.

For most accurate determination of muscle recruitment, the most appropriate muscle recruitment strategy must be selected. AnyBody software offers linear, quadratic, polynomial, $\min / \max$, and composite muscle recruitment strategies that rely on optimization. Linear, quadratic, and polynomial muscle recruitment minimize Equation 1, where the value for $\mathrm{p}$ determines muscle recruitment pattern. Composite 
muscle recruitment uses a similar form, however with minimization of a quadratic term and an additional, weighted, linear term (Equation 2). Finally, $\min / \max$ muscle recruitment minimizes the maximal muscle activity using an internal algorithm represented in Equation 3.

$G=\sum_{i}\left(\frac{f_{i}}{N_{i}}\right)^{p}, \mathrm{p}=1,2, \ldots \mathrm{n}$

(Equation 1)

where:

$$
\begin{aligned}
& p=1 \text { for linear, } \\
& p=2 \text { for quadratic, and } \\
& p=3 \text { or more for polynomial. }
\end{aligned}
$$

$G=\sum_{i}\left(\frac{f_{i}}{N_{i}}\right)^{2}+x\left(\frac{f_{i}}{N_{i}}\right)$

(Equation 2)

where $\mathrm{x}=(0,1)$.

$G=\max \left(\frac{f_{i}}{N_{i}}\right)$

(Equation 3)

Linear muscle recruitment will recruit the fewest muscles possible to perform the action, beginning with the strongest muscle. Linear muscle recruitment is not physiologically realistic, however. Quadratic muscle recruitment is much more physiologically appropriate, allowing synergism between muscles by penalizing large terms. Polynomial muscle recruitment with power $\mathrm{p}=3$ shows increased muscle synergism, with muscle synergism further increasing as the power of the equation increases. As the power of the polynomial muscle recruitment algorithm nears infinity, the algorithm becomes less numerically attractive and requires upper bound constraints to be specified. The $\min / \max$ recruitment strategy, which converges with the polynomial muscle recruitment algorithm for large values of $p$, is more numerically attractive due to reduction to one equation with one unknown and implicitly fulfills the upper bounds 
constraints for sub-maximal loads due to maximizing muscle synergism (Rasmussen et al. 2001). However, maximizing muscle synergism results in exploitation of muscles with small moment arms that may not be realistic (Forster, 2003).

Research has been performed using each of these recruitment methods but validation was usually limited to comparing modeled muscle activity to experimental EMG data for only the muscle recruitment pattern used (Erdemir et al., 2007). For instance, Zee et al. (2007) developed a musculoskeletal model of the skull and mandible in order to research mandibular distraction osteogenesis by comparing reaction forces at the left and right temporomandibular joints. They validated their model by comparing electromyography data collected with fine-wire and surface electrodes while participants performed 5 tasks with muscle activity predicted by the modeled tasks, resulting in an overall correlation of 0.580 and a Mean Absolute Error of 0.109 . They found these values to be reasonable, but noted that the muscle recruitment strategy that was used, min/max criterion, may not be valid for all cases. Additionally, they noted that $\min /$ max recruitment strategy relies on the assumption that the muscles are recruited in an optimal way, suggesting that $\min / \max$ recruitment strategy may be optimal when performing a familiar movement, but not when performing an unfamiliar or unskilled activity. On the other hand, Han et al. (2012) were interested in improving the accuracy of a general spinal model by including short segmental muscles, lumbar ligaments, and disc stiffness and subsequently validated the accuracy of the model compared to the base model by comparing their modeled data to data from prior research. Several activities of daily living were modeled using the base model and the improved model and the joint reaction forces were compared against in vivo implant forces and intradiscal pressures from other research. The analysis found better agreement to the research data with the improved model than the base model.

No consensus exists on which muscle recruitment strategy is most accurate. It's possible that the most appropriate muscle recruitment strategy will depend on the type of motion involved (dynamic or static). 


\subsection{Objectives and Hypotheses}

Objective 1: Determine the most appropriate muscle recruitment strategy for use during the modeling of dynamic tasks and static tasks of the shoulder in AnyBody musculoskeletal modeling system. The forces experienced at the Glenohumeral joint are primarily a result of the activation of the muscles of the shoulder. Each muscle has a different attachment point and a different line of action at the shoulder. Accurate determination of muscle activation was necessary to estimate forces at the Glenohumeral joint with any accuracy.

Null Hypothesis: The recruitment strategies will not affect the muscle activity outputs in the model during static and dynamic exertions.

Alt. Hypothesis: The recruitment strategies will affect the muscle activity outputs in the model during static and dynamic exertions.

\subsection{Approach}

Participants were recruited to perform a lifting task with three different weights, each consisting of a dynamic and static portion. While performing the experimental tasks, electromyography data and motion capture data were collected. The tasks were modeled with AnyBody Modeling System using the following available muscle recruitment strategies: Quadratic (Quad), 3rd-order Polynomial (Poly3), 4th-order Polynomial (Poly4), 5th order Polynomial (Poly5), Min/Max (MMS), and Composite (Comp). Experimentally collected electromyography data was compared to model-derived predicted muscle activity from each recruitment strategy by comparison of correlation and root mean square error (RMSE) values to determine which muscle recruitment strategy is most appropriate.

\subsection{Participants}

A convenience sample five healthy male participants with mean $( \pm \mathrm{sd})$ age, height, and weight of 25.6 ( \pm 3.8$)$, years, $1.76( \pm 0.03)$ meters, and $70.3( \pm 8.5) \mathrm{kg}$, respectively, were recruited for this study. For 
inclusion in the study, potential participants had to be male, aged 18-40 years old, and have no musculoskeletal disorders that would affect their ability to perform the tasks or that could otherwise affect the results. Participants all confirmed to being right-hand dominant. Male participants were used because several of the electromyography sensors must be placed on the chest, and the location that the chest sensors were placed is much more private on females than males. Additionally, breast tissue could interfere with the muscle activity recordings.

\subsection{Equipment}

In addition to the AnyBody modeling system software, the following equipment were used during this stage of the research.

2.5.1 Data Acquisition Hardware: Electromyography and motion capture systems were used in this research. The systems are described in detail in the following subsections. Additionally, a schematic of how the systems were physically connected is provided below (Figure 3).

2.5.1.1 Optical Motion Capture System (MX Series, Vicon Motion Systems, Oxford, UK): An eight-camera, infrared, optical motion capture system and accompanying software was used to track the three-dimensional location of $12 \mathrm{~mm}$ retro-reflective markers attached to the participant. The system consisted of an MXControl unit, an MXUltranet unit, an Analog to Digital Interface unit, and $8 \mathrm{MX}+$ Cameras (Figure 4). The eight infrared cameras were connected to the MXUltranet unit, which relays the camera data to the MXControl Unit. The Analog to Digital Interface Unit connected to the MXControl unit, as well, to aggregate and relay data from other analog devices, which in this research was an electromyography system. The MXControl unit connected to the PC and supplied the data to the Vicon Nexus 1.8.1 software. In this research, the motion capture data was collected at a rate of $100 \mathrm{~Hz}$. 
2.5.1.2 Electromyography System (Bagnoli-16, Delsys Inc., Natick, MA, USA): A 16-channel desktop electromyography system was used to measure electrical activity of the muscles. The system consisted of the Bagnoli Desktop unit, two portable EMG signal receivers, and surface EMG sensors (Figure 5). The

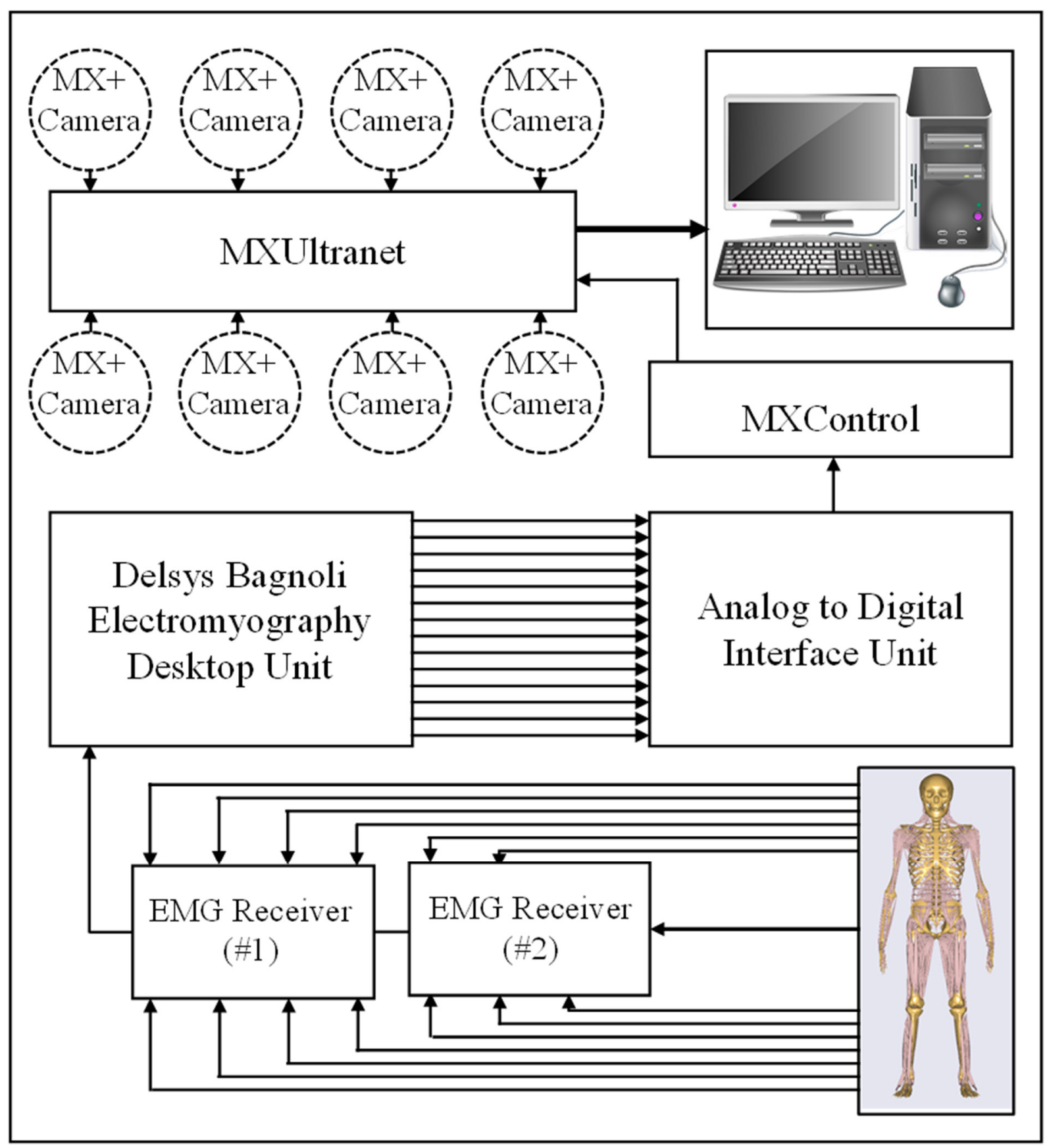

Figure 2. Schematic of the physical connectivity of the data acquisition hardware. 
Bagnoli Desktop unit connected to the Vicon Analog to Digital Interface unit, which allowed the electromyography data to be collected within the Vicon Nexus software. The portable EMG receivers attached to the participant with belt clips. The EMG receiver with channels 1-8 connected to the Bagnoli Desktop unit. The other EMG receiver connects to the first EMG receiver. Single-Differential Surface Electromyography sensors (Bagnoli DE-2.1) with parallel bar electrodes (1cm Inter-electrode distance, 92dB CMMR, and $>100 \mathrm{M} \Omega$ Input Impedence) attach to the portable EMG receivers which relayed the signal to the Bagnoli Desktop. EMG Output was synchronized and recorded by the Vicon Nexus 1.8.1 software at a rate of $1000 \mathrm{~Hz}$.

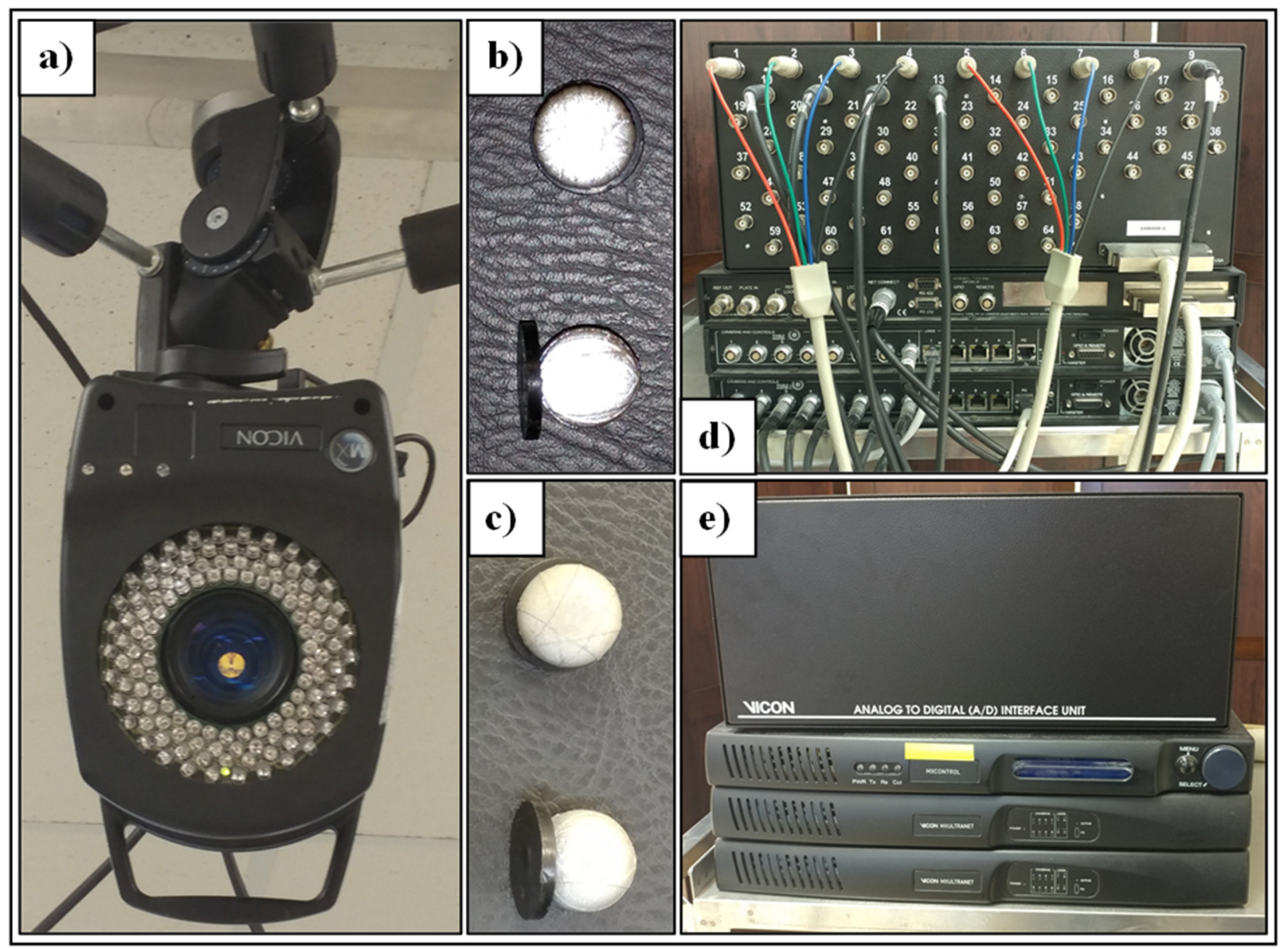

Figure 3. Images of the Vicon system hardware. (a) Vicon MX+ Series infrared motion capture camera. (b \& c) $12 \mathrm{~mm}$ retro-reflective markers, with image taken with and without camera flash. (d \& e) Front 
and rear of Analog to Digital Interface Unit, MXControl, and an inactive and active MXUltranet unit, from top to bottom of stack.

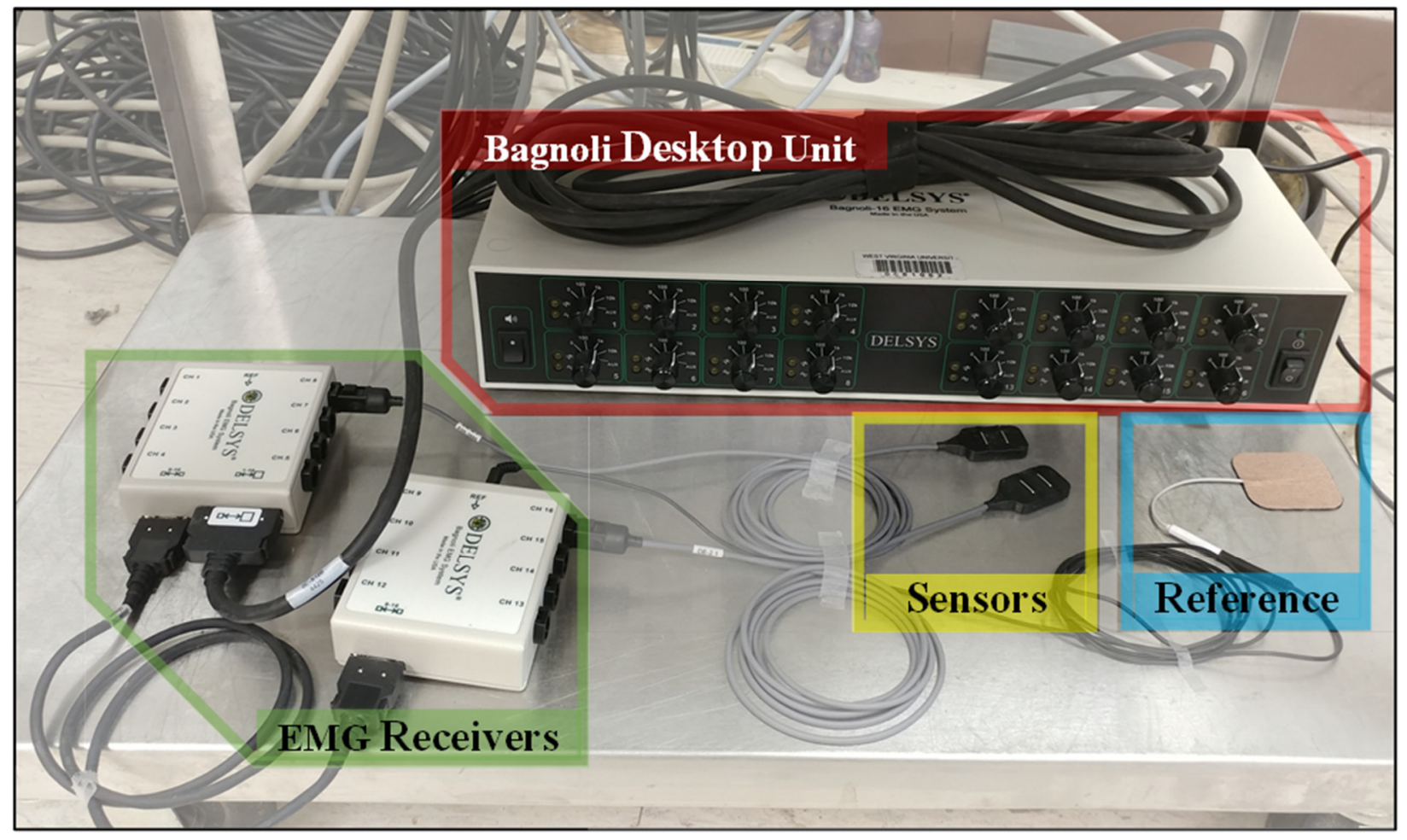

Figure 4. Components of the Delsys Bagnoli electromyography system: Bagnoli desktop unit, two portable EMG receivers, two electromyography sensors and a reference sensor.

2.5.2 Rehabilitation Machine (Humac Norm, CSMi Solutions, Stoughton, MA, USA): A physical therapy rehabilitation machine was used to maintain the posture and provide the resistance required for the maximum voluntary contraction exertions (Figure 5). The machine consists of a seat and dynamometer, each with several adjustments to their position, as well as several accessories that attach to the dynamometer. The machine is designed to isolate the posture and control the movement of rehabilitation exercises, which provides us the flexibility we need.

2.5.3 Weights: Flat weights weighing 2.5 pounds each were bolted to a D-handle to create the weights needed for this portion of the research. One, two, and four weights were bolted to the D-handle to create, respectively, 2.5 pound, 5 pound, and 10 pound weights that were easy to grip (Figure 6). 


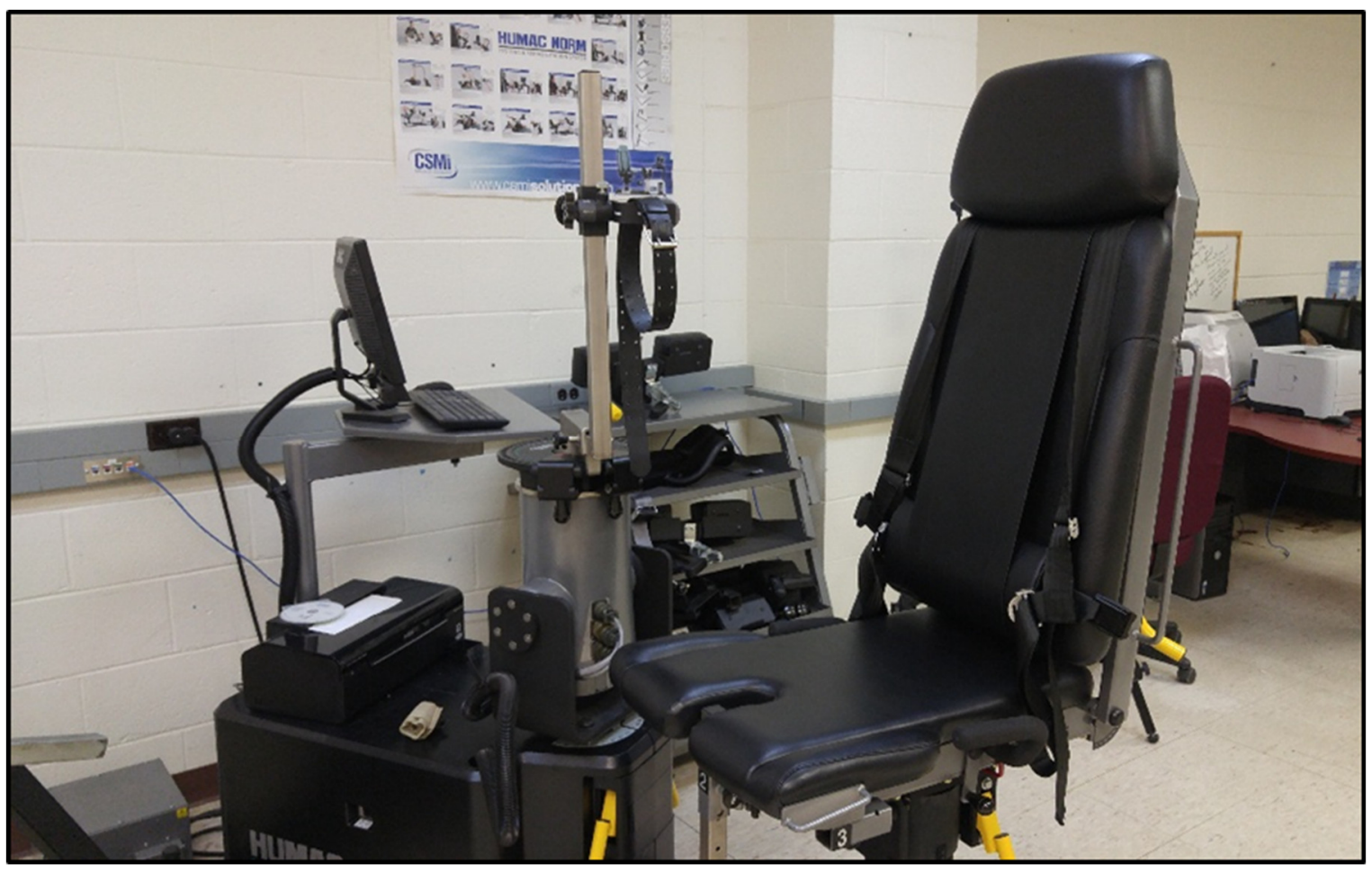

Figure 5. Rehabilitation machine used during maximum voluntary exertions to control motion and provide resistance where needed.

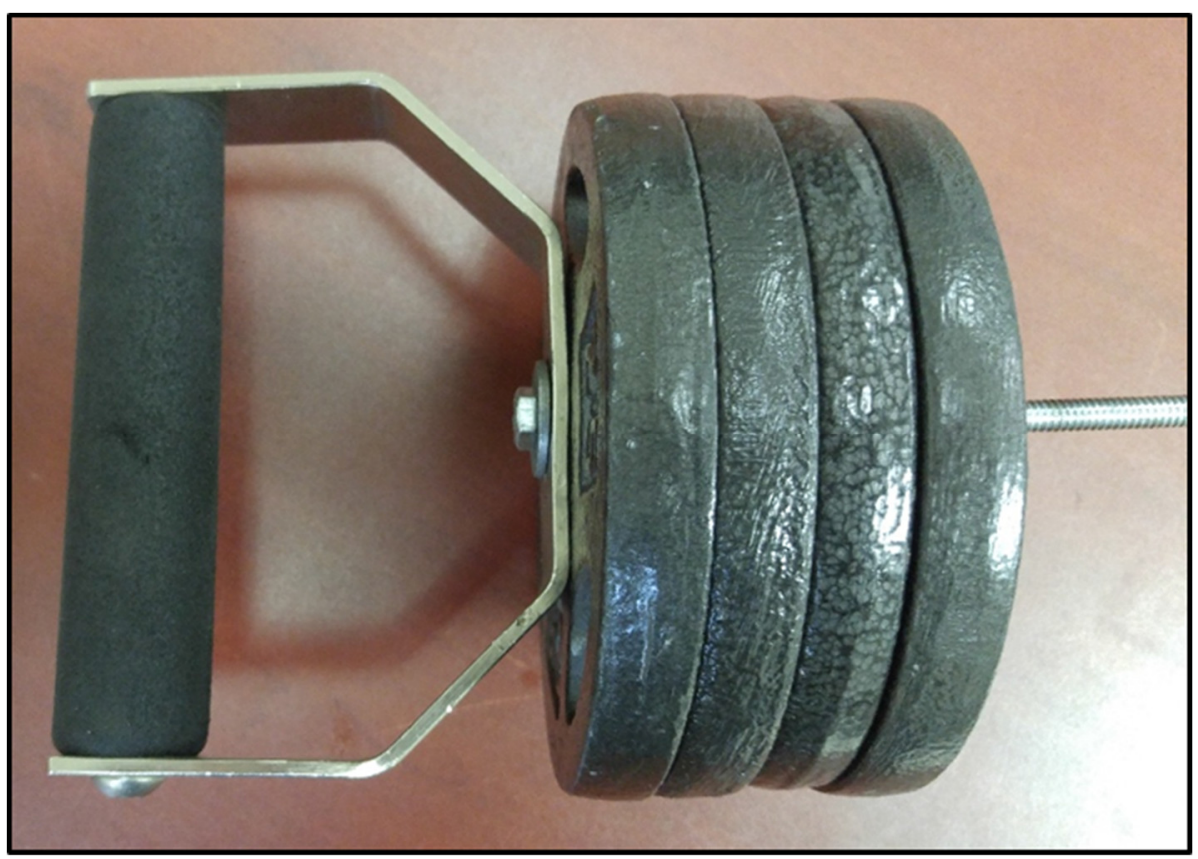

Figure 6. D-handle bolted to four flat weights weighing 2.5 pounds each, resulting in a 10-pound weight. 


\section{$\underline{\text { 2.6 Experimental Design }}$}

For analysis of the dynamic portion of the tasks, the effect of recruitment strategy, muscle, and weight on the Pearson correlation and RMSE values was explored. For the static portion of the tasks, the effect of recruitment strategy and weight on the Spearman correlation and RMSE values was explored. The most accurate muscle recruitment strategy for dynamic and static exertions, based on a high correlation with electromyography data and a low RMSE, will be used in the remainder of this research when tasks are modeled.

\section{$\underline{\text { 2.7 Participant Consent and Preparation }}$}

Upon arrival, participants were introduced to the experimental setup and consent was obtained with a consent form approved by the local institutional review board (Appendix B). The participant was screened for cardiorespiratory fitness with the Physical Activity Readiness Questionnaire (PAR-Q) and accepted for participation if all answers were "Yes", indicating no cardiorespiratory risks (Appendix C). Participants removed their shirt. A marker set including thirty-two $12 \mathrm{~mm}$ retro-reflective markers was used in this research. Twenty-four of the markers were affixed to the skin of the participant at specific anatomical landmarks with double-sided tape. Four markers were attached to the shorts with double-sided tape. The final four markers are on a headband that was worn by the participant (Figure 7). Locations of each marker and their identifying label can be found in Table 1.

Thirteen electromyography sensors were placed on the following muscles: Anterior, Middle, and Posterior Deltoid, Clavicular, Sternal, and Abdominal branches of the Pectoralis Major, the Lateral and Medial branches of the Biceps Brachii, Tricep, Latissiumus Dorsi, Infraspinatus, Supraspinatus, and Teres Major (Figure 8) after shaving the sensor location and cleaning it with isopropyl alcohol. These muscles were selected because they are all responsible for movement and/or stability of the shoulder joint. Locations for sensor placement, along with information on the action and location of each muscle, are described in 


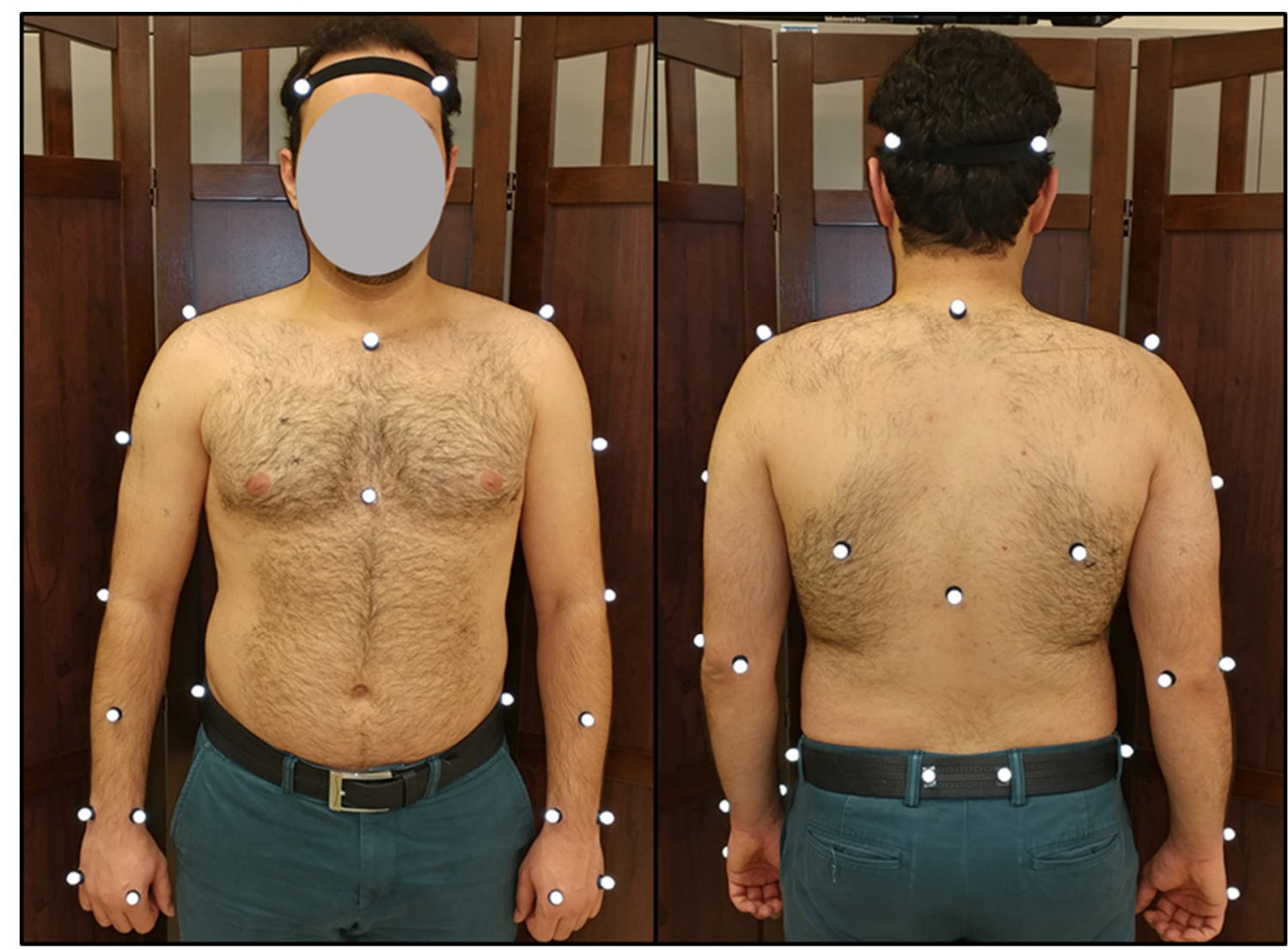

Figure 7. Set of 32 retro-reflective markers in the marker set attached to a participant.

Table 1. List of 32 retro-reflective marke rs in the marker set and their location on the body.

\begin{tabular}{|c|c|c|c|}
\hline \multicolumn{2}{|r|}{ Head } & \multicolumn{2}{|r|}{ Right / Left Arm } \\
\hline RFHD & Right side of forehead & RUPA/LUPA & Midpoint of upper arm on lateral side \\
\hline RBHD & Right side of back of head & RLELB/LLELB & Lateral epicondyle of humerus at elbow \\
\hline LFHD & Left side of forehead & RMELB/LMELB & Medial epicondyle of humerus at elbow \\
\hline LBHD & Left side of back of head & RFRM/LFRM & Midpoint of forearm \\
\hline \multicolumn{2}{|r|}{ Trunk } & RWRA/LWRA & Radial styloid at wrist \\
\hline $\mathrm{C7}$ & Spinous process of $\mathrm{C} 7$ vertebrae & RWRB/LWRB & Ulhar styloid at wrist \\
\hline CLAV & Sternal notch at top of sternum & RFIN/LFIN & 5th MCP joint of the hand \\
\hline STRN & Xiphoid process at base of sternum & RTHB/LTHB & 2nd MCP joint of the hand \\
\hline T10 & Spinous process of $T 10$ vertebrae & \multicolumn{2}{|r|}{ Pelvis } \\
\hline RSHO & Right acromion process of shoulder & RASI & Right anterior iliac spine \\
\hline LSHO & Left acromion process of shoulder & RPSI & Right posterior iliac spine \\
\hline RBAK & Inferior angle of right scapula & LASI & Left anterior iliac spine \\
\hline LBAK & Inferior angle of left scapula & LPSI & Left posterior iliac spine \\
\hline
\end{tabular}


Table 2. Additionally, a sensor to provide a reference signal was placed on the forehead above the left eye, where there is no musculature.

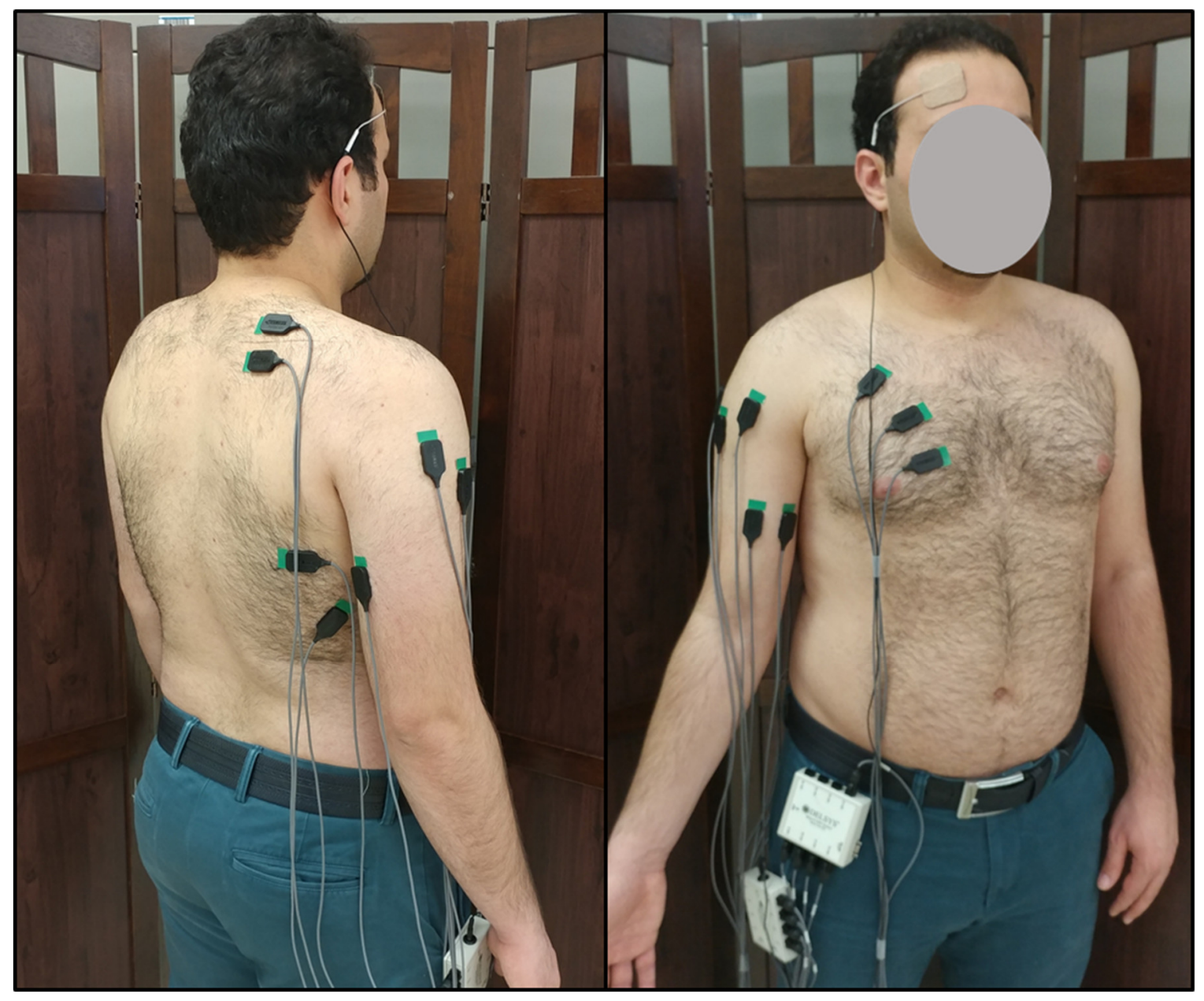

Figure 8. Set of 13 electromyography sensors attached superficial to muscles of interest in this research.

\subsection{Maximum Voluntary Contractions}

After attaching the retroreflective markers and the electromyography sensors, a series of seven maximum voluntary contraction (MVC) exertions were performed three times each, for a total of 21 maximal voluntary contraction trials. The seven maximum voluntary contraction exertions were performed 
Table 2. Muscles that were included in the analysis, including action of the muscle, the location of the muscle, and the placement of the electromyography sensor.

\begin{tabular}{|c|c|c|c|c|}
\hline Muscle & Action & Sensor Placement & Origin & Insertion \\
\hline $\begin{array}{c}\text { Deltoid } \\
\text { (Anterior) }\end{array}$ & $\begin{array}{l}\text { Flexes and } \\
\text { medially } \\
\text { rotates arm }\end{array}$ & $\begin{array}{l}\text { Sitting with arm hanging and the } \\
\text { palm inward, sensor is placed one } \\
\text { finger-width distal and anterior, } \\
\text { oriented along line between } \\
\text { acromion and thumb (SENIAM) }\end{array}$ & \multirow[t]{3}{*}{$\begin{array}{l}\text { Lateral third } \\
\text { of clavicle; } \\
\text { acromion and } \\
\text { spine of } \\
\text { scapula }\end{array}$} & \multirow[t]{3}{*}{$\begin{array}{l}\text { Deltoid } \\
\text { tuberosity of } \\
\text { humerus }\end{array}$} \\
\hline $\begin{array}{c}\text { Deltoid } \\
\text { (Middle) }\end{array}$ & Abducts arm & $\begin{array}{l}\text { Sitting with a stable trunk and arm } \\
\text { hanging, sensor is placed at } \\
\text { biggest bulge along a line between } \\
\text { acromion and lateral epicondyle of } \\
\text { elbow (SENIAM) }\end{array}$ & & \\
\hline $\begin{array}{c}\text { Deltoid } \\
\text { (Posterior) }\end{array}$ & $\begin{array}{l}\text { Extends and } \\
\text { laterally } \\
\text { rotates arm }\end{array}$ & $\begin{array}{l}\text { Sitting with arm hanging and palm } \\
\text { inward, sensor is placed two } \\
\text { finger-widths behind the angle of } \\
\text { acromion, oriented along line } \\
\text { between acromion and little finger } \\
\text { (SENIAM) }\end{array}$ & & \\
\hline $\begin{array}{l}\text { Pectoralis } \\
\text { Major } \\
\text { (Clavicular) }\end{array}$ & $\begin{array}{l}\text { Adducts and } \\
\text { medially } \\
\text { rotates } \\
\text { humerus; } \\
\text { draws scapula } \\
\text { anteriorly and } \\
\text { inferiorly; } \\
\text { flexes humerus }\end{array}$ & $\begin{array}{l}\text { Sensor is placed on the chest wall } \\
\text { at an oblique angle toward the } \\
\text { clavicle, two finger-widths below } \\
\text { the clavicle, just medial to the } \\
\text { axillary fold (Criswell and Cram, } \\
\text { 2011) }\end{array}$ & $\begin{array}{l}\text { Anterior } \\
\text { surface of } \\
\text { medial half of } \\
\text { clavicle }\end{array}$ & $\begin{array}{l}\text { Lateral lip of } \\
\text { intertubercular } \\
\text { groove of } \\
\text { humerus }\end{array}$ \\
\hline $\begin{array}{l}\text { Pectoralis } \\
\text { Major } \\
\text { (Sternal) }\end{array}$ & \multirow{2}{*}{$\begin{array}{l}\text { Adducts and } \\
\text { medially } \\
\text { rotates } \\
\text { humerus; } \\
\text { draws scapula } \\
\text { anteriorly and } \\
\text { inferiorly; } \\
\text { extends } \\
\text { humerus from } \\
\text { flexed position }\end{array}$} & $\begin{array}{l}\text { While rotating the arm medially } \\
\text { against resistance, the sensor is } \\
\text { placed medially to the axillary fold } \\
\text { over the muscle mass that arises, } \\
\text { oriented horizontally (Criswell } \\
\text { and Cram, 2011) }\end{array}$ & \multirow{2}{*}{$\begin{array}{l}\text { Anterior } \\
\text { surface of } \\
\text { sternum, } \\
\text { superior six } \\
\text { costal } \\
\text { cartilages, } \\
\text { aponeurosis } \\
\text { of external } \\
\text { oblique } \\
\text { muscle }\end{array}$} & \\
\hline $\begin{array}{c}\text { Pectoralis } \\
\text { Major } \\
\text { (Abdominal) }\end{array}$ & & $\begin{array}{l}\text { Sensor is placed medially to } \\
\text { axillary fold oriented along a line } \\
\text { between the axillary fold and the } \\
\text { xiphoid process. (Krol, 2007) }\end{array}$ & & \\
\hline $\begin{array}{c}\text { Biceps } \\
\text { Brachium } \\
\text { (Short Head) }\end{array}$ & $\begin{array}{l}\text { Supinates } \\
\text { forearm, and } \\
\text { when it is } \\
\text { supine, flexes } \\
\text { forearm; } \\
\text { resists } \\
\text { dislocation of } \\
\text { the shoulder }\end{array}$ & $\begin{array}{l}\text { Sitting with elbow flexed at right } \\
\text { angle and dorsal side of forearm in } \\
\text { a horizontal downward position, } \\
\text { sensor is placed two finger-widths } \\
\text { medial of line between medial } \\
\text { acromion and fossa cubit, } 1 / 3 \text { from } \\
\text { the fossa cubit (SENIAM) }\end{array}$ & $\begin{array}{l}\text { Tip of } \\
\text { coracoid } \\
\text { process of } \\
\text { scapula }\end{array}$ & $\begin{array}{l}\text { Tuberosity of } \\
\text { radius and } \\
\text { fascia of } \\
\text { forearm via } \\
\text { bicipital } \\
\text { aponeurosis }\end{array}$ \\
\hline
\end{tabular}




\begin{tabular}{|c|c|c|c|c|}
\hline Muscle & Action & Sensor Placement & Origin & Insertion \\
\hline $\begin{array}{c}\text { Biceps } \\
\text { Brachium } \\
\text { (Long Head) }\end{array}$ & $\begin{array}{l}\text { Supinates } \\
\text { forearm, and } \\
\text { when it is } \\
\text { supine, flexes } \\
\text { forearm }\end{array}$ & $\begin{array}{l}\text { Sitting with elbow flexed at right } \\
\text { angle and dorsal side of forearm in } \\
\text { a horizontal downward position, } \\
\text { sensor is placed two finger-widths } \\
\text { lateral of line between medial } \\
\text { acromion and fossa cubit, } 1 / 3 \text { from } \\
\text { fossa cubit (SENIAM) }\end{array}$ & $\begin{array}{l}\text { Supraglenoid } \\
\text { tubercle of } \\
\text { scapula }\end{array}$ & \\
\hline $\begin{array}{c}\text { Triceps } \\
\text { (Long Head) }\end{array}$ & $\begin{array}{l}\text { Extends } \\
\text { forearm; } \\
\text { resists } \\
\text { dislocation of } \\
\text { humerus }\end{array}$ & $\begin{array}{l}\text { Sitting with shoulder abducted } 90 \\
\text { degrees and arm flexed } 90 \text { degrees } \\
\text { with palm downward, sensor is } \\
\text { placed two finger-widths lateral of } \\
\text { the midpoint of a line between the } \\
\text { posterior crista of the acromion } \\
\text { and the olecranon (SENIAM) }\end{array}$ & $\begin{array}{l}\text { Infraglenoid } \\
\text { tubercle of } \\
\text { scapula }\end{array}$ & $\begin{array}{l}\text { Proximal end } \\
\text { of olecranon } \\
\text { ulna and } \\
\text { fascia of } \\
\text { forearm }\end{array}$ \\
\hline $\begin{array}{l}\text { Latissimus } \\
\text { Dorsi }\end{array}$ & $\begin{array}{l}\text { Extends, } \\
\text { adducts, and } \\
\text { medially } \\
\text { rotates } \\
\text { humerus; } \\
\text { raises body } \\
\text { toward arms } \\
\text { during } \\
\text { climbing }\end{array}$ & $\begin{array}{l}\text { Sensor is placed } 4 \mathrm{~cm} \text { below the } \\
\text { inferior spine of the scapula, } \\
\text { halfway between the spine and the } \\
\text { lateral edge of the body, oriented } \\
\text { obliquely at approximately } 25 \\
\text { degrees (Criswell and Cram, } \\
\text { 2011) }\end{array}$ & $\begin{array}{l}\text { Spinous } \\
\text { processes of } \\
\text { inferior } 6 \\
\text { thoracic } \\
\text { vertebrae, } \\
\text { thoracolumbar } \\
\text { fascia, iliac } \\
\text { crest, and } \\
\text { inferior } 3 \text { or } 4 \\
\text { ribs }\end{array}$ & $\begin{array}{l}\text { Floor of } \\
\text { intertubercular } \\
\text { groove of } \\
\text { humerus }\end{array}$ \\
\hline Infraspinatus & $\begin{array}{l}\text { Laterally } \\
\text { rotate arm; } \\
\text { help hold } \\
\text { humeral head } \\
\text { in glenoid } \\
\text { cavity of } \\
\text { scapula } \\
\end{array}$ & $\begin{array}{l}\text { Sensor is placed parallel to and } \\
\text { approximately } 4 \mathrm{~cm} \text { below the } \\
\text { lateral aspect of the spine of the } \\
\text { scapula, over the infraspinous } \\
\text { fossa of the scapula (Criswell and } \\
\text { Cram, 2011) }\end{array}$ & $\begin{array}{l}\text { Infraspinous } \\
\text { fossa of } \\
\text { scapula }\end{array}$ & $\begin{array}{l}\text { Middle facet } \\
\text { of greater } \\
\text { tubercle of } \\
\text { humerus }\end{array}$ \\
\hline Supraspinatus & $\begin{array}{l}\text { Initiates and } \\
\text { assists Deltoid } \\
\text { in abduction of } \\
\text { arms; help } \\
\text { hold humeral } \\
\text { head in } \\
\text { glenoid cavity } \\
\text { of scapula }\end{array}$ & $\begin{array}{l}\text { Sensor is placed parallel to and } \\
\text { approximately } 4 \mathrm{~cm} \text { sbove the } \\
\text { lateral aspect of the spine of the } \\
\text { scapula, over the supraspinous } \\
\text { fossa of the scapula (Criswell and } \\
\text { Cram, 2011) }\end{array}$ & $\begin{array}{l}\text { Supraspinous } \\
\text { fossa of } \\
\text { scapula }\end{array}$ & $\begin{array}{l}\text { Superior facet } \\
\text { of greater } \\
\text { tubercle of } \\
\text { humerus }\end{array}$ \\
\hline Teres Major & $\begin{array}{l}\text { Adducts and } \\
\text { medially } \\
\text { rotates arm }\end{array}$ & $\begin{array}{l}\text { Sensor is placed three finger- } \\
\text { widths above the inferior angle of } \\
\text { the scapula, along the lateral } \\
\text { border, oriented horizontally ( } \mathbf{X u} \\
\text { et al., 2014) }\end{array}$ & $\begin{array}{l}\text { Posterior } \\
\text { surface of } \\
\text { inferior angle } \\
\text { of scapula }\end{array}$ & $\begin{array}{l}\text { Medial lip of } \\
\text { intertubercular } \\
\text { groove of } \\
\text { humerus }\end{array}$ \\
\hline
\end{tabular}


as described in the following subsections. Appendix D contains individual pages for each maximum voluntary contraction with images of the posture and rehabilitation machine setup that were used as guidance to ensure maximum voluntary contraction tasks were performed the same by each participant.

For each of these exertions, the participant was directed to slowly build up to their maximum exertion over a period of three seconds and maintain that maximal exertion to the best of their ability for five seconds. A two-minute rest period was provided between each repetition. After completing all maximum voluntary contraction trials, the participant began the experimental tasks.

2.8.1 Maximum Voluntary Contraction \#1: With the shoulder abducted to $90^{\circ}$ in the scapular place and $45^{\circ}$ internally rotated and elbow extended, the participant abducted the arm with maximum force against resistive force applies at the wrist. This exertion targeted the Deltoid, Pectoralis Major, and Supraspinatus (Kelly et al., 1996).

2.8.2 Maximum Voluntary Contraction \#2: With the shoulder abducted to $125^{\circ}$, the participant resisted a force applied above the elbow toward the inferior angle of the scapula to de-rotate the scapula. This exertion targeted the Anterior Deltoid, Middle Deltoid, Infraspinatus, and Supraspinatus (Xu et al., 2014).

2.8.3 Maximum Voluntary Contraction \#3: With the shoulder abducted to $90^{\circ}$ in the scapular plane and $90^{\circ}$ elbow flexion, the participant internally rotated with maximal force against a resistive force applied at the wrist. This exertion targeted the Latissimus Dorsi (Xu et al., 2014).

2.8.4 Maximum Voluntary Contraction \#4: With the elbow flexed to $90^{\circ}$, the participant flexed the arm with maximum force against resistive force at the wrist. This exertion targeted the Biceps Brachii (Xu et al., 2014).

2.8.5 Maximum Voluntary Contraction \#5: With the elbow flexed to $90^{\circ}$, the participant extended their elbow with maximum force against resistive force at the wrist. This exertion targeted the Triceps (Xu et al., 2014). 
2.8.6 Maximum Voluntary Contraction \#6: With neutral posture of the shoulder and $90^{\circ}$ of elbow flexion, the participant internally rotated with maximal force against resistive force applied at the wrist. This exertion targeted the Pectoralis Major (Kelly et al., 1996).

2.8.7 Maximum Voluntary Contraction \#7: With the shoulder abducted to $45^{\circ}$ and $90^{\circ}$ of elbow flexion, the participant internally rotated their arm against a resistive force at the wrist. This exertion targeted the Teres Major (Xu et al., 2014).

\subsection{Experimental Tasks}

The experimental tasks consisted of a single lifting task performed while holding three different weights $(2.5 \mathrm{lbs}, 5 \mathrm{lbs}$, and $10 \mathrm{lbs})$. The participant held the weight with the arm to their side. When told to begin and while maintaining a straight arm, the participant lifted the weight up to shoulder height at a steady pace over a period of three seconds in the frontal plane (Figure 9). The participant held the weight in that position for a period of five seconds, then set the weight down. Metronome software was used to provide a tone every second to provide audible feedback to the participant and assist with timing. A two-minute rest period will be provided between trials.

\subsection{Data Processing}

2.10.1 Motion Capture Data Processing: When two or more cameras capture reflections from the same marker, the software can determine the three-dimensional coordinates of the marker $(\mathrm{X}, \mathrm{Y}, \mathrm{Z})$ in the global coordinate frame that is defined during system calibration. Motion capture data was collected at $100 \mathrm{~Hz}$. The accompanying software, Vicon Nexus 1.8.1, was used to label each marker with its identifier (i.e. left front head markers is LFHD) and fill any gaps in the data where a marker may be obscured. The resulting data was used to drive the motion of a musculoskeletal model in AnyBody Modeling System. 


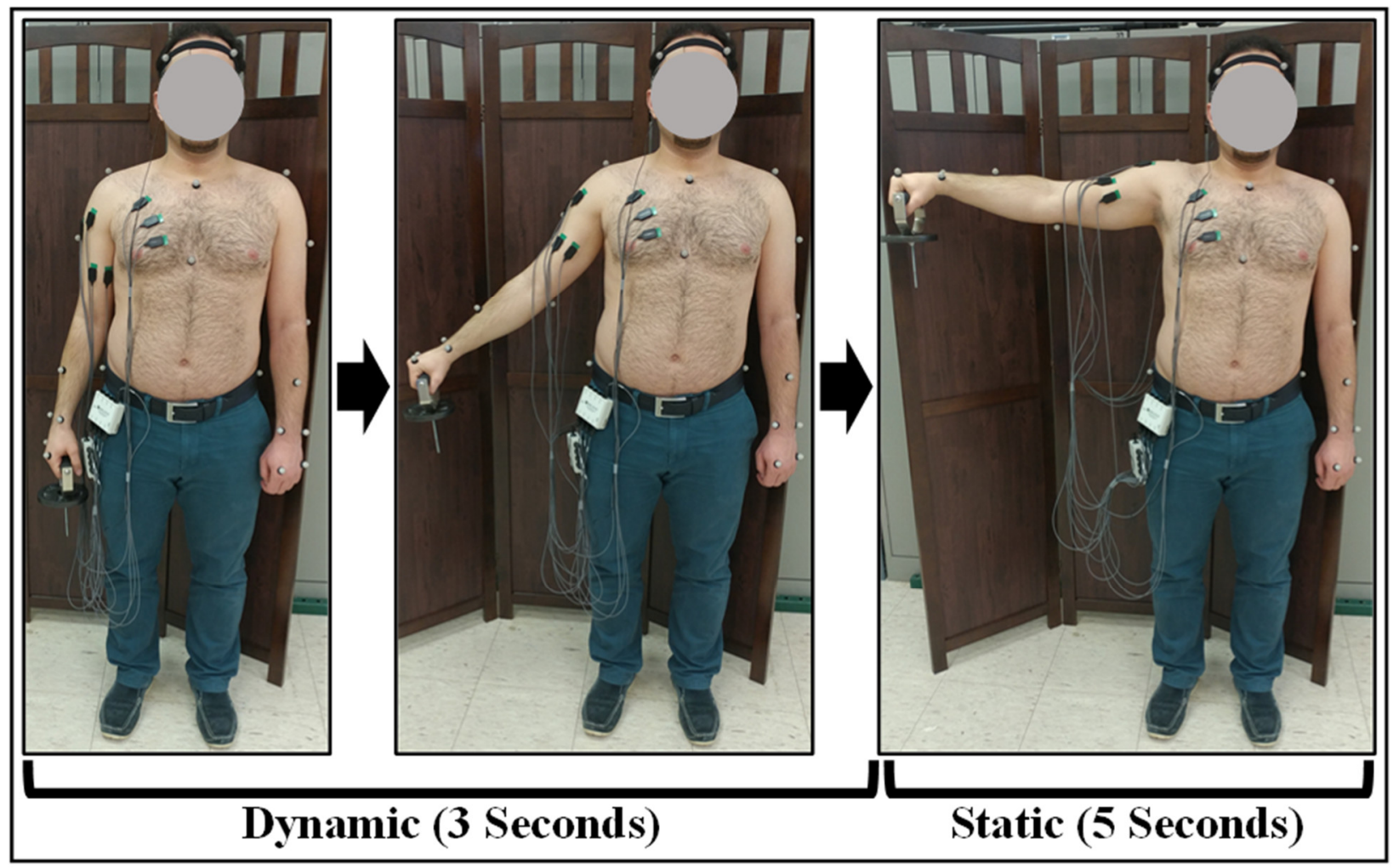

Figure 9. Progression for the experimental task performed by the participants. Participant is lifting a 2.5 pound weight.

There are two primary steps involved in processing motion capture data in Vicon Nexus. First, the markers must be labeled (Figure 10a). Each marker has a short label/identifier specified in a template file. When labeling a marker, the label is applied to every proceeding and preceding frame of the trajectory until a gap is encountered. Since a gap signifies that the marker can no longer be tracked, the marker's trajectory after the gap cannot automatically be associated with the prior labeled trajectory, therefore the marker must be labeled again when it reappears after the gap. Once every unlabeled trajectory was labeled, the next step was filling in the gaps (Figure 10b). Vicon Nexus 1.8.1 has two methods for filling in the gaps; spline fill and pattern fill. 


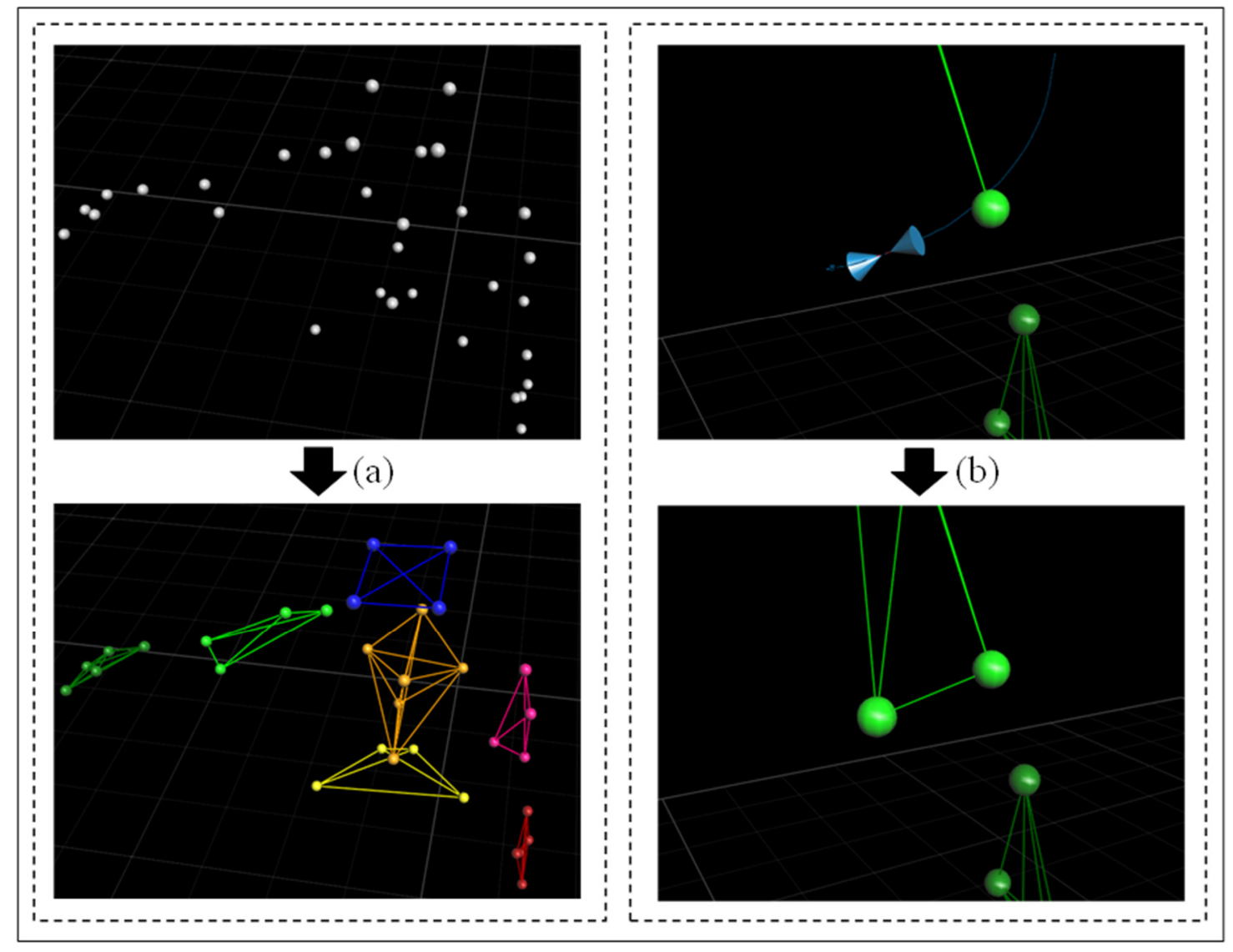

Figure 10. Vicon Nexus data processing steps. (a) Unlabeled markers are labeled with their unique identifying label. (b) Gaps in the marker data are filled.

The spline fill function generates a quantic spline using marker locations immediately before and after the gap as seed data and the missing data is filled using the interpolated values. The spline fill function is most appropriate for filling small gaps in smooth consistent motions. As the gap lengthens, interpolated values using the spline fill become increasingly erratic resulting in a drastic reduction of data accuracy. The pattern fill function requires a source marker with a similar trajectory as the marker with the gap in the data. A donor trajectory from the course marker covering the same frames is used to interpolate the trajectory of the missing data. The donor trajectory must not have any missing data, or the function will fail. Using a source marker with a dissimilar trajectory will result in inaccurate data. Regardless of which gap filling function is used, visual inspection of the interpolated trajectory is important to ensure the function 
performed as expected and that the interpolated trajectory closely matches the actual motion. Gap filling can introduce errors, so special attention was given when placing the cameras to provide the best capture of the markers and to ensure that gaps in the data were minimal.

Once all gaps in the data were filled, the marker trajectories and electromyography data were exported. Finally, frame numbers of the beginning and end of the dynamic portion (beginning of trial until arm was parallel with the ground) of the trial and the static portion (while the arm was maintained parallel to the ground) of the trial were noted.

2.10.2 Musculoskeletal Modeling: Each experimental task was modeled in AnyBody modeling system using each available muscle recruitment strategy available in AnyBody Modeling System 6.0. In the model, most muscles are represented by multiple musculotendon units (Figure 11) to deal with branching muscles, such as the Latissimus Dorsi and Pectoralis Major, and muscles with multiple origins/insertions, such as the Biceps Brachii. In the model, each of these musculotendon segments are treated as individual muscles, which represented a challenge in the analysis, i.e. how to compare one EMG value to the multiple outputs from a modeled-muscle that was comprised of multiple segments. The way this was handled depended on the number of EMG sensors on the muscle and the number of components comprising its modeled representation.

The Deltoid and Pectoralis Major muscles each had 3 electromyography sensors. For these muscles, the modeled musculotendon units that were deep to (beneath) the location of the sensors were grouped. The Deltoid muscle in the model was comprised of 12 musculotendon units with the majority being located more anteriorly. Due to this layout, the six units that were most anterior were selected to represent the Anterior Deltoid, the next 3 units represented the Middle Deltoid, and the three posterior musculotendon units represented the Posterior Deltoid. The Pectoralis Major muscle in the model was comprised of 10 musculotendon units to represent, with a resulting breakup of five, three, and two musculotendon units to represent the Clavicular, Sternal, and Abdominal Pectoralis Major muscles, respectively. 


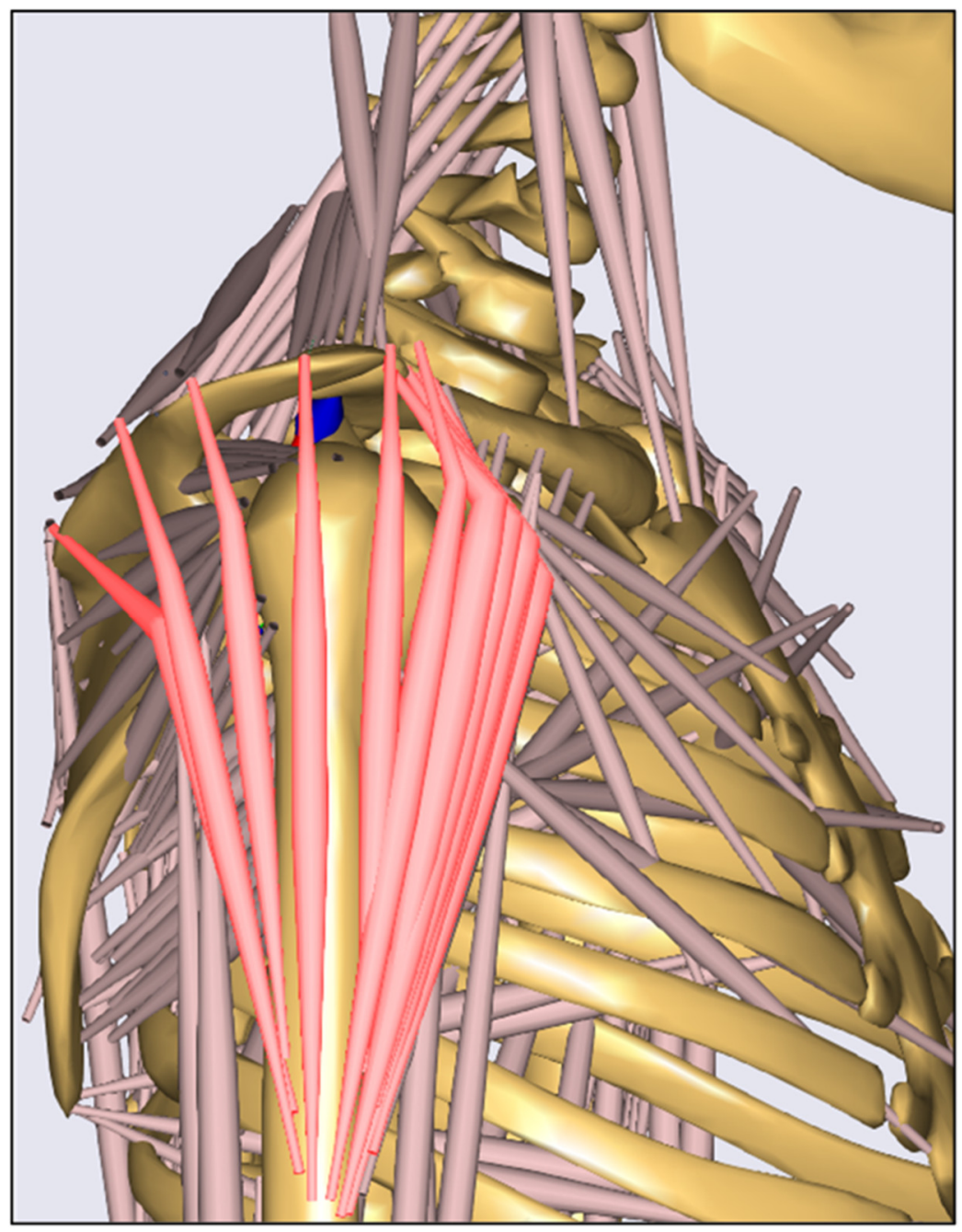

Figure 11. Musculotendon units that comprise the Deltoid.

The Biceps Brachii muscle had two electromyography sensors and is represented by two musculotendon units in the model. Logically, it would make sense to have each of these musculotendon units represent one of the two musculotendon units, functionally, this does not provide an accurate representation. Due to the optimization, it was common for only one of the two musculotendon units to be contributing, especially during low-force exertions, and often the roles would swap and the non-working muscle would begin performing the necessary work while the formerly contributing muscle would become non-working. Therefore, the two musculotendon units were used to represent both the Medial Biceps Brachii and Lateral Biceps Brachii. 
The remaining muscles, the Triceps, Latissimus Dorsi, Infraspinatus, Supraspinatus, and Teres Major each had a single electromyography sensor. Therefore, all musculotendon units comprising a muscle in the model were used to represent that muscle. Next, the muscle activity data from the multiple musculotendon units was summarized into one comparable output for each electromyography sensor. Since the musculotendon units representing a muscle often had different cross-sectional areas, which were multiplied by a constant factor of $115 \mathrm{~N} / \mathrm{cm}^{2}$ to determine the maximum force capabilities of that musculoskeletal unit, the contributions of each musculotendon unit were weighted based on its maximum force capability. Cross-sectional areas were scaled to the anthropometry of the participants, so these numbers were not the same for all participants, however the weighting remained the same when compared between two participants with different physical builds, so for the sake of simplicity one set of values was used for all participants. The musculotendon units that represented each muscle, maximum force capability values used in the analysis, and the weighting of the musculotendon units is given in Table 3.

Table 3. Muscles that were studies in this research, their representative musculotendon units from the model, and each musculotendon unit's maximum strength and weighting.

\begin{tabular}{|c|c|c|c|}
\hline Muscle & Musculotendon Units & Max Strength & Weight \\
\hline \multirow[t]{7}{*}{ Anterior Deltoid } & deltoideus_clavicular_part_1 & 218.09 & 0.17 \\
\hline & deltoideus_clavicular_part_2 & 233.31 & 0.19 \\
\hline & deltoideus_clavicular_part_3 & 253.60 & 0.20 \\
\hline & deltoideus_clavicular_part_4 & 162.30 & 0.13 \\
\hline & deltoideus_clavicular_part_5 & 182.59 & 0.15 \\
\hline & deltoideus clavicular part 6 & 197.80 & 0.16 \\
\hline & Total: & 1247.69 & \\
\hline \multirow[t]{4}{*}{ Middle Deltoid } & deltoideus_scapular_part_4 & 281.49 & 0.33 \\
\hline & deltoideus_scapular_part_5 & 281.49 & 0.33 \\
\hline & deltoideus_scapular_part_6 & 281.49 & 0.33 \\
\hline & Total: & 844.47 & \\
\hline
\end{tabular}




\begin{tabular}{|c|c|c|c|}
\hline Muscle & Musculotendon Units & Max Strength & Weight \\
\hline \multirow[t]{4}{*}{ Posterior Deltoid } & deltoideus_scapular_part_1 & 342.35 & 0.33 \\
\hline & deltoideus_scapular_part_2 & 342.35 & 0.33 \\
\hline & deltoideus scapular part 3 & 342.35 & 0.33 \\
\hline & Total: & 1027.06 & \\
\hline \multirow[t]{6}{*}{ Pectoralis Major (Clavicular) } & pectoralis_major_clavicular_part_1 & 140.65 & 0.18 \\
\hline & pectoralis_major_clavicular_part_2 & 155.07 & 0.20 \\
\hline & pectoralis_major_clavicular_part_3 & 155.07 & 0.20 \\
\hline & pectoralis_major_clavicular_part_4 & 155.07 & 0.20 \\
\hline & pectoralis major clavicular part 5 & 155.07 & 0.20 \\
\hline & Total: & 760.94 & \\
\hline \multirow[t]{4}{*}{ Pectoralis Major (Sternal) } & pectoralis_major_thoracic_part_3 & 155.07 & 0.33 \\
\hline & pectoralis_major_thoracic_part_4 & 155.07 & 0.33 \\
\hline & pectoralis_major_thoracic_part_5 & 155.07 & 0.33 \\
\hline & $\begin{array}{c}\text { Total: } \\
\end{array}$ & 465.22 & \\
\hline \multirow{3}{*}{$\begin{array}{l}\text { Pectoralis Major } \\
\text { (Abdominal) }\end{array}$} & pectoralis_major_thoracic_part_1 & 155.07 & 0.50 \\
\hline & pectoralis major_thoracic part 2 & 155.07 & 0.50 \\
\hline & Total: & 310.15 & \\
\hline \multirow[t]{3}{*}{ Bicep Brachii (Medial) } & biceps_brachii_caput_breve & 218.09 & 0.49 \\
\hline & biceps_brachii_caput_longum & 225.70 & 0.51 \\
\hline & Total: & 443.79 & \\
\hline \multirow[t]{3}{*}{ Bicep Brachii (Lateral) } & biceps_brachii_caput_breve & 218.09 & 0.49 \\
\hline & biceps_brachii_caput_longum & 225.70 & 0.51 \\
\hline & $\begin{array}{ll}\text { Total: } \\
\end{array}$ & 443.79 & \\
\hline \multirow[t]{3}{*}{ Triceps } & Triceps_LH_1 & 356.27 & 0.50 \\
\hline & Triceps LH 2 & 356.27 & 0.50 \\
\hline & Total: & 712.55 & \\
\hline \multirow[t]{6}{*}{ Latissimus Dorsi } & latissimus_dorsi_1 & 216.06 & 0.20 \\
\hline & latissimus_dorsi_2 & 216.06 & 0.20 \\
\hline & latissimus_dorsi_3 & 216.06 & 0.20 \\
\hline & latissimus_dorsi_4 & 216.06 & 0.20 \\
\hline & latissimus_dorsi_5 & 216.06 & 0.20 \\
\hline & Total: & 1080.32 & \\
\hline
\end{tabular}




\begin{tabular}{|c|c|c|c|c|}
\hline Muscle & Musculotendon Units & & Max Strength & Weight \\
\hline \multirow[t]{7}{*}{ Infraspinatus } & infraspinatus_1 & & 172.70 & 0.17 \\
\hline & infraspinatus_2 & & 172.70 & 0.17 \\
\hline & infraspinatus_3 & & 172.70 & 0.17 \\
\hline & infraspinatus_4 & & 172.70 & 0.17 \\
\hline & infraspinatus_5 & & 172.70 & 0.17 \\
\hline & infraspinatus_6 & & 172.70 & 0.17 \\
\hline & & Total: & 1036.19 & \\
\hline \multirow[t]{7}{*}{ Supraspinatus } & supraspinatus_1 & & 79.12 & 0.17 \\
\hline & supraspinatus_2 & & 79.12 & 0.17 \\
\hline & supraspinatus_3 & & 79.12 & 0.17 \\
\hline & supraspinatus_4 & & 79.12 & 0.17 \\
\hline & supraspinatus_5 & & 79.12 & 0.17 \\
\hline & supraspinatus_6 & & 79.12 & 0.17 \\
\hline & & Total: & 474.73 & \\
\hline \multirow[t]{7}{*}{ Teres Major } & teres_major_1 & & 232.80 & 0.17 \\
\hline & teres_major_2 & & 232.80 & 0.17 \\
\hline & teres_major_3 & & 232.80 & 0.17 \\
\hline & teres_major_4 & & 232.80 & 0.17 \\
\hline & teres_major_5 & & 232.80 & 0.17 \\
\hline & teres major 6 & & 232.80 & 0.17 \\
\hline & & Total: & 1396.81 & \\
\hline
\end{tabular}

At each timeframe, the output of interest from the musculoskeletal model was predicted muscle activity (\%MA) of each musculotendon unit, calculated as the percentage of the maximum force capability of the musculotendon unit that was used to perform the task being modeled based on the optimization results for the selected muscle recruitment strategy. Remaining data processing steps, i.e. electromyography data processing and calculation of correlation and RMSE values, were performed using a script coded in MATLAB (Appendix E) and are detailed in the following sections.

2.10.3 Electromyography Data Processing: Electromyography data from the maximum voluntary contraction trials and the experimental task trials was first purged of heartbeat and other artifacts using a notch filter to eliminate frequencies under $60 \mathrm{~Hz}$ (Figure 12a). The data was then demeaned by calculating 
the mean value of the data and subtracting that calculated value from each data point, resulting in a mean of 0 for the data (Figure 12b). The data was then full-wave rectified by taking the absolute value of each data point (Figure 12c). Next, the data was low-pass filtered using a digital $4^{\text {th }}$ order Butterworth filter with a $4 \mathrm{~Hz}$ cut-off to generate a linear envelope (Figure 12d). Additionally, to match sample rates between the motion capture and musculoskeletal modeling data, the processed electromyography data was resampled from $1000 \mathrm{~Hz}$ to $100 \mathrm{~Hz}$.

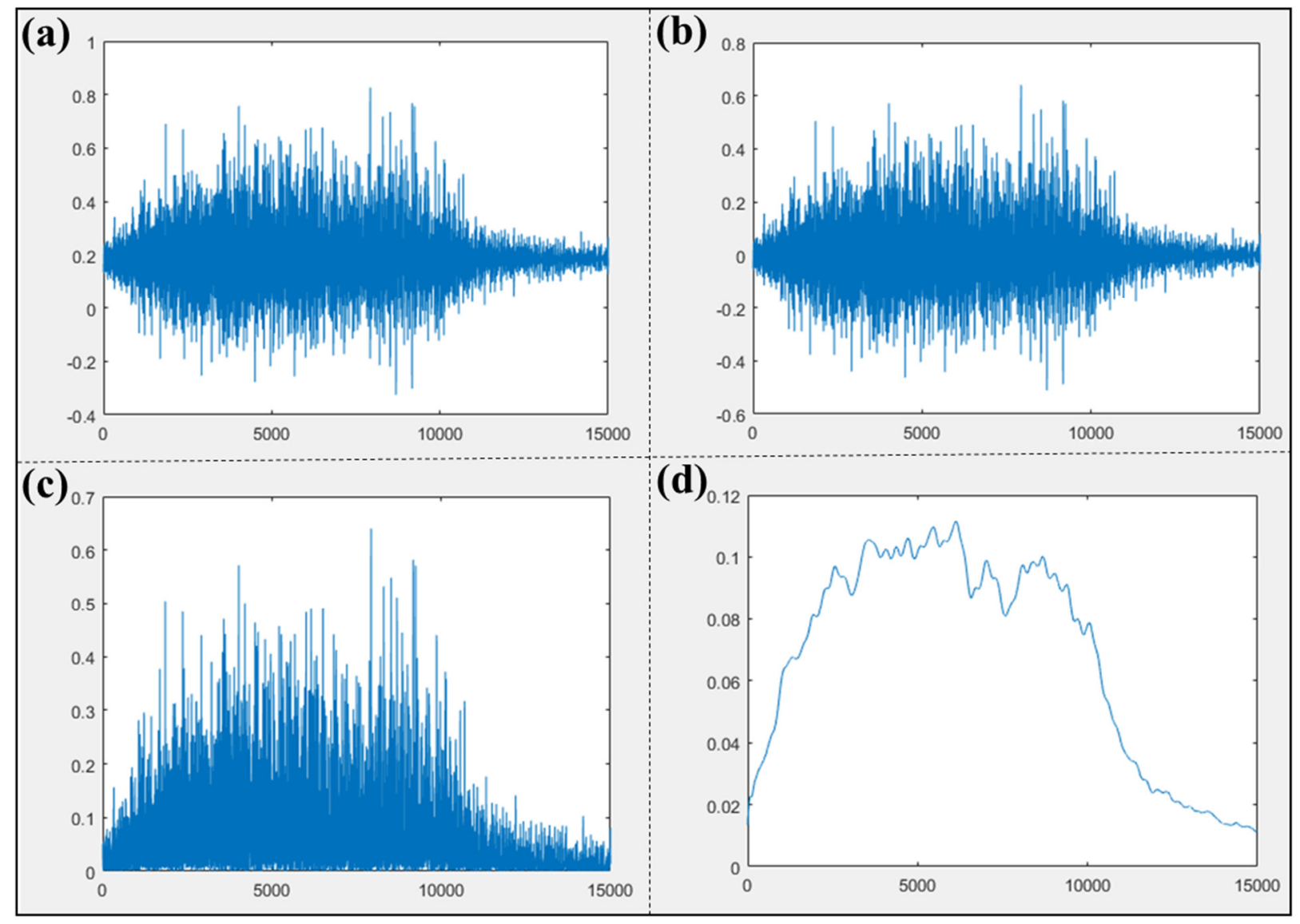

Figure 12. Electromyography data processing steps. (a) Raw electromyography data. (b) Notch filtered and demeaned data. (c) Full-wave rectified data. (d) Butterworth filtered data forming a linear envelope.

While each MVC task was designed to maximally engage one or more specific muscles, initial analysis showed that, in certain circumstances, a muscle was engaged more by a different MVC exertion than the one assigned. Therefore, after processing the MVC data, the MVC data across all trials was 
aggregated for each muscle and the peak muscle activity value for each muscle was treated as the maximum and was used in experimental trials to normalize the electromyography data.

The electromyography data for the experimental trials was normalized using the previously calculated values by dividing each data point from the experimental trial data by the maximum voluntary contraction value for the corresponding muscle, called \%MVC values. This normalization process accounted for differences in participant characteristics and inter-participant electrode placement, which allowed the electromyography data to be compared between participants.

2.10.4 Correlation and RMSE Calculation: At each timeframe, for each of the 13 muscles, the \%MA value of each musculotendon unit was multiplied by its maximum force capability to determine the amount of force exerted by each unit. The forces exerted by the musculotendon units of the muscle were added together to determine how much force was produced by the muscle. The maximum force capability of the muscle was divided by the force produced by the muscle to determine a \%MA value for the overall muscle, allowing a more direct comparison with the \%MVC values for each muscle. Further analysis of the static and dynamic portions of the task are described separately in the following subsections due to differences in the analysis.

2.10.4.1 Dynamic Analysis: The dynamic portion of the tasks were compared directly by calculating Pearson correlation (Equation 4) and RMSE (Equation 5) values between the \%MA and \%MVC values of each muscle for each recruitment strategy were calculated for the three experimental trials.

2.10.4.2 Static Analysis: Since there was minimal motion during the static portion of the task, the \%MA values from the model and the \%MVC values from the electromyography data remained near-constant. Pearson correlation, in this case, would have created artificially high correlation values that would be difficult to compare. Therefore, Spearman Rank Correlation (Equation 6) was used for the static portion of the data. For each recruitment strategy for each muscle, the mean \%MVC value and mean \%MA value will be calculated. The total force exerted by all muscles together was calculated by multiplying the \%MVC and 
$\%$ MA values by the maximum force capability values for each muscle. The total force exerted by each muscle was divided by the total maximum force capability of all 13 muscles combined to determine the proportion that each muscle contributed to the total overall force. For each recruitment strategy, Spearman Rank Correlation values were calculated between the 13 proportion values calculated from the electromyography data and 13 proportion values calculated from the model output data.

$r=\frac{\sum_{i=1}^{n}\left(x_{i}-\bar{x}\right)\left(y_{i}-\bar{y}\right)}{\sqrt{\sum_{i=1}^{n}\left(x_{i}-\bar{x}\right)^{2}} \sqrt{\sum_{i=1}^{n}\left(y_{i}-\bar{y}\right)^{2}}}$

where:

$\mathrm{n}=$ frame-length of data,

$x_{i}=\% \mathrm{MVC}$ values from electromyography, and

$y_{i}=\% \mathrm{MA}$ values from model.

$R M S E=\sqrt{\frac{\sum_{i=1}^{n}\left(\hat{y}_{i}-y_{i}\right)^{2}}{n}}$

(Equation 5)

where:

$\mathrm{n}=$ frame-length of data,

$\hat{y}=\%$ MA values from model, and

$\mathrm{y}=\% \mathrm{MVC}$ values from electromyography.

$\rho=1-\frac{6 \sum d_{i}^{2}}{n\left(n^{2}-1\right)}$

(Equation 6)

where:

$n=$ number of samples

$d_{i}=$ difference in rank of the $i$ th muscle between model and electromyography data. 


\subsection{Results}

2.11.1 Dynamic Analysis: Mean correlation for each recruitment strategy across all muscles and weight conditions ranged from 0.4939 to 0.5643 for the MMS and Poly4 strategies, respectively. Corresponding RMSE values ranged from 0.1458 to 0.1669 for MMS and Quad strategies, respectively (Table 4). Based on this analysis it appears that Poly4 resulted in the highest mean correlation, while maintaining a relatively low mean RMSE value (Figure 13).

Table 4. Overall mean correlation and RMSE values for each recruitment strategy for the dynamic analysis.

\begin{tabular}{|l|r|r|}
\hline \multicolumn{1}{|c|}{ Recruitment Strategy } & \multicolumn{1}{c|}{ Correlation } & RMSE (\%) \\
\hline Quadratic (Quad) & 0.5146 & 0.1669 \\
\hline 3rd-order Polynomial (Poly3) & 0.5621 & 0.1599 \\
\hline 4th-order Polynomial (Poly4) & 0.5643 & 0.1544 \\
\hline 5th-order Polynomial (Poly5) & 0.5353 & 0.1514 \\
\hline Min/Max (MMS) & 0.4939 & 0.1458 \\
\hline Composite (Comp) & 0.5300 & 0.1661 \\
\hline
\end{tabular}

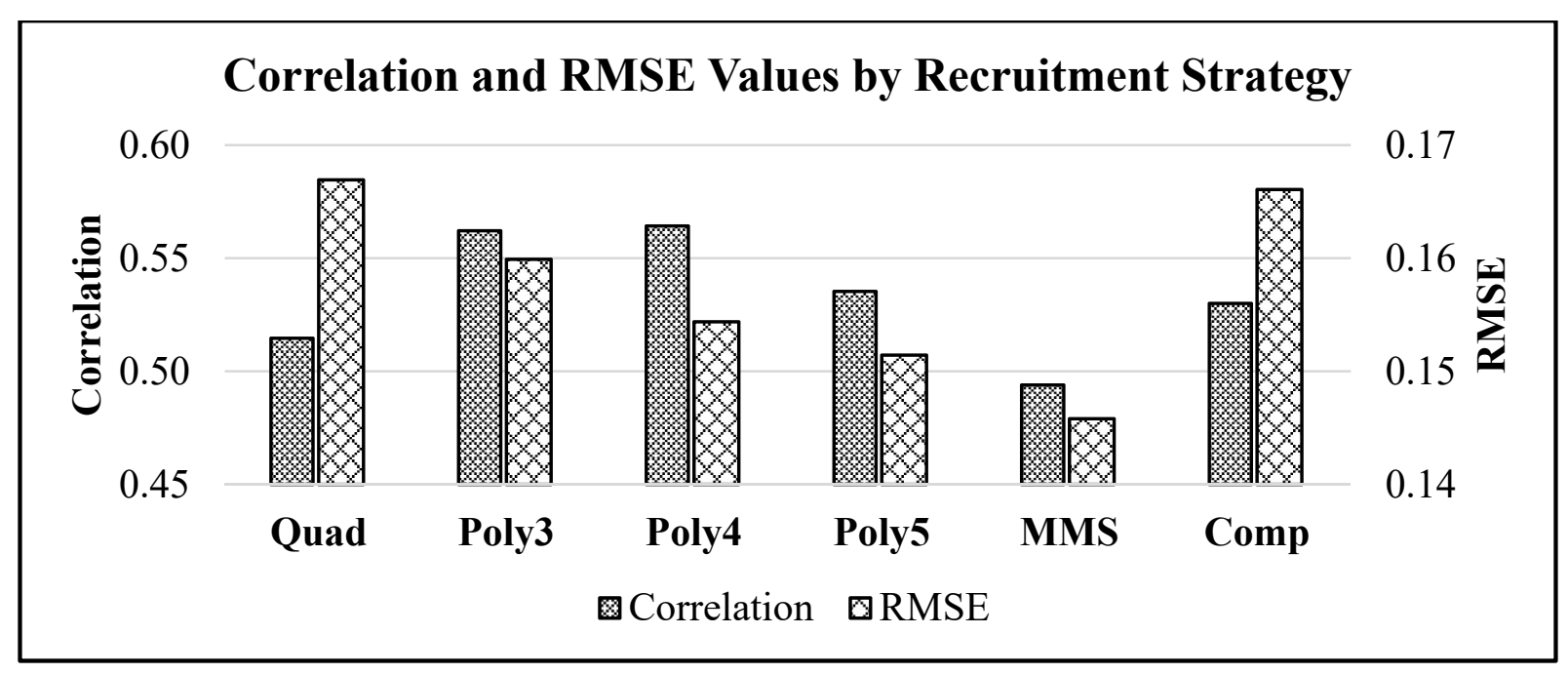

Figure 13. Mean correlation values and mean RMSE values found for each recruitment strategy for the dynamic analysis. 
However, when the correlation values are stratified by muscle and recruitment strategy, the range of correlation values is much greater (Table 5), ranging from -0.3822 to 0.9269 for the Latissimus Dorsi with the MMS recruitment strategy and Anterior Deltoid with the Poly4 recruitment strategy.

Table 5. Correlation values stratified by muscle and recruitment strategy for the dynamic analysis.

\begin{tabular}{|l|c|c|c|c|c|c|}
\hline Muscle & Quad & Poly3 & Poly4 & Poly5 & MMS & Comp \\
\hline Anterior Deltoid & 0.9040 & 0.9257 & 0.9269 & 0.9252 & 0.9100 & 0.9179 \\
\hline Middle Deltoid & 0.8106 & 0.8300 & 0.8344 & 0.8354 & 0.8232 & 0.8298 \\
\hline Posterior Deltoid & 0.8321 & 0.8332 & 0.8341 & 0.8347 & 0.8360 & 0.8312 \\
\hline Pec. Major (Clavicular) & 0.1465 & 0.2308 & 0.2096 & 0.0677 & 0.1448 & 0.2481 \\
\hline Pec. Major (Sternal) & 0.1363 & 0.2087 & 0.1857 & 0.0823 & 0.1681 & 0.2308 \\
\hline Pec. Major (Abdominal) & 0.0975 & 0.1471 & 0.1303 & 0.0224 & 0.0599 & 0.1943 \\
\hline Biceps (Medial) & 0.5352 & 0.6423 & 0.6503 & 0.6539 & 0.3828 & 0.6221 \\
\hline Biceps (Lateral) & 0.4612 & 0.5731 & 0.5726 & 0.5690 & 0.3449 & 0.5476 \\
\hline Triceps & 0.6456 & 0.6607 & 0.6578 & 0.6720 & 0.6296 & 0.6198 \\
\hline Latissimus Dorsi & -0.1034 & 0.0017 & -0.0173 & -0.1053 & -0.3822 & 0.0306 \\
\hline Infraspinatus & 0.8983 & 0.8970 & 0.8941 & 0.8935 & 0.8868 & 0.8883 \\
\hline Supraspinatus & 0.8161 & 0.8385 & 0.8424 & 0.8445 & 0.8424 & 0.7024 \\
\hline Teres Major & 0.5099 & 0.5191 & 0.6145 & 0.6641 & 0.7751 & 0.2275 \\
\hline
\end{tabular}

Poor correlation values were consistent across all recruitment strategies for the Clavicular, Sternal, and Abdominal Pectoralis Major muscles and Latissimus Dorsi. These muscles are generally more active with forceful adduction exertions of the shoulder, while the lifting tasks involved forceful abduction exertions of the shoulder. Therefore, the modeled muscle activity of the musculotendon units for these muscles is minimal. For instance, for one participant during the dynamic portion of the 10-lb lifting task, the percent muscle activity of the five Latissimus Dorsi musculotendon units peaked at 1.04x10-8 (Figure 14), which is negligible. Additionally, the $\% \mathrm{MVC}$ values for that same condition ranged from 0.0504 to 0.1556 , and the changes in the values were more gradual and less frequent than for the corresponding percent muscle activity value (Figure 15), resulting in the low correlations. The remaining 9 muscles had 
correlation values that ranged from acceptable to excellent. Correlations for the Teres Major, Medial and Lateral Biceps Brachii, and Triceps muscles were comparable (Range: 0.3449-0.7751) to the 0.580

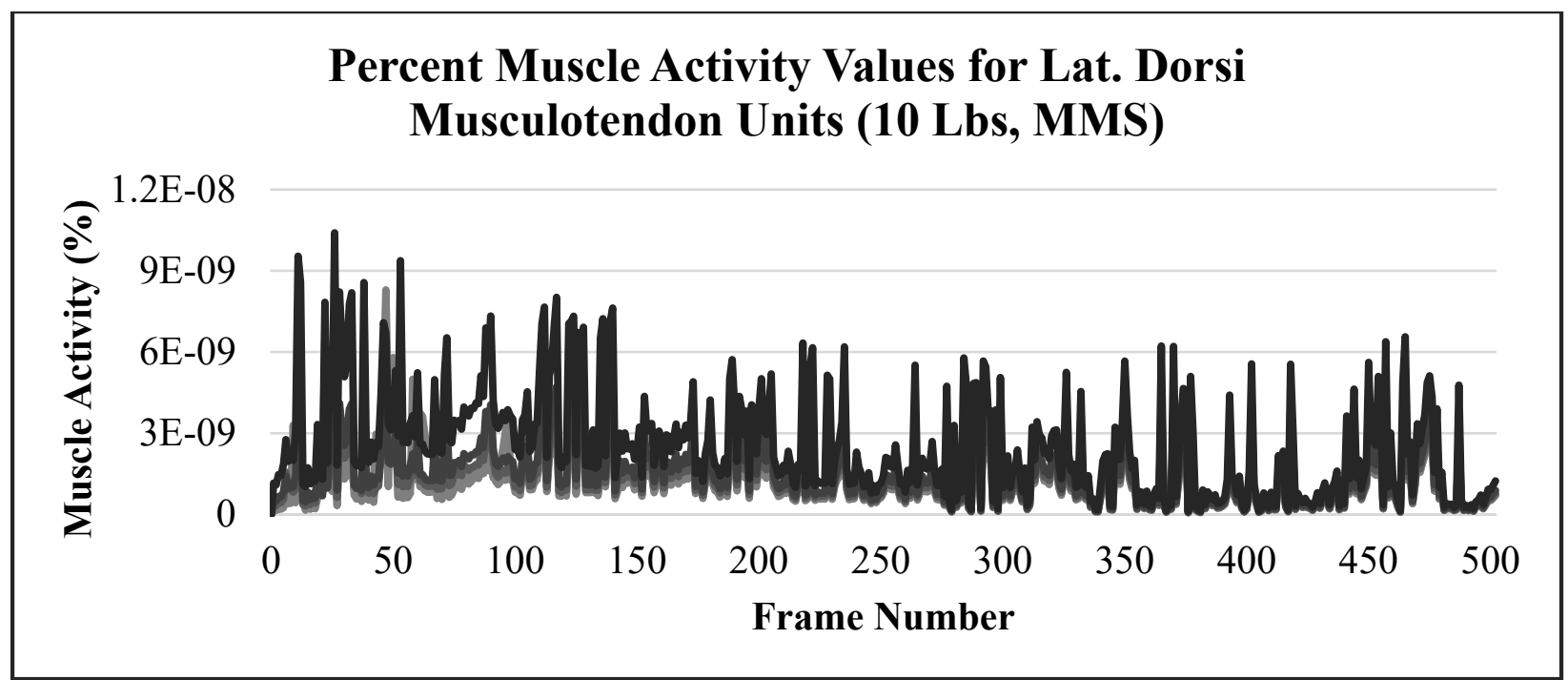

Figure 14. Percent Muscle Activity (\%MA) values for the five Latisimmus Dorsi musculotendon units from the 10-pound lifting task modeled with the MMS recruitment strategy during the dynamic portion of the task.

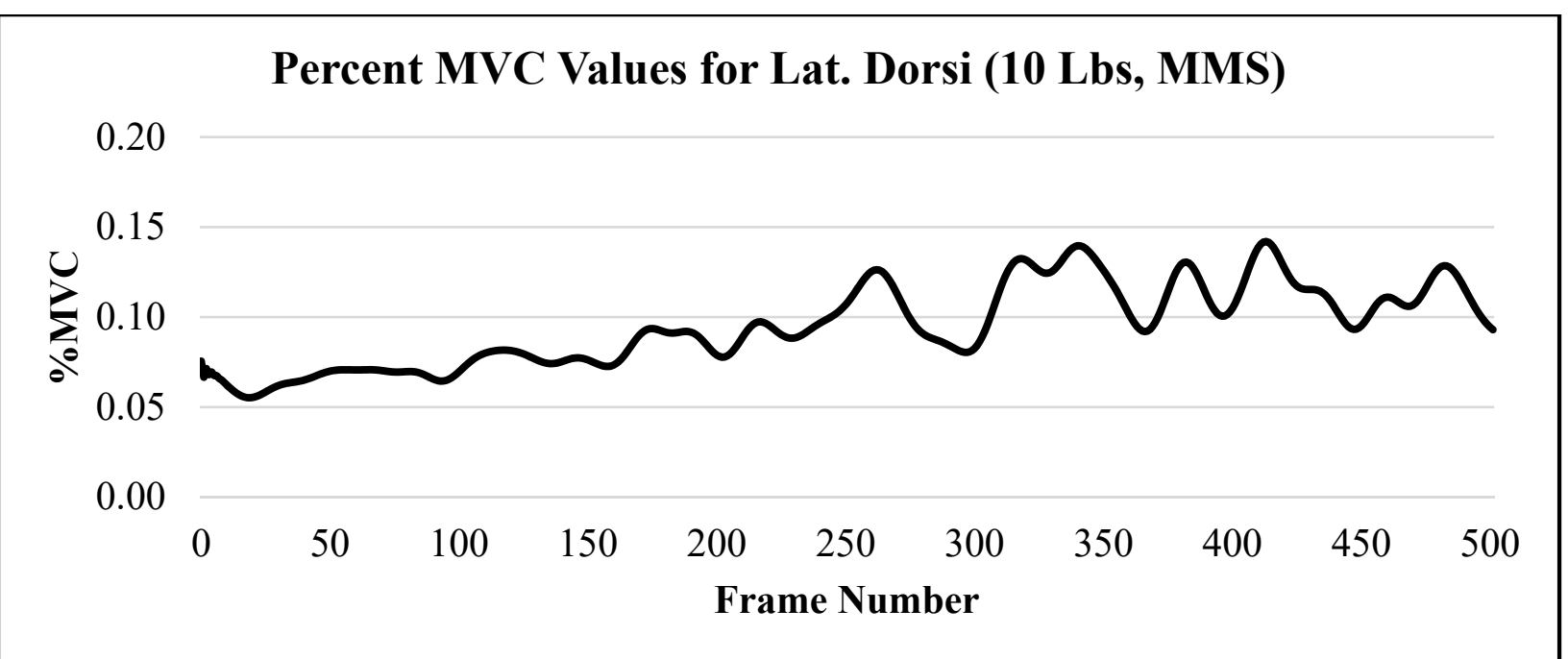

Figure 15. Percent MVC (\%MVC) values for the Latissimus Dorsi muscle from the 10-pound lifting task during the dynamic portion of the task. 
correlation value that Zee et al. considered acceptable. The correlation values for the remaining five muscles, the Anterior, Middle, and Posterior Deltoid, Infraspinatus, and Supraspinatus, were all above 0.80.

Generally, as the weight lifted increased, the correlation increased, as well (Figure 16). The exception was the MMS recruitment strategy, where the mean correlation for the 10 pound condition was lower than for the 5 pound condition, but still higher than the 2.5 pound condition. This is likely due to small amounts of noise in the data that have a much more pronounced effect on the correlation with smaller

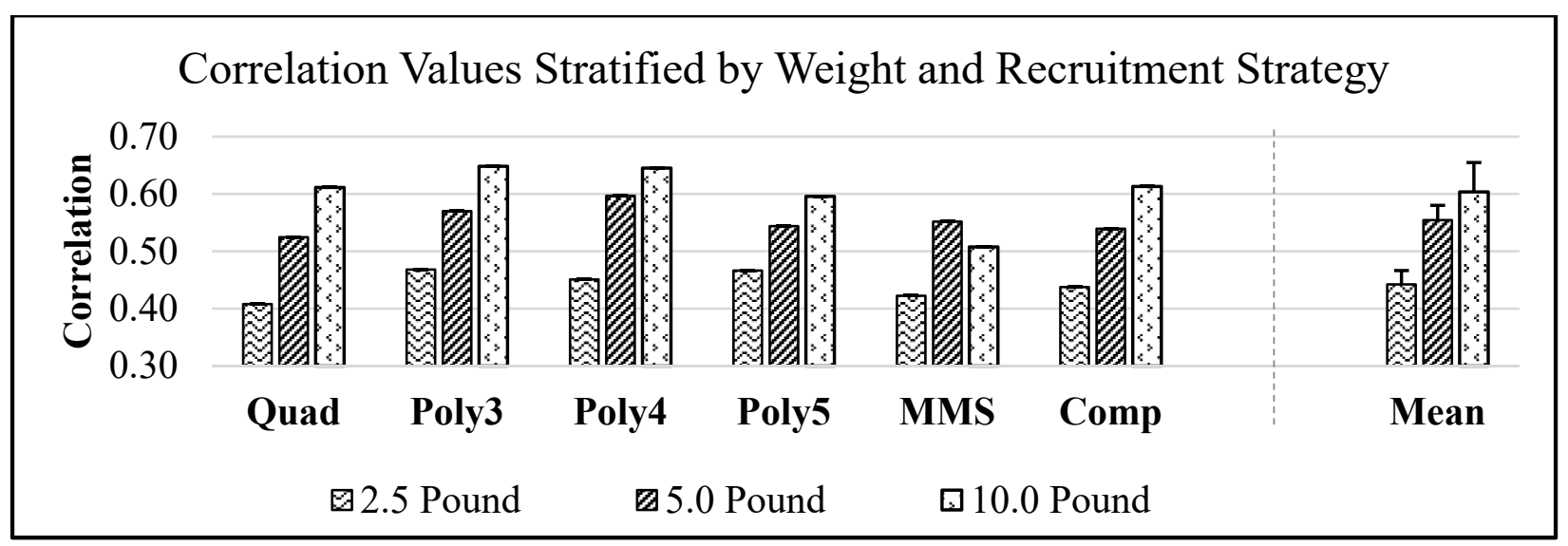

Figure 16. Correlation values stratified by weight and recruitment strategy, including the mean correlation for each weight condition and associated standard deviation bars, for the dynamic analysis.

$\% \mathrm{MVC}$ and \%MA values. The same general trend also existed when the data is stratified by muscle and weight, with the correlation values for each muscle generally increasing as the weight of the lift increased (Figure 17).

2.11.2 Static Analysis: Mean correlations for each recruitment strategy across all weights ranged from 0.7338 for the Composite strategy up to 0.8072 for the Min/Max strategy. Corresponding RMSE values ranged from 0.0235 for the Mix/Max Strategy up to 0.0258 for the Quadratic Strategy (Table 6). The MMS recruitment strategy showed the highest correlation values and the lowest RMSE values of the recruitment 


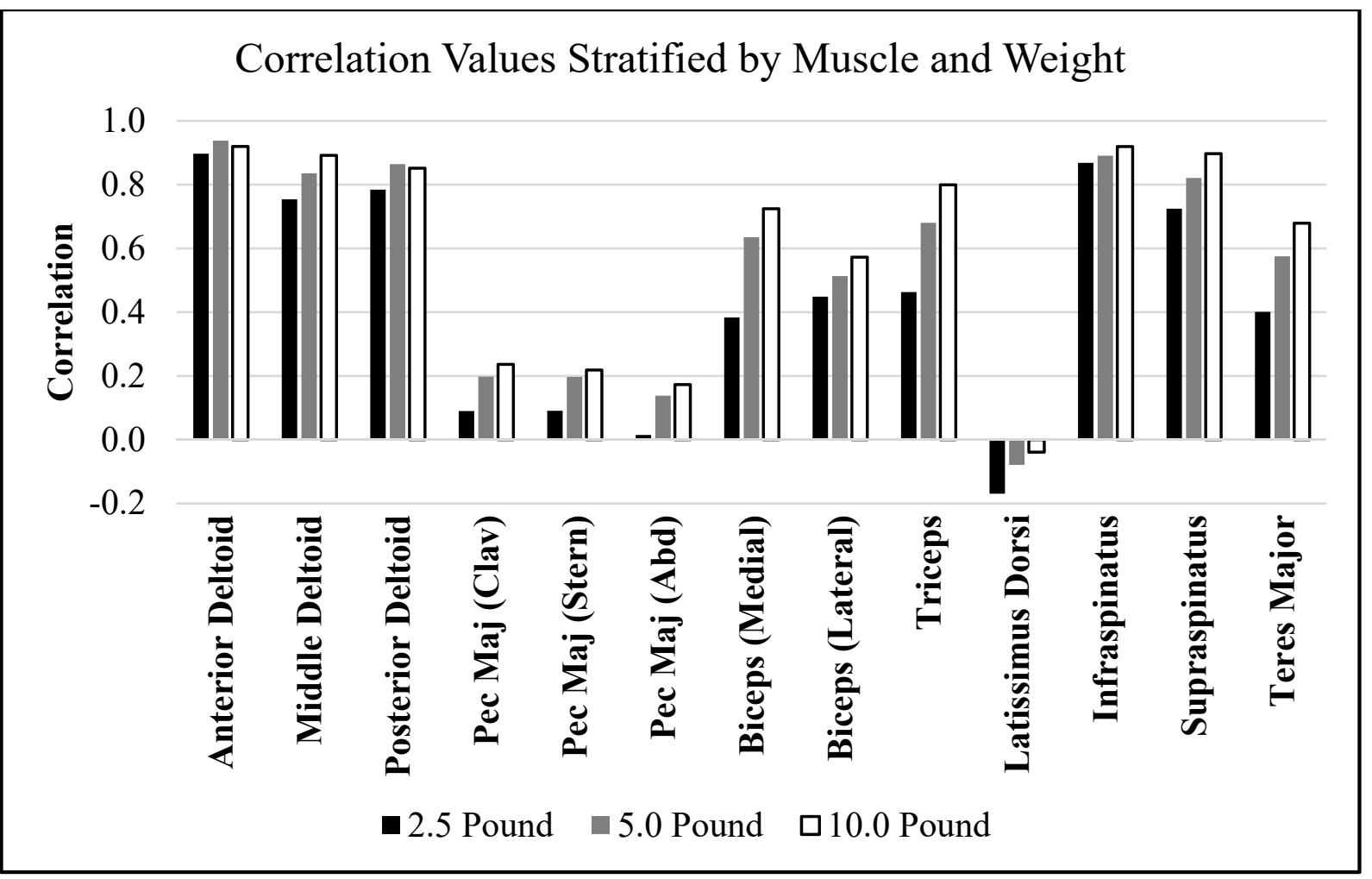

Figure 17. Correlation values stratified by muscle and weight for the dynamic analysis.

Table 6. Overall mean correlation values and mean RMSE values for each recruitment strategy for the static analysis.

\begin{tabular}{|l|r|r|}
\hline Recruitment Strategy & Correlation & RMSE (\%) \\
\hline Quadratic (Quad) & 0.7492 & 0.0258 \\
\hline 3rd-order Polynomial (Poly3) & 0.7408 & 0.0250 \\
\hline 4th-order Polynomial (Poly4) & 0.7558 & 0.0245 \\
\hline 5th-order Polynoimal (Poly5) & 0.7771 & 0.0242 \\
\hline Min/Max (MMS) & 0.8072 & 0.0235 \\
\hline Composite (Comp) & 0.7338 & 0.0255 \\
\hline
\end{tabular}




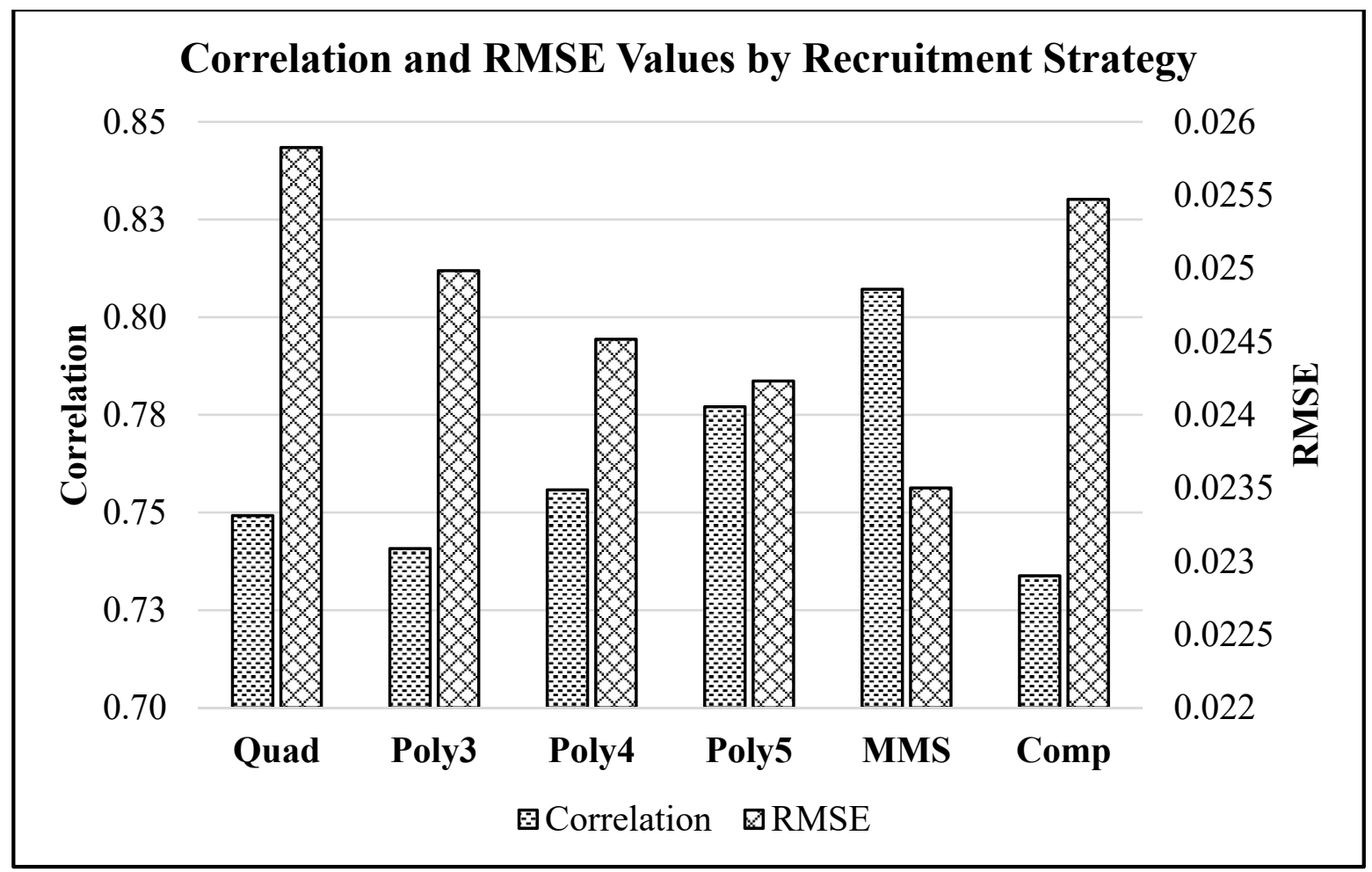

Figure 18. Mean correlation values and mean RMSE values found for each recruitment strategy for the static analysis.

strategies (Figure 18). The RMSE values in this case were very low, suggesting that the proportional contributions of each muscle remain relatively consistent across all recruitment strategies.

A much weaker relationship was observed for the static analysis compared to the dynamic task when data was stratified by weight. There was a general upward trend in the correlation values as the weight lifted increased for three of the recruitment strategies (Poly3, Poly4, and Comp), while the remaining three recruitment strategies showed no trend (Figure 19). Also, while the mean did show an upward trend, when considering the standard deviations, the trend is unconvincing. When the correlations for MMS, the recruitment strategy with the highest correlations, were stratified by weight, the correlations remained consistent as weight increased, suggesting that the model is reliable regardless of the level of exertion. 


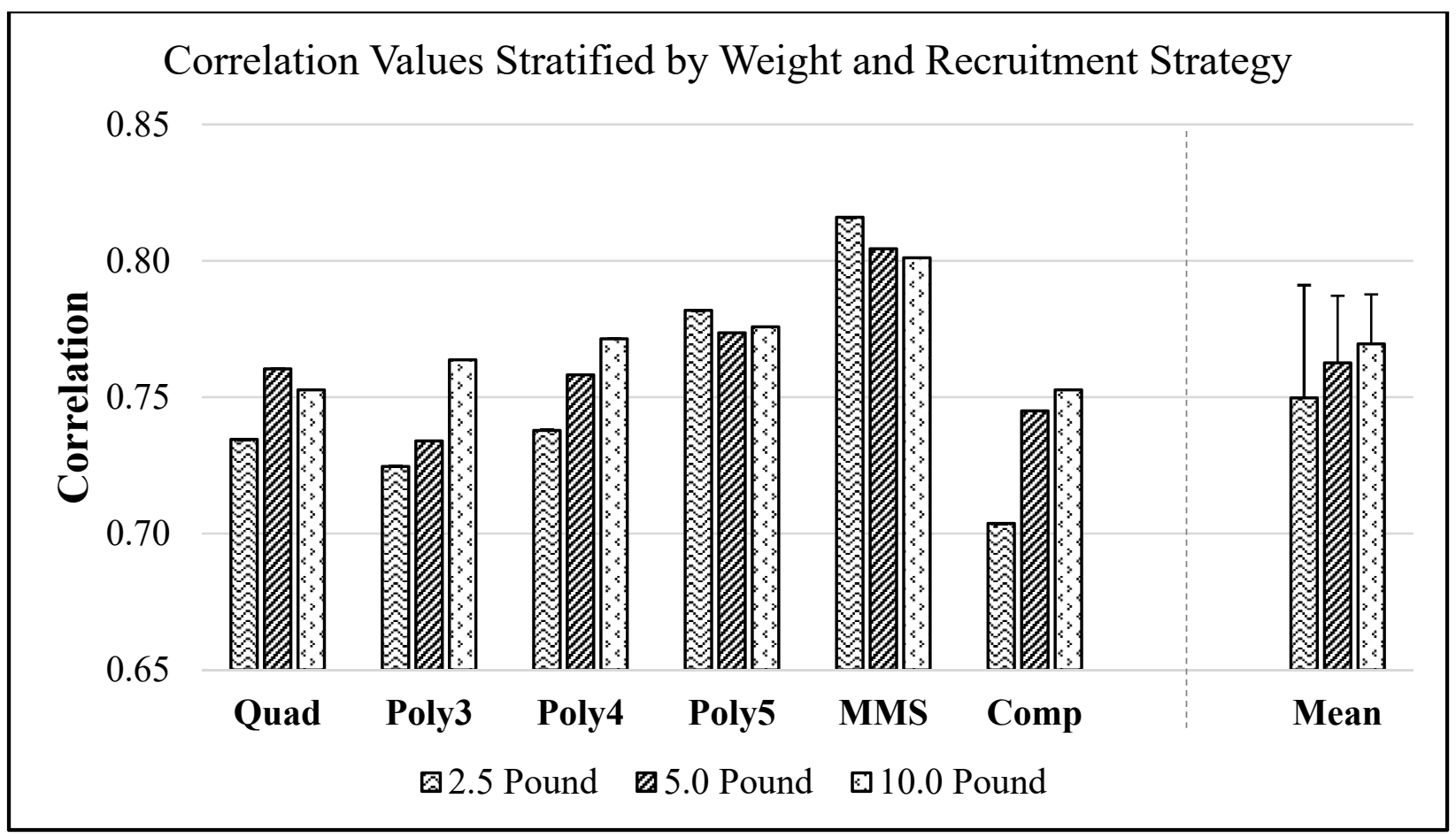

Figure 19. Correlation values stratified by weight and recruitment strategy for the static analysis.

\subsection{Discussion}

Electromyography data for 13 muscles was compared to corresponding muscle activity value output from the musculoskeletal model. Based on the analysis, the most accurate muscle recruitment strategy for dynamic tasks of the shoulder was the $4^{\text {th }}$-order Polynomial Recruitment. On the other hand, the results for the static portion of the tasks found the Min/Max Recruitment to be the most accurate recruitment strategy. 


\section{SPECIFIC AIM \#2: COMPOSITE INDEX AND PREDICTIVE EQUATION DEVELOPMENT}

\subsection{Background}

The shoulder is comprised of four bones, the humerus, clavicle, sternum, and scapula. These bones form the three joints of the shoulder, the sternoclavicular joint, acromioclavicular joint, and Glenohumeral joint (Figure 20). The sternoclavicular (SC) joint is at the junction of the lateral aspect of the manubrium of the sternum and the medial end of the clavicle. The SC joint allows motion of the clavicle in three planes, but has no musculature acting at the joint. The acromioclavicular (AC) joint is at the junction of the acromion process of the scapula and the lateral end of the clavicle. The AC joint acts as a pivot point, allowing the arm to be raised above the head. Like the SC joint, there is no musculature acting on the AC joint. The Glenohumeral (GH) joint, the junction of the glenoid fossa on the lateral aspect of the scapula and the humeral head, is the major load-bearing joint of the shoulder. The contact surface between the glenoid fossa and humeral head, on average, ranged from 2.51 to $5.07 \mathrm{~cm}^{2}$, depending on posture (Soslowsky et al., 1992) and only $25-30 \%$ of the humeral head is in contact with the glenoid fossa at any given time (Terry and Chopp, 2000). Due in part to this anatomy, the GH joint is the most mobile joint in the body (Terry and Chopp 2000), with a range of motion covering 65\% of a sphere (Engin and Chen, 1986). However, the mobility of the shoulder comes with a sacrifice in inherent stability.

Due to the small contact area between the humeral head and glenoid, the stability of the shoulder relies on ligaments, the shoulder muscles, and the glenoid labrum. The ligaments crossing the Glenohumeral joint, the superior, middle, and inferior Glenohumeral ligaments, assist in stabilization at the end ranges of

motion, but at near neutral positions the ligaments are lax and provide little stability. The rotator cuff muscles, however, provide the majority of the stability to the shoulder throughout the entire range of motion due to the concavity-compression mechanism. The resultant force caused by action of the rotator cuff 
muscles is directed inward, compressing the humeral head against the glenoid fossa. In a study utilizing 10 fresh-frozen cadaveric shoulders, Lippitt et al. (1993) applied a 50N and, subsequently, 100N compressive

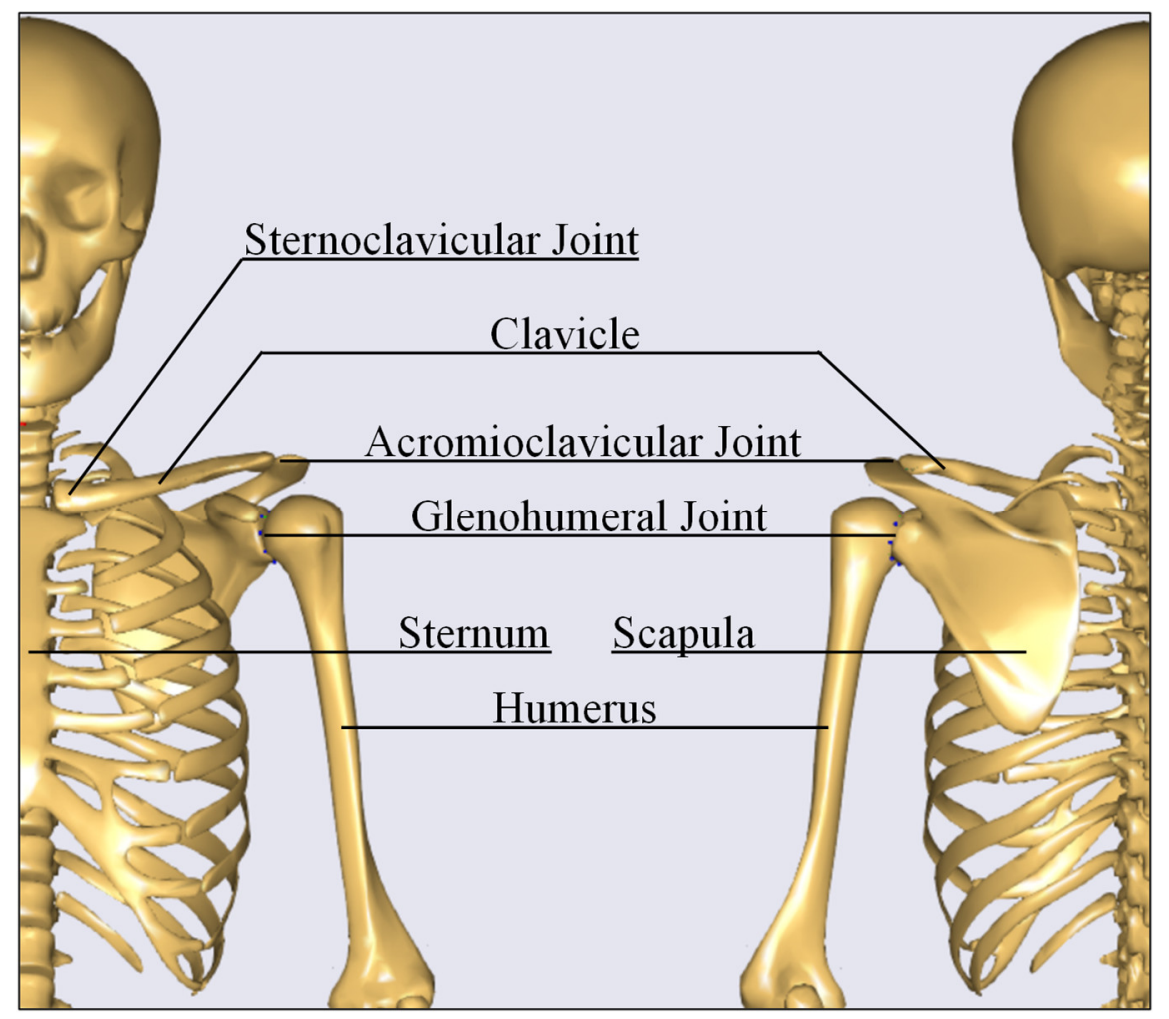

Figure 20. Bones and joints of the shoulder joint

force on the humeral head. At each compression level, a translational force was applied and increased until dislocation occurred. They performed this test with translational forces from 8 directions $\left(45^{\circ}\right.$ intervals around the joint). The results of the research showed that resistance of the shoulder to translational forces increases as compression of the humeral head into the glenoid increases. The glenoid labrum, a fibrocartilage "rim" around the edge of the glenoid, assists in resisting dislocation and improving stability by increasing the depth of the glenoid. In a study of cadaveric shoulders, Halder et al. (2001a) found a reduction in stability (i.e. reduced resistance to dislocation from translational forces) of approximately $10 \%$ when the labrum was excised. 
While the rotator cuff muscles and other muscles responsible for motion of the shoulder generally work together to stabilize the shoulder, not all muscles in the region provide stabilizing forces at the Glenohumeral joint. Labriola et al. (2005) used a musculoskeletal model of the shoulder to determine how the reaction force at the Glenohumeral joint, and therefore the stability of the shoulder, was affected as magnitudes of force of the shoulder muscles were increased by $25 \%$ and $50 \%$. The results of their analysis showed that activation of the Supraspinatus, Infraspinatus, and Teres Minor led to increased stability at the shoulder, while activation of the Deltoid or Pectoralis Major caused a decrease in stability. Changing the magnitude of force on the Teres Major, Subscapularis, and Latissimus Dorsi had no effect on stability. However, there is some evidence that it is not so straightforward. Lee et al. (2002) found that activation of the Deltoid in the scapular plane increased stability, while activation in the coronal plane caused a decrease in stability. This suggests that posture could alter the line of action and influence the effect on stability of each muscle. On the other hand, the role of the Biceps Brachii in shoulder stability seems much more straightforward. Itoi et al. (1993) replaced the long and short heads of the Biceps Brachii of 13 cadaver shoulders with spring devices. Forces of $0 \mathrm{~kg}, 1.5 \mathrm{~kg}$, and $3 \mathrm{~kg}$ were exerted anteriorly at the Glenohumeral joint with the humerus at $60^{\circ}, 90^{\circ}$, and $120^{\circ}$ abduction with the articular capsule in three different conditions (intact, vented, and damaged by Bankert Lesion). Both heads of the Biceps Brachii were found to stabilize the shoulder at $60^{\circ}$ and $90^{\circ}$ abduction in healthy shoulders. Rodosky et al. (1994) used a musculoskeletal model of the shoulder to determine the effect that the long head of the Biceps Brachii has on shoulder stability in overhead postures and found that the long head of the Biceps Brachii improved anterior stability by resisting torsional forces at the Glenohumeral joint during abduction and external rotation.

\subsection{Objectives}

Objective 1: Develop a Composite Index that relies on the Glenohumeral force outputs in the model to estimate shoulder strain. This index was derived based on the correlation relationship of electromyography and ratings of perceived exertion data with the composite index that is based on 
biomechanical and physiological properties of the shoulder, i.e. how the shoulder moves and the modeled forces resulting from that movement.

Objective 2: Develop a predictive equation and heatmaps that can be used to guide load placement to reduce load on the shoulder when developing material handling tasks. Regression methods were used to develop an equation that uses distances from the sternal notch as input to estimate the strain at the shoulder.

\subsection{Approach}

Participants performed a static lifting task at set distance intervals in three dimensions within the working range of the right arm. A 15 pound $(\sim 70 \mathrm{~N})$ weight was suspended at each point, and the participant lifted the weight slightly and held it for 5 seconds. This weight was selected because it is light enough that participants should be able to lift it throughout the working range of the arm, yet heavy enough to significantly activate the muscles. After each task, the participant was asked to rate their exertion level on a 10-point scale. Motion capture and electromyography data was collected during the experimental trials. Motion capture data was used to drive the model in AnyBody to obtain the reaction forces at the Glenohumeral joint throughout the task. A Composite Index with a high correlation with the rating of perceived exertion and electromyography data and that utilized physiologically-based, model-derived strain components was developed. A predictive equation was developed to estimate the Composite Index values based on the three-dimensional distance (forward, left/right, upward/downward) to the load using the sternal notch as a reference point. With consideration given to the subjective ratings of perceived exertion, the range of strain values, and the Glenohumeral force characteristics, risk cut-off criteria with three range categories were developed (Figure 21). 


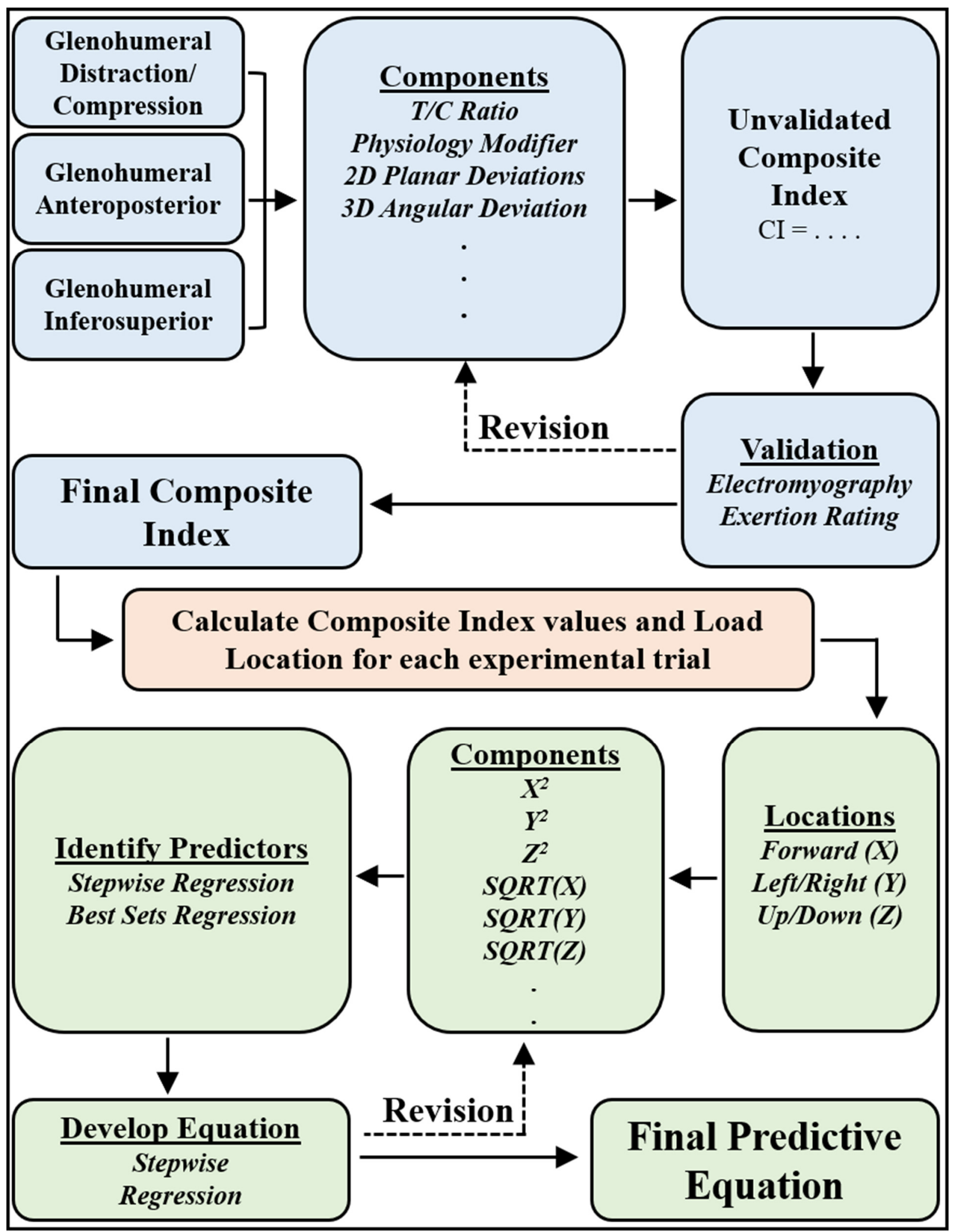

Figure 21. Progression for Specific Aim 2. 


\subsection{Participants}

A convenience sample of five different, healthy male participants were recruited with mean $( \pm \mathrm{sd})$ age, height, and weight of $26.2( \pm 5.8)$ years, $1.76( \pm 0.04)$ meters, and $68.04( \pm 8.49) \mathrm{kg}$, respectively. The participant heights and weights are provided in Table 7. The height and weight data were utilized in the anthropometrical scaling of the musculoskeletal model. For inclusion in the study, potential participants were male, aged 18-40 years old, and reported no musculoskeletal disorders that would affect their ability to perform the tasks or that could affect the results. All participants confirmed to being right-hand dominant. Male participants were recruited for consistency with the first specific aim and due to the same conditions that constrained specific aim one to male participants.

Table 7. Heights and weights of each participant in Specific Aim 2.

\begin{tabular}{lccccc}
\hline Participant: & $\mathbf{1}$ & $\mathbf{2}$ & $\mathbf{3}$ & $\mathbf{4}$ & $\mathbf{5}$ \\
\hline Height $(\mathrm{m})$ & 1.78 & 1.70 & 1.75 & 1.80 & 1.75 \\
Weight $(\mathrm{kg})$ & 81.65 & 58.97 & 68.04 & 68.04 & 63.50 \\
\hline
\end{tabular}

While there are no hard rules for sample size determination for regression analysis, Green et al. (1991) found evidence of support for a sample size following Equation 7.

$$
\begin{aligned}
& \begin{array}{l}
N>50+8 m, \\
\text { where; } \mathrm{N}=\text { sample size; } \\
\qquad \mathrm{m}=\text { Number of predictors. }
\end{array}
\end{aligned}
$$

Accounting for potential losses during modeling, at least 70 samples per participant are expected. With 5 participants, data from at least 350 trials across all 5 participants should be available. This would allow up to 12 predictors. However, Green et al. method assumes independent samples, while this research was performed with numerous samples per participant, thus, the trials are not all independent. Regardless, this research is exploratory in nature and resources were limited. 


\subsection{Equipment}

Aside from the AnyBody modeling system software, the following Equipment will be used in this stage of the research.

3.5.1 Data Acquisition Hardware: Same as Specific Aim \#1 (See Section 2.5.1)

3.5.2 Hydraulic Scissor Lift (Vestil EHLT-4872-3-43, Vestil Mfg., Angola, IN, USA): An electric scissor lift with platform dimensions of 48 inches by 72 inches and weight capacity of 3000 pounds was used to support and provide vertical adjustability to a custom weight support.

3.5.3 Custom Weight Support and Floor Grid: A custom built wooden structure was securely attached to the front of the hydraulic scissor lift (Figure 22). The structure was used to support a hanging weight. A rope supporting the weight was marked in 10 inch intervals such that the weight could be easily adjusted to the heights of interest $(-20,-10,0,10$, and 20 inches from the sternal notch).

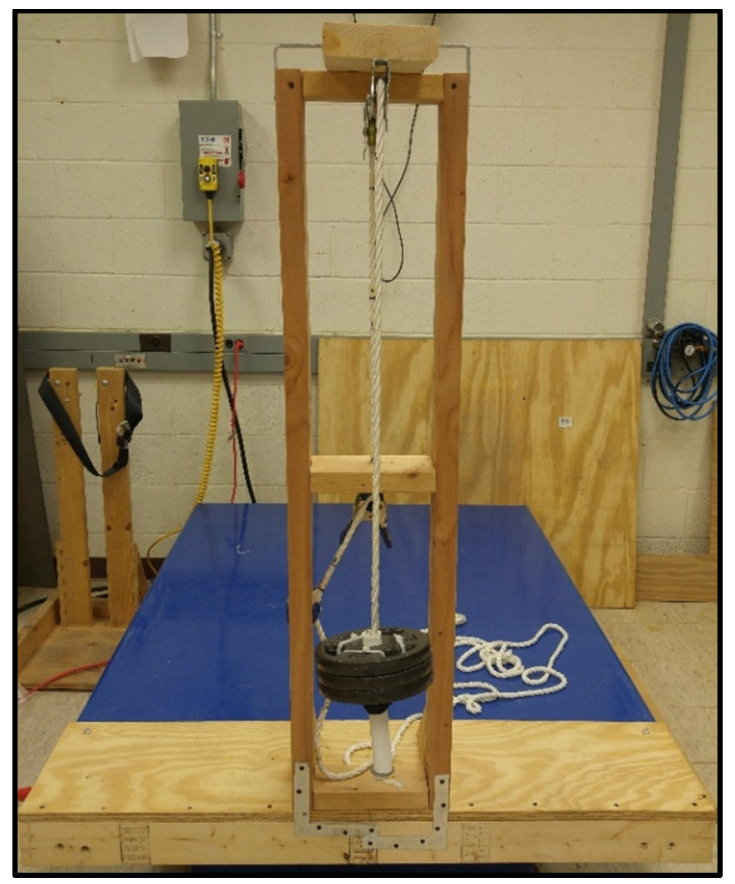

Figure 22. Custom-built wooden structure to support hanging weight. 
Several pieces of foamboard were attached to create a foamboard platform that is 70 inches by 40 inches. The gridlines left-to-right were labeled $32 \mathrm{R}, 24 \mathrm{R}, 16 \mathrm{R}, 8 \mathrm{R}, 0,8 \mathrm{~L}, 16 \mathrm{~L}$, and $24 \mathrm{~L}$. The gridlines forward-to-back were labeled $0,8,16$, and 24 (Figure 23). Intervals of 6 inches and 10 inches were considered, but the 6-inch intervals increased the number of trials significantly, while the 10-inch intervals did not provide the desired resolution. Intervals of 8 inches provided a good compromise between number of trials each participant must perform and the resolution of the data. After drawing the gridlines, the foamboard was sprayed with several coats of a clear rubberized coating for protection, longevity, and easy clean-up.

\subsection{Experimental Design}

Each participant performed the lifting tasks while standing at every grid point that is within reach of the hanging weight at each of the five heights, and subsequently provided a rating of the exertion. Each lifting task was modeled in AnyBody Modeling system to calculate internal forces on the Glenohumeral joint. Dependent factors were the model outputs and ratings of perceived exertion. Independent factor was the location of the weight with respect to the sternal notch. A rest period of at least 1 minute was provided between lifts. Exertions were performed once at each location.

\subsection{Participant Consent and Preparation}

Upon arrival, participants were introduced to the equipment and study procedures and consent was obtained with a form approved by the local Institutional Review Board (Appendix F). Participants then completed the Physical Activity Readiness Questionnaire (PAR-Q) to screen for cardiorespiratory fitness (Appendix C). Answering "No" to any question would have disqualified the participant from continuing. Motion capture marker-set and placement details can be found in Section 2.7. Electromyography sensors were placed superficially to the same 13 muscles measured in Specific Aim 1 (Section 2.7) and described in Table 2, after shaving and cleaning the area. 

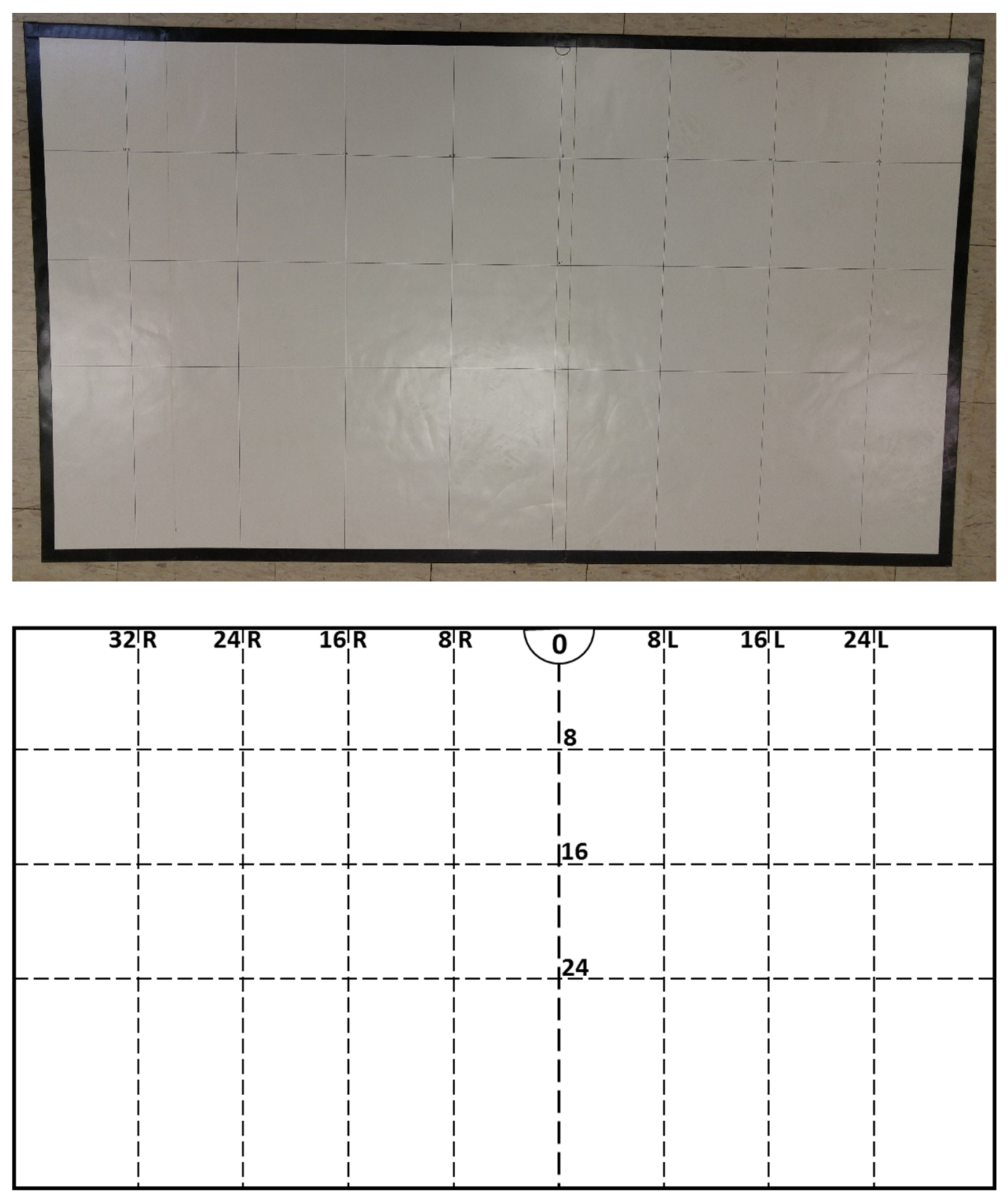

Figure 23. Top) Actual foamboard grid used for the experimental tasks. Bottom) Digital mock-up of foamboard grid layout for easier visualization.

The foamboard grid was placed on the floor such that, when the participant stood with their toes lined up with the ' 0 ' forward line and straddling the ' 0 ' left/right mark, the hanging weight was directly in line with the CLAV marker at the sternal notch. The height of the hanging weight in the custom weight 
support was adjusted with the rope at the ' 0 ' mark. The electric hydraulic scissor lift was adjusted such that the weight, while at the ' 0 ' mark, is at the same height as the CLAV marker on the participant's sternal notch.

\subsection{Maximum Voluntary Contractions}

The participant performed the seven maximum voluntary contraction exertions following the same procedures detailed in Section 2.8. Each MVC exertion was performed three times with a two-minute rest period provided between each repetition.

\subsection{Experimental Tasks}

The participant stood upright with their arms down to their side at the instructed location on the floor grid. When signaled to begin, while keeping the trunk still, the participant grasped the weight with their right hand and gently lifted it just enough to support the mass of the weight and remove tension on the rope it was suspended from. Participants were instructed to remain as still as possible while supporting the weight. After supporting the weight for approximately 6 seconds, the participant gently lowered and released the weight. After releasing the weight, the participant was asked to rate their level of exertion on a 10-point Borg Scale (Appendix G). This procedure was followed for each of the locations that were within reach of the participant while maintaining a stable, upright trunk, resulting in approximately 80-90 trials per participant, depending on their height and reach. Trial order was determined by randomizing the order of the 5 heights, then randomizing the locations within that height. This was done instead of randomizing over all trials to avoid having to adjust the height of the weight between each trial. A one-minute rest period was provided between trials.

\subsection{Data Processing}

3.10.1 Musculoskeletal Modeling: Each experimental trial was processed in Vicon Nexus following the same procedure outlined in Section 2.10.1. While processing each trial in Nexus, a 5 second period in which the participant was supporting the weight was identified and start and end points were identified and 
recorded. The marker data was exported from Vicon Nexus in C3D file format and modeled in AnyBody Modeling Software using the same model described in Section 2.10.2 with two minor modifications. First, the model was modified to output the reaction forces at the Glenohumeral joint. Second, the model was only used with the MMS muscle recruitment strategy. Per the results from Specific Aim 1, the MMS muscle recruitment strategy was determined to provide most accurate results for static-type exertions. The focus in this section of the research was on the span of time during each trial that the participant was supporting the weight, which was a static exertion. Reaction forces at the Glenohumeral joint were output in three orthogonal dimensions: compression/distraction (CD), anteroposterior (AP), and inferosuperior (IS). Compression/distraction force is the magnitude of the force perpendicularly inward or outward from the Glenohumeral joint. Anteroposterior and inferosuperior forces, considered translational forces, are the magnitude of the force in the forward/backward and upward/downward directions, respectively. Adding these three vectors gave the magnitude and direction of the resultant force at each frame.

3.10.2 Electromyography Data: Electromyography data was used to evaluate and validate the potential composite indices and predictive equations. To consolidate the EMG data from each experimental trial into one value, an EMG summation was calculated. First, maximum voluntary contraction values were calculated using methods described in Section 2.10.3. To calculate the EMG summation value, the data was first demeaned, rectified, and filtered as described in Section 2.10.3. The five seconds of each trial identified in Section 3.10.1 were extracted. For each of the 13 muscles, each data point was divided by the MVC of that muscle for that participant to normalize the data into \%MVC values. For each trial, the mean of the $\% \mathrm{MVC}$ values over the 5 seconds period for each trial were calculated and added across all 13 muscles to provide a single EMG summation value.

\subsection{Results}


As discussed in Section 3.9, data points varied per participant due to differences in reach distance. Additionally, other trials were lost in analysis due to either 1) hidden/obstructed motion capture markers or 2) failure during the musculoskeletal modeling process in AnyBody Modeling System. It was not unusual for the markers on the hand to be obstructed by the equipment (weight support and, for the lower heights especially, the scissor lift) and the participant's torso. Gap-filling techniques discussed in 3.1.10.1 were used for small gaps, but when a high level of accuracy couldn't be guaranteed, the trial was discarded. Failures during the musculoskeletal modeling process occurred in both the kinematic determination and inverse dynamics phases.

Failures in the kinematic determination phase occurred when the markers could not be "matched up" within the tolerance specified in the model. Model scaling, marker placement accuracy, marker movement during data collection, and gap-filling accuracy contributed to success of the kinematic determination phase for each trial. Failures in the inverse dynamics phase occurred when a solution to the muscle recruitment couldn't be found based on the criteria of the model. The root of these failures was primarily inaccuracy in the kinematic determination phase, resulting in the following modes of failure in the model:

1. Overloaded Muscle Configuration: The amount of force that can be exerted by the musculotendon units is specified within the model and scales based on the stature and weight of the participant. This was left at the default value. If there is an inaccuracy in the kinematic determination, this can lead to higher force requirements of the musculotendon units.

2. Surface Penetration: This occurs when the musculotendon units in the model penetrate the surface of another muscle or bone. Since muscles are disabled during kinematic determination, the model will succeed in that phase and fail when muscles are included for the inverse dynamic analysis.

3. Maximum Iterations: This failure occurs when a solution is not found after the specified number of attempted iterations. This value was left at the default of 20,000. 
In total, data from 443 experimental trials was collected with 352 (79.4\%) successfully continuing through the motion capture data processing and modeling in AnyBody Modeling System. A per-participant breakdown is provided in Table 8 .

Table 8. Per-participant breakdown of number of collected trials, losses during motion capture, losses during modeling, and successful trials used in final analysis.

\begin{tabular}{lccccc|c}
\hline Participant: & $\mathbf{1}$ & $\mathbf{2}$ & $\mathbf{3}$ & $\mathbf{4}$ & $\mathbf{5}$ & Total \\
\hline Collected Trials & 91 & 85 & 87 & 93 & 87 & $443(100 \%)$ \\
MoCap Losses & 12 & 12 & 5 & 10 & 6 & $45(10.2 \%)$ \\
Modeling Losses & 11 & 17 & 9 & 5 & 4 & $46(10.4 \%)$ \\
Successful Trials & 68 & 56 & 73 & 78 & 77 & $352(79.4 \%)$ \\
\hline
\end{tabular}

\subsection{Composite Index Development}

Throughout the development of the composite index and predictive equations, correlation of the results with both perceived exertion rating reported by the participant and EMG summation, calculated as discussed above, were used to evaluate performance. Pearson's correlation (Equation 4, where: $x_{i}=$ EMG summation value for $i$ th data point, and $y_{i}=$ composite index value for $i$ th data point) was used between the potential composite index values and the EMG summation. However, due to the non-continuous nature of the perceived exertion rating, Spearman's rank-order correlation (Equation 6, where $d_{i}=$ difference in rank of the $i$ th data point between perceived exertion rating data and composite index value data) was used between the potential composite index values and the exertion ratings.

Composite index development began with correlation analysis of several potential components that are based on the concepts of the concavity-compression mechanism. The shoulder is, conceptually, most stable in the absence of translational forces, where no force is pushing the humeral head away from the glenoid fossa and the resultant force vector is directed medially (stable axis). As translational forces increase without an accompanying increase in compressive forces, the resultant force vector at the Glenohumeral joint deviates from this stable axis and risk of shoulder injury increases. The following components were tested: 
1. Magnitudes of Resultant Force Vector and Components: Magnitudes of the vector components are given in the software, where:

$F_{z}$ is the anterosuperior force,

$F_{y}$ is the inferosuperior force, and

$F_{z}$ is the compression/distraction force.

The magnitude of the resultant force vector is calculated as the root of the sum of squares of the individual vector components (Equation 8)

$$
M=\sqrt{F_{x}^{2}+F_{y}^{2}+F_{z}^{2}}
$$

2. Normalized Two Dimensional Planar Angles $(\alpha, \beta$, and $\gamma)$ : The resultant force vector at the glenohumeral joint was mirrored onto two-dimensional anatomical planes to calculate the angle between the vector and each axis. $\alpha, \beta$, and $\gamma$, the angles the resultant forces form in the sagittal, frontal, and transverse planes from the axes (Figure 24), respectively, normalized to $180^{\circ}$, which is the highest angle possible. $\alpha, \beta$, and $\gamma$ were calculated using Equation 9, Equation 10, and Equation 11, respectively.

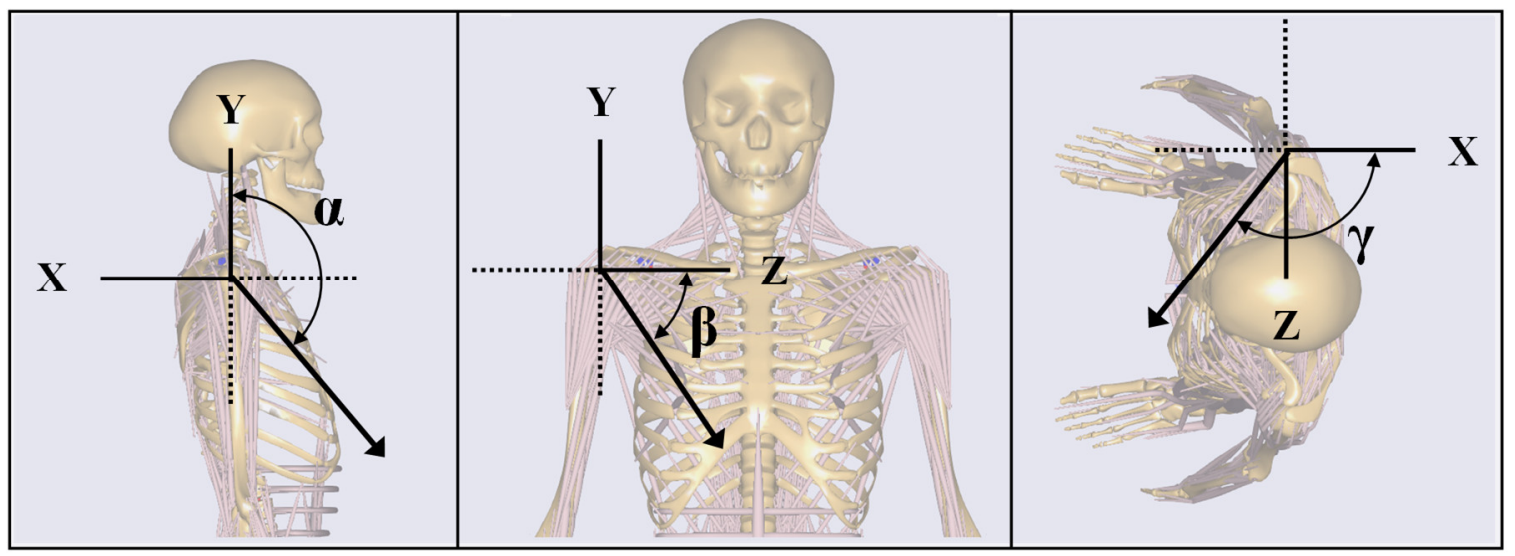

Figure 24. Two-dimensional angular deviations. In this figure, $\alpha, \beta$, and $\gamma$ are the angles that were normalized by $180^{\circ}$ using Equations 7, 8, and 9 . 


$$
\alpha=\frac{\tan ^{-1}\left(\frac{F_{y}}{F_{x}}\right)}{180}
$$

where:

$F_{x}$ is the anterosuperior force, and

$F_{y}$ is the inferosuperior force.

$$
\beta=\frac{\tan ^{-1}\left(\frac{F_{y}}{F_{z}}\right)}{180}
$$

where:

$F_{y}$ is the inferosuperior force, and

$F_{z}$ is the compression/distraction force.

$$
\gamma=\frac{\tan ^{-1}\left(\frac{F_{\chi}}{F_{z}}\right)}{180}
$$

where:

$$
\begin{aligned}
& F_{x} \text { is the anteriosuperior force, and } \\
& F_{z} \text { is the compression/distraction force. }
\end{aligned}
$$

3. Normalized Three-Dimensional Angular Deviation $(\mu)$ : The angle formed between the resultant force vectors and a unit vector along the $\{-Z\}$ axis (distraction direction) was calculated then normalized to $180^{\circ}$ (Equation 10):

$\mu=\frac{\cos ^{-1}\left(\frac{-F_{z}}{\sqrt{F_{x}^{2}+F_{y}^{2}+F_{z}^{2}}}\right)}{180}$

where: 


$$
\begin{aligned}
& F_{z} \text { is the anterosuperior force, } \\
& F_{y} \text { is the inferosuperior force, and } \\
& F_{z} \text { is the compression/distraction force. }
\end{aligned}
$$

4. Translational/Compressive Ratio (TC): The ratio between the translational forces (i.e., the resultant of the anteroposterior and inferosuperior forces) and the compression/distraction force (Equation 11).

$$
T C=\frac{\sqrt{F_{x}^{2}+F_{y}^{2}}}{F_{z}^{2}}
$$

where:

$$
\begin{aligned}
& F_{z} \text { is the anterosuperior force, } \\
& F_{y} \text { is the inferosuperior force, and } \\
& F_{z} \text { is the compression/distraction force. }
\end{aligned}
$$

The correlation values found between each of these components and the exertion ratings and EMG summations are shown in Table 9.

Table 9. Correlations coefficients and p-values resulting from correlation analysis between each of the potential components and the EMG Summation and perceived exertion rating.

\begin{tabular}{c|cc|cc}
\hline \multirow{2}{*}{ Component } & \multicolumn{2}{c}{ EMG Summation } & \multicolumn{2}{c}{ Exertion Rating } \\
\cline { 2 - 4 } & Pearson $r$ & $p$-Value & 0.4586 & $p$-Value \\
\hline$F_{x}$ & 0.5831 & 0.0000 & 0.4643 & 0.0000 \\
$F_{y}$ & 0.5961 & 0.0000 & -0.3689 & 0.0000 \\
$F_{z}$ & -0.5853 & 0.0000 & 0.4037 & 0.0000 \\
Magnitude & 0.6028 & 0.0000 & -0.2470 & 0.0000 \\
$\alpha$ & -0.3972 & 0.0000 & 0.0905 & 0.0000 \\
$B$ & 0.2549 & 0.0000 & -0.3096 & 0.0895 \\
$\gamma$ & -0.3259 & 0.0000 & -0.0190 & 0.0000 \\
$\mu$ & -0.1656 & 0.0018 & 0.7216 \\
TC Ratio & 0.1656 & 0.0018 & 0.0215 & 0.6874 \\
\hline
\end{tabular}


Generally, components related to the magnitude of the resultant force vector and its components showed the highest correlation with the EMG Summation $(r=0.5831$ to $r=0.6028)$ and exertion rating $(r$ $=0.3689$ to $\mathrm{r}=0.4643$ ). However, the strength of correlation was mediocre across all components.

In previous research that aimed to estimate shoulder strain based on model outputs from AnyBody Modeling System, Chowdhury et al. (2018) developed and validated a strain index (Equation 12) based on the concept of the concavity-compression mechanism. The equation is the sum of two primary "components", each following a format of "Normalized Angle*Normalized Magnitude". The components are a product of 1) the normalized magnitudes of the Glenohumeral resultant force vector when projected onto the frontal plane for the first component (similar to Figure $25, \beta$ ) and the sagittal plane for the second component (similar to Figure 25, $\mathrm{\gamma}$ ) and 2) the normalized angle between these two vectors and a vector drawn horizontally laterally from the Glenohumeral joint. However, this strain index calculation required an MVC reference to calculate $R_{f-M V C}$ (Equation 13) and $R_{t-M V C}$ (Equation 14).

$I_{1}=\left[\left(\frac{\sqrt{F_{y}^{2}+F_{z}^{2}}}{R_{f-M V C}} * \frac{\tan ^{-1}\left(\frac{F_{y}}{F_{z}}\right)}{45}\right)+\left(\frac{\sqrt{F_{\chi}^{2}+F_{z}^{2}}}{R_{t-M V C}} * \frac{\tan ^{-1}\left(\frac{F_{\chi}}{F_{z}}\right)}{45}\right)\right] * 100$

where:

$F_{x}$ is anterioposterior force,

$F_{y}$ is inferosuperior force, and

$F_{Z}$ is compression/distraction force.

$R_{f-M V C}=\sqrt{F_{y}^{2}+F_{z}^{2}}$

(Equation 13)

where:

$F_{y}$ is inferosuperior force from MVC reference trial, and

$F_{Z}$ is compression/distraction force from MVC reference trial.

$R_{t-M V C}=\sqrt{F_{x}^{2}+F_{z}^{2}}$

(Equation 14) 
where:

$F_{x}$ is anterioposterior force from MVC reference trial, and

$F_{Z}$ is compression/distraction force from MVC reference trial.

The MVC reference was performed by selecting the trial from each participant which best met the following criteria: 1) arm reached farthest forward from the body, 2) arm as close to the midline (sagittal plane) as possible, and 3) arm as close to shoulder level as possible. The selected trial was modeled again, except the downward force was increased in 1 Newton increments until the model failed due to an overloaded muscle configuration. The resulting Glenohumeral forces were used in calculations of $R_{f-M V C}$ and $R_{t-M V C}$.

When this strain index was calculated for the trials in this research, correlations with exertion rating and EMG summation of 0.6625 and 0.5253 , respectively, were found. While the correlations were deemed acceptable, attempts to improve the composite index, based on correlations with exertion rating and EMG summation, were undertaken. Three additional composite index equations $\left(\mathrm{I}_{2}, \mathrm{I}_{3}\right.$, and $\left.\mathrm{I}_{4}\right)$ were developed and evaluated.

$I_{2}=\left(\frac{\cos ^{-1}\left(\frac{F_{z}}{\sqrt{F_{x}^{2}+F_{y}^{2}+F_{z}^{2}}}\right)}{45} * \frac{\sqrt{F_{x}^{2}+F_{y}^{2}+F_{z}^{2}}}{\sqrt{F_{x-M V C}^{2}+F_{y-M V C}^{2}+F_{z-M V C}^{2}}}\right) * 100$

The first of the three equations (Equation 15) built off the same "Normalized Magnitude * Normalized Angle" methodology as Chowdhury et al. (2018), except with only one component. This component was the product of normalized 3-dimensional angle $\mu$ (Equation 10) and the normalized 3dimensional magnitude. 


$$
I_{3}=\left[\frac{\left(\frac{F_{\chi}}{F_{X-M V C}} * \frac{\tan ^{-1}\left(\frac{F_{\chi}}{F_{Z}}\right)}{45}\right)+\left(\frac{F_{y}}{F_{y-M V C}} * \frac{\tan ^{-1}\left(\frac{F y}{F_{Z}}\right)}{45}\right)}{\frac{F_{Z}}{F_{Z-M V C}}}\right]
$$

(Equation 16)

The next equation (Equation 16) was loosely based on the concept of the $\mathrm{T} / \mathrm{C}$ ratio. The dividend in the equation represents the harmful effect of the translational forces, similar to Equation 12 and Equation 15, while the divisor in the equation uses the normalized compressive/distraction force to represent the protective effect of the compressive forces. This was the only equation that was not scaled to 100 , with final values ranging from 0 up to about 4.

$$
I_{4}=\left(\frac{\sqrt{F_{z}^{2}+F_{y}^{2}+F_{z}^{2}}}{\sqrt{F_{z-M V C}^{2}+F_{y-M V C}^{2}+F_{y-M V C}^{2}}} *\left[\frac{\tan ^{-1}\left(\frac{F y}{F_{z}}\right)}{45}+\frac{\tan ^{-1}\left(\frac{F_{x}}{F_{z}}\right)}{45}\right]\right) * 100
$$

Finally, the third equation (Equation 17) is the product of the normalized 3-dimensional magnitude and the sum effect of the frontal (similar to Figure 25, $\beta$ ) and sagittal (similar to Figure 25, $\gamma$ ) planar angles. This equation was based on the same "Normalized Magnitude * Normalized Angle" approach but considered that the magnitude of the individual components is not as influential/important as the magnitude of the 3-component vector.

Additionally, in an effort to further improve the potential composite indices, a physiology-derived stability modifier was developed based on research by Lippitt et al. (1993) that explored shoulder stability using 10 fresh-frozen human cadaveric Glenohumeral joints. In the research, the glenoid was attached securely to a force transducer. The humeral head was placed in the glenoid and a compressive force of $50 \mathrm{~N}$ and, subsequently, $100 \mathrm{~N}$ were applied. While the compressive force was applied, translational forces were applied until dislocation over the labrum occurred from each of 8 directions $\left(0-360^{\circ}\right.$ at $45^{\circ}$ intervals, 
clockwise with $0^{\circ}$ superior). The results of their research provided the percentage the translational load was of the compressive load in each of the 8 directions when the dislocation occurred. A Piecewise Cubic Hermite Interpolating Polynomial function in MATLAB was used to interpolate the values for each $1^{\circ}$ interval. The values were then normalized by dividing by the highest value $\left(64 \%\right.$ at $\left.180^{\circ}\right)$, resulting in values between 0 and 1 representing the amount of stability at each point compared to the point of maximum stability (Figure 25). The stability modifier values for each $1^{\circ}$ increment are available in Appendix $\mathrm{H}$.

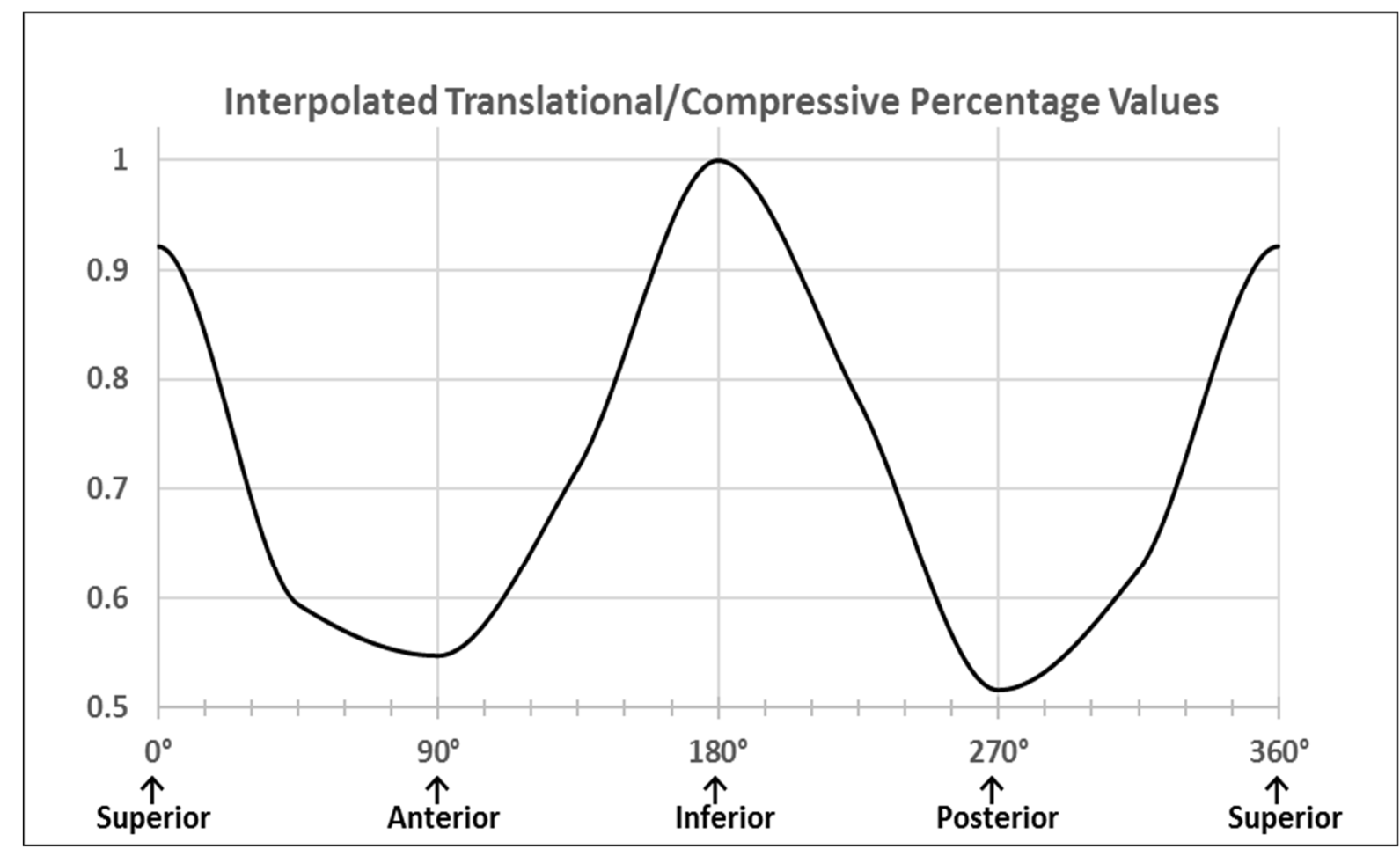

Figure 25. Stability modifier values determined from Lippitt et al. translational/compressive load stability results interpolated with a Piecewise Cubic Hermite Interpolating Polynomial function.

For each experimental trial, the stability modifier value was determined based on the direction of the Glenohumeral reaction force vector in the sagittal plane (i.e. direction of the resultant of anteroposterior and inferosuperior forces) rounded to the nearest degree, when performing the simulation. Each of the four potential composite index equations above was adapted by multiplying each result and dividing each result by the stability modifier value, resulting in a total of 12 potential composite index equations. Correlation analysis between the results of each of the 12 potential composite index equations and exertion rating (Table 10) and EMG summation (Table 11) was performed. 
Table 10. Results of correlation analysis between the perceived exertion values and each of the 12 potential composite index equations.

\begin{tabular}{c|cc|cc|cc}
\hline \multirow{2}{*}{$\begin{array}{c}\text { Composite } \\
\text { Index }\end{array}$} & \multicolumn{2}{c}{ Unmodified } & \multicolumn{2}{c}{ I * StabMod } & \multicolumn{2}{c}{ I / StabMod } \\
\cline { 2 - 7 } & Spearman rho & $p$-Value & Spearman rho & $p$-Value & Spearman rho & $p$-Value \\
\hline $\mathrm{I}_{1}$ & 0.6625 & 0.0000 & 0.6598 & 0.0000 & 0.6628 & 0.0000 \\
$\mathrm{I}_{2}$ & 0.6664 & 0.0000 & 0.6632 & 0.0000 & 0.6661 & 0.0000 \\
$\mathrm{I}_{3}$ & 0.6573 & 0.0000 & 0.6453 & 0.0000 & 0.6643 & 0.0000 \\
$\mathrm{I}_{4}$ & 0.6662 & 0.0000 & 0.6648 & 0.0000 & 0.6650 & 0.0000 \\
\hline
\end{tabular}

Table 11. Results of correlation analysis between the EMG summation values and each of the 12 potential composite index equations.

\begin{tabular}{c|cc|cc|cc}
\hline \multirow{2}{*}{$\begin{array}{c}\text { Composite } \\
\text { Index }\end{array}$} & \multicolumn{2}{c}{ Unmodified } & \multicolumn{2}{c}{ I * StabMod } & \multicolumn{2}{c}{ I / StabMod } \\
\cline { 2 - 7 } & Pearson $r$ & $p$-Value & Pearson $r$ & $p$-Value & Pearson $r$ & $p$-Value \\
\hline $\mathrm{I}_{1}$ & 0.5253 & 0.0000 & 0.5231 & 0.0000 & 0.5224 & 0.0000 \\
$\mathrm{I}_{2}$ & 0.5453 & 0.0000 & 0.5432 & 0.0000 & 0.5420 & 0.0000 \\
$\mathrm{I}_{3}$ & 0.4741 & 0.0000 & 0.4662 & 0.0000 & 0.4757 & 0.0000 \\
$\mathrm{I}_{4}$ & 0.4294 & 0.0000 & 0.4276 & 0.0000 & 0.4285 & 0.0000 \\
\hline
\end{tabular}

Since all unmodified composite index equations had similar correlations with the EMG summation and perceived exertion ratings, the decision was made to continue forward with potential composite index $I_{1}$, the index developed by Chowdhury et al. (2018), due to the validation of the equation done within their research. Multiplying the result of this composite index by the Stability Modifier resulted in decreased correlation with the perceived exertion rating and EMG summation. Dividing the result of this composite index by the Stability Modifier resulted in a slight increase in the correlation with the perceived exertion rating, but the correlation with EMG summation decreased. Therefore, the unmodified $\mathrm{I}_{1}$ composite index was chosen.

\subsection{Predictive Equation Development}

For each experimental trial, the distance from the sternal notch to the location of the load was calculated along the $\mathrm{X}, \mathrm{Y}$, and $\mathrm{Z}$ axes (Figure 26) by subtracting the coordinates of the CLAV marker on the sternal notch from palm-center of the hand, defined as the average of the four markers on the wrist/hand 
(RWRA, RWRB, RTHB, and RFIN markers). Additionally, marker coordinate values were output from Vicon Nexus in millimeter units, so each value was divided by 25.4 to convert from millimeters to inches. The following sets of predictors were derived from these values:

1. 3-Direction Distances: This set of predictors were the raw distances along the $\mathrm{X}, \mathrm{Y}$, and $\mathrm{Z}$ axes in units of inches.

$X$-axis (+Forward/-Backward): Distance forward/backward from the sternal notch to palm-center of the hand. Positive values are distances forward. Negative values are distances backward.

Y-axis (+Right / -Left): Distance right/left between from the sternal notch to palm-center of the hand. Positive values are distance to the right. Negative values are distances to the left.

Z-axis (+Up/-Down): Distance up/down from the sternal notch to palm-center of the hand. Positive values are distances above the sternal notch. Negative values are distances below the sternal notch.

2. 6-Direction Distances: This set of predictors were distances in 6 directions (Forward, Backward, Right, Left, Up, and Down) along the $\mathrm{X}, \mathrm{Y}$, and $\mathrm{Z}$ axes in units of inches. For example, if the 3Direction Distance Y-axis value was -8.75, the 6-Direction Distances for Right and Left would be 0 and 8.75, respectively. This allowed different regression coefficients for forward and backward, right and left, and up and down.

3. Normalized 3-Direction Distances - Norm. Direction: This set of predictors were the 3-Direction Distances normalized by the height of the participant, by dividing each predictor by the participant's height in inches. 


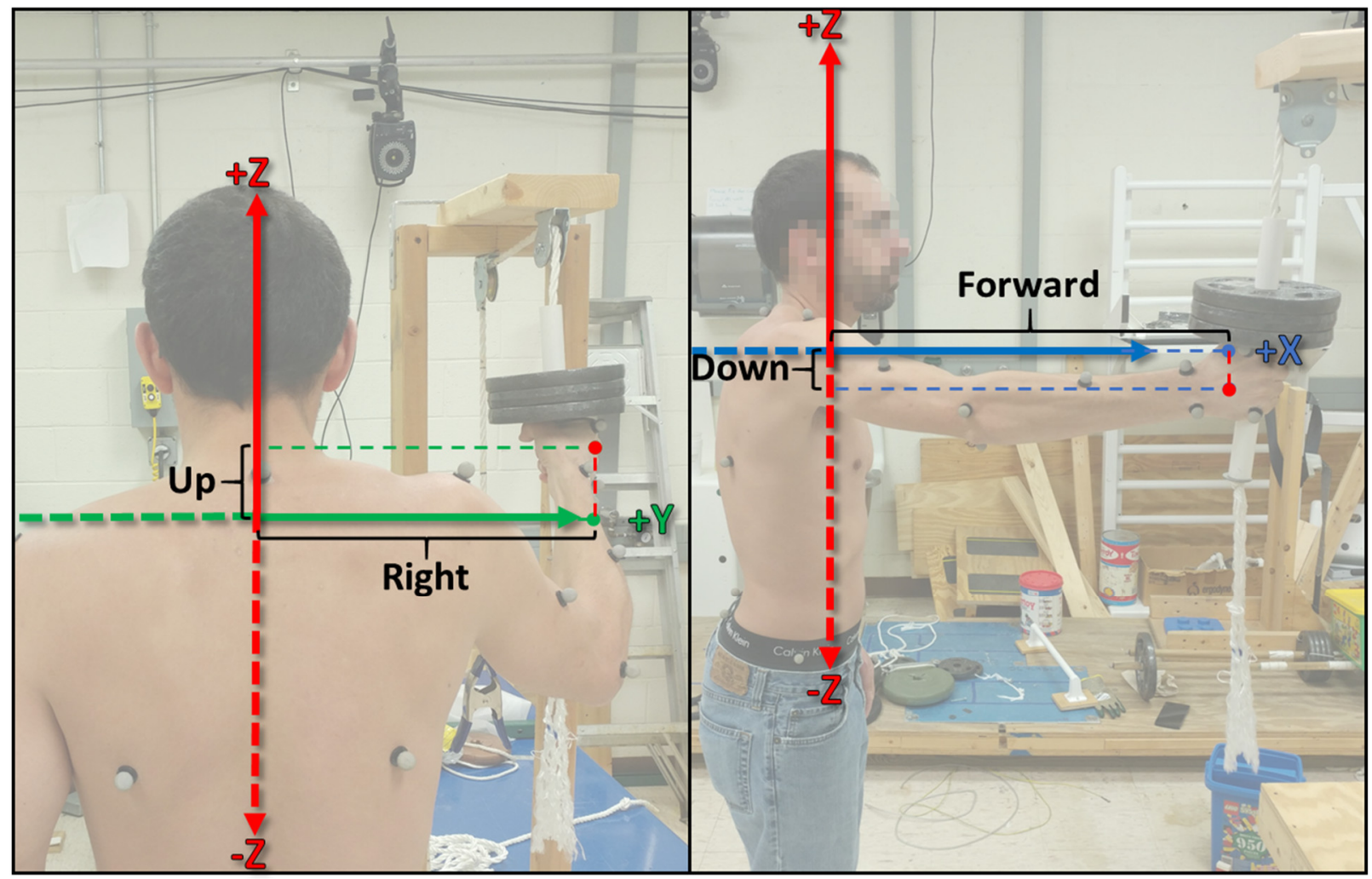

Figure 26. Representation of how the distances between the sternal notch and load are measured along each axis.

4. Normalized 6-Direction Distances-Norm. Direction: This set of predictors were the 6-Direction Distances normalized by the height of the participant, by dividing each predictor by the participant's height in inches.

5. 3-Direction Square Roots - SQRT(Direction): This set of predictors were the square roots of the absolute values of the 3-Direction Distances with the direction sign maintained. For instance, if the 3-Direction Distance Y-axis value was -9.0, the 3-Direction Square Root Y-axis value would then be -3.0 .

6. 6-Direction Square Roots -SQRT(Direction): This set of predictors were the square roots of the 6-Direction Distances. 
7. 3-Direction Absolute Values - ABS(Direction): This set of predictors were the absolute values of the 3-Direction Distances

8. 3-Direction Squares-Direction ${ }^{2}$ : This set of predictors were the 3-Direction Distances Squared.

9. 6-Direction Squares-Direction ${ }^{2}$ : This set of predictors were the 6-Direction Distances Squared.

10. 3-Direction Cubes-Direction ${ }^{3}$ : This set of predictors were the 3-Direction Distances Cubed.

11. 6-Direction Cubes-Direction ${ }^{3}$ : This set of predictors were the 6-Direction Distances Cubed.

Best Subsets Regression analysis in Minitab 17.0 (Minitab Inc., State College, PA, USA) was used to determine which of these predictors should be given focus. However, due to the assumption of no multicollinearity between factors and the high correlation between most sets of these predictors, each set of predictors had to be evaluated separately. Since the purpose of this regression analysis was to find an equation that can accurately predict the composite index values, the values for the predicted R-square of each predictor set were compared. For each set of predictors, the best single predictor and the number of factors in the best subset of predictors with the highest predicted R-square values are shown in Table 12. See Appendix I for full results of Best Subset Regression analysis.

Based on the results of the Best Subsets Regression analysis of the 11 sets of predictors, the strongest single predictor (Predicted R-Square $=34.6 \%$ ) was the square root of the 3-Direction Distance $+U p /-D o w n$. The predictor set with the best subset of predictors (Predicted R-Square $=65.7 \%$ ) were the 6Direction Distance values. The best subset of this predictor set included 5 predictors, with the exclusion being distance backward. This was expected, as a distance backward, signifying that the hand was behind the clavicle, was 
Table 12. Summary results from the Best Sets analysis, including the best single predictor and the number of predictors in the Best Set of each subset of the 11 sets of predictors.

\begin{tabular}{clc|cc}
\hline \multirow{2}{*}{ Predictor Set } & \multicolumn{2}{c}{ Single Predictor } & \multicolumn{2}{c}{ Best Subset } \\
\cline { 2 - 4 } & \multicolumn{1}{c|}{ Best Predictor } & Pred. R-Square & \# of Predictors & Pred. R-Square \\
\hline $\mathbf{1}$ & +Up / -Down & $34.0 \%$ & 2 & $61.0 \%$ \\
$\mathbf{2}$ & Down & $32.4 \%$ & 5 & $65.7 \%$ \\
$\mathbf{3}$ & Norm +Up / -Down & $33.9 \%$ & 2 & $60.7 \%$ \\
$\mathbf{4}$ & Norm Down & $32.6 \%$ & 5 & $65.2 \%$ \\
$\mathbf{5}$ & SQRT(+Up / -Down) & $\mathbf{3 4 . 6 \%}$ & 3 & $54.6 \%$ \\
$\mathbf{6}$ & SQRT(Down) & $33.7 \%$ & 6 & $60.7 \%$ \\
$\mathbf{7}$ & ABS(+Fwd / -Bwd) & $32.5 \%$ & 2 & $36.4 \%$ \\
$\mathbf{8}$ & (+Fwd / -Bwd) & $33.7 \%$ & 2 & $35.9 \%$ \\
$\mathbf{9}$ & Fwd & 6 & $61.1 \%$ \\
$\mathbf{1 0}$ & (+Fwd / -Bwd) $^{3}$ & $31.6 \%$ & 2 & $49.4 \%$ \\
$\mathbf{1 1}$ & Fwd $^{3}$ & $31.6 \%$ & 6 & $52.5 \%$ \\
\hline
\end{tabular}

an uncommon case, representing only 21 of the 354 total trials. These cases occurred when the palm-center was intended to be along the frontal plane, i.e. directly to the right or left of the participant and the slight variations in lifting resulted in the palm-center being slightly behind the clavicle along the $\mathrm{X}$-axis.

Based on these results, a Stepwise Regression ( $\alpha$ for entry/removal $=0.15$ ) analysis was performed with the following terms: Forward, Right, Left, Up, Down, SQRT(Up), SQRT(Down), Square terms of each ( 7 terms), and all 2-way interactions (21 terms). Sixteen terms remained in the regression equation, and a Predicted R-Square value of $75.71 \%$ was found. See Appendix J1 for the full regression analysis result. Since SQRT(Up) and SQRT(Down) are better predictors than Up and Down, respectively, the Up and Down terms were removed from the analysis, though none of the interaction terms involving Up and Down were removed. The analysis was performed again without these terms and a regression equation with a Predicted R-Square of $75.68 \%$ and only 8 terms was found (Appendix J2).

Further attempts at optimizing and improving the predictive equations, i.e. removing the square terms (Predicted R-Square $=74.18 \%, 9$ terms) and reducing the $\alpha$ for entry/removal for the stepwise 
regression to 0.10 (Predicted R-Square $=74.22 \%, 8$ terms), showed no further improvements (Appendix J3 and J4). Since these four regression equations performed similarly, additional correlation analysis was performed and Root Mean Square Error (RMSE) values were calculated (Table 13).

Regression Equation 1 had the highest Predicted R-Square, the strongest correlation with the composite index values, and the lowest RMSE, while the strongest correlation with the perceived exertion values was found with Regression Equation 2. While Regression Equation 1 performs the best, the usability of the equation was also considered. Since Regression Equation 2 performs nearly as well as

Table 13. Correlations between the predicted strain values data using each regression equations and the composite index values, calculated from the participant data, and perceived exertion rating; RMSE calculated between composite index values and predicted strain values for each regression equation.

\begin{tabular}{ccc|cc|c}
\hline \multirow{2}{*}{$\begin{array}{c}\text { Regression } \\
\text { Equation }\end{array}$} & $\begin{array}{c}\text { Predicted } \\
\text { R-Square }\end{array}$ & \multirow{2}{*}{ Terms } & \multicolumn{2}{|c|}{ Correlations } & RMSE \\
\cline { 4 - 6 } & & Composite Index & Perceived Exertion & Composite Index \\
\hline $\mathbf{1}$ & $75.71 \%$ & 16 & 0.8838 & 0.6235 & 7.8291 \\
$\mathbf{2}$ & $75.68 \%$ & 8 & 0.8783 & 0.6244 & 8.0029 \\
$\mathbf{3}$ & $74.18 \%$ & 9 & 0.8708 & 0.6138 & 8.2276 \\
$\mathbf{4}$ & $74.22 \%$ & 8 & 0.8700 & 0.6109 & 8.2531 \\
\hline
\end{tabular}

Regression Equation 1 with only half as many terms, Regression Equation 2 was accepted as the final Predictive Equation:

$C I=12.41-(2.597 \sqrt{D})+\left(0.08620 F^{2}\right)+\left(0.02886 R^{2}\right)-\left(0.3638 U^{2}\right)+(0.2004 F L)-(0.2110 R \sqrt{U})+$ $(0.1262 R \sqrt{D})+\left(1.849 U^{3 / 2}\right)$

Where,

$$
\begin{aligned}
& \mathrm{F}=\text { Distance forward (Inches) } \\
& \mathrm{R}=\text { Distance right (Inches) } \\
& \mathrm{L}=\text { Distance left (Inches) } \\
& \mathrm{U}=\text { Distance up (Inches) } \\
& \mathrm{D}=\text { Distance down (Inches) }
\end{aligned}
$$

In an effort to condense the equation, the coefficients were each rounded slightly, resulting in no change in the correlation with composite index $(r=0.8783)$ and a small decrease in correlation with the perceived exertion ( $\rho=0.6237$ vs. $\rho=0.6244$ ) and only a small increase in the RMSE between the 
calculated and predicted composite index values (8.0029 vs. 8.0031). The final predictive equation is as follows:

$$
C I=12.4-(2.6 \sqrt{D})+\left(0.086 F^{2}\right)+\left(0.029 R^{2}\right)-\left(0.364 U^{2}\right)+(0.2 F L)-(0.21 R \sqrt{U})+(0.126 R \sqrt{D})+\left(1.85 U^{3 / 2}\right)
$$

Where,

$$
\begin{aligned}
& \mathrm{F}=\text { Distance forward (Inches) } \\
& \mathrm{R}=\text { Distance right (Inches) } \\
& \mathrm{L}=\text { Distance left (Inches) } \\
& \mathrm{U}=\text { Distance up (Inches) } \\
& \mathrm{D}=\text { Distance down (Inches) }
\end{aligned}
$$

When using this equation, for the Left/Right and Up/Down pairs, the opposing of each pair should be set to 0 , i.e. If the distance is 5.5 inches to the right, $\mathrm{R}=5.5$ and $\mathrm{L}=0$.

\subsection{Heatmaps}

Predicted strain values were classified into the following risk ranges: Low: 0-20, Medium: 20-60, and High: 60-100. Since magnitude of the load is not considered by these predictive equations, despite being a major factor in the overall risk of shoulder strain, a high predicted strain based on the location may not estimate actual risk, especially as weight of the load increases above or decreases below the 15 pound weight used to develop the predictive equations. To provide additional visual guidance, MATLAB R2017b (Mathworks Inc., Natick, MA, USA) was used to create heatmaps of the predicted strain estimated by the predictive equations. Heatmaps were created for 9 different heights: 20, 15, 10, and 5 inches below the sternal notch, vertically aligned with the sternal notch, and 5, 10, 15, and 20 inches above the sternal notch (Figures 27-35). For each height, the distances forward/backward and left/right were divided up into 0.5inch intervals, and the intervals within the reach envelope were approximated, which were based on the peak distance values from the collected data. For each interval, the predicted strain was calculated from the predictive equation. Full-page heatmaps are available in Appendix K. 


\subsection{Discussion}

During the development and analysis of the composite index equations, the resulting correlation values were lower than ideal; there were several potential reasons for this. The composite index values certainly had variation due to scaling of the models based on height and weight affecting the reference values used in the composite index equations, and this variability could not be accounted for or controlled during correlation analysis. Additionally, the perceived exertion was a subjective metric and therefore is likely to have some variability, especially between participants, but also between trials of the same participants. However, options for validation are otherwise limited. Multiple repetitions of the experimental tasks would provide some control over the variance, but without more control over the lifting location, it

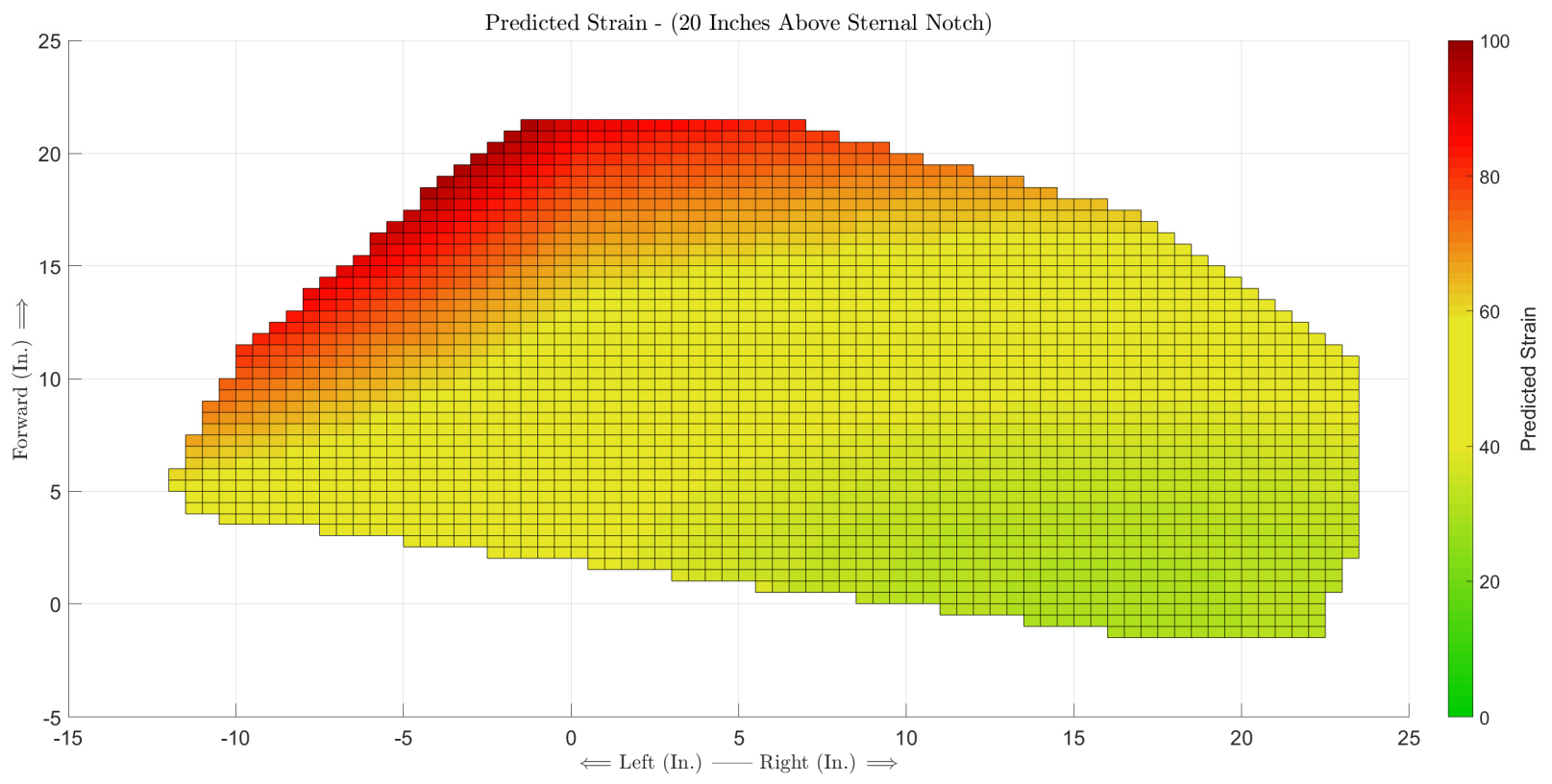

Figure 27. Predicted Strain with the load 20 inches above the sternal notch. 


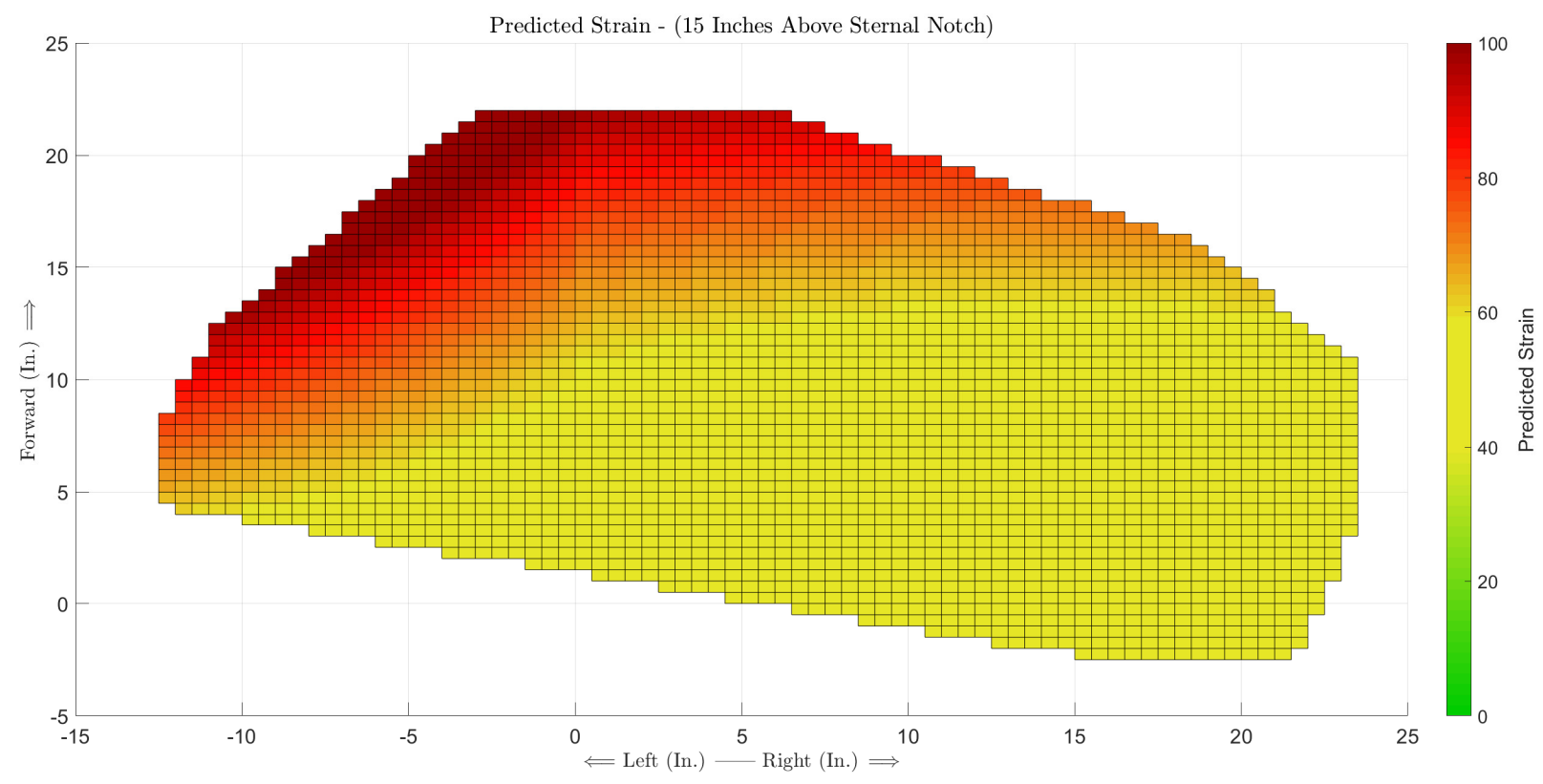

Figure 28. Predicted Strain with the load 15 inches above the sternal notch.

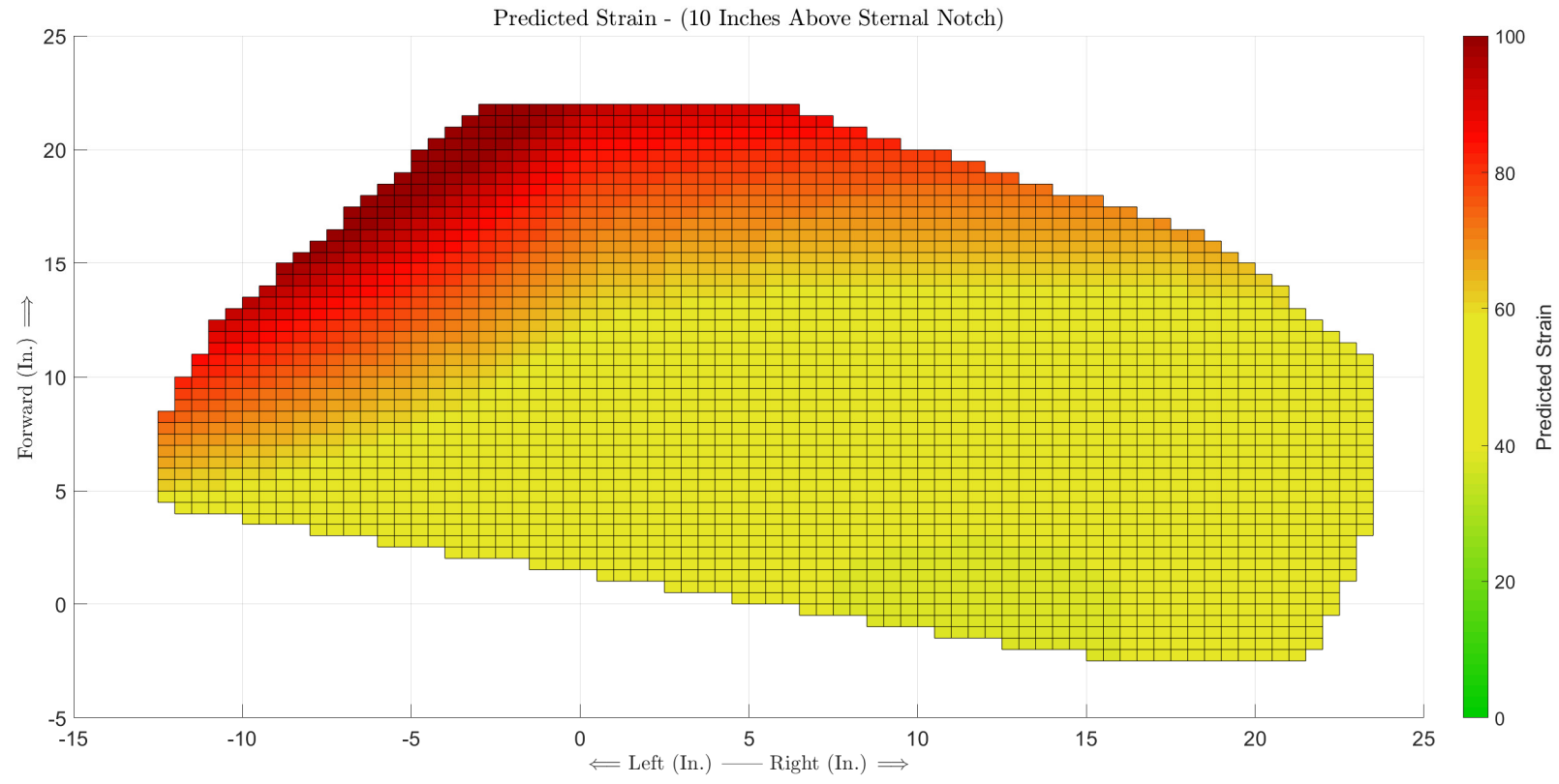

Figure 29. Predicted Strain with the load 10 inches above the sternal notch. 


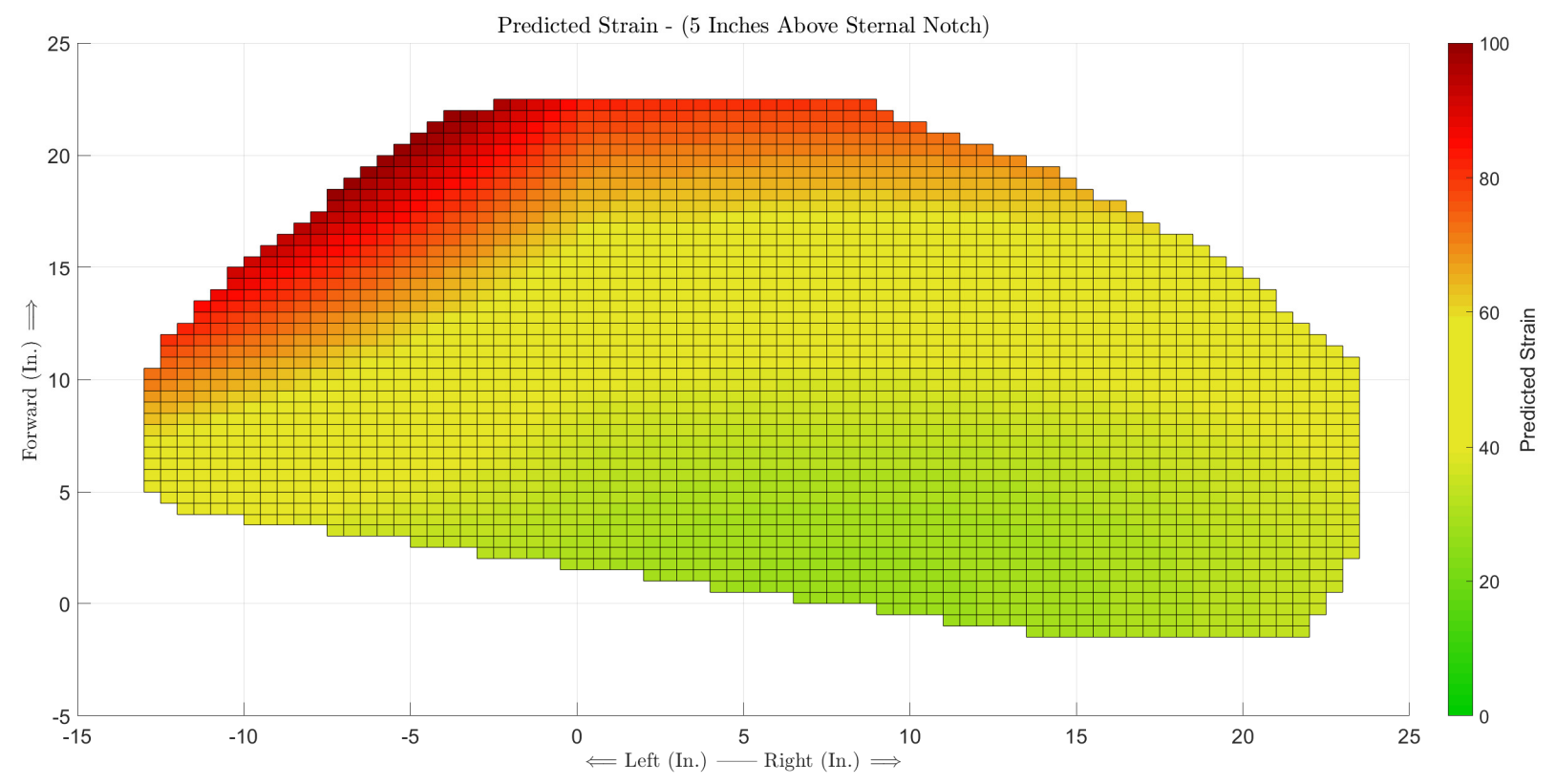

Figure 30. Predicted Strain with the load 5 inches above the sternal notch.

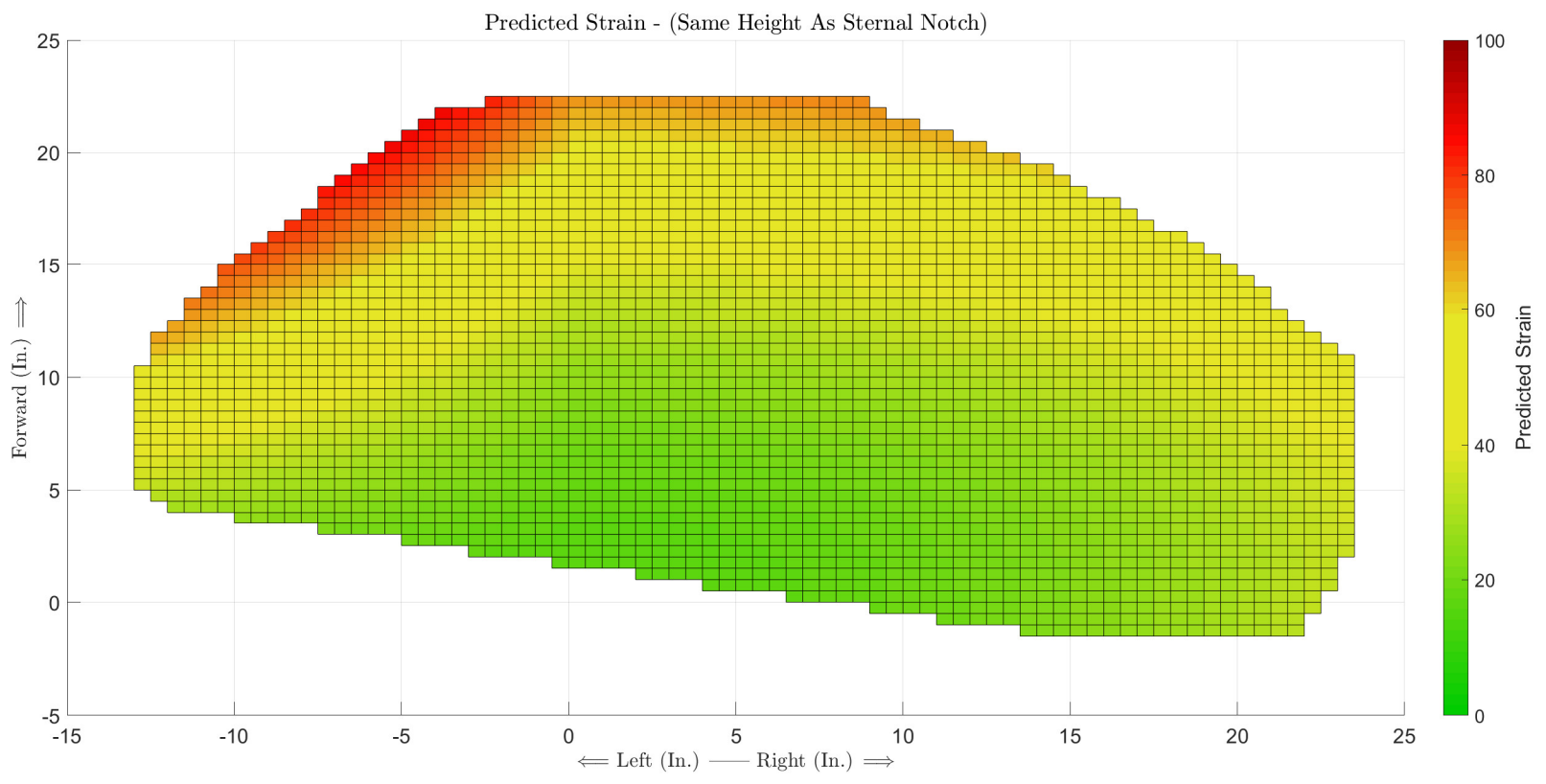

Figure 31. Predicted Strain with the load horizontally aligned with the sternal notch. 


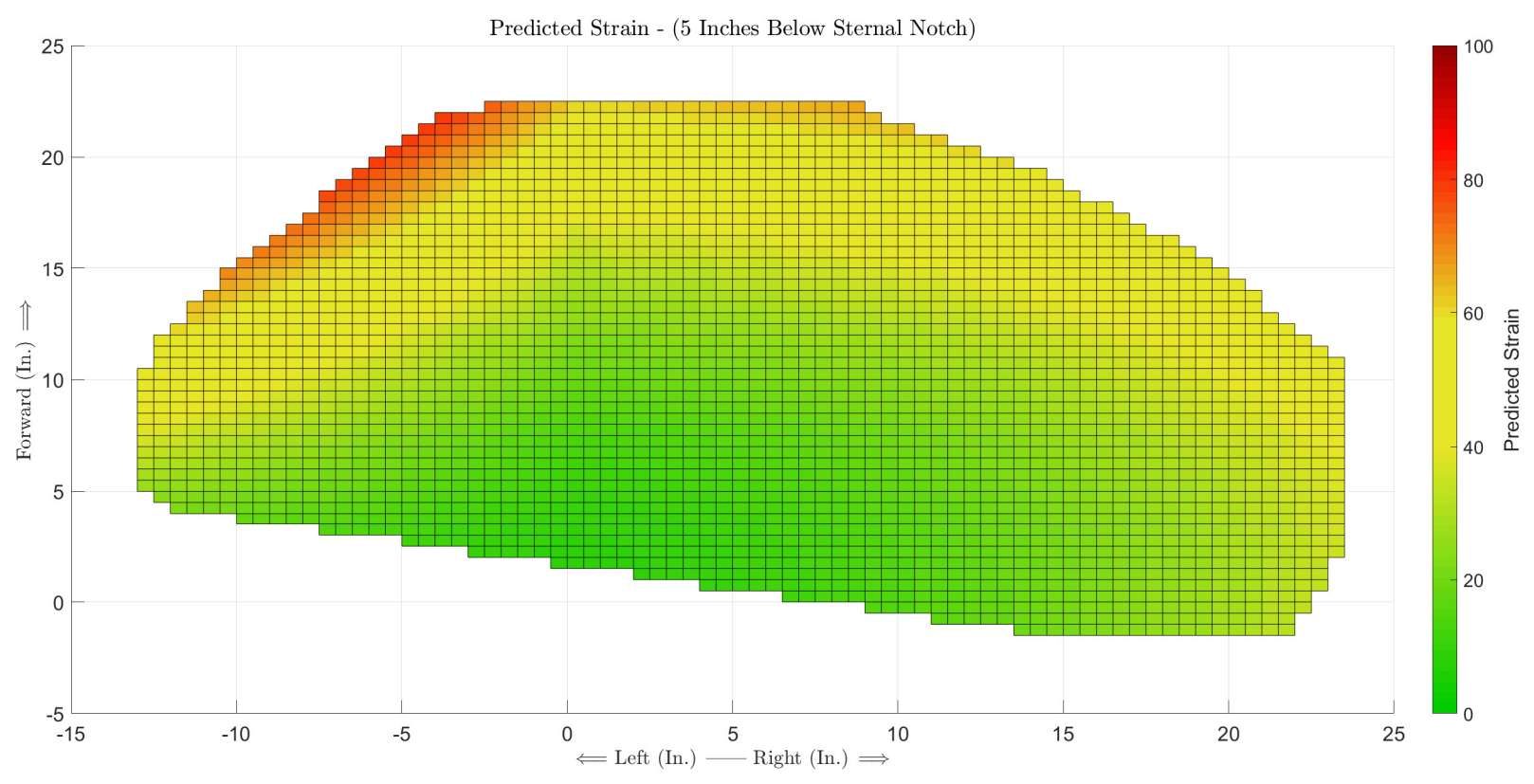

Figure 32. Predicted Strain with the load 5 inches below the sternal notch.

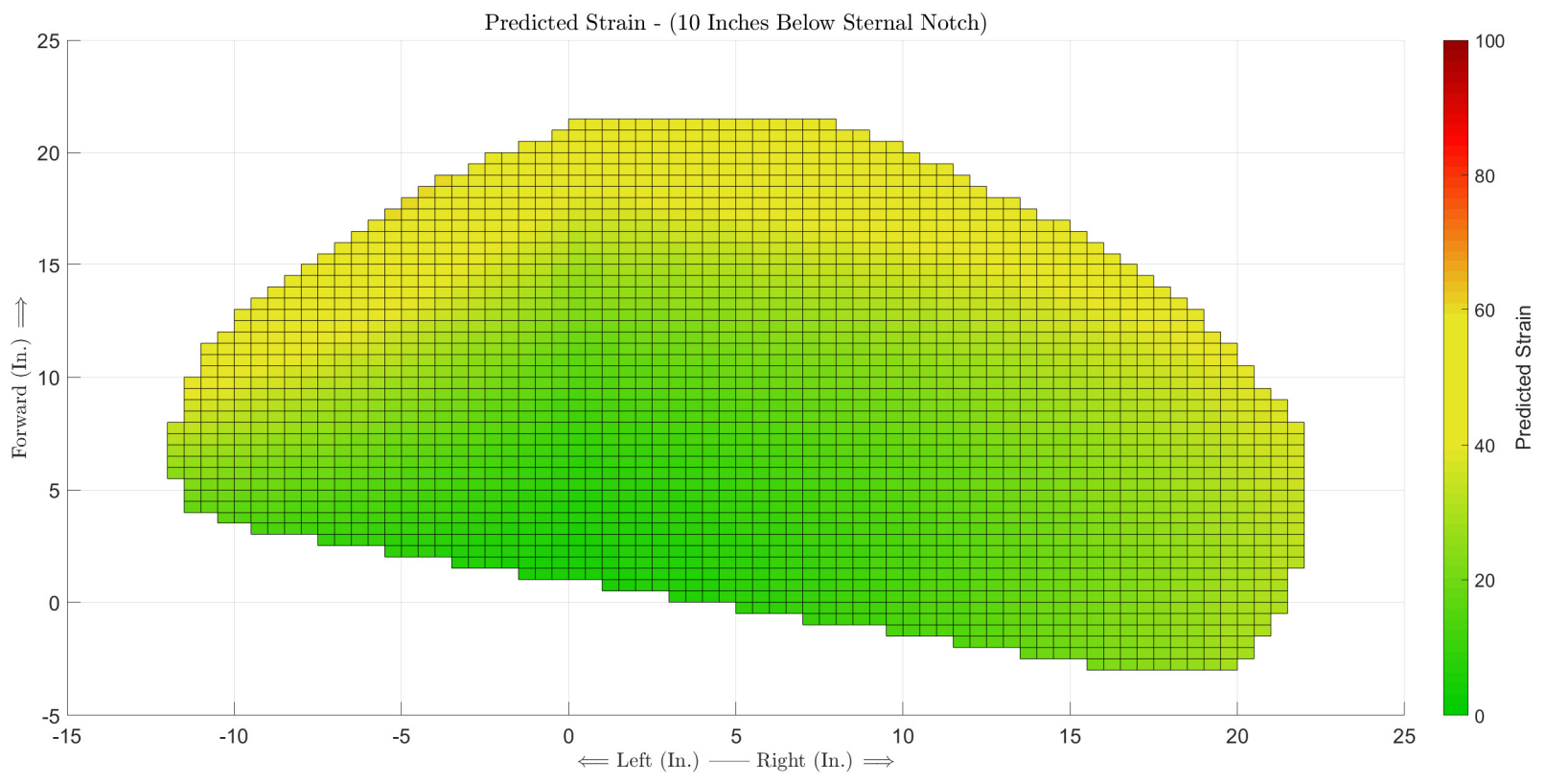

Figure 33. Predicted Strain with the load 10 inches below the sternal notch. 


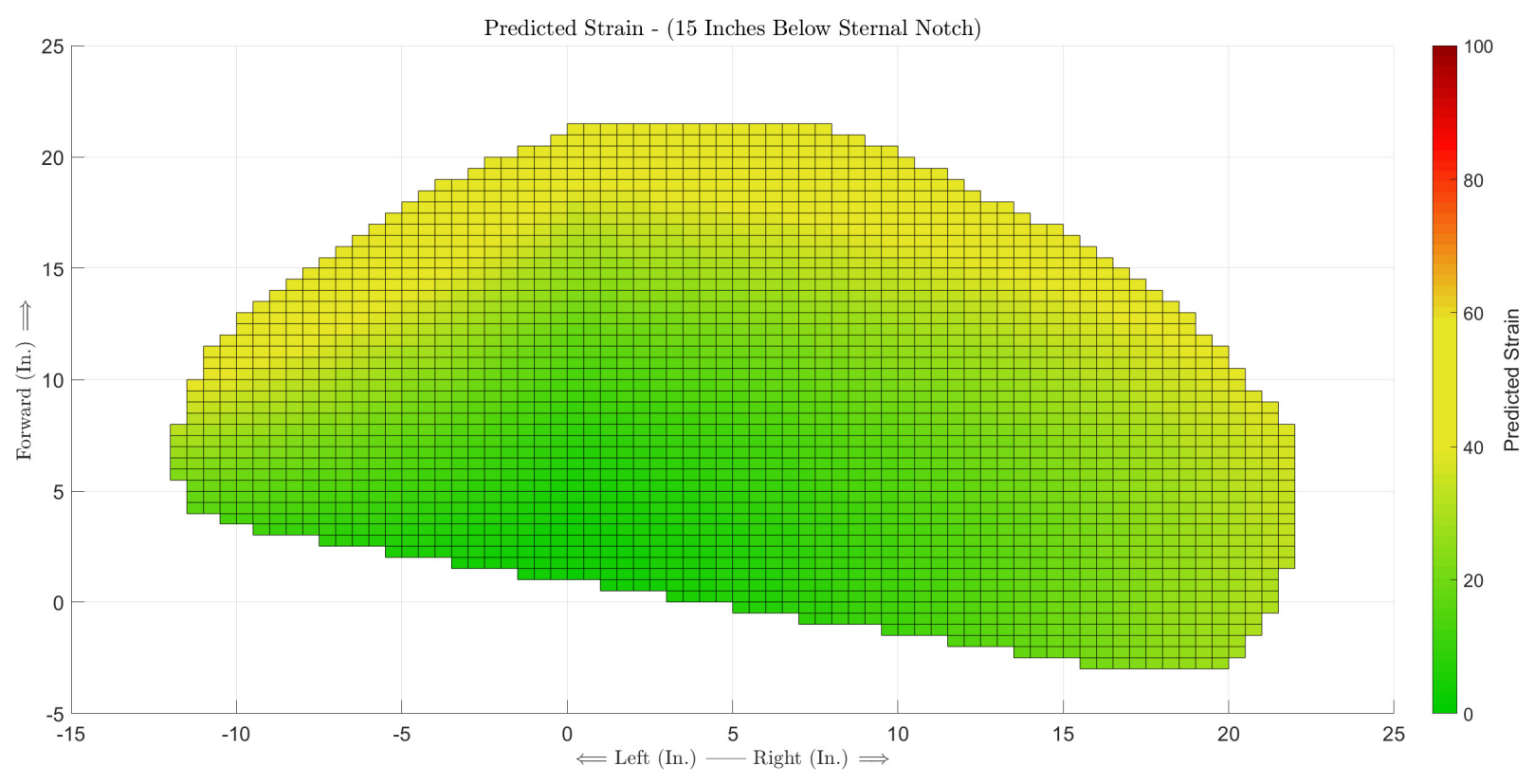

Figure 34. Predicted Strain with the load 15 inches below the sternal notch.

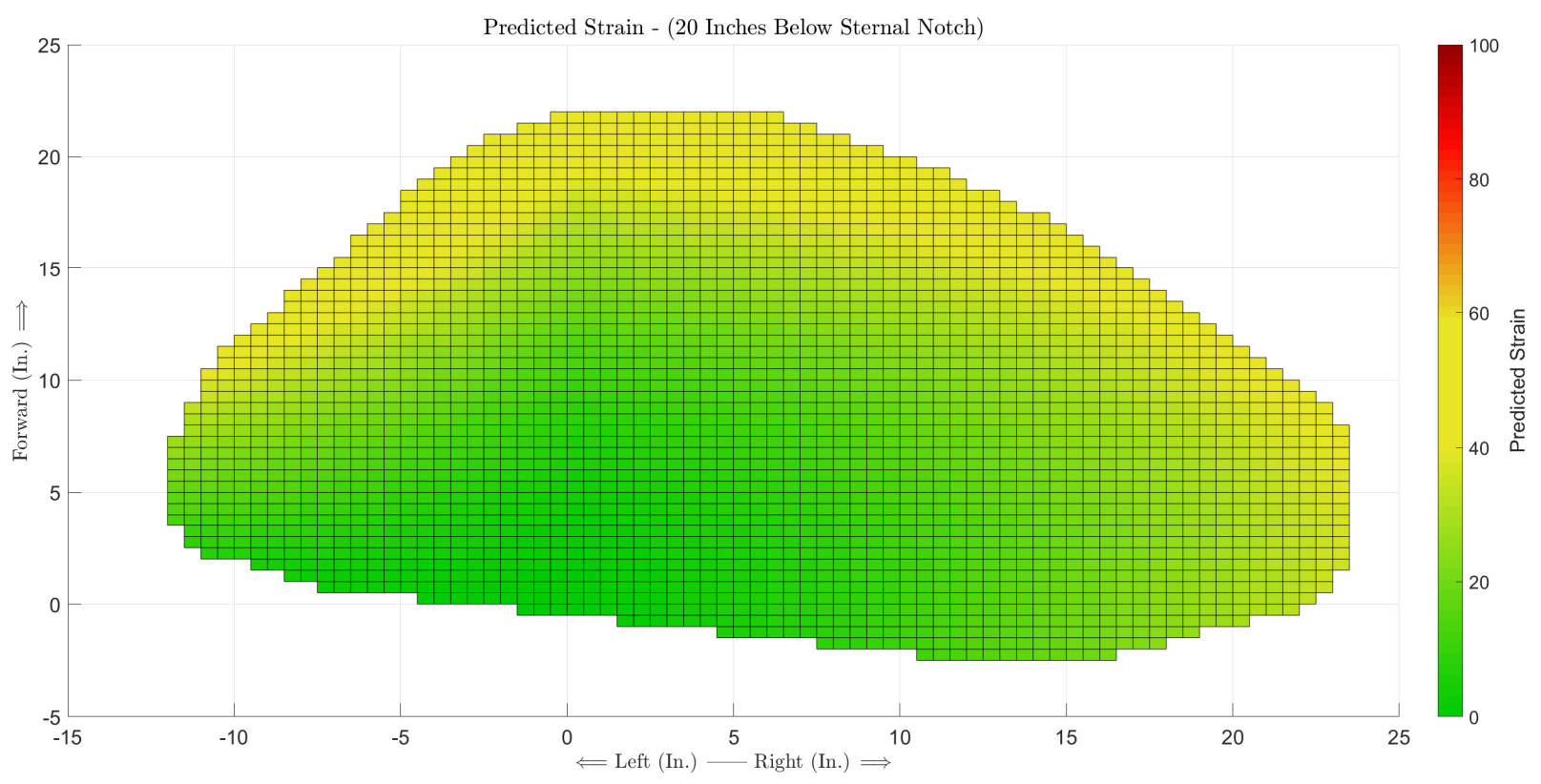

Figure 35. Predicted Strain with the load 20 inches below the sternal notch. 
would not be wise to take the mean of the trials that are supposed to be from the same location. It would be more prudent, instead, to decrease the lift location intervals, e.g. 4" horizontal intervals and/or 5" vertical intervals and perform a separate analysis for each participant.

Despite one of the composite index equations developed for this research having a slightly higher correlation with the EMG summation and perceived exertion, the composite index developed by Chowdhury et al. (2018) was used due to its more extensive validation. There was also a slight improvement when the stability modifier was included, however the improvement was so modest that the increased complexity of the calculation did not make it worthwhile. Unexpectedly, however, the correlations were similar across the composite indices, despite being drastically different calculations. In the case of the Spearman rank correlation between the perceived exertion ratings and composite index values, this was not surprising, due to the ordinal nature of the perceived exertion rating. Since the EMG summation was a continuous variable and Pearson correlation was used, the correlation values had greater variance between the potential composite indices, though the differences were still relatively small. Despite these differences, the results of this analysis support the use of the principles of the concavity-compression mechanism for prediction of shoulder strain and provides physiological support for the predictive equation.

Development of the predictive equations presented a challenge, due to the non-typical use of regression methods. Since our regression predictors had to be derived from three values (distance along X, $\mathrm{Y}$, and $\mathrm{Z}$ axes from sternal notch to load), issues of collinearity required multiple separate regression analyses and a novel approach to the development of the predictive equation, first using best sets regression. Despite these difficulties, the resulting predictive equations had good predictability (Predicted R-Sq $\approx 75 \%$ ) and strong correlation with the accepted composite index $(\rho \approx 0.88)$. The calculated RMSE values (RMSE $\approx 8$ ) between the strain calculated with the predictive equations and the composite index results were acceptable, considering the application. A discrepancy of 8 points in the predicted strain, in a range of 0 100 , would represent a small difference in risk that is unlikely to have real-world implications. 
The heatmaps developed from the predictive equation support our current understanding of risk factors of shoulder strain. First, the strain increases as the horizontal distances (forward, left/right) increase. This is explained by the increased moment arm as the horizontal distance between the load and the shoulder increases. Next, we notice the strain increases as the height of the load increases, with the increase accelerating once shoulder level is reached. Lifting above shoulder level is a well-established risk factor for shoulder injury (Bodin et al., 2012; Faber et al, 2009; Ferguson et al, 2013; Harkness et al, 2003; Hughes et al, 1997; Leclerc et al, 2004) so this trend was expected. Additionally, an increase in the strain when lifting across the body was found, but only when the load was not near the body. This suggests an interaction between lifting across the midline and a larger moment arm. While this has not been explored in the literature as a risk factor of shoulder injury, these heatmaps suggest that it should possibly be explored as such. 


\section{SPECIFIC AIM \#3: VALIDATION OF PREDICTIVE EQUATION AS TASK \\ ANALYSIS TOOL}

\subsection{Objectives and Hypothesis}

When performing a manual material handling task that involves moving an object from one location to the other within the worker's functional working range, the origin and destination of the object are hypothesized to be the most strenuous points of the motion. The moment arm, i.e. horizontal distance between the joint of interest and the load, has a major influence on the reaction forces at the joint. As the moment about a joint increases, more muscle activity is required to counteract the moment, resulting in increased forces at the joint. Generally, when moving a load, the moment arm should be the highest at either the origin or the destination; a) when the destination is farther away than the origin, the load is not extended farther than the destination, b) when a load is moved across the body the moment will reduce as the object is passed in front of the body and increase again to the destination, and c) when moving a load vertically, the moment will increase as the load height increases, and the load won't be raised higher than needed. Based on this, when moving an object through the working range, the object will be moved along a path that brings the object into more physiologically favorable positions unless an obstacle exists. The NIOSH lifting equation uses a similar origin/destination analysis principle to analyze tasks from the perspective of low-back safety, and cited moment arm concerns as a justification for their methods (NIOSH, 1981).

Objective 1: Validate use of the predictive equation at the origin and destination of a task as an appropriate task analysis tool by ensuring that the strain on the shoulder is highest at either the origin or destination of the lift by plotting composite index values calculated throughout a lifting task.

Null Hypothesis: Estimated Composite Index values are highest at the origin or destination of the load during an unobstructed lifting task.

Alt. Hypothesis: Strain varies throughout the unobstructed lifting task with locations other than the origin or destination having the highest estimated Composite Index values. 


\subsection{Approach}

Initially, further validation of the predictive equation was performed using correlation analysis and calculating root mean square error between the composite index values calculated from AnyBody Modeling System output and the predicted strain calculated using the Predictive Equation using data collected during this Aim.

Next, the hypothesis was tested. Using the heatmaps, two locations rated at each of the three risk levels (low, medium, and high) within the reach envelope of the right arm were selected. While being recorded by motion capture, recruited participants performed lifting tasks between these locations. Experimental trials were modeled in AnyBody Modeling System, and results were used to calculate the Composite Index values using the equation developed by Chowdhury et al. (2018). Additionally, motion capture marker coordinates were used to calculate the location of the load, and the Predictive Equation developed in Specific Aim 2 was used to calculate predicted shoulder strain throughout each lifting task. For each lifting task, the predicted strain at the origin and destination will be compared to the max predicted strain during the task. If the max predicted strain throughout the lifting task occurs at either the origin or the destination, then the use of the strain at the origin/destination to analyze the overall shoulder strain during the task is supported.

\subsection{Participants}

A convenience sample of five new participants with mean age, height, and weight of $27.6( \pm 6.0)$ years, $1.77( \pm 0.03)$ meters, and $82.6( \pm 19.4) \mathrm{kg}$, respectively, were recruited for this study. The sample size was limited to 5 participants because no tests of significance or hypothesis testing was performed, and five participants was sufficient to determine if significant variations in lifting style/technique exist. Ultimately, variations in lifting and handling technique/style should account for most of the differences in the results since the data is model-derived. For inclusion in the study, potential participants were required to be male, aged 18-40 years old, and have no musculoskeletal disorders that would affect their ability to perform the 
tasks or that could affect the results. Male participants were used for the same reasons given in Section 2.4. Participants were confirmed to be right-hand dominant.

\subsection{Equipment}

4.4.1 Optical Motion Capture System (MX Series, Vicon Motion Systems, Oxford, UK): See Section 2.5.1.1

4.4.2 Custom Adjustable Tripod Shelves: Two tripods were used as bases for the adjustable shelves (Figure 36a). Two 4" x 4" wooden "shelves" were cut from 3/4" plywood (Figure 36b). Pronged T-nuts were embedded at the midpoint of each shelf to allow them to be attached to tripods (Figure 36c). Tripods were measured and marked in various configurations to allow them to be quickly and accurately adjusted to the desired height based on the participant's sternal notch height (Figure 36d).

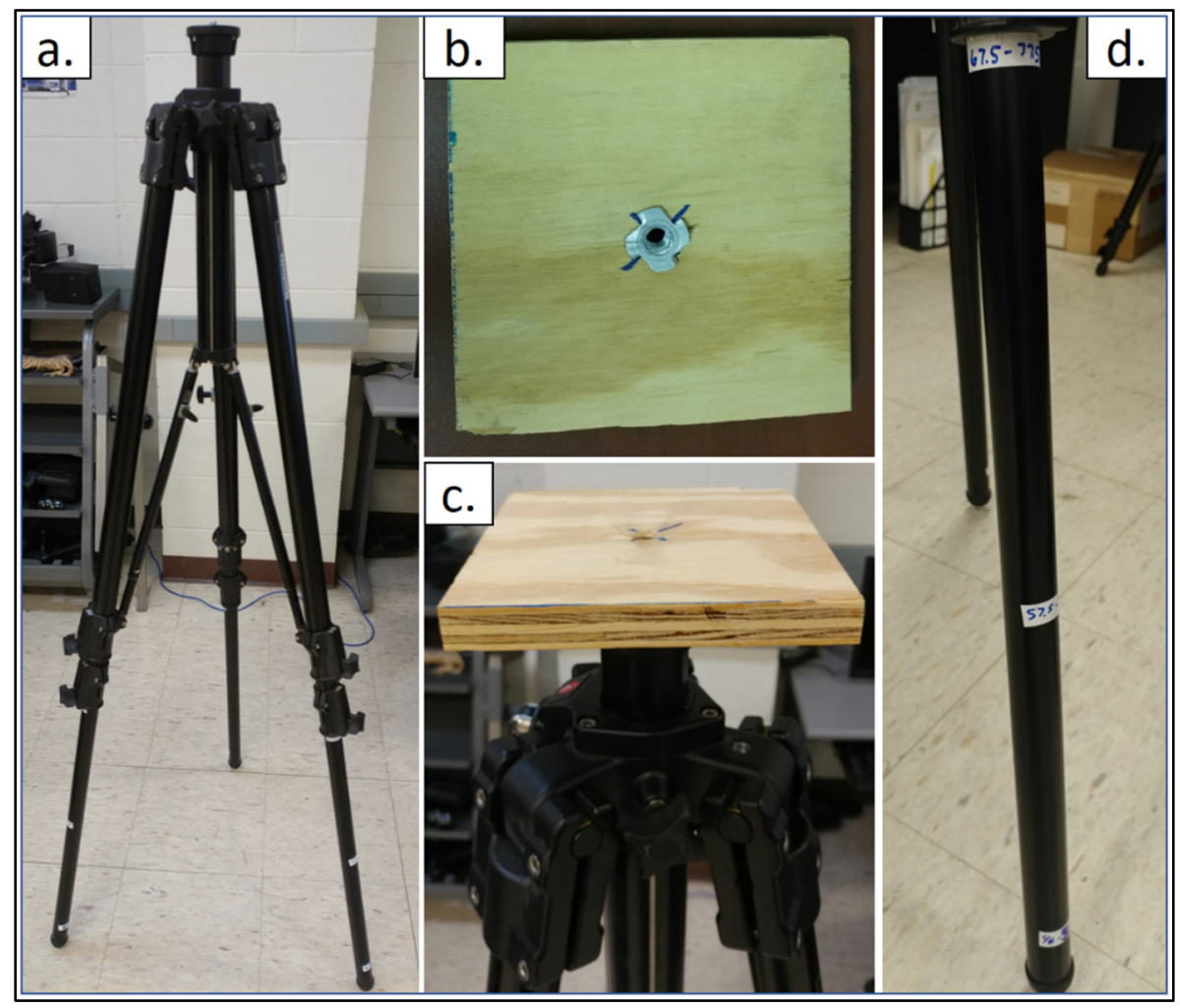

Figure 36. a) One of two matching tripods used as part of the adjustable shelf system. b) One of two 4" by 4" wooden platforms with pronged T-nut embedded. c) Wooden platform attached to tripod. d) Height range markings to allow easy height adjustability of tripods. 
A small "X" was made on the floor at a central location. The participant's sternal notch (CLAV marker) was aligned to this mark during the experimental trials. Additional marks were placed on the floor at the forward and left/right coordinate of each of the 6 load locations.

4.4.3 Weight: Two 5-pound plate weights were bolted to a D-handle to act as the 10-pound load (Figure 37) during the experimental tasks. A retro-reflective marker was affixed to the handle to track the motion of the weight.

\subsection{Experimental Design}

The heatmaps developed in Specific Aim 2 were used to determine two locations each of low strain (Low 1, Low 2), medium strain (Medium 1, Medium 2), and high strain (High 1, High 2). The relative distances between these locations and the sternal notch, and the predicted strain at each location are

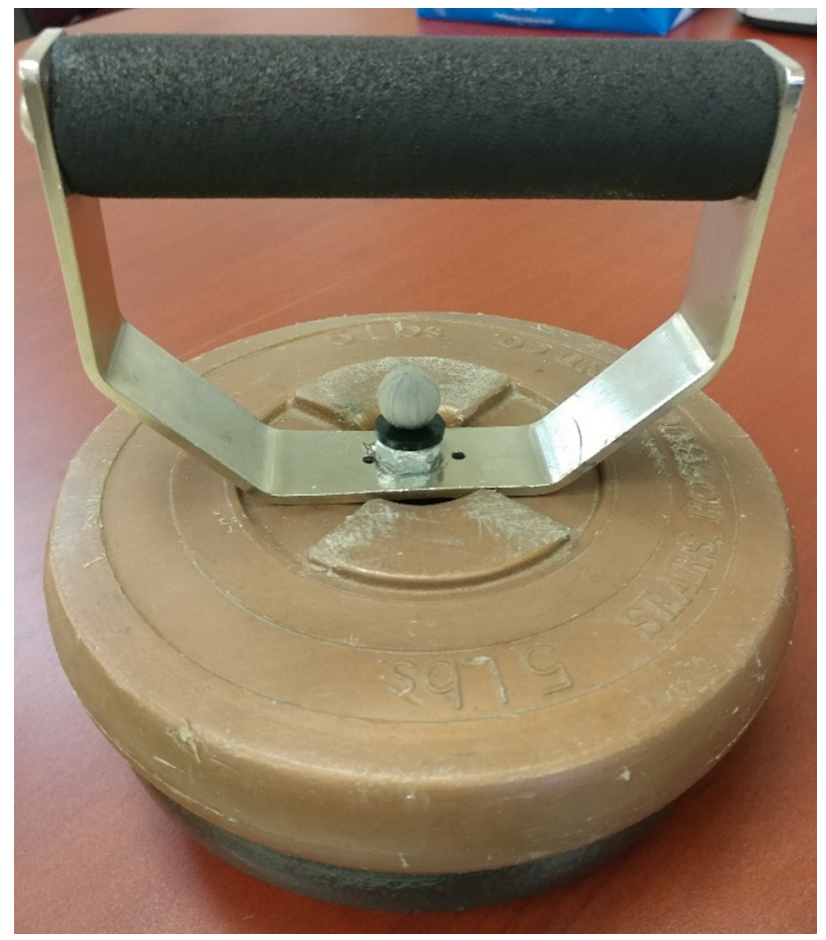

Figure 37. Ten-pound weight with D-handle used as the load during lifting tasks. Retroreflective marker at base of D-handle allows tracking of weight. 
provided in Table 14. Lifting tasks were performed between a) each of the Risk 1 locations (1. High 1 to Medium 1; 2. High 1 to Low 1; 3. Medium 1 to High 1; 4. Medium 1 to Low $1 ; 5$. Medium 1 to High 1, and 6. Medium 1 to Low 1) and between the Risk 1 and Risk 2 locations for (7. High 1 to High 2; 8. High 2 to High 1; 9. Medium 1 to Medium 2; 10. Medium 2 to Medium 1; 11. Low 1 to Low -2; 12. Low 2 to

\begin{tabular}{r|ccc|c}
\hline \multirow{2}{*}{ Location } & \multicolumn{3}{|c|}{ Distances from Sternal Notch (Inches) } & \multirow{2}{*}{ Predicted Strain } \\
\cline { 2 - 3 } & Forward & Right/Left & Up/Down & \\
\hline High 1 & F 20 & 0 & U 10 & 68.9 \\
High 2 & F 10 & L 10 & U 15 & 66.6 \\
Medium 1 & F 10 & R 15 & U 5 & 32.1 \\
Medium 2 & F 5 & L 5 & U 10 & 41.7 \\
Low 1 & F 5 & 0 & D 15 & 4.5 \\
Low 2 & F 5 & R 10 & D 20 & 11.2 \\
\hline
\end{tabular}

Table 14. Distances Forward (F), Right (R) or Left (L) and Up (U) or Down (D) from the sternal notch to the load.

Low 1) for a total of 12 different lifting tasks. Motion capture was used, allowing modeling in AnyBody Modeling System and tracking of the location of the sternal notch, hand, and weight. Modeling in AnyBody was performed to calculate the Composite Index values from the Composite Index selected in Specific Aim 2. Marker coordinates of the sternal notch (CLAV marker) and the Weight (WT marker) were used to predict the shoulder strain using the predictive equations.

\subsection{Participant Consent and Preparation}

Upon arrival, participants were introduced to the experimental procedures and equipment and provided consent with a consent form approved by the local Institutional Review Board (Appendix L). Before beginning data collection, the participant completed the Physical Activity Readiness Questionnaire (PARQ) to access for cardiorespiratory fitness (Appendix C). Answering "No" to any question on the PAR-Q would disqualify the participant from continuing. Participants then removed their shirt and markers were affixed to the participant according to the motion capture marker set described in Section 2.7. A measuring 
tape was used to measure the height of the participant's clavicle, which was used to calculate the tripod heights necessary for each trial. Additionally, the participant was asked to stand on the " $\mathrm{X}$ " where they will be standing for the experimental tasks. The participant was adjusted forward/backward until their sternal notch, i.e. CLAV marker, was directly above the "X" mark. A line was drawn in front of their foot to ensure they stood in the same location during each trial.

\subsection{Experimental Tasks}

Before beginning the experimental trials, the participants were instructed to restrict movement to their right arm, while keeping the trunk still, when performing the lifting tasks. No other instructions or guidance on how to lift and move the load were given. This was done to encourage normal/natural lifting style. For each of the 12 experimental tasks, the tripods were first adjusted to the appropriate heights and placed approximately above the floor marks corresponding to the correct origin and destination locations. A plumb bob (Figure 38) was hung from the bottom center of each tripod and the tripods were precisely aligned, centered above the floor marks.

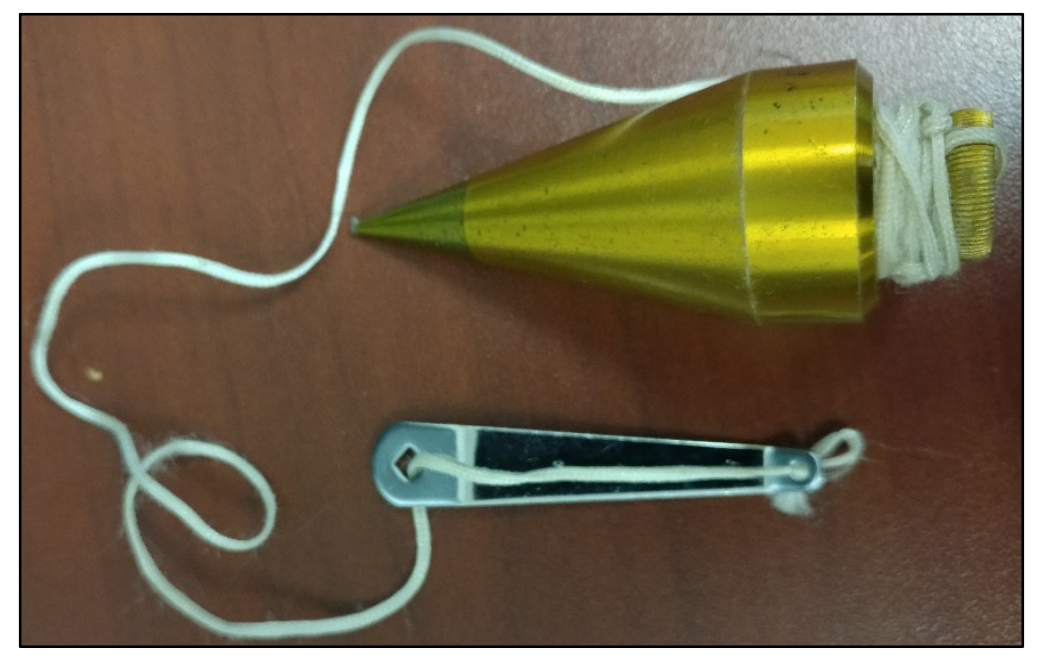

Figure 38. Plumb bob that was hung from the tripod to precisely align the tripods to the proper location. 
After the tripods were appropriately adjusted, the 10-pound weight was placed on the tripod at the origin location. A 10-pound was used in lieu of the 15-pound weight since the weight was unsecured during this portion of the research. The participant stood tall with their hands and arms hanging to their sides with their toes at their line at the "X" mark. When prompted to begin, the participant grasped the D-handle attached to the weights and placed it at the center of the shelf on the tripod at the destination location. After placing the weight at the destination location, the participant returned to the original neutral posture (Figure 39). Two repetitions of each lifting task were performed, for a total of 24 experimental trials. This was done to account for potential failures during modeling and ensure at least one successful trial of each condition existed. Additionally, it allowed the variance between trajectories of the same lift by the same participant to be analyzed. Trial order was randomized. Participants were allowed rest while the tripods were adjusted and moved between locations, which was a minimum of two minutes.

\subsection{Data Processing}

Motion capture data was processed in Vicon Nexus 1.8.1 following the methods described in Section 2.10.1. Marker coordinates of the clavicle, right wrist, right hand and weight markers (CLAV, RWRA, RWRB, RTHB, RFIN, and WT) were exported. Since motion capture was collected during each trial from standing in the neutral posture before the task until after the participant returned to the neutral posture after the lifting task, the actual "lifting phase" had to be extracted from the data. The marker trajectory output of the WT was visually analyzed and timestamps of the beginning and ending of the lifting phase were determined and recorded based on first movement and final movement of the WT marker.

C3D files were exported to be modeled in AnyBody Modeling System using the same musculoskeletal model described in Section 2.1, except using the $4^{\text {th }}$-order Polynomial muscle recruitment strategy, per Section 2.12, since the experimental tasks are dynamic in nature. Of the 120 total trials (5 participants x 24 trials), only 5 failed during modeling. The other repetition for each of these failed trials was successful. The activity of the musculotendon units that comprise the 13 muscles evaluated in Specific Aims 2 and 3 and the Glenohumeral reaction forces were exported to allow further evaluation of the 
predictive equation and calculation of the Composite Index values throughout each trial, respectively. Based on the recorded timestamps of the start and ending points of the lifting phase for each experimental trial, the marker coordinate data (used for calculating the predicted strain) and the Glenohumeral reaction force

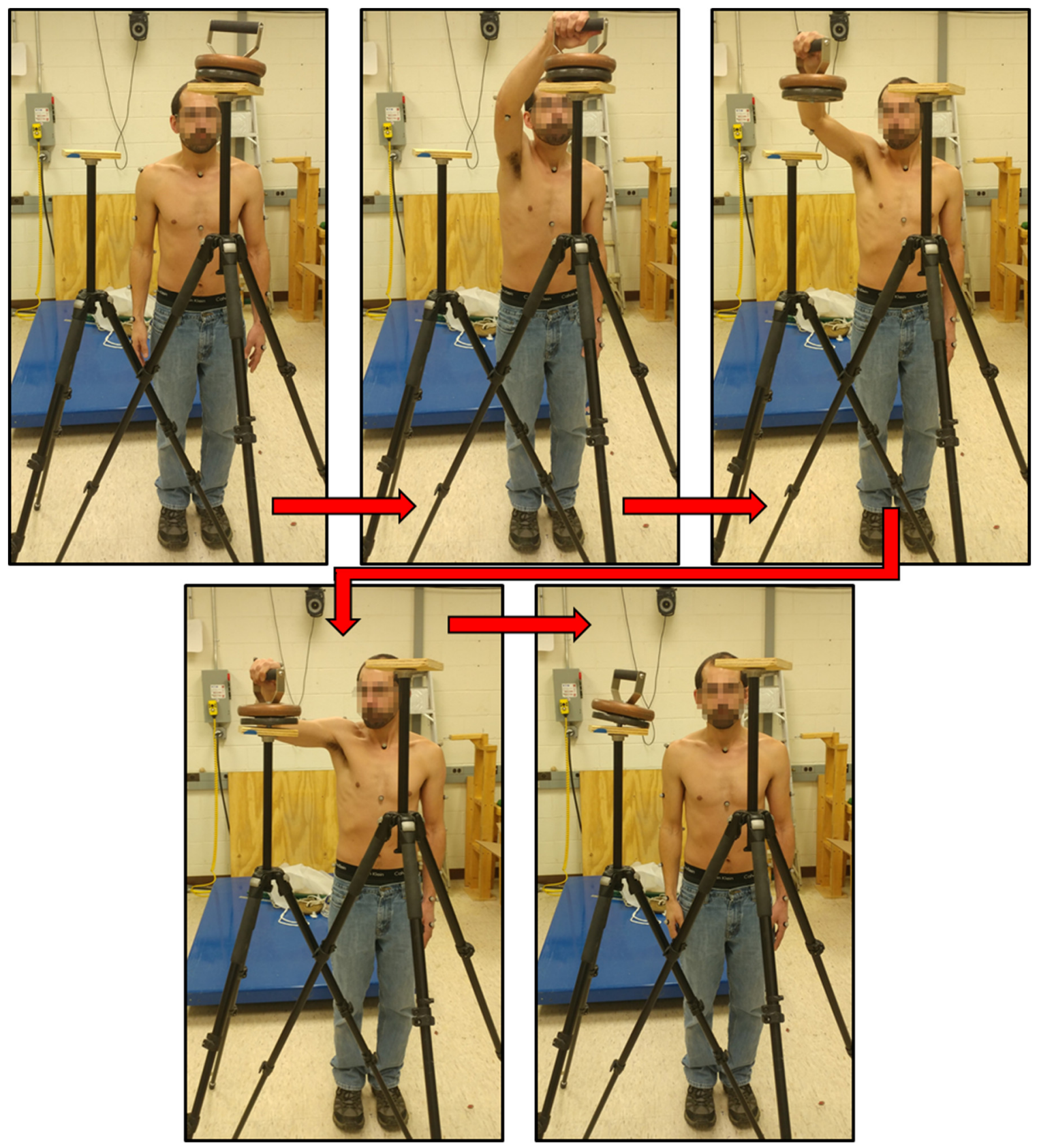

Figure 39. Example progression of an experimental task, showing the participant starting from a neutral position, grasping the weight, placing it onto the destination tripod, and returning to neutral position. 
data (used for calculating the Composite Index) were reduced to include only the lifting phase of the experimental trial.

\subsection{Data Analysis}

Throughout the duration of the lifting phase of each experimental trial, composite index values and predicted strain were calculated. Glenohumeral force output from AnyBody Modeling System was used to calculate the composite index values (Equation 12) for each experimental trial. The marker coordinate data was used to calculate the forward, left/right, and up/down distances from the sternal notch to the load, which was used as input into the predictive equation to calculate the predicted strain. The location of the load was considered palm-center of the hand, calculated as the average of the two right wrist markers (RWRA and RWRB) and the two right hand markers (RFIN and RTHB).

To further validate the predictive equation, correlation and root mean square error analysis were performed between the predicted strain values and the composite index values. Pearson correlation coefficients were calculated using Equation 4 (where $x_{i}=$ predicted strain values, and $y_{i}=$ composite index values) for each experimental trial. Root mean square error values were calculated using Equation 5 (where $\hat{y}=$ predicted strain values and $\mathrm{y}=$ composite index values). When both repetitions of an experimental task by a participant were available (55 of the 60 total tasks - 12 tasks x 5 participants), means of the two correlation coefficients and two RMSE values were shown.

To test the hypothesis that the strain during a lifting task would be the highest at either the origin or destination of the lift, allowing an estimation of the strain across an entire lifting task to be made by calculating the predicted strain at only the origin and destination of the task when performing an unobstructed, one-handed lifting task, the strain at the origin and destination were compared to the maximum strain across the entire lifting task for each of the experimental trials that were successfully modeled with AnyBody Modeling System. 


\subsection{Results}

4.10.1 Further Validation of Predictive Equation: Correlation and root mean square error analysis performed to further validate the predictive equation showed very good support for the predictive equation (Table 15). Correlation coefficients across all trials ranged from $0.608-0.996$, with most correlations above 0.800 (39 out of 60,65\%). Mean correlation coefficients across all 12 trials for each participant ranged from $0.842-0.904$. Root mean square error values ranged from $1.94-15.40$, with most errors below 10 (39 out of 60,65\%). Mean errors across all 12 trials for each participant ranged from $7.077-9.878$. The results from the correlation and root mean square error analysis, applying the predictive equation and composite index equations to a new set of participants in a different set of tasks, suggest that the predictive equation had good accuracy.

Table 15. Mean (of two repetitions) correlation coefficients and root mean square error values between predicted strain values and composite index values for each participant and task.

\begin{tabular}{|c|c|c|c|c|c|c|c|c|c|c|}
\hline \multirow{2}{*}{ Task } & \multicolumn{2}{|c|}{ Participant 1} & \multicolumn{2}{|c|}{ Participant 2} & \multicolumn{2}{|c|}{ Participant 3} & \multicolumn{2}{|c|}{ Participant 4} & \multicolumn{2}{|c|}{ Participant 5} \\
\hline & Correl. & RMSE & Correl. & RMSE & Correl. & RMSE & Correl. & RMSE & Correl. & RMSE \\
\hline High 1 to High 2 & 0.751 & 5.94 & 0.695 & 7.49 & 0.753 & 15.08 & 0.845 & 15.60 & 0.725 & 15.05 \\
\hline High 1 to & & & & & & 7.01 & & 10.21 & & 6.34 \\
\hline High 1 to & 0.707 & 10.01 & 959 & 11.62 & 972 & 5.20 & & 12.18 & & 6.43 \\
\hline High 2 to & 0.712 & 9.21 & 769 & 7.87 & 608 & 12.48 & 0.684 & 12.62 & 0.842 & 17.91 \\
\hline Low 1 to & & & & 10.66 & & 13.65 & & 8.01 & & 6.21 \\
\hline Low 1 tc & 0.806 & 2.58 & 717 & 7.30 & 76 & 3.44 & & 5.66 & & 3.10 \\
\hline & & 7.60 & & 9.2 & & 7.07 & & 6.71 & 986 & 3.58 \\
\hline Low 2 to & 0.848 & 1.94 & 774 & 6.18 & .756 & 2.82 & & 9.83 & 0.749 & 2.76 \\
\hline Med 1 tc & & 8.15 & & 10.4 & 0.988 & 15.40 & & 8.18 & & 7.30 \\
\hline Med $1 \mathrm{t}$ & & & & & & & & 7.40 & & 6.95 \\
\hline Med 1 to & & & & & 0.677 & 13.84 & & 10.32 & & 3.84 \\
\hline Med 2 to Med 1 & 0.737 & 8.47 & 0.614 & 10.84 & 0.775 & 13.08 & 0.856 & 8.83 & 0.988 & 5.46 \\
\hline $\begin{array}{r}\text { Participant } \\
\text { Means: }\end{array}$ & & & & & & & & 9.629 & & 7.077 \\
\hline
\end{tabular}

4.10.2 Origin/Destination Analvsis - Hvpothesis Test: Strain values were stratified into the following risk ranges: Low risk: 0-20; Medium risk: 20-60; High risk: 60-100. Of the 120 collected experimental trials, 115 were successfully modeled in AnyBody Modeling System. Results of the analysis comparing the strain 
at the origin and destination to the peak strain during the experimental task showed some support of the hypothesis (Table 16). Shaded cells in the table were trials where the highest strain occurred at some point other than the origin or destination. Cells were shaded yellow, orange, and red to denote that the maximum strain was $1-2,3-4$, or $5+$ points higher than at the higher between the origin or destination. Of the 115 trials, the max strain was found at the origin or the destination in $53(46.1 \%)$ trials. The remaining 62 (53.9\%) of the 115 trials had strain values higher than the strain at the higher between the origin and destination, not supporting the hypothesis. Of those trials, the max strain was 1-2 points higher (than the

Table 16. Predicted strain values at the Origin (O) and Destination (D) of the lifting task and the max (M) predicted strain across the entire lifting task. Cells highlighted in yellow, orange, and red are trials where the highest strain value did not occur at either the origin or destination in 1-2,3-4, or 5 and more trials, respectively.

\begin{tabular}{|c|c|c|c|c|c|c|c|c|c|c|c|c|c|c|c|c|}
\hline \multirow[t]{2}{*}{ Lifting Task } & \multirow[t]{2}{*}{ Rep. } & \multicolumn{3}{|c|}{$\begin{array}{c}\text { Participant } \\
1\end{array}$} & \multicolumn{3}{|c|}{$\begin{array}{c}\text { Participant } \\
2 \\
\end{array}$} & \multicolumn{3}{|c|}{$\begin{array}{c}\text { Participant } \\
\mathbf{3} \\
\end{array}$} & \multicolumn{3}{|c|}{$\begin{array}{c}\text { Participant } \\
4 \\
\end{array}$} & \multicolumn{3}{|c|}{$\begin{array}{c}\text { Participant } \\
5 \\
\end{array}$} \\
\hline & & 0 & $D$ & $M$ & $O$ & $D$ & $M$ & $O$ & $D$ & $M$ & $O$ & $D$ & $M$ & $O$ & $D$ & $M$ \\
\hline \multirow{2}{*}{ High 1 to High 2} & 1 & 63 & 73 & 76 & 62 & 68 & 70 & 64 & 67 & 72 & 69 & 67 & 79 & 64 & 65 & 75 \\
\hline & 2 & 62 & 67 & 71 & 61 & 68 & 73 & 65 & 67 & 75 & 71 & 67 & 77 & 60 & 65 & 67 \\
\hline \multirow{2}{*}{ High 1 to Low 1} & 1 & 53 & 4 & 53 & 59 & 4 & 59 & 51 & 4 & 51 & 67 & 5 & 67 & 59 & 4 & 59 \\
\hline & 2 & 61 & 4 & 61 & 59 & 4 & 59 & 53 & 4 & 53 & 69 & 5 & 69 & 59 & 4 & 59 \\
\hline \multirow{2}{*}{ High 1 to Med 1} & 1 & 62 & 24 & 62 & 61 & 28 & 61 & 65 & 28 & 66 & - & - & - & 58 & 27 & 58 \\
\hline & 2 & 62 & 24 & 62 & 55 & 28 & 55 & 63 & 28 & 63 & 70 & 29 & 70 & 59 & 28 & 59 \\
\hline \multirow{2}{*}{ High 2 to High 1} & 1 & 69 & 61 & 73 & 72 & 64 & 72 & 69 & 64 & 73 & 69 & 70 & 75 & 66 & 60 & 68 \\
\hline & 2 & 69 & 63 & 75 & 70 & 61 & 73 & 67 & 64 & 72 & 69 & 71 & 74 & 65 & 58 & 65 \\
\hline \multirow{2}{*}{ Low 1 to High 1} & 1 & 4 & 62 & 63 & - & - & - & 4 & 63 & 63 & 5 & 68 & 69 & 4 & 61 & 62 \\
\hline & 2 & 4 & 67 & 67 & 4 & 60 & 60 & 4 & 62 & 62 & 5 & 70 & 70 & - & - & - \\
\hline \multirow{2}{*}{ Low 1 to Low 2} & 1 & 4 & 11 & 11 & 4 & 11 & 12 & 4 & 11 & 14 & 5 & 12 & 14 & 6 & 12 & 12 \\
\hline & 2 & 4 & 11 & 12 & 5 & 11 & 12 & 4 & 12 & 13 & 4 & 12 & 14 & 6 & 11 & 12 \\
\hline \multirow{2}{*}{ Low 1 to Med 1} & 1 & 4 & 26 & 26 & 4 & 28 & 32 & 4 & 28 & 32 & 4 & 28 & 41 & 4 & 26 & 29 \\
\hline & 2 & 4 & 25 & 26 & 5 & 29 & 32 & 5 & 28 & 32 & 5 & 29 & 38 & 6 & 26 & 27 \\
\hline \multirow{2}{*}{ Low 2 to Low 1} & 1 & 11 & 4 & 11 & 11 & 5 & 11 & 11 & 4 & 11 & 13 & 5 & 13 & 13 & 6 & 13 \\
\hline & 2 & 11 & 4 & 11 & 12 & 4 & 12 & 12 & 4 & 12 & 13 & 5 & 18 & 11 & 6 & 11 \\
\hline \multirow{2}{*}{ Med 1 to High 1} & 1 & 25 & 60 & 60 & 29 & 61 & 62 & 29 & 62 & 62 & 30 & 68 & 68 & 28 & 60 & 60 \\
\hline & 2 & 25 & 61 & 61 & 28 & 60 & 60 & 28 & 63 & 63 & 31 & 68 & 69 & 29 & 60 & 61 \\
\hline \multirow{2}{*}{ Med 1 to Low 1} & 1 & 27 & 4 & 29 & 29 & 4 & 29 & 28 & 4 & 30 & 28 & 5 & 37 & 27 & 4 & 29 \\
\hline & 2 & 27 & 4 & 28 & 29 & 4 & 32 & 28 & 5 & 28 & 29 & 5 & 32 & 26 & 5 & 26 \\
\hline \multirow{2}{*}{ Med 1 to Med 2} & 1 & 27 & 36 & 39 & - & - & - & 29 & 37 & 41 & 29 & 38 & 46 & 27 & 42 & 44 \\
\hline & 2 & 27 & 35 & 39 & 29 & 40 & 42 & 30 & 38 & 41 & 29 & 38 & 46 & 28 & 42 & 45 \\
\hline \multirow{2}{*}{ Med 2 to Med 1} & 1 & 35 & 27 & 35 & 39 & 28 & 39 & 40 & 29 & 40 & 39 & 29 & 45 & 35 & 27 & 35 \\
\hline & 2 & 36 & 27 & 40 & 39 & 27 & 39 & - & - & - & 39 & 29 & 45 & 41 & 26 & 41 \\
\hline Legenu. & \multicolumn{5}{|c|}{$\begin{array}{c}\text { Max 1-2 higher than } \\
\text { O/D }\end{array}$} & \multicolumn{5}{|c|}{$\begin{array}{c}\text { Max 3-4 higher than } \\
\text { O/D }\end{array}$} & \multicolumn{5}{|c|}{$\begin{array}{c}\text { Max 5+ higher than } \\
\text { O/D }\end{array}$} & \\
\hline
\end{tabular}


higher of the origin and destination) in $25(40.3 \%)$ trials, $3-4$ points higher in $20(32.3 \%)$ trials, and 5 or more points higher in $17(27.4 \%)$ trials. The mean difference across all trials was 2.8 points, while the mean difference across only trials with a non-zero difference was 5.1 points. The highest difference found was 13 points (Participant 4 - Low 1 to Medium 1, Rep 1). However, none of the trials would be re-classified into a different risk category (High, Medium, Low) due to these differences.

Participant 4 had more and higher differences than the remaining 4 participants (Table 17). Without Participant 4, the highest difference would have been 10 points, occurring only once (Participant 5 - High 1 to High 2, Rep 1). This suggests that there was something inherently different about the manner in which Participant 4 lifted.

Table 17. Breakdown for each participant of number of different trials, where the strain was highest at some point other than the origin or destination, and the mean differences across all trials and only the different trials.

\begin{tabular}{cccc}
\hline Participant & \# Different (\%) & Mean Difference (All) & $\begin{array}{c}\text { Mean Difference } \\
\text { (Non-Zero) }\end{array}$ \\
\hline $\mathbf{1}$ & 12 of $24(50.0 \%)$ & 1.4 & 2.8 \\
$\mathbf{2}$ & 10 of $22(45.5 \%)$ & 1.1 & 2.5 \\
$\mathbf{3}$ & 12 of $23(52.2 \%)$ & 1.9 & 3.7 \\
$\mathbf{4}$ & 17 of 23 (73.9\%) & 4.2 & 5.7 \\
$\mathbf{5}$ & 11 of 23(47.8\%) & 1.2 & 2.5 \\
\hline
\end{tabular}

\subsection{Discussion}

4.11.1 Further Validation of Predictive Equation: The results of the correlation and root mean square error analysis between the composite index values and the predicted strain values from the experimental trials showed a great deal of support for our predictive equation. Overall, the correlation values were high. The root mean square error values, which were often in double digits, were not surprising, and were likely due to two primary factors. First, Since the load used during the experimental tasks in this Specific Aim was only 10-pound while the predicted equation was derived based on experimental tasks using a 15-pound 
load, this decreased the composite index values compared to the predicted values. Additionally, the anthropometrics of the AnyBody model, including musculotendon unit cross-sectional area, were scaled based on the height and weight of the participant. Since the reference values used in the composite index calculations were taken at the point where the model became overloaded, the reference values increase as the cross-sectional area of the musculotendon units for the participant are higher, which would result in a decrease in the composite index values.

Plots of the strain from one repetition per participant of the most highly correlated tasks, Low 1 to High 1 (Figure 40) and Low 1 to Medium 1 (Figure 41), provide further evidence of the accuracy of the predictive equation. In tasks with lower correlations, such as High 1 to High 2 (Figure 42), the results are still reasonable, although there is higher variation and less consistency in the composite index values across the trials. The composite index values nearing and at the destination of this lifting task were much lower than predicted, with differences of up to 35 , for 4 of 5 participants. Compared to the opposing trial, High 2 to High 1 (Figure 43), the composite index values for each location (High 1 and High 2) differed less from the predicted strain values when the location was the origin of the lift than when it is the destination.

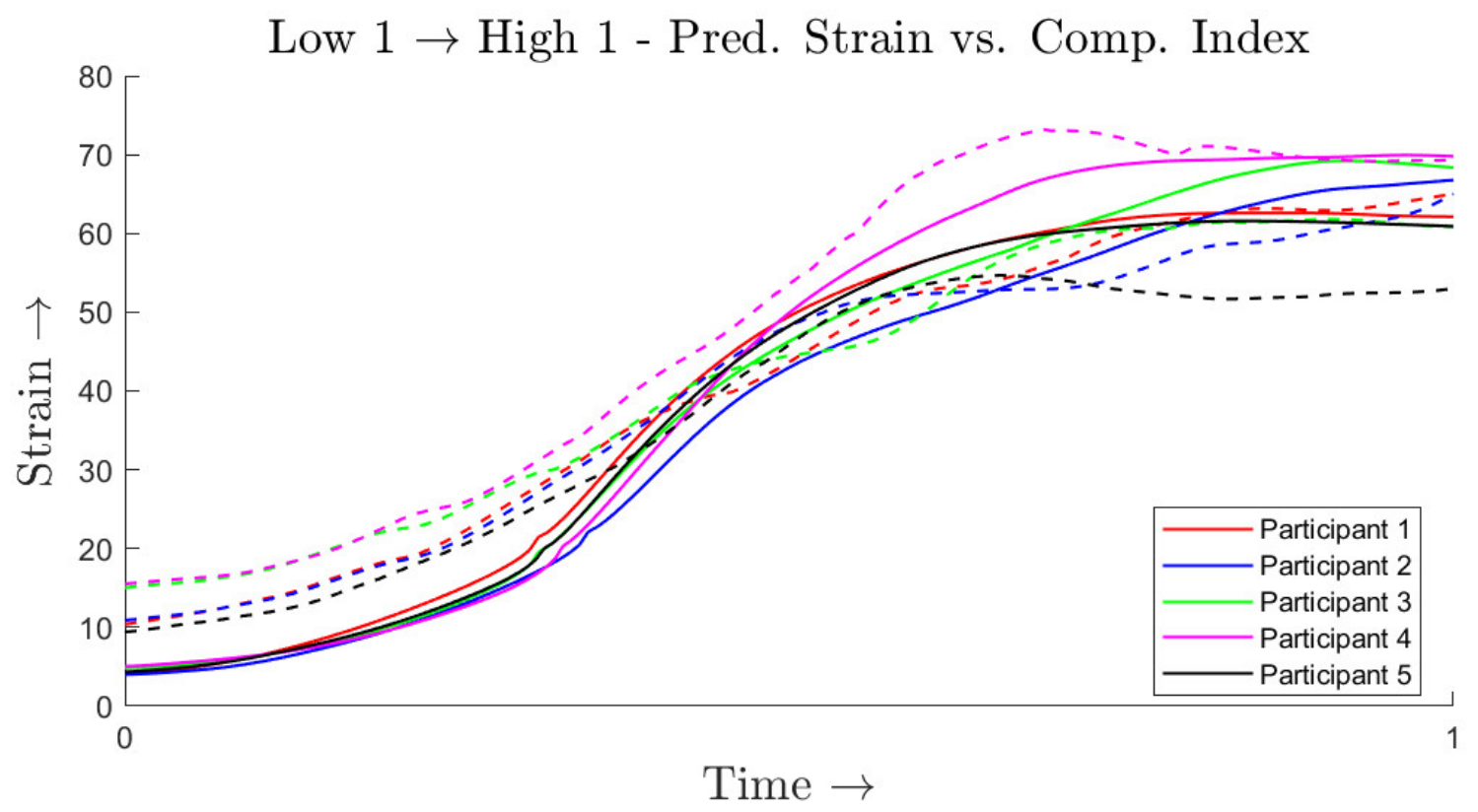

Figure 40. Predicted strain (solid line) and composite index (dashed line) for Low 1 to High 1 task. 


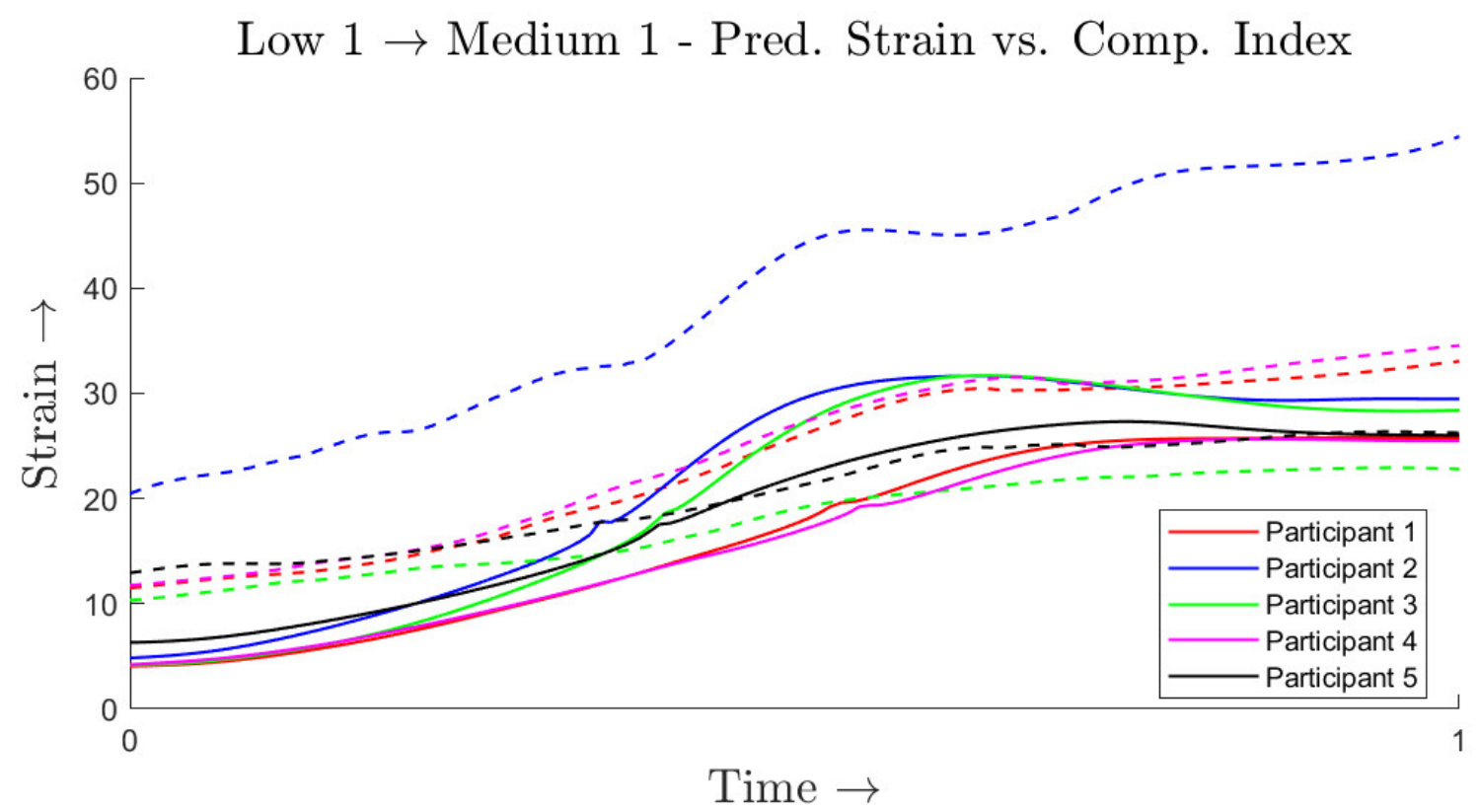

Figure 41. Predicted strain (solid line) and composite index (dashed line) for Low 1 to Medium 1 task.

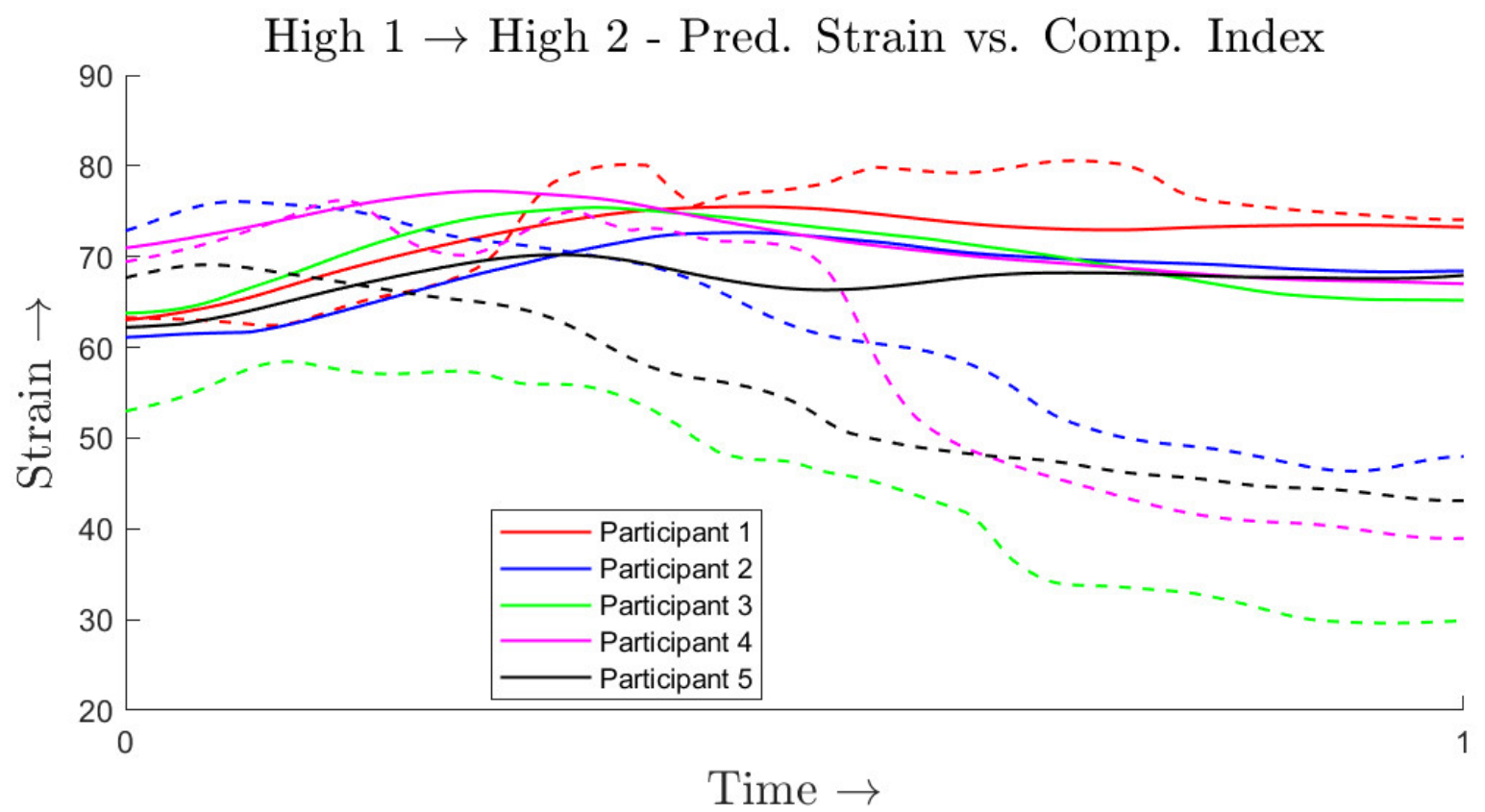

Figure 42. Predicted strain (solid line) and composite index (dashed line) for High 1 to High 2 task. 
Therefore, some other factor/characteristic of the lift must be influencing the composite index values differently between the beginning and end of the lifting task. However, this characteristic is less- or non-existent in the Low and Medium risk zones. This characteristic, along with the less consistent changes and sporadic increasing and decreasing of the composite index values that can be seen in the High 1 to High 2 and High 2 to High 1 tasks, especially during the first half of the lifting task, could be a result of the algorithmic nature of the muscle activation computation.

Plots of the three components of the Glenohumeral reaction force (Figure 44) and the musculotendon unit activations (Figure 45) and from Participant 2 performing the High 2 to High 1 and Low 1 to Medium 1 lifting tasks demonstrate that the Glenohumeral reaction force was "unstable" when musculotendon units in the AnyBody model begin reaching their maximum exertion. In the model, when a muscle that is predominantly responsible for an action, e.g. the deltoid with shoulder abduction, reaches the model- calculated maximum exertion, other musculotendon units with potentially less mechanically

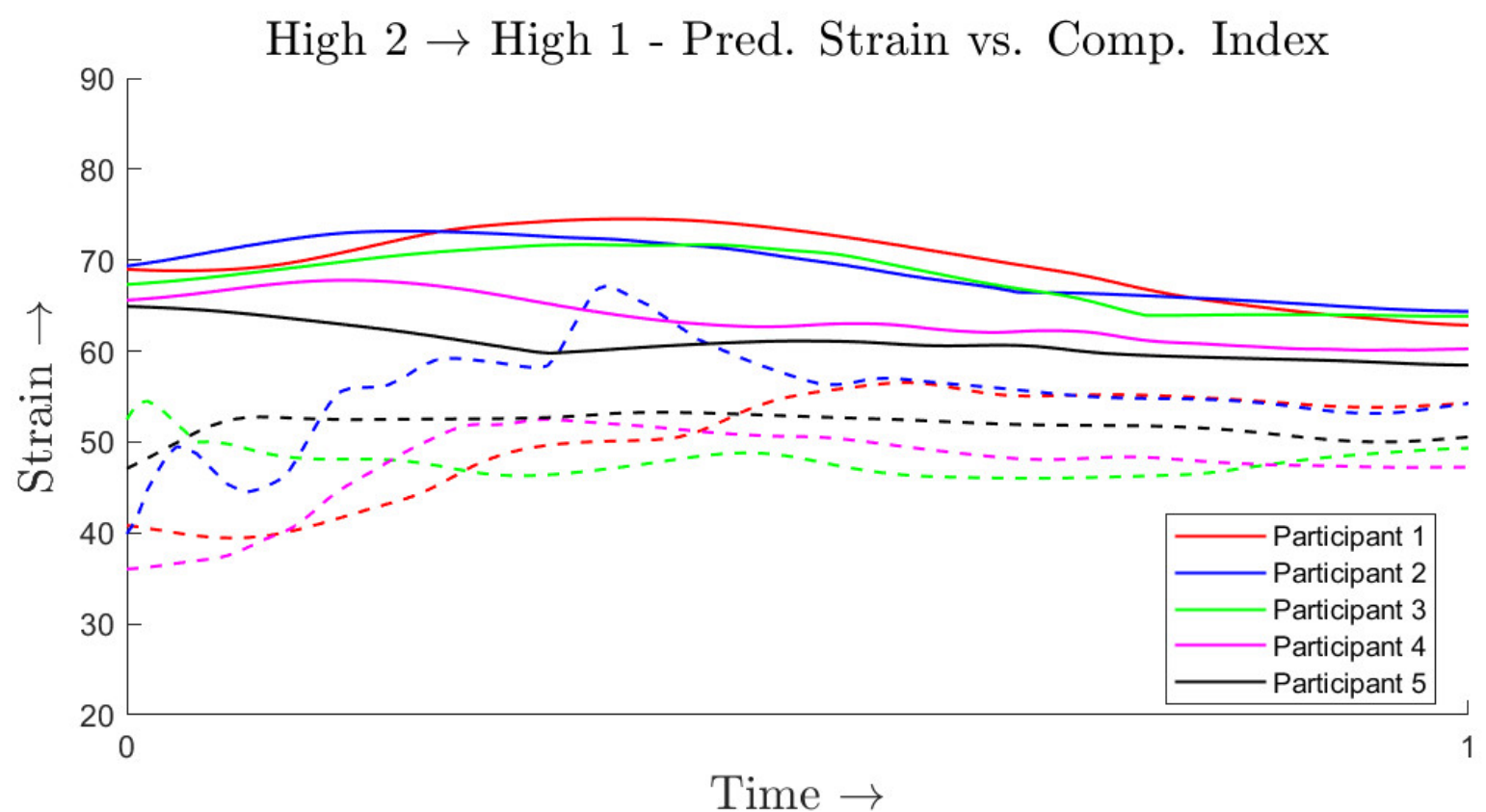

Figure 43. Predicted strain (solid line) and composite index (dashed line) for High 2 to High 1 task. 
advantageous lines of action must compensate. This "muscle swapping" caused sudden deviation in the overall magnitude and direction of the Glenohumeral reaction force. However, when muscle swapping naturally occured throughout the lift based on changes in posture, as seen in the Low 1 to Medium 1 muscle activation plots, the changes were more gradual, and therefore did not result in instability in the Glenohumeral reaction forces.

By default, the model used in this research relied on a single musculotendon strength constant multiplied by the cross-sectional area of the musculotendon units, which are scaled based on height and weight of the modelled participant. Due to the programmatic nature of AnyBody, the musculotendon strength constant in AnyBody modeling system can be modified and it would even be possible to use different constant values for different musculotendon units. Therefore, it is theoretically possible to avoid the musculotendon units being maximally exerted by manipulating the constant(s). However, this would not necessarily result in an increase in accuracy and could reduce overall accuracy unless done in a reasoned and systematic manner. If the constant(s) are increased too much, it could demand a smaller muscle with a more mechanically
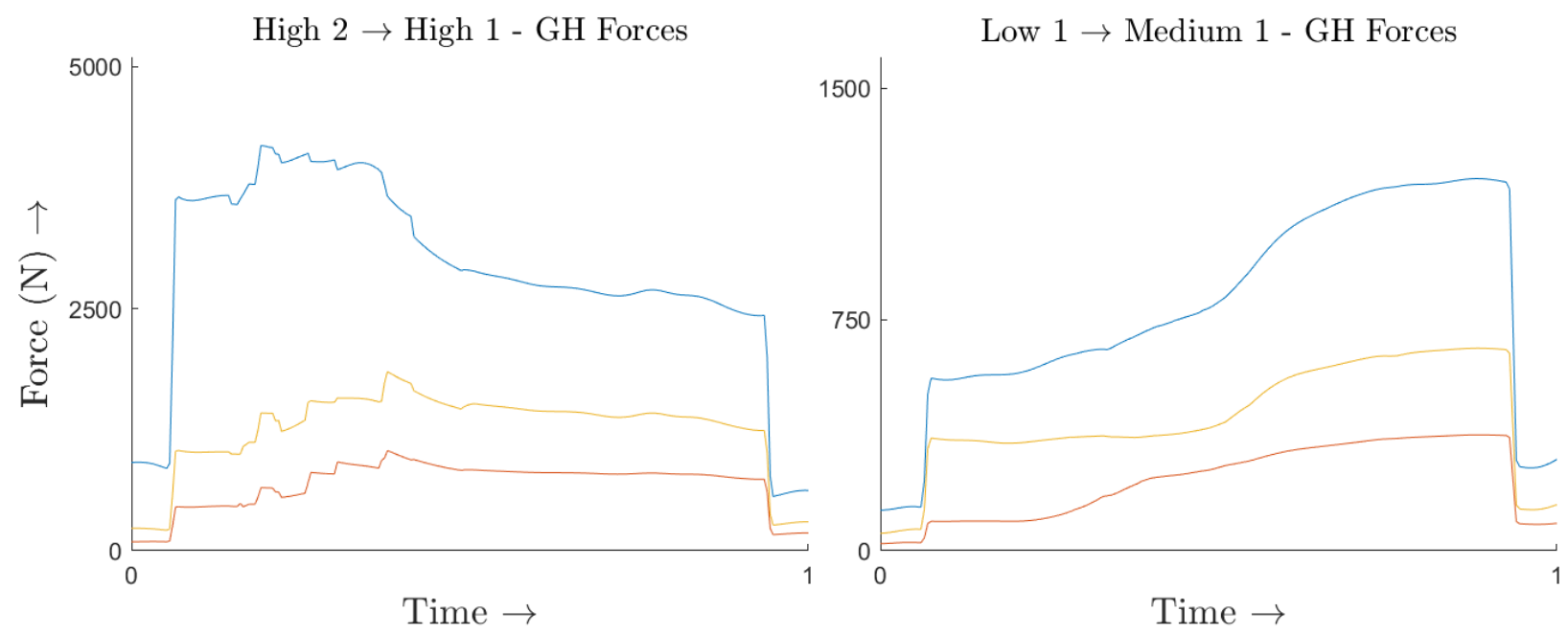

Figure 44. Glenohumeral force components for Participant 2 performing the High 2 to High 1 and Low 1 to Medium 1 tasks. 

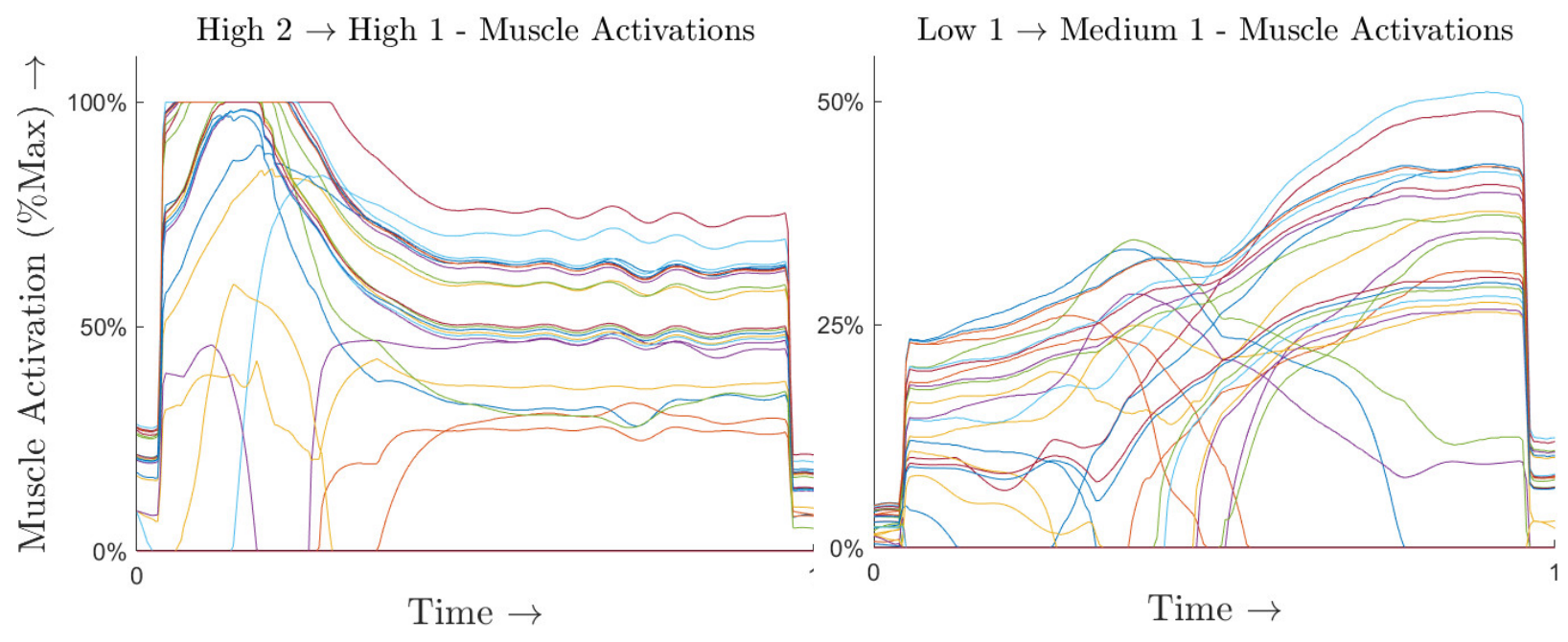

Figure 45. Musculotendon activations for Participant 2 performing the High 2 to High 1 and Low 1 to Medium 1 tasks.

advantageous line of action to perform a much higher exertion than realistically possible. Therefore, whichever values for the constants were selected would have to be based on some evidence, and a calibration process would have to be performed from the initial steps of the research to ensure consistency.

This could, theoretically, be achieved by having the participant perform one maximal exertion (or one exertion per constant if multiple constants are used). If only one constant is desired, the constant could be optimized by having each participant perform a maximal exertion, such as a lifting exertion with the arm in a specific posture, while using a dynamometer to measure the amount of force generated during the exertion. Next, the exertion could be modeled multiple times with the generated force applied, manipulating the strength constant between executions until a muscle in the model is maximally exerted. If multiple constants were desired, the same process could potentially be used to determine optimized constants for specific muscles. However, this is untested and may not work as expected due to the algorithmic nature of the computation of the musculotendon unit activations. 
4.11.2 Origin/Destination Analysis - Hypothesis Test: The results of the origin/destination strain analysis performed to test the hypothesis that the strain would be the highest at either the origin or the destination of the lifting task did not support the hypothesis. In 62 of the 115 experimental trials, the peak strain was found somewhere other than the origin or the destination. However, of the remaining 53 trials, $36(72.6 \%)$ were within 4 points of the higher of the origin or destination, and a disproportionate amount (11 of 17) with an error of $5+$ were isolated to Participant 4, which means there may still be some validity to assuming the strain of the task based on the strain at the origin/destination, but emphasizes the variations possible in lifting technique

Visual analysis of several experimental tasks was performed to determine why the hypothesis was not supported and try to identify any characteristics among the lifting trajectories. High 1 to High 2 was selected because it had the most differences of 5+ points. Figure 46a-c show plots of the trajectories of the
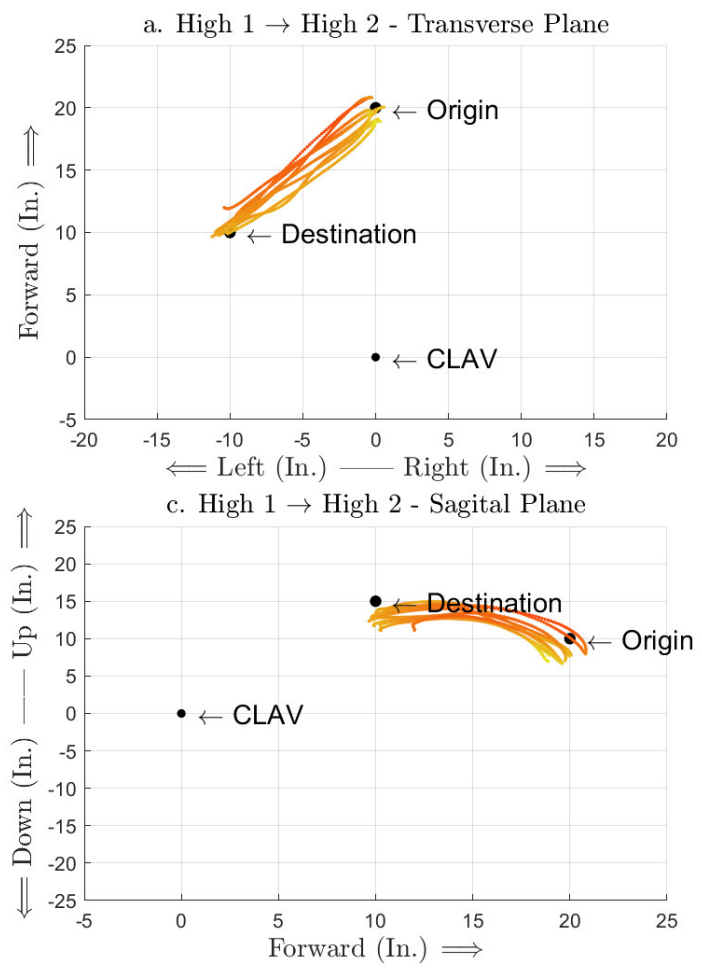
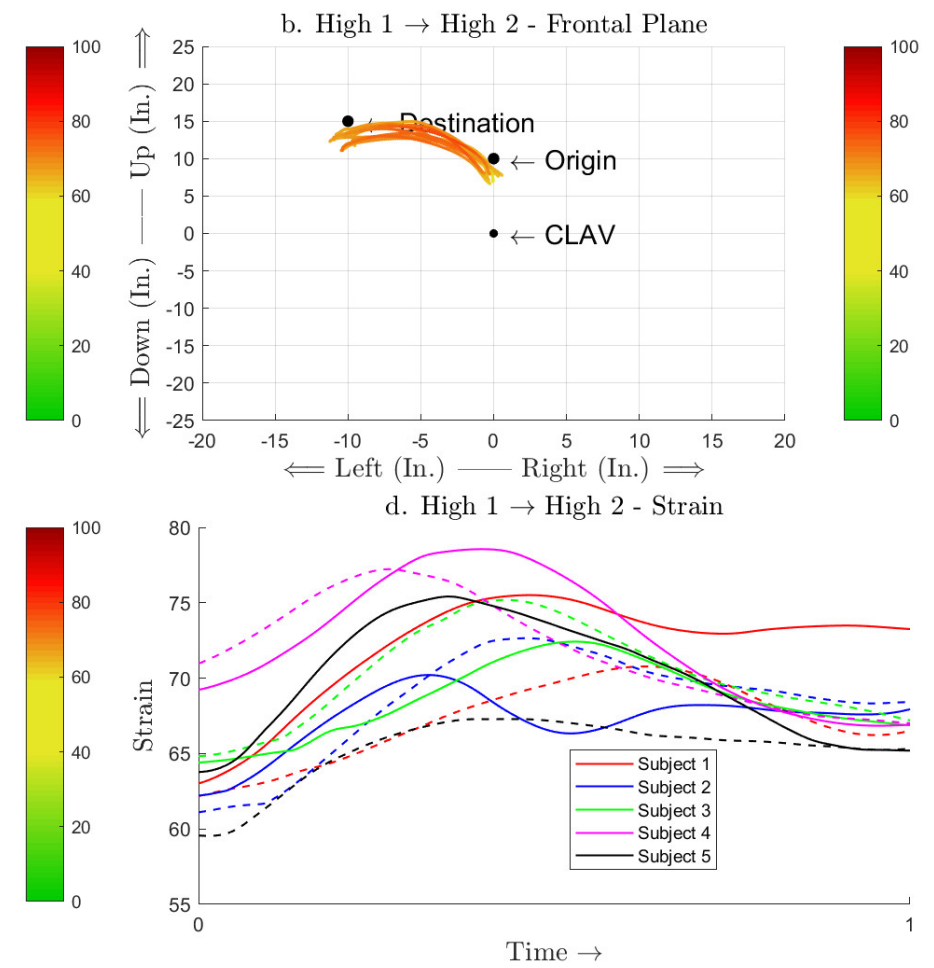

d. High $1 \rightarrow$ High 2 - Strain

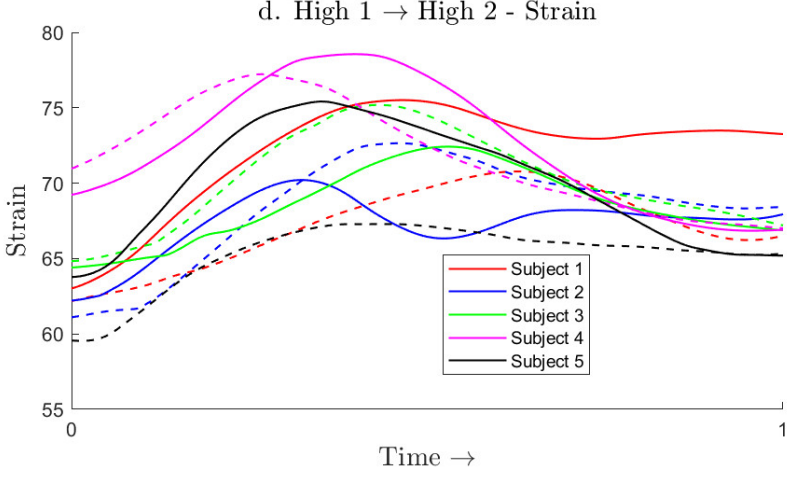

Figure 46. Trajectory and strain plots for the High 1 to High 2 experimental task. a-c) Trajectories of the lift from the origin to the destination in the transverse, frontal, and sagittal planes with the markers colorcoded according to the strain. d) Plot of strain throughout the duration of the lifting tasks, with solid lines and dashed lines indicating repetitions 1 and 2, respectively. 
load for each of the 10 trials, color-coded based on the strain at that point, in each of the anatomical planes while Figure 46d shows the predicted strain throughout each of the 10 trials. Trials from each participant are a different color with solid and dashed lines indicating repetitions 1 and 2 , respectively. The spike in the strain during the lift occurred within the first half of the lift. Figure 46b-c shows that one potential reason that the strain is highest somewhere other than an endpoint of the lift is that the trajectory arcs between the two locations, with an initial lifting from the origin, causing the trajectory to cross an area of higher strain. If the trajectory of the lift formed a straight line between the two locations, it is likely that the hypothesis would hold true. When looking at another task with higher differences, Low 1 to Medium 1 (Figure 47), similar characteristics existed, except surrounding the destination portion of the lifting task; the participant moved the item above the destination and lowered it onto the shelf. A few of the experimental tasks, however, were in support of our hypothesis, including High 1 to Low 1, High 1 to Medium 1, and Low 2 to Low 1. Similar plots for High 1 to Low 1 (Figure 48) were developed and showed
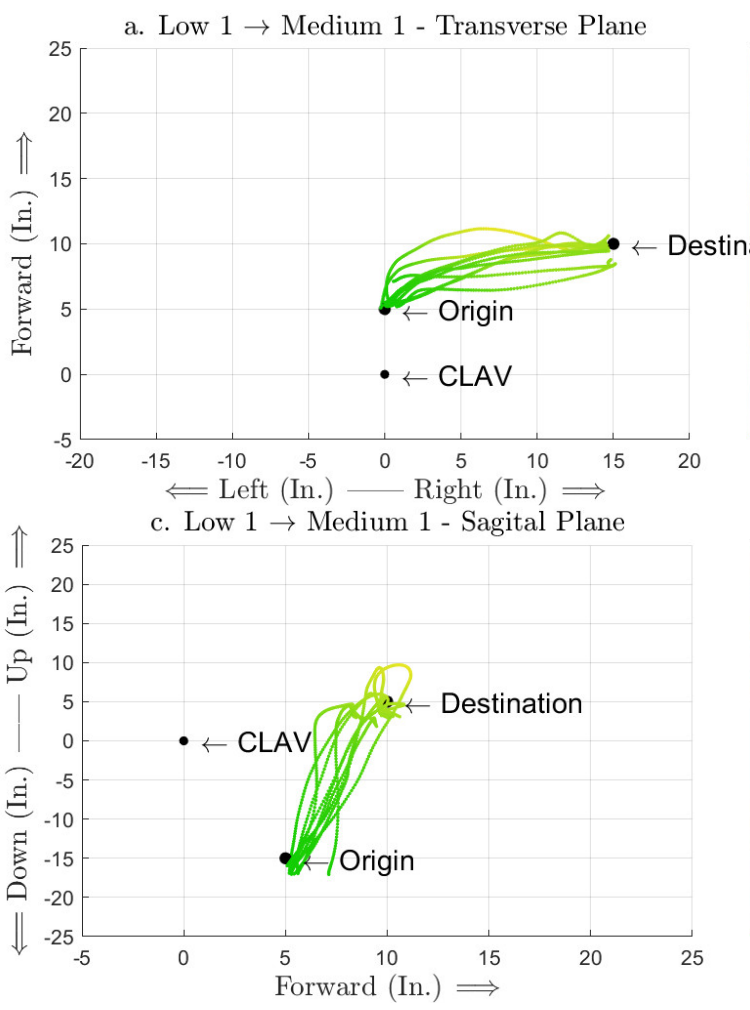

b. Low $1 \rightarrow$ Medium 1 - Frontal Plane

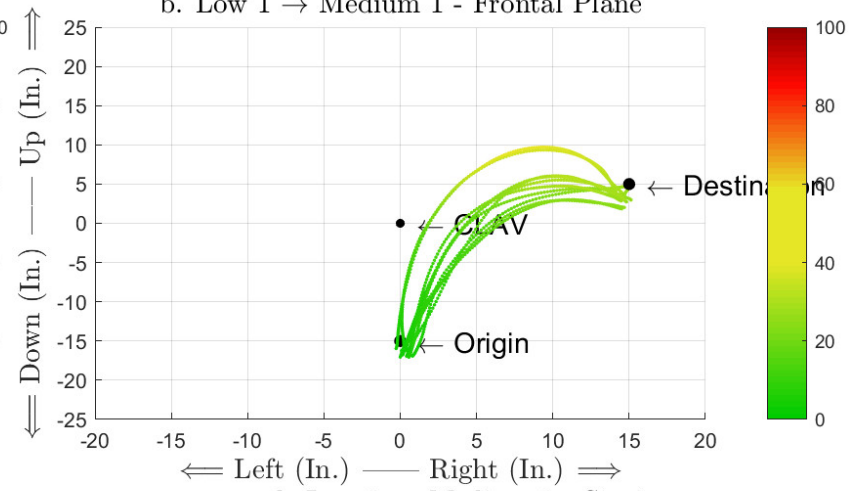

d. Low $1 \rightarrow$ Medium 1 - Strain

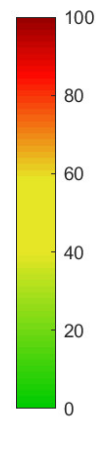

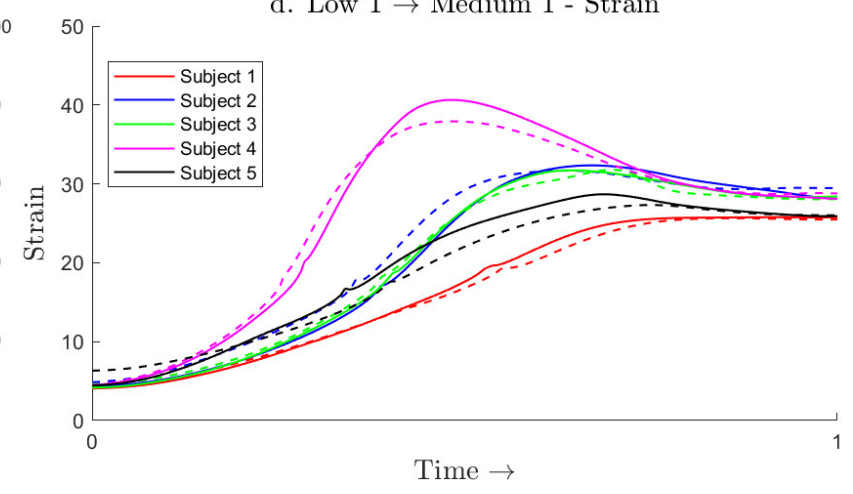

Figure 47. Trajectory and strain plots for the Low 1 to Medium 1 experimental task. 

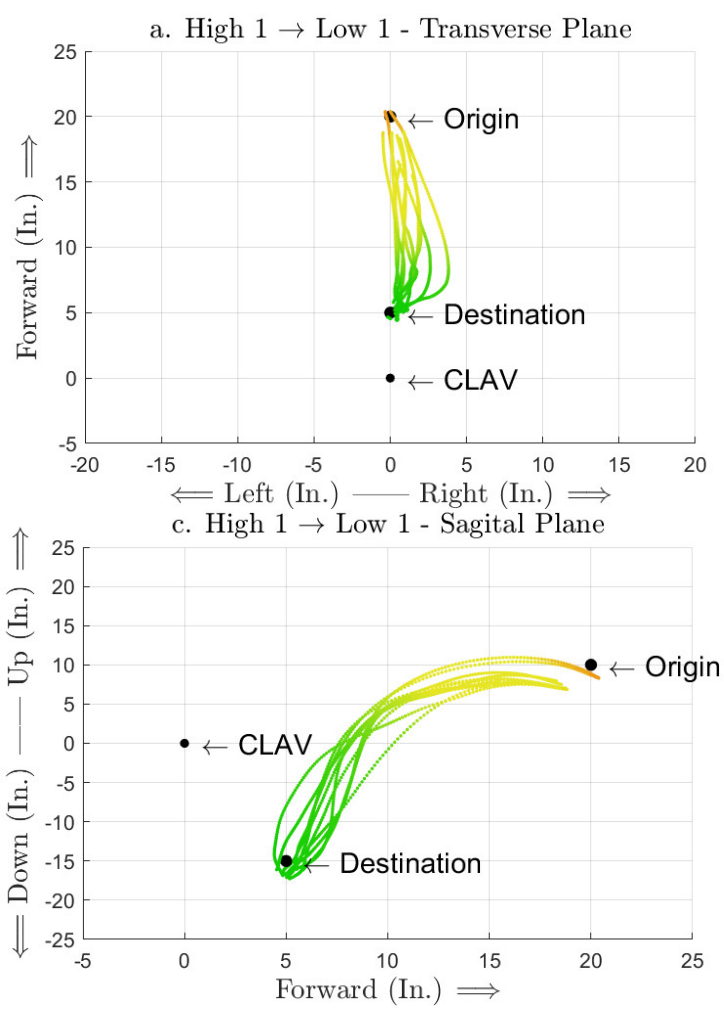

b. High $1 \rightarrow$ Low 1 - Frontal Plane
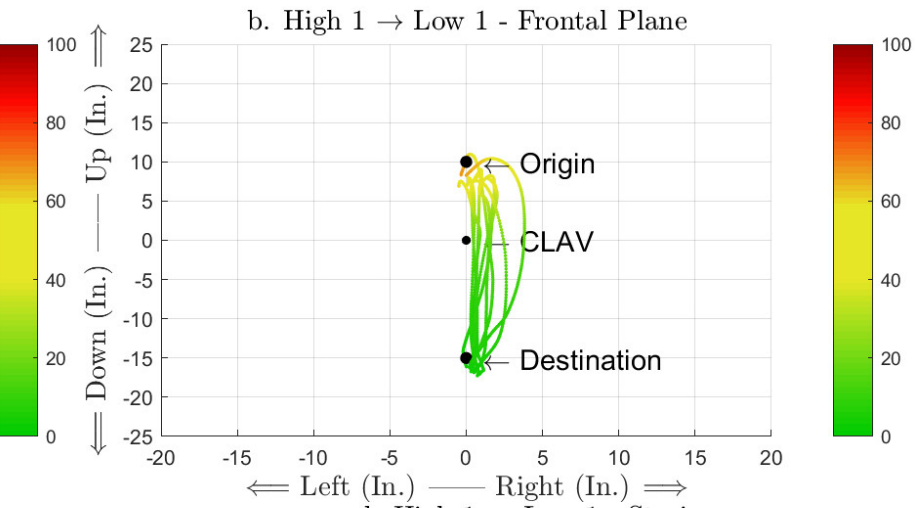

d. High $1 \rightarrow$ Low 1 - Strain

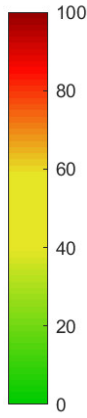

Figure 48. Trajectory and strain plots for the High 1 to Low 1 experimental task.

that, for this task, while the trajectory of the lift was still an arc, it began with a more horizontal trajectory instead of an initial lifting phase.

Finally, when looking at a task that had a mixture of supporting and opposing trials, Medium 1 to High 1 (Figure 49), the data shows the same arcing characteristic of the trajectory, but the lifting trajectory tended to end with a more horizontal trajectory. In each of the 3 trials in which the strain was highest somewhere other than the destination or origin, the difference was only 1-2 points.

Therefore, while the strain was not always highest at the origin or destination, the differences between the peak strain and the strain at the higher of the origin or destination were, generally, small. With some consideration to the potential for an initial lifting motion from the origin of the lift, this tool could still be useful for analysis of a lifting task because, ultimately, the approximate strain of the task will be largely dictated by the strain at the higher of the origin/destination. Ultimately, the peak strain was generally 
within 5 points of the higher between the origin and the destination, and therefore, with this in mind, the origin/destination analysis may still be feasible.

a. Medium $1 \rightarrow$ High 1 - Transverse Plane

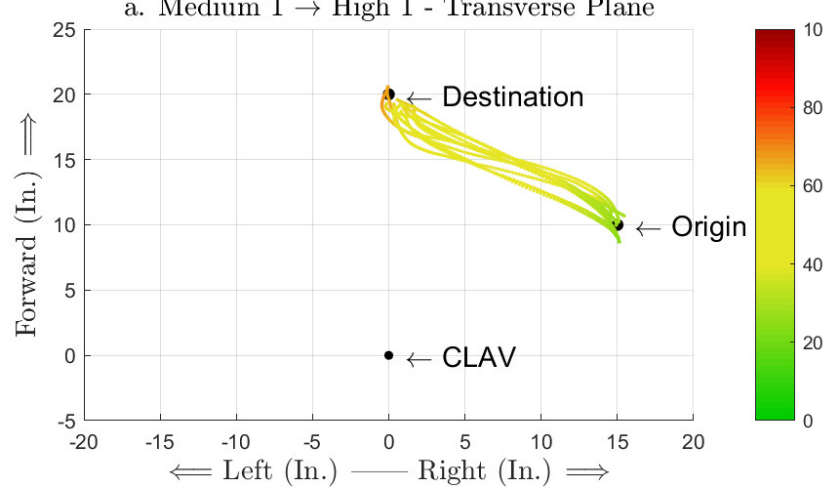

c. Medium $1 \rightarrow$ High 1 - Sagital Plane

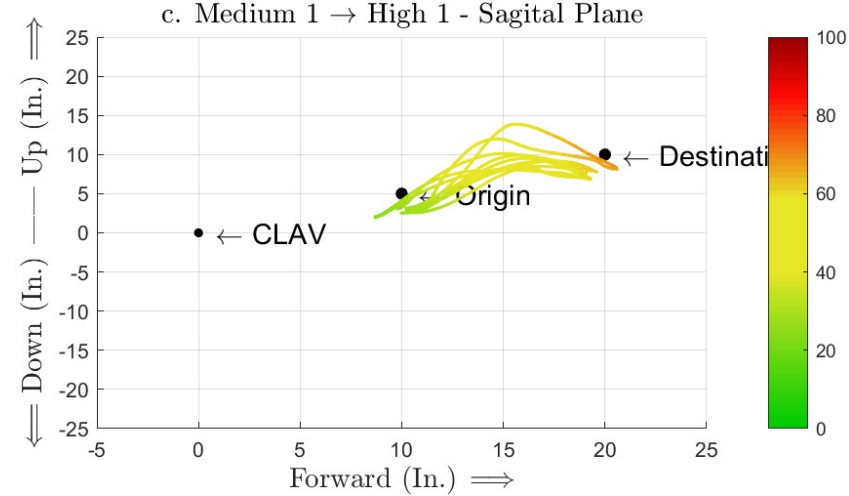

b. Medium $1 \rightarrow$ High 1 - Frontal Plane

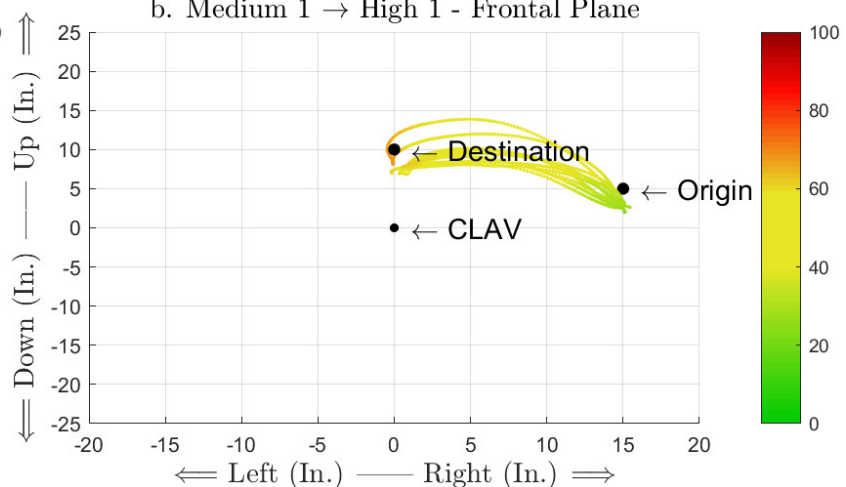

d. Medium $1 \rightarrow$ High 1 - Strain

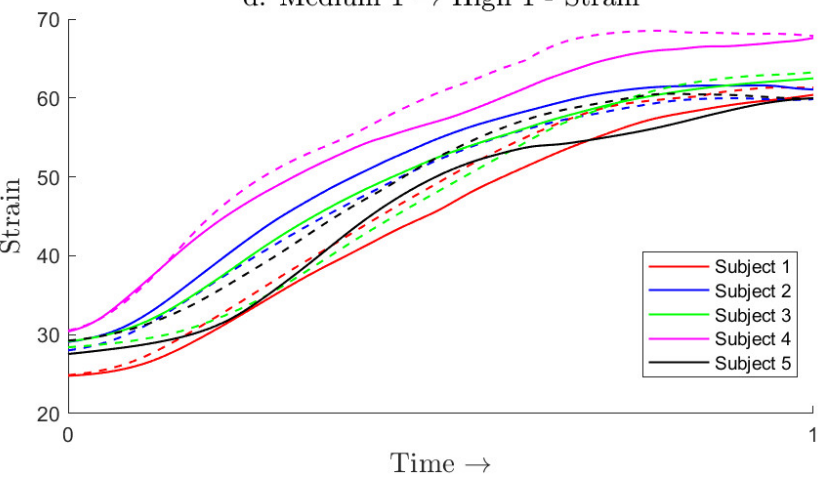

Figure 49. Trajectory and strain plots for the Medium 1 to High 1 experimental task. 


\section{DISCUSSION}

AnyBody Modeling System is a powerful and flexible tool. Due to the flexibility, the quality of the results are dependent on the quality of the input data and the model itself. In this research, the first specific aim involved selecting the most appropriate muscle recruitment strategy for the modeled tasks by comparing electromyography results to the muscle activations from the corresponding muscles. However, there are other steps that could be taken to further improve the results when resources allow, such as calibration of the muscle strength constants. However, no matter how good the input, the muscle activations are still being determined by an algorithm, which cannot precisely replicate the actual muscle activations of the participants. Regardless, this is the best method currently available to estimate the forces generated within the body, because AnyBody Modeling system and the open-source alternative OpenSim, are the only software/methods that consider the forces generated due to the tension of the muscles AND the forces/moments generated due to the mass and accelerations of the segments and load, instead of just the latter.

This research had several limitations; first with the sample size and selection. This was not a funded project and therefore resources were very limited. Therefore, participant recruitment was a convenience sample, primarily among the student body at West Virginia University, and the sample sizes were limited. The participants were not skilled workers and were typically younger, which is not representative of the working populations where an analysis tool such as this would be used. It would not be wise to extrapolate these findings to experienced workers that cross wider demographical ranges.

Additionally, only male participants were recruited. This was done mostly out of necessity. Electromyography sensor and motion capture marker placements on the trunk would not have been appropriate for female participants. Additionally, the weights used in this research may be difficult for some females to lift, especially in awkward/extended postures. Therefore, the analysis would have to be performed either with lower weights for the females only or lower weights for all participants. If lower weights were used for all participants, males would often not be near maximal exertion. If lower weights 
were used for females only, separate analysis would have to be performed for each gender, possibly throughout the entire research.

Another limitation was the lack of ability to precisely control the location of the participant, or more importantly their sternal notch. In the second specific aim, the load was hanging from a rope with only its height adjusted, while the participant moved around the grid to the appropriate locations. The participants' locations were controlled by having them stand with their feet in certain positions. In the third specific aim, the participant stood in one location and the load was adjusted by moving and adjusting the tripods. In both cases, the participants were instructed to stand straight, but their upper body posture was otherwise uncontrolled. Therefore, there was some variation between the intended location and actual location of the sternal notch during the experimental trials. It may have been possible to restrict the participants' motion further, but since that level of precision of the load location was not critical for this research, it was decided to avoid unnecessary restrictions on motion that may reduce real-world applicability. However, that may have been done, anyway, by requiring the upper body kept straight. For instance, task locations requiring reaching across the body would typically be done with some twisting of the torso, and by restricting the motion, the lifting posture that was used may not occur naturally. In hindsight, it may have been better to allow the participant to move their upper body, but that would require some additional considerations. For instance, using the sternal notch as a reference point would not be as straightforward, but may still be possible by using the position of the sternal notch when the participant is standing upright in a neutral posture before reaching for the load to represent the position of the sternal notch.

AnyBody Modeling System, while a very powerful software system, has several limitations that became more apparent as this research progressed. During static motions, the musculotendon unit activity from the models is logical. When a static posture is maintained, the same musculotendon units remain active at similar levels with little to no muscle switching, i.e. one musculotendon unit de-activating and another musculotendon unit activating to take over. During dynamic tasks, the algorithm-driven musculotendon 
unit activations and changes in posture throughout the tasks results in muscle-switching in the model that are not physiologically accurate representations of what happens in the body. The capabilities and accuracy of the AnyBody Modeling System would benefit by allowing input of, at least, electromyography signals, but ideally electromyography signals from maximum voluntary contractions, as well. This could supplement or replace the algorithms to help improve the musculotendon unit activation predictions of the model by better matching muscle activity to musculotendon unit activity. This would improve the accuracy of the reaction force estimates and eliminate one of the major limitations of the AnyBody Modeling System.

While not a limitation, future research of this type utilizing AnyBody Modeling System would be prudent to focus more effort on modifying the base model more accurately for each participant, including anthropometric scaling, strength scaling, and marker placement, even if it necessitated reducing the number of participants. In this research, the models were scaled based on height and weight, and while this may result in a close representation of the participant, there are no "scaling factors" that are accurate for everyone. Therefore, the anthropometry of the model is likely to differ in some way, potentially significant to the results. Using anthropometric equipment to take measurements of the participants and using actual dimensions within the software could result in more accurate representation of the movement and fewer failures during modeling. By default, AnyBody Modeling System models use a strength scaling factor that is multiplied by the cross-section of the musculotendon units to determine their maximum strength capability. Inaccuracies in the strength scaling could lead to inappropriate musculotendon unit activation and, in cases where the strength scaling factor is too low, modeling failures due to overloaded muscle configurations. In this research, the default value was used. One avenue toward improving the strength scaling of the model would be to record force exertion during maximum voluntary contraction trials for each participant and model these trials and adjusting the strength scaling factor until the musculotendon unit activations suggest the model is fully-loaded without being overloaded. This strength scaling factor should provide a more accurate musculotendon activation scenario. 
Additionally, the model could be improved for each participant by more accurately adjusting the marker placements on the model to better represent the marker placement used on the participant. By recording the participant in a neutral posture with the full marker-set, the trial could be modeled and the marker placements in the model could be adjusted to align nearly perfectly with the markers on the participant. In this research, the markers were only adjusted when kinematic modeling failed. However, this could have led to some inaccuracy in our modeled results, especially with regard to rotation of at the upper and lower arm. Flexion/extension at the shoulder and elbow and abduction/adduction at the shoulder are driven primarily by markers on the endpoints of the segments (RSHO, RMELB, RLELB, RWRA, RWRB) which are all on easily palpable anatomical landmarks. Though, while the elbow markers are easily palpable, the skin beneath tightens up during elbow flexion, and there is significant movement of the markers during flexion/extension. This has minimal influence when driving flexion/extension and adduction/abduction, but it could have a significant influence on the rotation. While the wrist and elbow markers will assist in driving rotation of the upper and lower arms because they are in pairs, the rotation of the upper and lower arm is largely driven by the RUPA and RFRM markers, respectively. These two markers are not above easily palpable landmarks, which makes precise placement more difficult.

Aside from the limitations of the research methods and AnyBody modeling software, the outcome of the research had several limitations. First, only the right hand was used to perform the tasks. It is likely that the results would be similar with the predictive equation and heatmaps mirrored left-to-right, but lefthanded tasks were not performed so this use cannot currently be supported.

Additionally, all the lifts in this research - static and dynamic - were performed unobstructed. In a controlled environment where the workspace and task design is unconstrained, the predictive equation, heatmaps, and possibly even the origin/destination analysis would be useful. On the other hand, some jobs, such as assembly line work, require lifting to be done from a constrained posture, e.g. reaching over or around a portion of the already partially-assembled unit. Since the model was built using an unobstructed 
posture, the shoulder strain when performing a lift requiring a constrained posture would likely be higher than predicted.

Finally, the predictive equations were designed with a 15-pound load. Therefore, when the load is 15 pounds, the predicted strain values can be assumed to be an accurate estimate of the strain, and therefore the risk, on the shoulder. When the load deviates from 15-pounds, the predictive strain values should be treated as relative strains instead of actual strain.

This research provides a strong framework and proof of concept for the development of a fullfledged shoulder strain evaluation tool. Future work should perform similar analyses using much larger sample sizes of skilled laborers. However, the posture should be unconstrained, at a minimum. Ideally, consideration of other risk factors of shoulder injury should be included, the most valuable of which would be using multiple load weights. It is well-established that lifting heavy loads is a risk factor for shoulder injury (Faber et al, 2009; Ferguson et al, 2013; Garg et al, 2005; Harkness et al, 2003; Miranda et al, 2008) and strain on the shoulder will increase as the weight of the load increases. This may be possible by either a) including weight of the load as a predictor in the regression analysis or b) developing multiplier(s)/coefficient(s), either of a few discrete values based on ranges of weights or continuous nature, that can be applied to the result of the predictive equation. Participants performing the static lifting exertions throughout the reach zone with several different load weights would allow further validation of the composite index calculation and allow the load weight to be implemented and considered directly within the predictive equation.

Aside from lifting heavy loads and working at or above shoulder level, the other well-established risk factor of shoulder injury is repetitive motion (Andersen et al, 2003; Bodin et al, 2012; Frost et al, 2002; Garg et al, 2005; Leclerc et al, 2004; Miranda et al, 2008). Like implementing a factor for the weight of the load, it would be worthwhile to implement a frequency multiplier that accounts for the repetitive nature of some jobs and the influence on shoulder injury, and it could also be implemented either early in the analysis or as a modifier to the predictive equation. Since repetition and fatigue can not be 
considered in the AnyBody Modeling System, a different approach would be required. Two options to achieve this would be measuring time-to-fatigue of participants performing a repetitive task at different frequencies (repetitions per minute) or record perceived discomfort rating from participants performing a task for a set amount of time at varying frequencies.

Note, however, that a follow-up study with appropriate sample sizes, regardless of consideration of repetition or magnitude of load, could be very resource intensive. First, to comfortably perform an analysis of anyone in the working population using the resulting tool, sample sizes will need to be much larger. Since fatigue was likely becoming a factor near the end of the session, even with reasonable rest periods provided, it would be ideal to split the data collection per participant into two sessions. Which means, since the marker and EMG sensor placements would not be identical between the first and second sessions, and per the earlier recommendations, the marker placements would need to be modified between sessions and MVC exertions must be performed at both sessions. Additionally, modeling time per task can be up to 2 hours, while it is feasible to run multiple instances of the model simultaneously, the processing time is still great and requires man-power to pull the results from each task when the model completes and initiate the modeling of the next task. All things considered, this would be a vast, but worthy undertaking, especially with this research as a proof-of-concept, and can provide confidence that the resulting project would be successful and worthwhile.

Another beneficial step forward from this research would be determining a method, possibly software-driven, to predict a lifting trajectory from the origin to the destination. In this research, very similar lift trajectories were found between 4 of the 5 participants for corresponding lifts, with the $5^{\text {th }}$ participant having a very exaggerated lifting style. Since the lifting trajectories are generally very similar, this seems feasible, with the primary difficulty being the determination of the appropriate arc angle measure, i.e. steepness of the arc, to replicate the trajectories most closely. While this wouldn't allow analysis of the strain of a lifting task directly from the origin/destination, it would allow the trajectory to be approximated and the strain calculated across the lifting trajectory. 
6. CONCLUSION 
While the results of this research are not a fully-functioning lifting task analysis tool, the outcomes of this research are still valuable. A composite index, developed by Chowdhury et al. (2018), was further validated. The heatmaps and predictive equation that were developed represent a proof-of-concept and the potential for a full task analysis tool with application in workplace/task development and basic ergonomic assessment, after this analysis is performed with an appropriate sample-size. If the analysis is performed with consideration given to magnitude of load and repetition/frequency of lifting, the final tool could provide similar information to the Liberty Mutual Material Handling Tables, but with more flexibility since the location input data is continuous instead of having to stratify into intervals (10" for lifting distance, horizontal distanced of 7", 10" and 15").

The insights gained about the AnyBody Modeling System in this research are valuable to others who will use the software for similar ergonomic and human factors research. AnyBody Modeling System is powerful, but the quality of the output is defined by the quality of the model and the input. Hopefully, the AnyBody Modeling System will continue to improve and ability to integrate electromyography as input will be added. Once that occurs, AnyBody Modeling System will be a valuable research tool for understanding and better predicting the forces experienced at the joints during different activities. Without that integration, there were still some lessons learned about improving the output of the model.

While the hypothesis that the highest strain will occur at either the origin or the destination of a lifting task opposed to during the intermediate phase of the lifting task was not fully supported, the cause was an arced motion with an initial upward trajectory in many of the lifting tasks that were performed. With an understanding that some compensation for the arcing trajectory, the potential tool can reasonably be used, however a method to be predict lifting trajectory with some accuracy would be ideal.

In conclusion, this research represents a promising proof-of-concept for a full analysis tool to estimate risk of shoulder strain during unobstructed lifting tasks, and provides a viable pathway toward that goal, including the consideration of other risk factors, such as load and repetition. Ultimately, there is a strong need for a tool of this nature, and this provides a strong groundwork toward that endeavor. 


\section{REFERENCES}


Andersen JH. Risk factors in the onset of neck/shoulder pain in a prospective study of workers in industrial and service companies. Occupational and Environmental Medicine. 2003;60(9):649-54. doi:10.1136/oem.60.9.649.

Anderson N, Adams D, Bonauto D, Howard N, Silverstein B. Work-Related Musculoskeletal Disorders of the Back, Upper Extremity, and Knee in Washington State, 2002-2010; 2015.

AnyBody Modeling Systems. AnyBody Tutorial: Leson 5 (Muscle Modeling). http://www.anybodytech.com/fileadmin/AnyBody/Docs/Tutorials/chap5_Muscle_modeling/lesson5. html.

Bodin J, Ha C, Sérazin C, Descatha A, Leclerc A, Goldberg M, Roquelaure Y. Effects of Individual and Work-related Factors on Incidence of Shoulder Pain in a Large Working Population. J Occup Health. 2012;54(4):278-88. doi:10.1539/joh.11-0262-OA.

Chowdhury SK, Nimbarte AD, Hsiao H, Gopalakrishnan R, Jaridi M. A Biomechanical Shoulder Strain Index Based on Stabilizing Demand of the Shoulder Joint. Ergonomics. 2018

Crenshaw AG, Karlsson S, Gerdle B, Fridén J. Differential responses in intramuscular pressure and EMG fatigue indicators during low- vs. high-level isometric contractions to fatigue. Acta Physiologica Scandinavica. 1997;160(4):353-61. doi:10.1046/j.1365-201X.1997.00168.x.

Criswell E, Cram JR. Cram's introduction to surface electromyography. 2nd ed. Sudbury, MA: Jones and Bartlett; 2011.

Davis K, Dunning K, Jewell G, Lockey J. Cost and disability trends of work-related musculoskeletal disorders in Ohio. Occup Med (Lond). 2014;64(8):608-15. doi:10.1093/occmed/kqu126.

Engin AE, Chen S. Statistical Data Base for the Biomechanical Properties of the Human Shoulder Complex-I: Kinematics of the Shoulder Complex. J Biomech Eng. 1986;108(3):215-21. doi:10.1115/1.3138605.

Erdemir A, McLean S, Herzog W, van den Bogert, Antonie J. Model-based estimation of muscle forces exerted during movements. Clinical Biomechanics. 2007;22(2):131-54. doi:10.1016/j.clinbiomech.2006.09.005.

Faber GS, Kingma I, Kuijer, P P F M, van der Molen, H F, Hoozemans MJM, Frings-Dresen MHW, van Dieën JH. Working height, block mass and one- vs. two-handed block handling: the contribution to low back and shoulder loading during masonry work. Ergonomics. 2009;52(9):1104-18. doi:10.1080/00140130902915947.

Ferguson SA, Allread WG, Le P, Rose J, Marras WS. Shoulder Muscle Fatigue During Repetitive Tasks as Measured by Electromyography and Near-Infrared Spectroscopy. Human Factors: The Journal of the Human Factors and Ergonomics Society. 2013;55(6):1077-87. doi:10.1177/0018720813482328.

Forster E. Predicting muscle forces in the human lower limb during locomotion. VDI-Verlag; 2004. 
Frost P, Bonde JPE, Mikkelsen S, Andersen JH, Fallentin N, Kaergaard A, Thomsen JF. Risk of shoulder tendinitis in relation to shoulder loads in monotonous repetitive work. American Journal of Industrial Medicine. 2002;41(1):11-8. doi:10.1002/ajim.10019.

Garg A, Hegmann K, Kapellusch J. Short-cycle overhead work and shoulder girdle muscle fatigue. International Journal of Industrial Ergonomics. 2006;36(6):581-97. doi:10.1016/j.ergon.2006.02.002.

Green SB. How Many Subjects Does It Take To Do A Regression Analysis. Multivariate Behav Res. 1991;26(3):499-510.

Halder AM, Kuhl SG, Zobitz ME, Larson D, An KN. Effects of the Glenoid Labrum and Glenohumeral Abduction on Stability of the Shoulder Joint Through Concavity-Compression. J Bone Joint Surg Am. 2001;83(7):1062-9.

Han K, Zander T, Taylor WR, Rohlmann A. An enhanced and validated generic thoraco-lumbar spine model for prediction of muscle forces. Medical Engineering and Physics. 2012;34(6):709-16. doi:10.1016/j.medengphy.2011.09.014.

Harkness EF, Macfarlane GJ, Nahit ES, Silman AJ, McBeth J. Mechanical and psychosocial factors predict new onset shoulder pain: a prospective cohort study of newly employed workers. Occupational and Environmental Medicine. 2003;60(11):850-7.

Hignett S, McAtamney L. Rapid Entire Body Assessment (REBA). Applied Ergonomics. 2000;31(2):2015. doi:10.1016/S0003-6870(99)00039-3.

Howard N, Adams D. Work-Related Musculoskeletal Disorders of the Back, Upper Extremity, and Knee in Washington State, 2006-2015: All Washington Industries. Washington State Department of Labor \& Industries - Safety \& Health Assessment \& Research for Prevention Program. Technical Report Number 40-19-2018. Accessed on February 18, 2019 at https://www.Ini.wa.gov/Safety/Research/Wmsd/files/WMSD_TechReport\%202017ALL\%20INDUSTRIES.pdf

Hughes RE, Silverstein BA, Evanoff BA. Risk factors for work-related musculoskeletal disorders in an aluminum smelter. Am. J. Ind. Med. 1997;32(1):66-75. doi:10.1002/(SICI)1097-0274(199707)32:1

Itoi E, Kuechle DK, Newman SR, Morrey BF, An KN. Stabilising function of the biceps in stable and unstable shoulders. J Bone Joint Surg Br. 1993;75(4):546-50.

Järvholm U, Styf J, Suurkula M, Herberts P. Intramuscular pressure and muscle blood flow in supraspinatus. Europ. J. Appl. Physiol. 1988;58(3):219-24. doi:10.1007/BF00417252.

Kelly BT, Kadrmas WR, Kirkendall DT, Speer KP. Optimal normalization tests for shoulder muscle activation: An electromyographic study. Journal of Orthopaedic Research 1996. doi:10.1002/jor.1100140421. 
Körner L, Parker P, Almström C, Herberts P, Kadefors R. The relation between spectral changes of the myoelectric signal and the intramuscular pressure of human skeletal muscle. Europ. J. Appl. Physiol. 52(2):202-6. doi:10.1007/BF00433393.

Król H, Sobota G, Nawrat A. Effect of electrode position on EMG recording in pectoralis major. Journal of Human Kinetics. 2007;17:105.

Labriola JE, Lee TQ, Debski RE, McMahon PJ. Stability and instability of the glenohumeral joint: The role of shoulder muscles. Journal of Shoulder and Elbow Surgery. 2005;14(1, Supplement):S32-S38. doi:10.1016/j.jse.2004.09.014.

Leclerc A, Chastang J, Niedhammer I, Landre M, Roquelaure Y. Incidence of shoulder pain in repetitive work. Occupational and Environmental Medicine. 2004;61(1):39-44.

Lee S, An K. Dynamic glenohumeral stability provided by three heads of the deltoid muscle. Clin Orthop Relat Res. 2002(400):40-7.

Lippitt SB, Vanderhooft JE, Harris SL, Sidles JA, Harryman DT, Matsen FA. Glenohumeral stability from concavity-compression: A quantitative analysis. Journal of Shoulder and Elbow Surgery. 1993;2(1):27-35. doi:10.1016/S1058-2746(09)80134-1.

McAtamney L, Nigel Corlett E. RULA: A survey method for the investigation of work-related upper limb disorders. Applied Ergonomics. 1993;24(2):91-9. doi:10.1016/0003-6870(93)90080-S.

Miranda H, Punnett L, Viikari-Juntura E, Heliövaara M, Knekt P. Physical work and chronic shoulder disorder. Results of a prospective population-based study. Annals of the Rheumatic Diseases. 2008;67(2):218-23. doi:10.1136/ard.2007.069419.

NIOSH. Work Practices Guide for Manual Lifting. Technical Report 81-122. March, 1981.

Rasmussen J, Damsgaard M, Voigt M. Muscle recruitment by the $\min / \max$ criterion -- a comparative numerical study. Journal of Biomechanics. 2001;34(3):409-15.

Rodgers SH. A functional job analysis technique. Occup Med. 1991;7(4):679-711.

Rodosky MW, Harner CD, Fu FH. The Role of the Long Head of the Biceps Muscle and Superior Glenoid Labrum in Anterior Stability of the Shoulder. The American Journal of Sports Medicine. 1994;22(1):121-30. doi:10.1177/036354659402200119.

SENIAM. Surface Electromyography for the Non-Invasive Assessment of Muscles. www.seniam.org.

Silverstein B, Adams D. Work-related Musculoskeletal Disorders of the Neck, Back, and Upper Extremity in Washington State, 1997-2005. http://www.lni.wa.gov/safety/research/files/2007wmsdrpt.pdf.

Snook SH, Ciriello VM. The design of manual handling tasks: revised tables of maximum acceptable weights and forces. Ergonomics. 1991;34(9):1197-213. doi:10.1080/00140139108964855. 
Soslowsky LJ, Flatow EL, Bigliani LU, Pawluk RJ, Ateshian GA, Mow VC. Quantitation of in situ contact areas at the glenohumeral joint: a biomechanical study. J Orthop Res. 1992;10(4):524-34. doi:10.1002/jor.1100100407.

Stenlund B, Goldie I, Hagberg M, Hogstedt C. Shoulder tendinitis and its relation to heavy manual work and exposure to vibration. Scandinavian Journal of Work, Environment \& Health. 1993;19(1):43-9. doi:10.2307/40966108.

Terry GC, Chopp TM. Functional Anatomy of the Shoulder. J Athl Train. 2000;35(3):248-55.

U.S. Bureau of Labor Statistics. Injuries, Illnesses, and Fatalities database. 2018. Accessed 18 Apr 2019.

Winkel J, Westgaard R. Occupational and individual risk factors for shoulder-neck complaints: Part IGuidelines for the practitioner*. International Journal of Industrial Ergonomics. 1992;1(1-2):79-81. doi:10.1016/S1572-347X(00)80006-3.

$\mathrm{Xu}$ X, McGorry RW, Lin J. A regression model predicting isometric shoulder muscle activities from arm postures and shoulder joint moments. Journal of Electromyography and Kinesiology. 2014;24(3):41929. doi:10.1016/j.jelekin.2014.02.004.

Zee M de, Dalstra M, Cattaneo PM, Rasmussen J, Svensson P, Melsen B. Validation of a musculo-skeletal model of the mandible and its application to mandibular distraction osteogenesis. Journal of Biomechanics. 2007;40(6):1192-201. doi:10.1016/j.jbiomech.2006.06.024. 


\section{APPENDICES \\ Appendix A: Literature Review of Shoulder Musculoskeletal Disorder Risk Factors}

Andersen et al. (2003) performed a four year prospective study of industrial and service workers from 19 companies/plants in Denmark. Baseline assessments were performed on 3,123 workers, 1,546 of which completed three annual follow-ups. A questionnaire was sent out at baseline to assess psychosocial workplace factors (job demands, job control, and social support), intrinsic effort, and physical, emotional, and cognitive symptoms. In the same period, participants underwent a physical examination performed at the workplace to assess any pressure tenderness in the neck and shoulder, pain threshold in the lower extremities, and height, weight and BMI. Physical workplace factors were assessed by classifying tasks in the workplaces as repetitive or control tasks and aggregating tasks that had a similar level of force, repetition, neck flexion, and shoulder recovery time into 5-6 groups per site. Several workers from each group were videotaped performing the task for 10-15 minutes and the recordings were used to determine shoulder movements/second, percentage of time at more than 20 degrees neck flexion, percentage of time with no upper arm support or rest, and force requirements. At each of three annual follow-ups, participants were given a screening questionnaire to ascertain psychological workplace factors, pain status, and other symptoms. Results of the analysis showed that repetitive motions were the strongest physical risk factor for future pain. The authors noted, however, that tasks involving repetitive motion also had little recovery time and often required continuous neck flexion over $20^{\circ}$, which are also risk factors for neck/shoulder pain.

Bodin et al. (2012) recruited 83 occupational physicians between 2002 and 2005 from the Pays de la Loire region of France to assist with this research. In France, every salaried worker undergoes an annual exam be an occupational physician. The researchers assigned 15-45 half-day examination windows to each occupational physician in which they would have their patients complete a modified standardized Nordicstyle questionnaire to evaluate individual factors (age and BMI), organizational factors (time constraints, job/task rotation, high visual demand, etc.), biomechanical factors (posture, repetitive motion, perceived 
physical exertion, etc.), and psychosocial factors (support, demand, decision latitude, etc.) related to their job. A physical examination was performed to determine presence of upper-extremity MSDs at baseline. 3,710 participants were involved at baseline. A follow-up questionnaire was completed by mail or at the annual occupational physician visit between 2007 and 2009 by 1,655 eligible participants. Incidence of shoulder pain in men was associated with age, high perceived physical exertion, working with the arms above the shoulder, and working with one hand behind the trunk, however age was the most influential factor. In addition to the physical factors identified for men, high repetitiveness was also found to be a factor for women, but age was still the primary factor for incidence of shoulder pain.

Faber et al. (2009) recruited nine healthy male masons to perform a block handling task simulations in the laboratory. Blocks of four different sizes and weights $(6,11,14$ and $16 \mathrm{~kg})$ were lifted from a pallet at varying heights $(10 \mathrm{~cm}$ and $90 \mathrm{~cm})$ and placed on a wall of varying height (floor level, iliac crest level, and shoulder level), performed with one hand and with both hands. A dynamic 3D linkedsegment model was used to determine loading at the shoulder and L5/S1 region, with kinematics derived from motion capture data and ground reaction forces determined with force plates. The results of their analysis found an increase in shoulder compression as block height, pallet height, and wall height increased, supporting heavy physical load as a risk factor for shoulder MSDs. However, this research did not consider the translational forces acting on the shoulder, which are responsible for destabilization of the shoulder. Increased compressive forces, in the absence of increased translational forces, generally contribute to stability of the shoulder. Shoulder stability decreases when translational forces increase without an equivalent increase in compressive forces. Therefore, without information on the translational forces at the shoulder, the results of this research are not so straightforward.

Ferguson et al. (2013) tested the effects of varying force $(2.27 \mathrm{~kg}, 4.54 \mathrm{~kg})$, repetitions $(2,6$, and 10 repetitions per minute), and shoulder angle $\left(25^{\circ}, 60^{\circ}\right.$, and $110^{\circ}$ of forward shoulder flexion) during staticposture lifting exertions on the development of shoulder fatigue using near-infrared spectroscopy to measure tissue oxygenation and change in EMG median frequency to measure fatigue. Ten automotive 
assembly workers ( 3 female, 7 male) each attended 18 sessions over a period of 9 weeks (two days per week), with each session being dedicated to a different combination of force, repetition, and shoulder angle. The exertions were performed over a 2-hr period and pre- and post-exertion 70\% MVCs were performed. Results of the oxygenation analysis found a statistically significant effect of repetition on percent change of oxygenated hemoglobin for both the anterior deltoid and trapezius. Additionally, oxygenation in both muscles was found to increase in response to increased shoulder angle and force level. EMG analysis found an increase in median frequency in the trapezius, middle deltoid, and anterior deltoid related to increased repetitions. Increased shoulder angle and increased force were related to an increased median frequency in the trapezius and anterior deltoid, respectively.

Frost et al. (2002) performed a cross-sectional study of 4,162 workers from 19 workplaces (four food processing plants, 3 textile plants, four electronic plants, three cardboard industries, two postal sorting centers, two supermarkets, and one bank) in Demark to evaluate the effect of repetitive work and force exertion on the risk of developing shoulder tendonitis. Data was collected with a questionnaire, video recording of employees performing workplace tasks, site visits by ergonomists, and on-site physical examinations. Prevalence of shoulder tendinitis in the dominant shoulder was analyzed based on repetitive work and shoulder loads, in terms of frequency or shoulder movement, existence of micro-pauses, and force requirements using logistic regression. They found that the prevalence of shoulder tendinitis was higher among workers performing repetitive tasks than workers performing non-repetitive tasks $(\mathrm{OR}=3.12,95 \%$ $\mathrm{CI}=1.33-7.34)$. Additionally, positive associations were found between prevalence of dominant shoulder tendinitis and force requirements and lack of micro-pauses, however when limiting the analysis to only workers performing repetitive work, significance was lost between these associations. However, despite statistical insignificance, the prevalence of shoulder tendinitis was $40 \%$ higher among workers performing repetitive work with force requirements exceeding 10\% MVC.

Garg et al. (2005) recruited ten female participants to perform tasks simulating common automotive assembly tasks. A 1-minute-cycle job with four tasks was developed, and 50 repetitions of this 
task were performed by the participants for combinations of work-piece weights $(1.36 \mathrm{~kg}, 2.73 \mathrm{~kg})$, tool weights $(0.45 \mathrm{~kg}, 0.91 \mathrm{~kg}, 1.82 \mathrm{~kg})$, shoulder forward flexion $/$ elbow flexion posture $\left(60^{\circ} / 90^{\circ}, 90^{\circ} / 120^{\circ}\right.$, and $\left.120^{\circ} / 150^{\circ}\right)$, and arm-up/arm-down time $(2 \mathrm{~s}-2 \mathrm{~s}, 3 \mathrm{~s}-3 \mathrm{~s}$, and $5 \mathrm{~s}-3 \mathrm{~s})$ for a total of 54 combinations. The simulated job involved 1) holding a dumbbell with the arm to the side to simulate carrying a work-piece from the workbench to the assembly area, 2) lifting a dumbbell (work-piece weight) to the arm-up posture and maintaining the position for the appropriate time, 3) lifting a dumbbell (hand-tool weight) to the armup posture and maintaining the position for the appropriate time, and 4) resting for the appropriate time with no weight in a relaxed posture. A Borg CR-10 scale of Rating of Perceived Exertion, an 11-point fatigue scale, and an 11-point pain scale were used to subjectively quantify the physiological outcomes of the tasks, while muscle activity of the mid-deltoid and upper trapezius were recorded with surface EMG throughout the tasks. Their results showed that perceived exertion ratings were the highest, followed by fatigue ratings and pain ratings, respectively. In fact, several participants were not able to complete the tasks at the $90^{\circ} / 120^{\circ}$ and $120^{\circ} / 150^{\circ}$ postures, specifically with heavier hand-tool weight, work-piece weight, and the $2 \mathrm{~s}-2 \mathrm{~s}$ arm-up/arm-down timing. Work-piece weight, hand-tool weight, arm-up/arm-down timing, and posture were all found to significantly affect rating of perceived exertion, pain, and discomfort, however when looking at the median power frequency of the surface EMG, an indicator of fatigue, no consistent patterns were found and some less demanding tasks were more fatiguing than some of the higher demand tasks.

Harkness et al. (2003) performed a two year prospective study of new employees across twelve diverse workplaces. A total of 1081 participants were surveyed on their work-related risk factors and shoulder pain status. Of those surveyed, 803 participants were free from shoulder pain at onset. Follow-ups at 12 and 24 months were completed by 638 and 476 of the participants, respectively. New onset shoulder pain was reported by 93 participants at 12 months and 73 participants at 24 months. Lifting heavy weights with one or two hands, carrying on shoulder, lifting at or above shoulder, pushing or pulling, and working with hands above shoulder level were associated with an increased risk of shoulder pain at follow-up. 
Hughes et al. (1997) recruited 107 male employees from an aluminum smelter from three job categories (carbon setters, crane operators, and carbon plant workers) to 1) undergo a physical examination that includes a battery of tests to determine musculoskeletal issues with the neck, back, shoulder, elbow, and wrist and 2) complete a self-administered questionnaire to determine the psychosocial factors of the work they perform. Additionally, a job analysis was performed using a modified job-surveillance checklist method and years in the position to determine years of exposure of each worker for each of 37 potential risk factors (Arm/hand above shoulder, asymmetric lift, climbing stairs/ladder, full elbow flexion, pinch grip, walking, twisting, etc.). Univariate analysis of the factors was performed with outcome variables of "Work-related musculoskeletal disorder on interview" and "Work-related musculoskeletal disorder on interview and exam", revealing several factors with a significant association with exposure, including working with the arm/hand above the shoulder, elbow use at greater than mid-torso, gripping slippery objects, lifting/carrying, pushing/pulling, twisting/rotating the forearm, and holding more than $2.7 \mathrm{~kg}$ per hand. Multiple logistic regression was also performed to determine the primary predictors of shoulder workrelated musculoskeletal disorders. The primary predictors of shoulder work-related musculoskeletal disorders on interview alone were age, smoking, low decision latitude, high job demand, and years of forearm twisting. When performing the analysis of shoulder work-related musculoskeletal disorders on interview and exam, the primary factors are age, health, low decision latitude, and years of forearm twisting. Finally, $40 \%$ of the workers reported recurring shoulder problems with $24 \%$ reporting symptoms within the prior week.

Leclerc et al. (2004) conducted a prospective cohort study on 598 workers in five sectors requiring repetitive work to assess occupational factors for the onset of shoulder pain. Shoulder complaints and physical workload information were obtained with a self-reported questionnaire. For women, the strongest predictors of incidence of shoulder pain were use of vibrating tools and working with the hands above shoulder level, while repetitive use of a tool was the strongest predictor for men. 
Miranda et al. (2008) conducted a national survey among a representative sample of 909 Finnish adults. A total of 883 participants remained after excluding those with diagnosed shoulder disorders at baseline. Chronic shoulder disorders were diagnosed in 63 participants using a standardized protocol. Exposure to repetitive motions and vibration at baseline increased the risk of shoulder disorders, with adjusted ORs of 2.3 (95\% CI: $1.3-4.1)$ and 2.5 (95\% CI: 1.2-5.2). Combinations of exposures increased the risk further, with exposure to at least 3 factors resulting in adjust OR of almost 4. Statistically significant risk factors for men were vibration and repetitive movements, while the statistically significant risk factors for women were lifting heavy loads and working in awkward postures.

Stenlund et al. (1993) performed structured interviews and physical examinations of three categories of construction industry workers [bricklayers $(n=54)$, rockblasters $(n=55)$, and foremen $(n=98)$ ] with the goal of determining work-related risk factors of shoulder tendonitis and muscle attachment inflammation. Rockblasters were found to be at the highest risk of shoulder tendinitis, which the researchers attributed to the vibration from jackhammers and other handheld tools and heavy static loads, such as jackhammers and large rocks. Bricklayers, however, were found to have the lowest risk of shoulder tendonitis and inflammation. Overall, vibration was found to be a significant risk factor, while years of manual work and cumulative lifted load were not found to be significant factors for the development of shoulder tendinitis or inflammation in bricklayers and rockblasters. 


\section{Appendix B: Specific Aim 1 Consent Form}

\section{Only Minimal Risk \\ Consent Information Form (without HIPAA)}

Principal Investigator: Dr. Ashish Nimbarte

Department: $\quad$ Industrial and Management Systems Engineering

Protocol Number: $\quad$ \#1603052164

Study Title: Determination of the optimal muscle recruitment equation for shoulder modeling using AnyBody Modeling System

Co-Investigator(s) Christopher W. Moore

Sponsor (if any) N/A

\section{Contact Persons}

In the event you experience any side effects or injury related to this research, you should contact Dr. Ashish Nimbarte at (304) 293-9473. (After hours contact: Christopher Moore at (304) 685-5532). If you have any questions, concerns, or complaints about this research, you can contact Christopher Moore at (304) 685-5532.

For information regarding your rights as a research subject, to discuss problems, concerns, or suggestions related to the research, to obtain information or offer input about the research, contact the Office of Research Integrity and Compliance at (304) 293-7073.

In addition if you would like to discuss problems, concerns, have suggestions related to research, or would like to offer input about the research, contact the Office of Research Integrity and Compliance at 304-293-7073.

\section{Introduction}

You, have been asked to participate in this research study, which has been explained to you by Christopher Moore. This study is being conducted by Dr. Ashish Nimbarte and Christophe Moore in the Department of Industrial and Management Systems Engineering at West Virginia University.

\section{Purpose(s) of the Study}

Advances In technology have provided us with the computing power and ability to do in-depth analyses and simulations of the human body using musculoskeletal modeling software. However, the software relies on math equations to determine how activity is divided up to the muscles to perform a given task. The purpose of this study is to determine which equation within the AnyBody Modeling System software provides the most accurate representation of the actual activities of the muscles with a human subject performing the task by comparing the muscle activity predicted by the

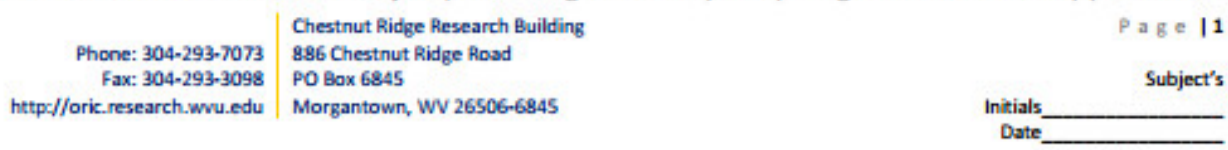




\section{We-WestViriginiaUniversity.}

Human Research Protocol

Only Minimal Risk Consent Form

Without HIPAA

model with the actual muscle activity data of the subject.

This research study is the first of three parts of a dissertation project toward a Doctorate in Occupational Safety and Health.

\section{Description of Procedures}

This study uses small sensors, attached to the skin with tape, to measure the muscle activity of several muscles. Small reflective markers taped on the upper body will be tracked with special cameras to measure the posture. Participants will exert force against a padded, non-moving surface followed by three straight-arm lifting tasks involving lifting a five pound weight to shoulder level while keeping the arm straight. The study will take about an hour for you to complete. Before signing the consent form, you will be introduced to the study procedures and equipment.

\section{Discomforts}

Delayed onset muscle soreness and acute muscle discomfort are possible during the maximum exertions. Acute muscle discomfort should subside at the completion of the task. Delayed onset muscle soreness should subside within 1-2 days. Electromyography (muscle activity) sensors and motion tracking markers are affixed to the participant's skin with double-sided tape. In rare cases, allergic reaction to the tape could occur, so potential participants with adhesive allergies are recommended not to join the study.

\section{Alternatives}

Your participation in this study is voluntary and you may choose not to participate.

\section{Benefits}

You may not receive any direct benefit from this study. The knowledge gained from this study may eventually benefit others.

\section{Financial Considerations}

There are no special fees for participating in this study and no compensation will be provided.

\section{Confidentiality}

Any information about you that is obtained as a result of your participation in this research will be kept as confidential as legally possible. Your research records and test results, just like hospital records, may be subpoenaed by court order or may be inspected by the study sponsor or federal regulatory authorities (including the FDA if applicable) without your additional consent.

In addition, there are certain instances where the researcher is legally required to give information to the appropriate authorities. These would include mandatory reporting of infectious diseases, mandatory reporting of information about behavior that is imminently dangerous to your child or to others, such as suicide, child abuse, etc.

\begin{tabular}{|c|c|c|c|}
\hline $\begin{array}{r}\text { Phone: } 304-293-7073 \\
\text { Fax: } 304-293-3098 \\
\text { hetp//foric.research.wwu.edu }\end{array}$ & $\begin{array}{l}\text { Chestnut Ridge Research Building } \\
886 \text { Chestnut Ridge Rosd } \\
\text { PO Bax } 6845 \\
\text { Morgantown, WV 26506-6845 }\end{array}$ & Intitials_ & $\begin{array}{l}P \text { a } g \text { e } 12 \\
\text { Subject's }\end{array}$ \\
\hline & & Date & \\
\hline
\end{tabular}




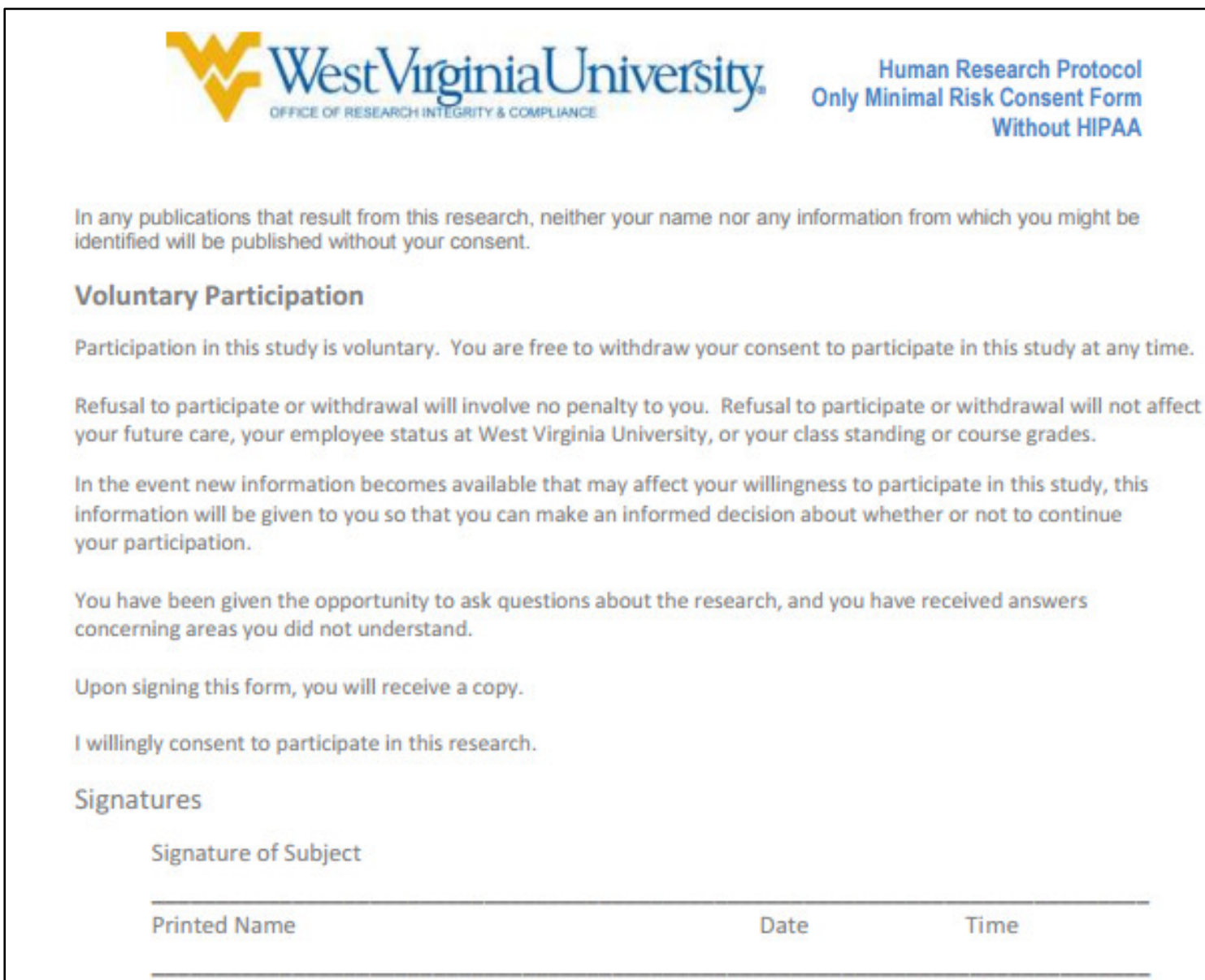

The participant has had the opportunity to have questions addressed. The participant willingly agrees to be in the study.

Signature of Investigator or Co-Investigator

\begin{tabular}{lll}
\hline Printed Name & Date & Time \\
\hline
\end{tabular}

\begin{tabular}{|c|c|c|c|}
\hline Phone: $304-293-7073$ & $\begin{array}{l}\text { Chestnut Ridge Research Building } \\
896 \text { Chestnut Ridge Rosd }\end{array}$ & & $P$ a $g \in \mid 3$ \\
\hline Fax: 304-293-3098 & PO Box 6845 & & Subject's \\
\hline http-//oric.research.wwu.edu & Morgantown, WV 26506-6845 & Initials_. & \\
\hline
\end{tabular}

Approved:9-Dec-2016Expires:8-Dec-2017Number:1603052164 


\section{Appendix C: Physical Activity Readiness Questionnaire}

\section{PAR-Q Form}

Name:

Date:

DOB:

Height: Weight:

Health Care Provider: Phone:

\section{Questions}

Has your health care provider ever said that you have a heart condition and that you should only perform physical activity recommended by a doctor?

Do you feel pain in your chest when performing physical activity?

Have you experienced chest pain when NOT performing physical activity in the last month?

Do you lose your balance because of dizziness or have you lost consciousness recently?

Do you have any bone or joint problems (back, knee, hip, etc.) such as arthritis, which could be aggravated through physical activity?

$\square$ Yes $\square$ No

$\square$ Yes $\square$ No

$\square$ Yes $\square$ No

$\square$ Yes $\square$ No

$\square$ Yes $\square$ No

Is your doctor currently prescribing you medications for high blood pressure $\square$ Yes $\quad \square$ No or a heart condition?

Is there any reason why you should not participate in physical activity? Reason:

Do you currently exercise on a regular basis $(3+$ times per week)?

$\square$ Yes No

If Yes to Any Questions:

If No to All Questions: 
Appendix D: Maximum Voluntary Contraction Guidesheets

\section{MVC \#1}

Muscles activated: Deltoid, Pectoralis Major, Supraspinatus

With the shoulder abducted to $90^{\circ}$ in the scapular plane and $45^{\circ}$ internally rotated and elbow extended, the participant will abduct the arm with maximum force with resistant force applied at the wrist.

\section{(Kelly et al., 1996)}
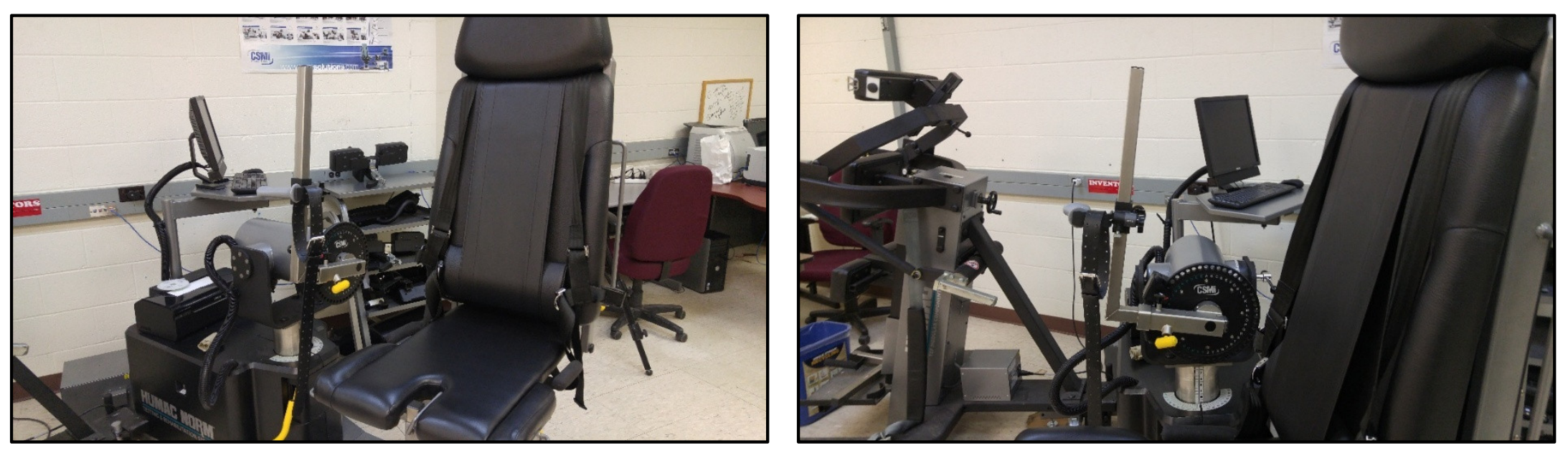

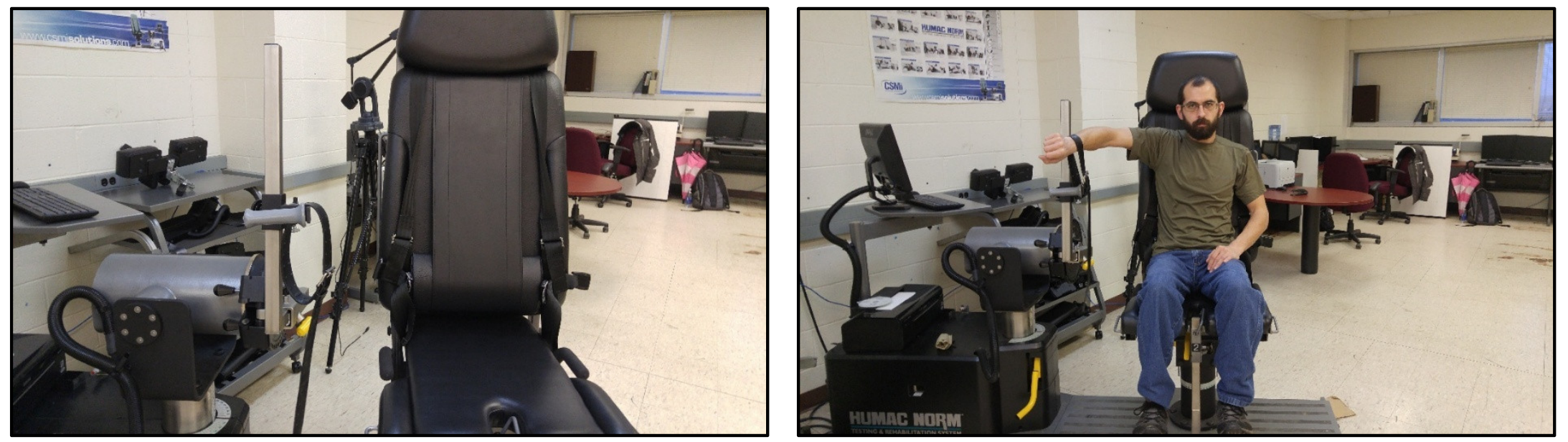

\section{MVC \#2}

Muscles activated: Anterior and Middle Deltoid, Infraspinatus, Supraspinatus

With the shoulder flexed to $125^{\circ}$, the participant will resist a force applied above the elbow toward the inferior angle of the scapula to de-rotate the scapula.

(Xu et al., 2014)
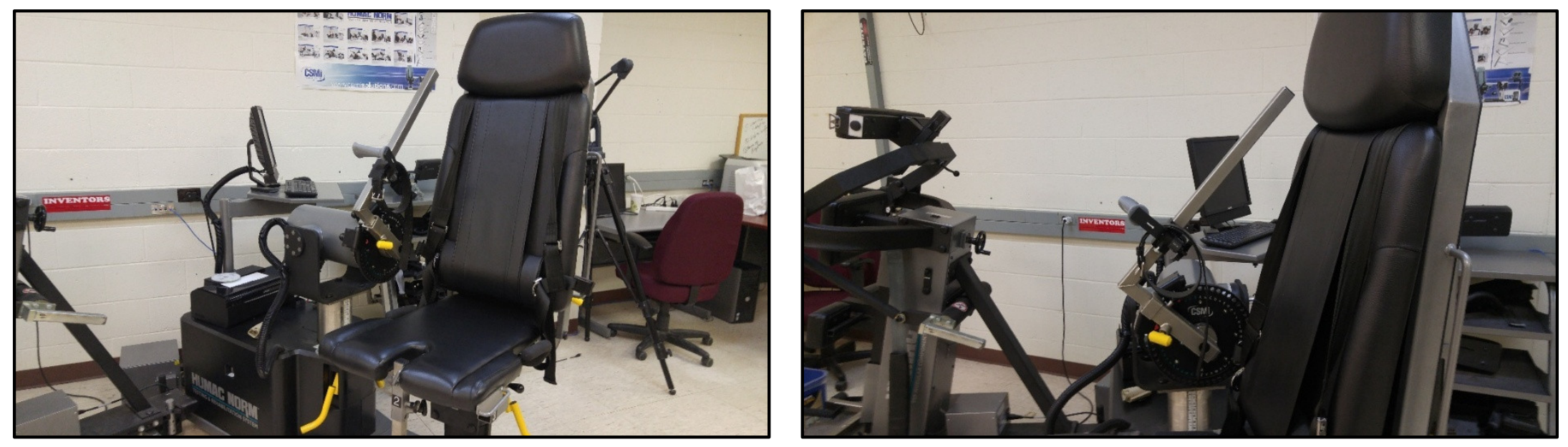

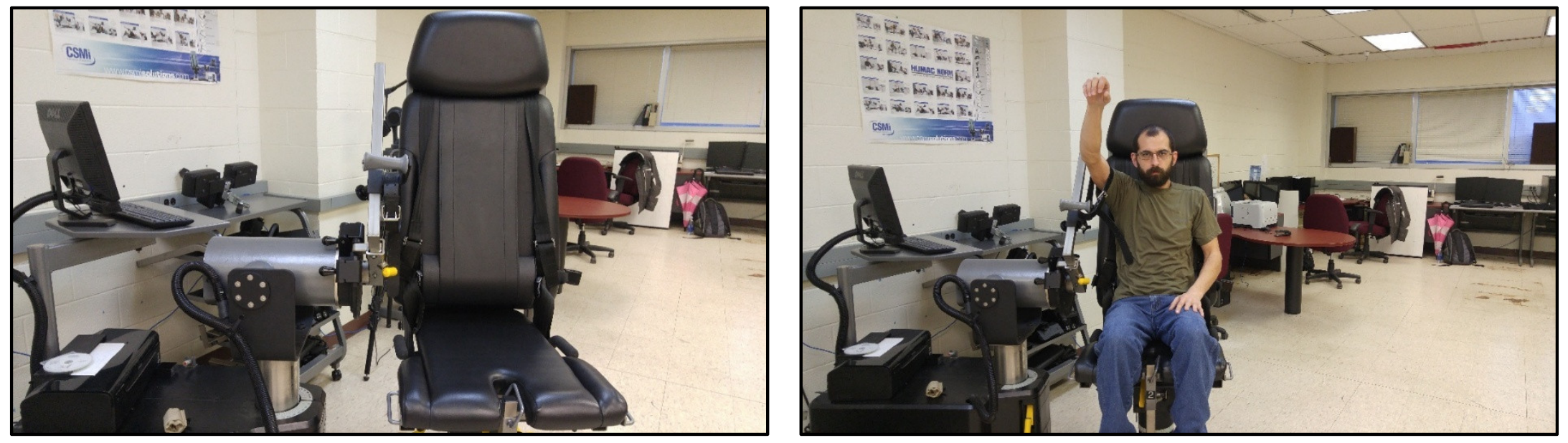

\section{MVC \#3}

\section{Muscles activated: Latissimus Dorsi}

With the shoulder abducted to $90^{\circ}$ in the scapular plane and $90^{\circ}$ elbow flexion, the participant will internally rotate with maximal force with resistive force applied at the wrist.

(Xu et al., 2014) 

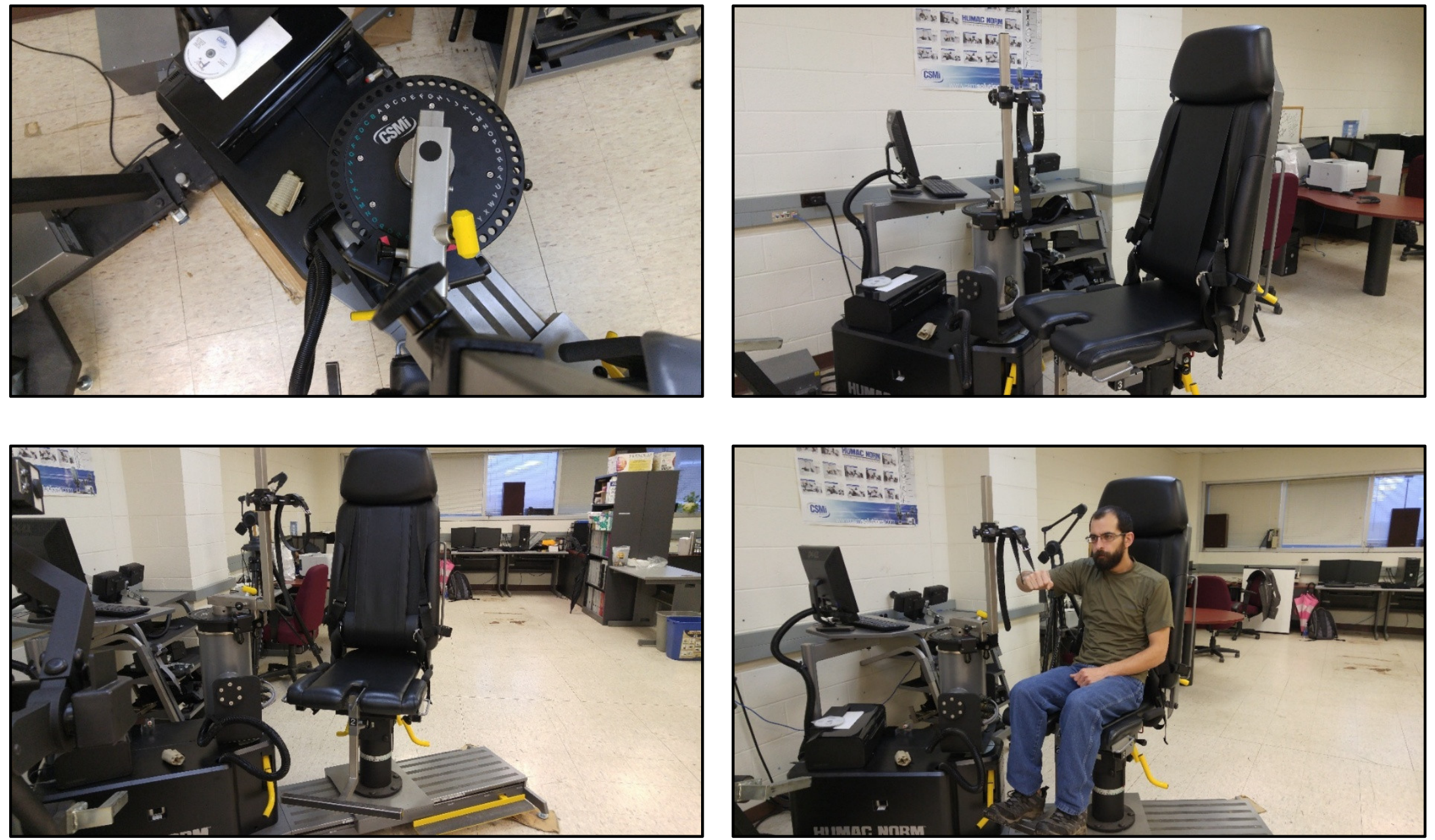

\section{MVC \#4}

Muscles activated: Biceps

With the elbow flexed to $90^{\circ}$, the participant will flex the arm with maximum force against a resistive force at the wrist.

(Xu et al., 2014) 

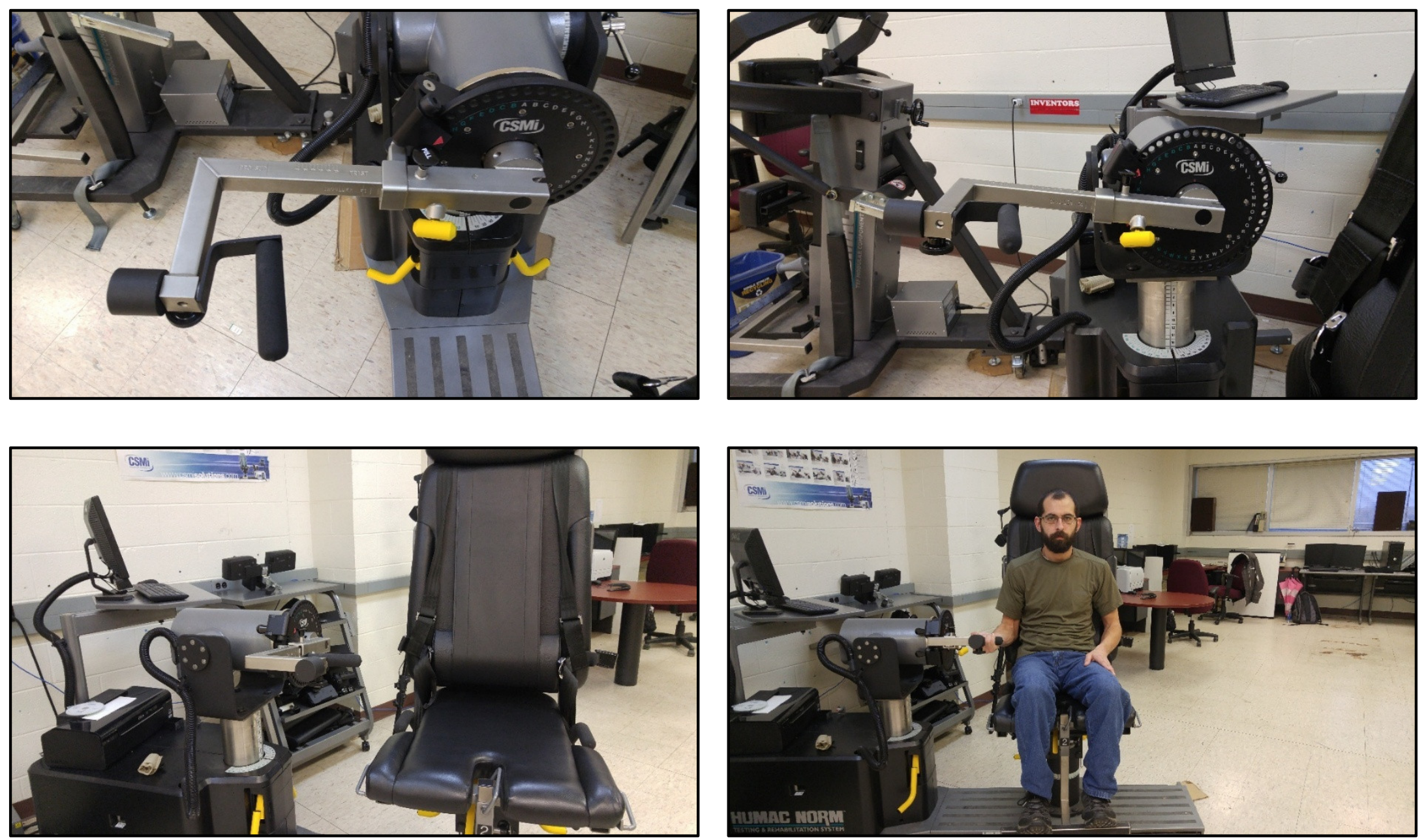

\section{MVC \#5}

\section{Muscles activated: Triceps}

With the elbow flexed to $90^{\circ}$, the participant will extend their elbow with maximum force against a resistive force applied at the wrist. 

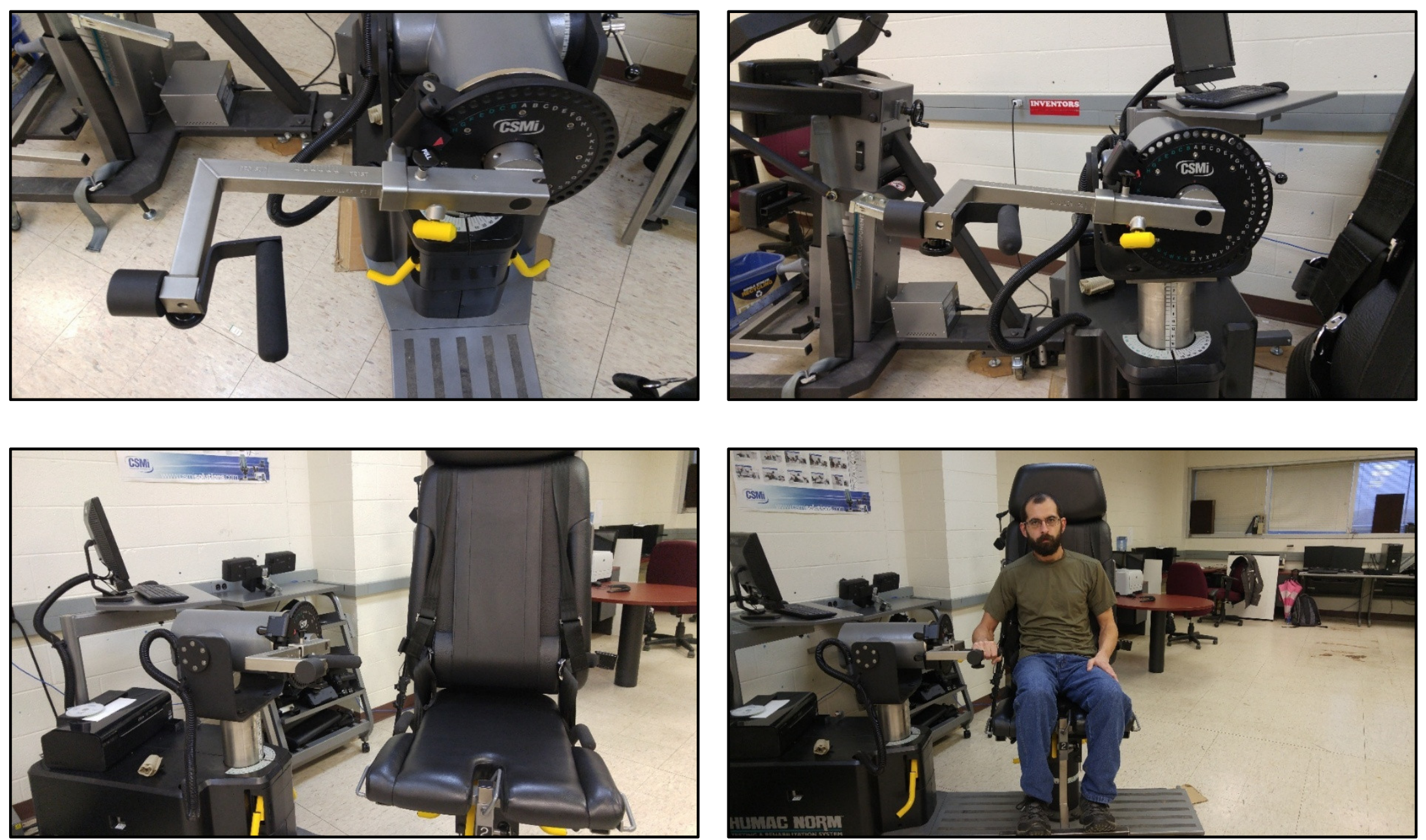

\section{MVC \#6}

Muscles activated: Pectoralis Major 
With neutral posture of the shoulder and $90^{\circ}$ of elbow flexion, the participant will internally rotate with maximal force with resistive force applied at the wrist.

\section{(Kelly et al., 1996)}
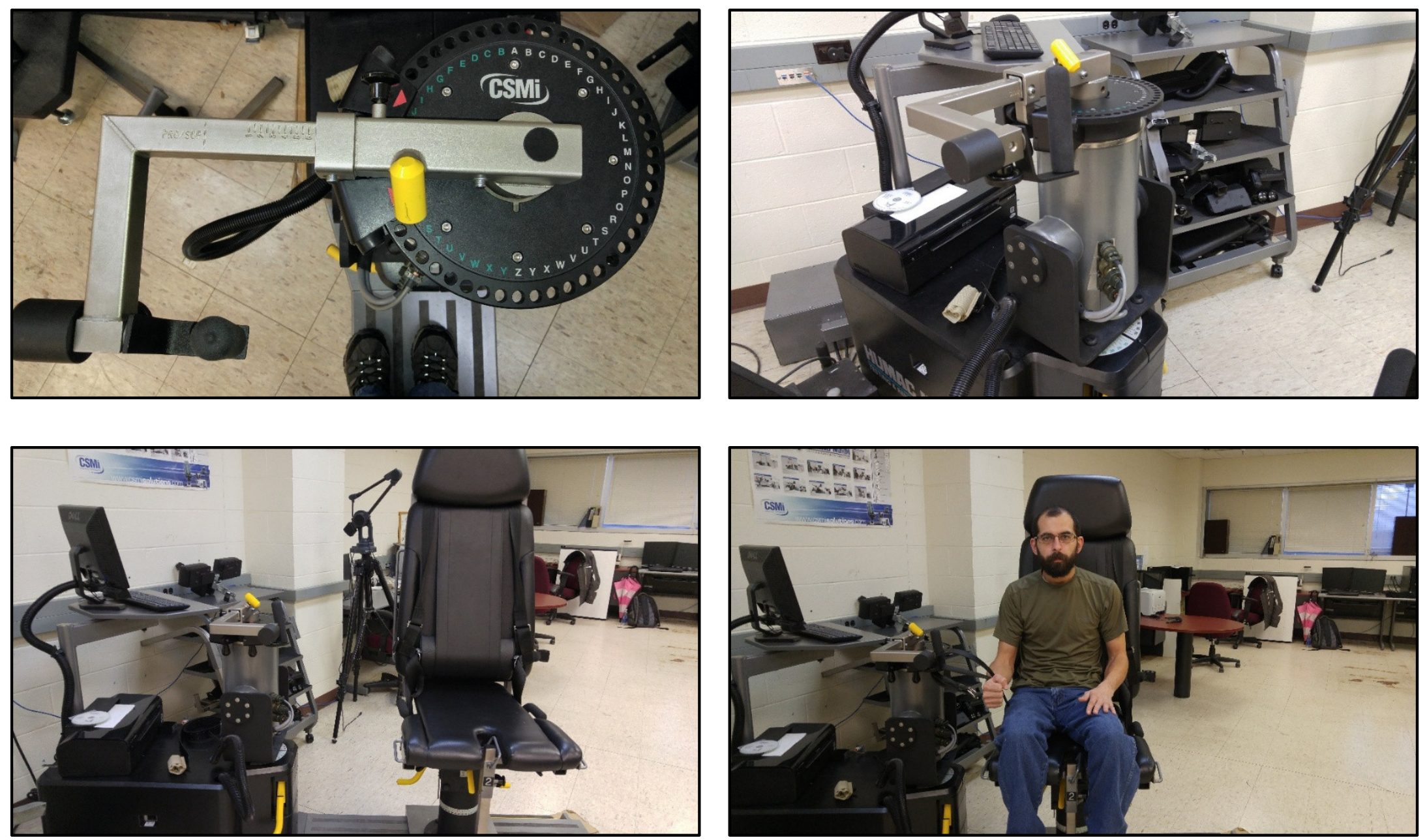

MVC \#7 
With the shoulder abducted to $45^{\circ}$ and $90^{\circ}$ of elbow flexion, the participant will internally rotate their arm with a resistive force applied at the wrist.

(Xu et al., 2014)
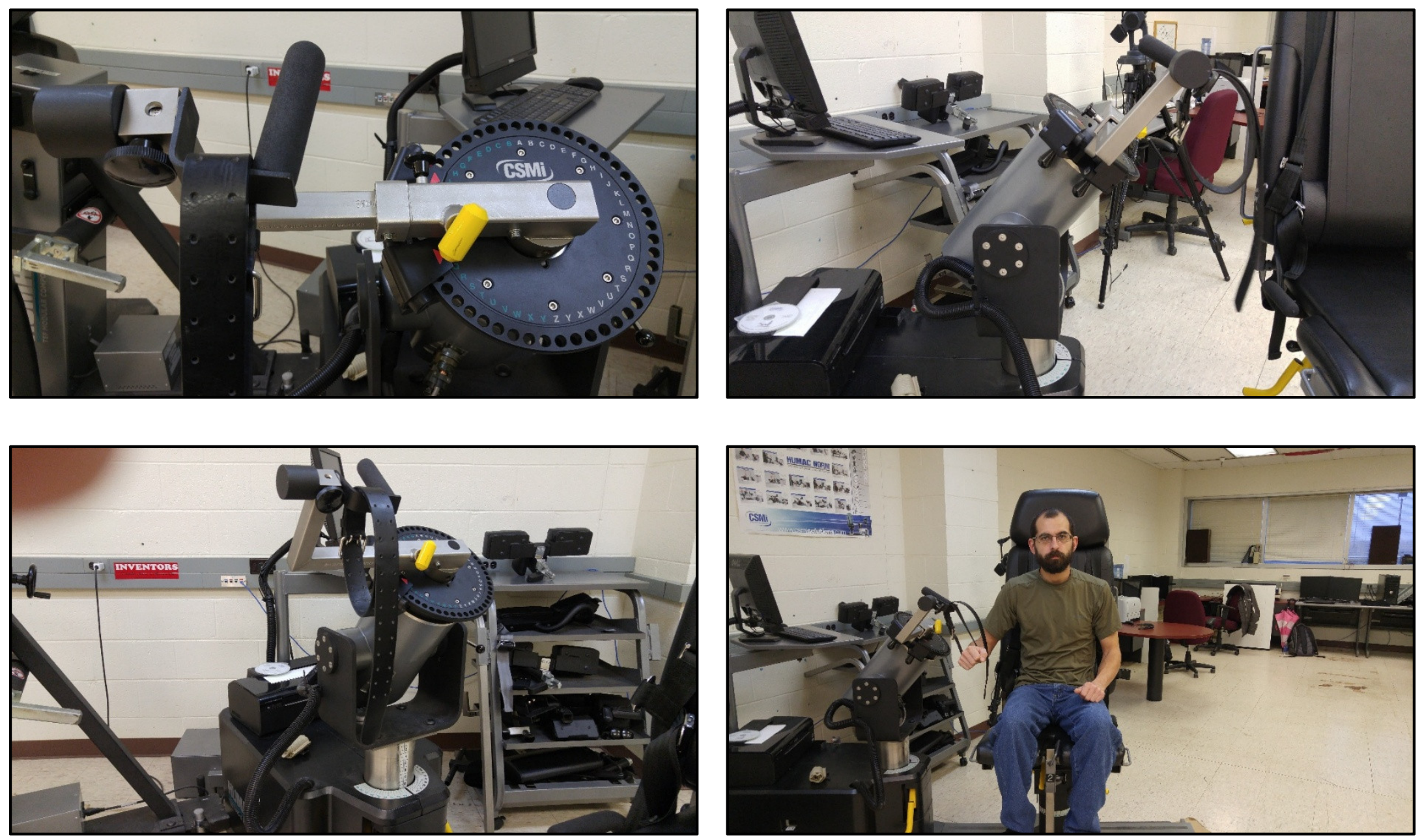


\section{Appendix E: MATLAB Codes for Specific Aim 1 Data Processing}

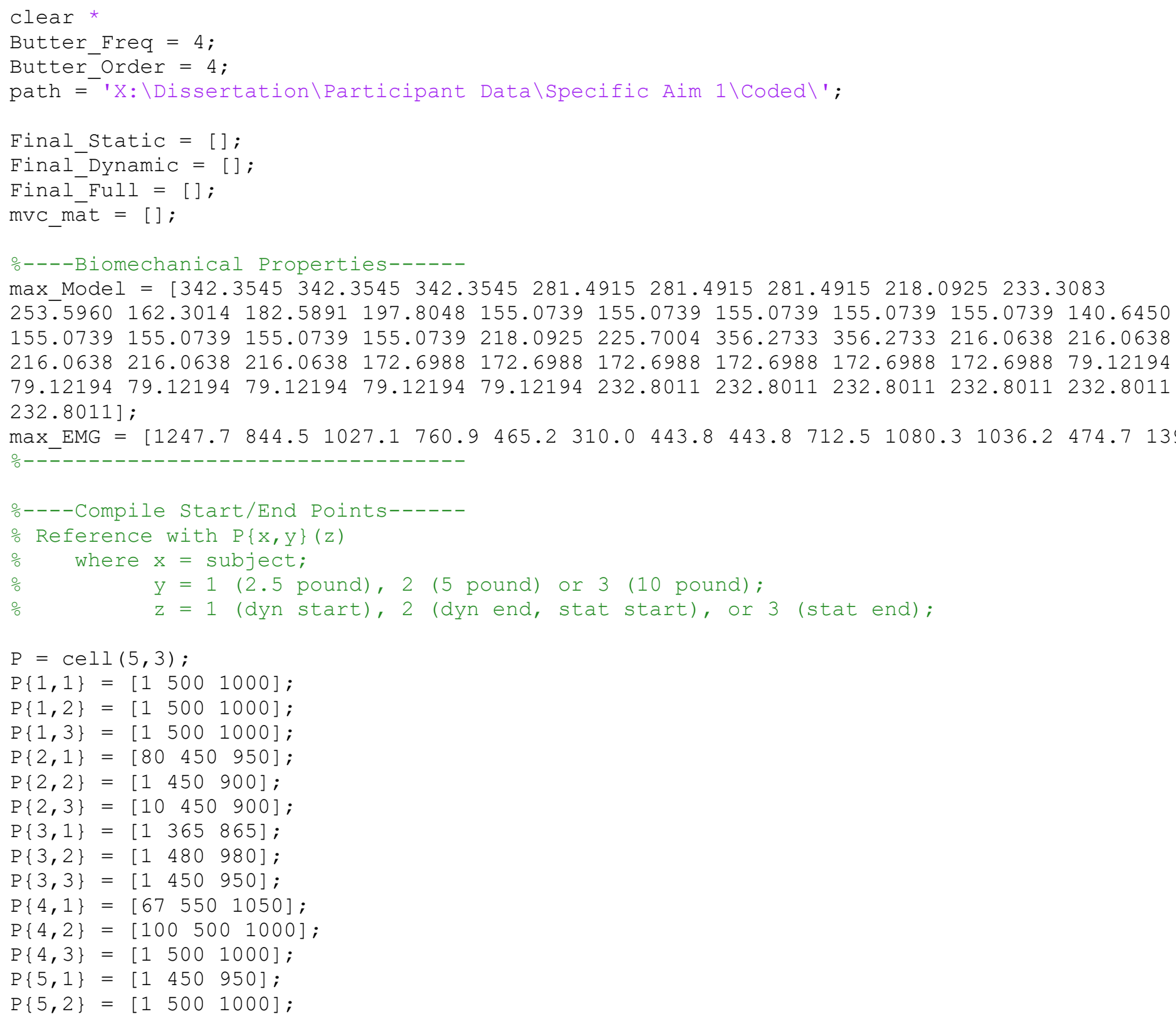


for $i=1: 5$

i

cd (strcat (path, 'S', num2str(i), '\MVC\'));

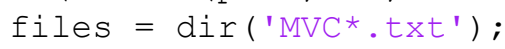

for $j=1$ :length (files)

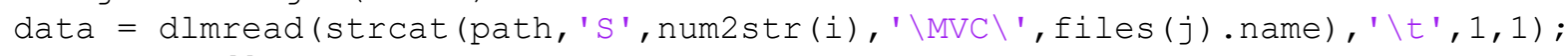

tmp_mat $=[]$;

for $\mathrm{k}=1: 13$

tmp_mat $(:, \mathrm{k})=$ resample $\left(\mathrm{EMG}_{\text {_Filt }}\right.$ (Butter_Order,Butter_Freq, data $\left.\left.(:, \mathrm{k})\right), 1,10\right)$;

end

$[r, \sim]=\operatorname{size}($ mvc_mat);

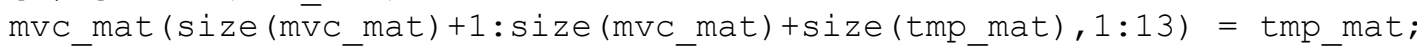

cleàr tmp_mat;

end

mvc_final (i, 1:13) = max (mvc mat);

mvc_mat $=[]$;

cd (strcat (path, 'S', num2str(i), '\EMG\')) ;

files $=\operatorname{dir}\left({ }^{*} \cdot\right.$.txt');

for $j=1$ :length (files)

$[\sim$, coded_wt, $\sim=$ fileparts (files $(j)$. name) $;$

num_wt $=$ str2num (strrep (strrep (strrep (coded_wt, '25P', '1'), '50P', '2'), '100P', '3') );

pt_x $=P\{i$, num_wt $\}(1)$;

pt_y $=P\{i$, num_wt $\}(2)$;

pt_z $=P\{i$, num_wt $\}(3)$;

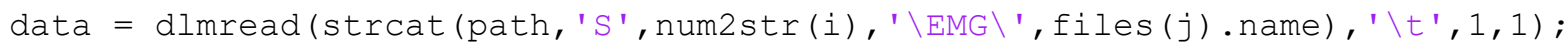

mat_emg $=[]$;

for ${ }^{-} \mathrm{k}=1: 13$

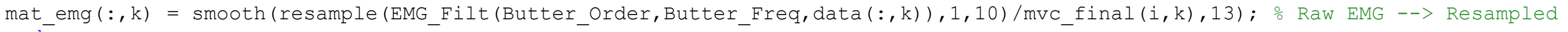
end

tmp_mat $=$ mat_emg $($ pt_y:pt_z, : $) .{ }^{\star} \max E M G$;

emg_stat_prop $\overline{-}^{-}$mean $($tmp_mat $) / \operatorname{sum}(\operatorname{me} \bar{e}$ (tmp_mat) $)$;

cd (strcat (path, 'S', num2str(i), '\MODEL\', coded_wt))

rs_files $=\operatorname{dir}\left({ }^{*}\right.$. txt') ;

for $\mathrm{k}=1$ :length (rs files)

$[\sim$, coded_rs, $\sim=$ fileparts (rs_files $(\mathrm{k})$.name);

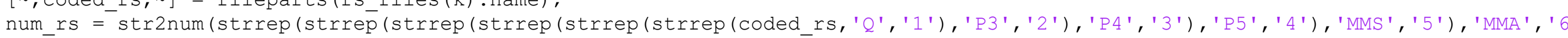

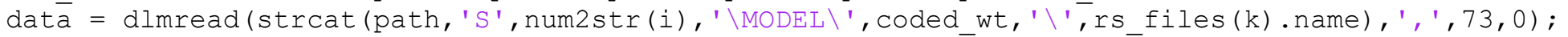

clear mat_model; 
mat_model $(:, 1)=\operatorname{sum}\left(\operatorname{data}(:, 7: 12) .{ }^{\star} \max \operatorname{Model}(7: 12), 2\right) / \max$ EMG $(1)$;

mat_model $(:, 2)=\operatorname{sum}\left(\operatorname{data}(:, 4: 6) .{ }^{\star} \max \bar{M}_{-}\right.$del $\left.(4: 6), 2\right) / \max E \overline{\mathrm{M}} G(2)$;

mat model $(:, 3)=\operatorname{sum}\left(\right.$ data $\left.(:, 1: 3) .{ }^{\star} \max \operatorname{Model}(1: 3), 2\right) / \max E M G(3)$;

mat_model $(:, 4)=\operatorname{sum}\left(\operatorname{data}(:, 18: 22) .{ }^{*} \max { }_{-} \operatorname{Model}(18: 22), 2\right) \overline{\mathrm{max}} \mathrm{EMG}(4)$;

mat model $(:, 5)=\operatorname{sum}\left(\operatorname{data}(:, 15: 17) .{ }^{\star} \max \operatorname{Model}(15: 17), 2\right) / \max { }_{\text {EMG }}(5)$;

mat_model $(:, 6)=\operatorname{sum}\left(\right.$ data $\left.(:, 13: 14) .{ }^{\star} \max \_\operatorname{Model}(13: 14), 2\right) / \max E M G(6)$;

mat_model $(:, 7)=\operatorname{sum}\left(\operatorname{data}(:, 23: 24) .{ }^{*} \max { }_{-} \operatorname{Model}(23: 24), 2\right) / \max { }_{\text {EMG }}(7)$;

mat model $(:, 8)=\operatorname{sum}\left(\operatorname{data}(:, 23: 24) .{ }^{\star} \max \operatorname{Model}(23: 24), 2\right) / \max \operatorname{EMG}(8)$;

mat_model $(:, 9)=\operatorname{sum}\left(\operatorname{data}(:, 25: 26) .{ }^{*} \max { }_{-} \operatorname{Model}(25: 26), 2\right) / \max { }_{-} \mathrm{EMG}(9)$;

mat model $(:, 10)=\operatorname{sum}\left(\operatorname{data}(:, 27: 31) \cdot{ }^{\star} \max \operatorname{Model}(27: 31), 2\right) / \max \bar{x} \operatorname{EMG}(10)$;

mat_model $(:, 11)=\operatorname{sum}\left(\operatorname{data}(:, 32: 37) .{ }^{\star} \max \_\operatorname{Model}(32: 37), 2\right) / \max$ EMG $(11)$;

mat_model $(:, 12)=\operatorname{sum}\left(\operatorname{data}(:, 38: 43) .{ }^{\star} \max \operatorname{Model}(38: 43), 2\right) / \max E M G(12)$;

mat_model $(:, 13)=\operatorname{sum}\left(\operatorname{data}(:, 44: 49) .{ }^{\star} \max { }_{-} \operatorname{Model}(44: 49), 2\right) / \max { }_{-} \mathrm{EMG}(13)$;

\% Full/Dynamic analysis and static preparation

[len, ] = size (mat_emg);

for $m=1: 13$

o Full Analysis

[corr_rho_f, corr_pval_f] $=\operatorname{corr}\left(\operatorname{mat} \_m o d e l(1: 1 e n, m), \operatorname{mat} e m g(:, m)\right)$;

rmse_f $=\overline{\operatorname{sqrt}}\left(\operatorname{sum}\left((\operatorname{mat} \operatorname{model}(1: \operatorname{len}, \overline{\mathrm{m}})-\operatorname{mat} \operatorname{emg}(:, \mathrm{m})) \cdot{ }^{\wedge} 2 \overline{)} / \mathrm{len}\right)\right.$;

- Dynamic Analysis

[corr rho d, corr pval d] = corr (mat model (pt $x: p t y, m)$, mat emg (pt $x: p t y, m))$;

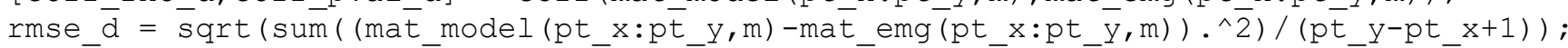

$[r, \sim]=\operatorname{size}($ Final_Dynamic);

- Add data to the Dynamic/Full Analysis Matrix

․ Format: [Subj] [Weight] [Musc] [RecruitStrat] [Corr] [Pval] [RMSE]

Final_Dynamic $(r+1,:)=$ [i num_wt m num_rs corr_rho_d corr_pval_d rmse_d];

Final Full $(r+1,:)=[i$ num wt m num rs corr rhōf corr pval f rmse f];

end

o Static Analysis Preparation

tmp_mat $=$ mat_model (pt_y:pt_z, :).*max_EMG;

model stat prop $=$ mean (tmp mat) / sum (méan (tmp mat));

[corr_rho_s, corr_pval_s] = corr(model_stat_prop', emg_stat_prop', 'type', 'Spearman') ;

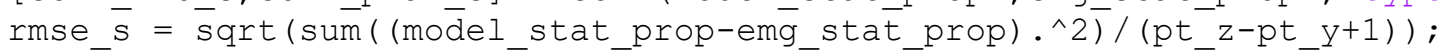

$[r, \sim]=\operatorname{size}($ Final Static) $;$

\% Add data to the Static Analysis Matrix

o Format: [Subj] [Weight] [RecruitStrat] [SpearCorr] [Pval] [RMSE]

Final_static $(r+1,:)=[i$ num_wt num_rs corr_rho_s corr_pval_s rmse_s];

end

end

Final_Static $=$ real(Final_Static); 
Final_Dynamic = real (Final_Dynamic); Final_Full = real (Final_Fūll);

end

function $[y]=$ EMG_Filt (ord, freq, $x$ ) beg $=1$

fin $=60$;

$\mathrm{s}=\mathrm{floor}(\operatorname{size}(\mathrm{x}) / 1000)$;

$r=s * 1000$;

$y=f f t(x(1: r))$;

$\mathrm{Y}\left((\mathrm{beg}-1){ }^{*} \mathrm{~S}+1: \mathrm{fin}{ }^{\mathrm{S}},:\right)=0$

$y(r-($ beg -1$) * s: r-f i n * s-1,:)=0$;

$\mathrm{y}=$ ifft (y, 'symmetric');

$[\mathrm{b}, \mathrm{a}]=$ butter (ord, freq/500);

end

$y=$ filtfilt (b, a, abs (y-mean (y))); 


\title{
Appendix F: Specific Aim 2 Consent Form
}

\section{WestViriniaianiversity,}

\author{
Human Research Protocol \\ Only Minimal Risk Consent Form \\ Without HIPAA
}

\section{Only Minimal Risk \\ Consent Information Form (without HIPAA)}

Principal Investigator

Dr. Ashish Nimbarte

Department

Industrial and Management Systems Engineering

Protocol Number

$\# 1608234050$

Study Title

Development of Predictive Equations to Estimate Shoulder Strain Based on

Location of a Load within the Working Space.

Co-Investigator(s)

Christopher W. Moore

Sponsor

N/A

\section{Contact Persons}

In the event you experience any side effects or injury related to this research, you should contact Dr. Ashish Nimbarte at (304) 293-9473 or Christopher Moore at (304) 685-5532 (After hours contact: Dr. Ashish Nimbarte at (225) 226-8813). If you have any questions, concerns, or complaints about this research, you can contact Christopher Moore at (304) 6855532.

For information regarding your rights as a research subject, to discuss problems, concerns, or suggestions related to the research, to obtain information or offer input about the research, contact the Office of Research Integrity and Compliance at (304) 293-7073.

In addition if you would like to discuss problems, concerns, have suggestions related to research, or would like to offer input about the research, contact the Office of Research Integrity and Compliance at 304-293-7073.

\section{Introduction}

You, have been asked to participate in this research study, which has been explained to you by Christopher Moore. This study is being conducted by Dr. Ashish Nimbarte and Christopher Moore in the Department of Industrial and Management Systems Engineering at West Virginia University.

\section{Purpose(s) of the Study}

The purpose of this study is to develop equations that can be used to predict the risk of shoulder injury when lifting an object based on where it is with relation to your body. Ultimately, this information will be used to create a tool that can be used by industry safety professionals to determine risk of shoulder injury of a task that involves lifting and moving a weight.

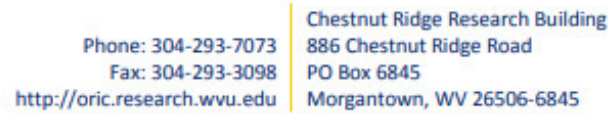

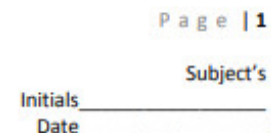




\section{Description of Procedures}

Consenting participants will first be fitted with electromyography sensors and motion capture markers.

Electromyography sensors, used to detect the activity of the underlying muscles, will be attached to the skin above several shoulder muscles with double-sided tape. Motion capture markers, which can be tracked by special cameras that emit and detect infrared light that reflects off of the markers, are attached with double-sided tape to specific anatomical landmarks of the upper body.

Maximum voluntary contractions are designed to work one or more muscles as hard as possible to find its maximum activity. Each of these 7 tasks will be done by assuming a specific arm posture and exerting as much force as possible in the manner directed against a padded, non-moving surface or strap.

The lifting tasks involve lifting a 10 pound weight suspended by a rope. For each of five different heights, you will move to different places on a grid on the floor with 8 " intervals, stand upright facing forward in a natural position, grasp the suspended weight and lift it enough to support its entire weight. You will hold the weight for a period of 5-10 seconds and slowly release the weight. If you cannot reach the weight without bending or rotating your torso, the location will be skipped for that height. Total number of lifting tasks will depend on which locations are within reach. Rest periods will be given between lifts.

This research will take approximately 4 hours to complete.

\section{Discomforts}

Muscle fatigue is possible during the data collection, but should subside shortly after completion. Delayed onset muscle soreness is possible, with symptoms appearing within 24 hours and subsiding within 48 hours. Double-sided tape is used on the skin in this research. If you are sensitive or allergic to adhesives, please notify Christopher Moore.

\section{Alternatives}

You do not have to participate in this study. Participation is voluntary and you may drop out at any time without penalty.

\section{Benefits}

You may not receive any direct benefit from this study. If you are a WVU student, you will not receive any extra credit. The knowledge gained from this study may eventually benefit others by providing more knowledge on the shoulder joint and how it is effected by physical activity.

\section{Financial Considerations}

You will be compensated with a \$40 VISA gift card at successful completion of the data collection session.

\begin{tabular}{r|l} 
& Chestnut Ridge Research Building \\
Phone: 304-293-7073 & 886 Chestnut Ridge Road \\
Fax: 304-293-3098 & PO Box 6845 \\
http://oric.research.wvu.edu & Morgantown, WV 26506-6845
\end{tabular}

Chestnut Ridge Research Building

Morgantown, WV 26506-6845

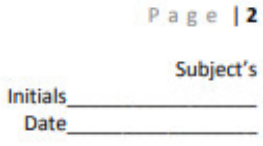




\section{Voluntary Compensation}

If you are injured as a result of this research, treatment will be available. Responsibility for this treatment will be borne by you. In the event that you are physically injured as a result of participating in this research, care will be available. You will, however, be responsible for the charges for the care. There is no commitment to provide any compensation for research-related injury. You should realize, however, that you have not released this institution from liability for negligence. Please contact the investigator, Ashish Nimbarte at 304-293-9473 if you are injured or for further information.

\section{Confidentiality}

Any information about you that is obtained as a result of your participation in this research will be kept as confidential as legally possible. Your research records and test results, just like hospital records, may be subpoenaed by court order or may be inspected by the study sponsor or federal regulatory authorities (including the FDA if applicable) without your additional consent.

In addition, there are certain instances where the researcher is legally required to give information to the appropriate authorities. These would include mandatory reporting of infectious diseases, mandatory reporting of information about behavior that is imminently dangerous to your child or to others, such as suicide, child abuse, etc.

In any publications that result from this research, neither your name nor any information from which you might be identified will be published without your consent.

\section{Voluntary Participation}

Participation in this study is voluntary. You are free to withdraw your consent to participate in this study at any time.

Refusal to participate or withdrawal will not affect your class standing or grades, and will involve no penalty to you. Refusal to participate or withdrawal will not affect your future care, or your employee status at West Virginia University.

In the event new information becomes available that may affect your willingness to participate in this study, this information will be given to you so that you can make an informed decision about whether or not to continue your participation.

You have been given the opportunity to ask questions about the research, and you have received answers concerning areas you did not understand.

Upon signing this form, you will receive a copy.

I willingly consent to participate in this research.

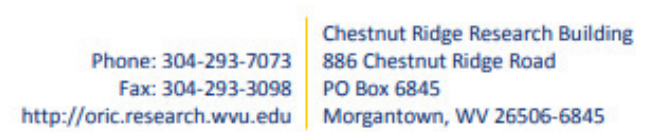

Phone: 304-293-7073

http://oric.research.wvu.edu

886 Chestnut Ridge Road

Morgantown, WV 26506-6845

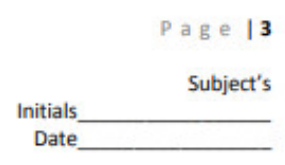




\section{Signatures}

Signature of Subject

\begin{tabular}{lll}
\hline Printed Name & Date & Time \\
\hline
\end{tabular}

The participant has had the opportunity to have questions addressed. The participant willingly agrees to be in the study.

Signature of Investigator or Co-Investigator

\begin{tabular}{lll}
\hline Printed Name & Date & Time
\end{tabular}

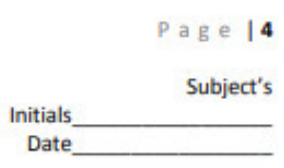


Appendix G: Borg CR-10 Rating of Perceived Exertion Scale

\begin{tabular}{|c|l|}
\hline \multicolumn{2}{|c|}{ Borg CR-10 Scale (1982) } \\
\hline 0 & Nothing at All \\
\hline 1 & Very Weak \\
\hline 2 & Weak \\
\hline 3 & Moderate \\
\hline 4 & Somewhat Strong \\
\hline 5 & Strong \\
\hline 6 & \\
\hline 7 & Very Strong \\
\hline 8 & \\
\hline 9 & Extremely Strong \\
\hline 10 & Maximum \\
\hline
\end{tabular}




\section{Appendix H: Stability modifier values for each $1^{\circ}$ increment}

\begin{tabular}{|c|c|c|c|c|c|}
\hline $\begin{array}{l}\text { Superior to } \\
\text { Anterior }\left({ }^{\circ}\right)\end{array}$ & $\begin{array}{l}\text { Unscaled } \\
\text { Value }\end{array}$ & $\begin{array}{c}\text { Scaled Stability } \\
\text { Modifier }\end{array}$ & $\begin{array}{l}\text { Superior to } \\
\text { Anterior }\left({ }^{\circ}\right)\end{array}$ & $\begin{array}{l}\text { Unscaled } \\
\text { Value }\end{array}$ & $\begin{array}{c}\text { Scaled Stability } \\
\text { Modifier }\end{array}$ \\
\hline $0^{\circ}$ & 59.0000 & 0.9219 & $45^{\circ}$ & 38.0000 & 0.5938 \\
\hline $1^{\circ}$ & 58.9719 & 0.9214 & $46^{\circ}$ & 37.8841 & 0.5919 \\
\hline $2^{\circ}$ & 58.8892 & 0.9201 & $47^{\circ}$ & 37.7697 & 0.5902 \\
\hline $3^{\circ}$ & 58.7542 & 0.9180 & $48^{\circ}$ & 37.6569 & 0.5884 \\
\hline $4^{\circ}$ & 58.5695 & 0.9151 & $49^{\circ}$ & 37.5457 & 0.5867 \\
\hline $5^{\circ}$ & 58.3374 & 0.9115 & $50^{\circ}$ & 37.4362 & 0.5849 \\
\hline $6^{\circ}$ & 58.0604 & 0.9072 & $51^{\circ}$ & 37.3284 & 0.5833 \\
\hline $7^{\circ}$ & 57.7409 & 0.9022 & $52^{\circ}$ & 37.2225 & 0.5816 \\
\hline $8^{\circ}$ & 57.3813 & 0.8966 & $53^{\circ}$ & 37.1183 & 0.5800 \\
\hline $9^{\circ}$ & 56.9840 & 0.8904 & $54^{\circ}$ & 37.0160 & 0.5784 \\
\hline $10^{\circ}$ & 56.5514 & 0.8836 & $55^{\circ}$ & 36.9156 & 0.5768 \\
\hline $11^{\circ}$ & 56.0860 & 0.8763 & $56^{\circ}$ & 36.8173 & 0.5753 \\
\hline $12^{\circ}$ & 55.5902 & 0.8686 & $57^{\circ}$ & 36.7209 & 0.5738 \\
\hline $13^{\circ}$ & 55.0664 & 0.8604 & $58^{\circ}$ & 36.6266 & 0.5723 \\
\hline $14^{\circ}$ & 54.5170 & 0.8518 & $59^{\circ}$ & 36.5344 & 0.5709 \\
\hline $15^{\circ}$ & 53.9444 & 0.8429 & $60^{\circ}$ & 36.4444 & 0.5694 \\
\hline $16^{\circ}$ & 53.3511 & 0.8336 & $61^{\circ}$ & 36.3567 & 0.5681 \\
\hline $17^{\circ}$ & 52.7395 & 0.8241 & $62^{\circ}$ & 36.2712 & 0.5667 \\
\hline $18^{\circ}$ & 52.1120 & 0.8143 & $63^{\circ}$ & 36.1880 & 0.5654 \\
\hline $19^{\circ}$ & 51.4710 & 0.8042 & $64^{\circ}$ & 36.1072 & 0.5642 \\
\hline $20^{\circ}$ & 50.8189 & 0.7940 & $65^{\circ}$ & 36.0288 & 0.5630 \\
\hline $21^{\circ}$ & 50.1582 & 0.7837 & $66^{\circ}$ & 35.9529 & 0.5618 \\
\hline $22^{\circ}$ & 49.4913 & 0.7733 & $67^{\circ}$ & 35.8795 & 0.5606 \\
\hline $23^{\circ}$ & 48.8206 & 0.7628 & $68^{\circ}$ & 35.8087 & 0.5595 \\
\hline $24^{\circ}$ & 48.1484 & 0.7523 & $69^{\circ}$ & 35.7404 & 0.5584 \\
\hline $25^{\circ}$ & 47.4774 & 0.7418 & $70^{\circ}$ & 35.6749 & 0.5574 \\
\hline $26^{\circ}$ & 46.8097 & 0.7314 & $71^{\circ}$ & 35.6121 & 0.5564 \\
\hline $27^{\circ}$ & 46.1480 & 0.7211 & $72^{\circ}$ & 35.5520 & 0.5555 \\
\hline $28^{\circ}$ & 45.4946 & 0.7109 & $73^{\circ}$ & 35.4947 & 0.5546 \\
\hline $29^{\circ}$ & 44.8518 & 0.7008 & $74^{\circ}$ & 35.4404 & 0.5538 \\
\hline $30^{\circ}$ & 44.2222 & 0.6910 & $75^{\circ}$ & 35.3889 & 0.5530 \\
\hline $31^{\circ}$ & 43.6082 & 0.6814 & $76^{\circ}$ & 35.3404 & 0.5522 \\
\hline $32^{\circ}$ & 43.0121 & 0.6721 & $77^{\circ}$ & 35.2949 & 0.5515 \\
\hline $33^{\circ}$ & 42.4364 & 0.6631 & $78^{\circ}$ & 35.2524 & 0.5508 \\
\hline $34^{\circ}$ & 41.8836 & 0.6544 & $79^{\circ}$ & 35.2131 & 0.5502 \\
\hline $35^{\circ}$ & 41.3560 & 0.6462 & $80^{\circ}$ & 35.1770 & 0.5496 \\
\hline $36^{\circ}$ & 40.8560 & 0.6384 & $81^{\circ}$ & 35.1440 & 0.5491 \\
\hline $37^{\circ}$ & 40.3861 & 0.6310 & $82^{\circ}$ & 35.1143 & 0.5487 \\
\hline $38^{\circ}$ & 39.9487 & 0.6242 & $83^{\circ}$ & 35.0879 & 0.5482 \\
\hline $39^{\circ}$ & 39.5462 & 0.6179 & $84^{\circ}$ & 35.0649 & 0.5479 \\
\hline $40^{\circ}$ & 39.1811 & 0.6122 & $85^{\circ}$ & 35.0453 & 0.5476 \\
\hline $41^{\circ}$ & 38.8557 & 0.6071 & $86^{\circ}$ & 35.0291 & 0.5473 \\
\hline $42^{\circ}$ & 38.5724 & 0.6027 & $87^{\circ}$ & 35.0164 & 0.5471 \\
\hline $43^{\circ}$ & 38.3338 & 0.5990 & $88^{\circ}$ & 35.0073 & 0.5470 \\
\hline $44^{\circ}$ & 38.1422 & 0.5960 & $89^{\circ}$ & 35.0018 & 0.5469 \\
\hline
\end{tabular}




\begin{tabular}{|c|c|c|c|c|c|}
\hline $\begin{array}{l}\text { Anterior to } \\
\text { Inferior }\left({ }^{\circ}\right)\end{array}$ & $\begin{array}{c}\text { Unscaled } \\
\text { Value }\end{array}$ & $\begin{array}{c}\text { Scaled Stability } \\
\text { Modifier }\end{array}$ & $\begin{array}{l}\text { Anterior to } \\
\text { Inferior }\left({ }^{\circ}\right)\end{array}$ & $\begin{array}{c}\text { Unscaled } \\
\text { Value }\end{array}$ & $\begin{array}{c}\text { Scaled Stability } \\
\text { Modifier }\end{array}$ \\
\hline $90^{\circ}$ & 35.0000 & 0.5469 & $135^{\circ}$ & 46.0000 & 0.7188 \\
\hline $91^{\circ}$ & 35.0095 & 0.5470 & $136^{\circ}$ & 46.3164 & 0.7237 \\
\hline $92^{\circ}$ & 35.0375 & 0.5475 & $137^{\circ}$ & 46.6577 & 0.7290 \\
\hline $93^{\circ}$ & 35.0835 & 0.5482 & $138^{\circ}$ & 47.0223 & 0.7347 \\
\hline $94^{\circ}$ & 35.1470 & 0.5492 & $139^{\circ}$ & 47.4090 & 0.7408 \\
\hline $95^{\circ}$ & 35.2274 & 0.5504 & $140^{\circ}$ & 47.8161 & 0.7471 \\
\hline $96^{\circ}$ & 35.3241 & 0.5519 & $141^{\circ}$ & 48.2422 & 0.7538 \\
\hline $97^{\circ}$ & 35.4367 & 0.5537 & $142^{\circ}$ & 48.6859 & 0.7607 \\
\hline $98^{\circ}$ & 35.5645 & 0.5557 & $143^{\circ}$ & 49.1456 & 0.7679 \\
\hline $99^{\circ}$ & 35.7070 & 0.5579 & $144^{\circ}$ & 49.6199 & 0.7753 \\
\hline $100^{\circ}$ & 35.8637 & 0.5604 & $145^{\circ}$ & 50.1073 & 0.7829 \\
\hline $101^{\circ}$ & 36.0340 & 0.5630 & $146^{\circ}$ & 50.6063 & 0.7907 \\
\hline $102^{\circ}$ & 36.2174 & 0.5659 & $147^{\circ}$ & 51.1156 & 0.7987 \\
\hline $103^{\circ}$ & 36.4133 & 0.5690 & $148^{\circ}$ & 51.6335 & 0.8068 \\
\hline $104^{\circ}$ & 36.6211 & 0.5722 & $149^{\circ}$ & 52.1587 & 0.8150 \\
\hline $105^{\circ}$ & 36.8404 & 0.5756 & $150^{\circ}$ & 52.6897 & 0.8233 \\
\hline $106^{\circ}$ & 37.0705 & 0.5792 & $151^{\circ}$ & 53.2249 & 0.8316 \\
\hline $107^{\circ}$ & 37.3109 & 0.5830 & $152^{\circ}$ & 53.7629 & 0.8400 \\
\hline $108^{\circ}$ & 37.5611 & 0.5869 & $153^{\circ}$ & 54.3023 & 0.8485 \\
\hline $109^{\circ}$ & 37.8205 & 0.5909 & $154^{\circ}$ & 54.8416 & 0.8569 \\
\hline $110^{\circ}$ & 38.0886 & 0.5951 & $155^{\circ}$ & 55.3793 & 0.8653 \\
\hline $111^{\circ}$ & 38.3648 & 0.5994 & $156^{\circ}$ & 55.9139 & 0.8737 \\
\hline $112^{\circ}$ & 38.6486 & 0.6039 & $157^{\circ}$ & 56.4440 & 0.8819 \\
\hline $113^{\circ}$ & 38.9393 & 0.6084 & $158^{\circ}$ & 56.9681 & 0.8901 \\
\hline $114^{\circ}$ & 39.2366 & 0.6131 & $159^{\circ}$ & 57.4847 & 0.8982 \\
\hline $115^{\circ}$ & 39.5398 & 0.6178 & $160^{\circ}$ & 57.9923 & 0.9061 \\
\hline $116^{\circ}$ & 39.8483 & 0.6226 & $161^{\circ}$ & 58.4896 & 0.9139 \\
\hline $117^{\circ}$ & 40.1617 & 0.6275 & $162^{\circ}$ & 58.9749 & 0.9215 \\
\hline $118^{\circ}$ & 40.4793 & 0.6325 & $163^{\circ}$ & 59.4469 & 0.9289 \\
\hline $119^{\circ}$ & 40.8006 & 0.6375 & $164^{\circ}$ & 59.9040 & 0.9360 \\
\hline $120^{\circ}$ & 41.1252 & 0.6426 & $165^{\circ}$ & 60.3448 & 0.9429 \\
\hline $121^{\circ}$ & 41.4523 & 0.6477 & $166^{\circ}$ & 60.7679 & 0.9495 \\
\hline $122^{\circ}$ & 41.7815 & 0.6528 & $167^{\circ}$ & 61.1717 & 0.9558 \\
\hline $123^{\circ}$ & 42.1123 & 0.6580 & $168^{\circ}$ & 61.5548 & 0.9618 \\
\hline $124^{\circ}$ & 42.4440 & 0.6632 & $169^{\circ}$ & 61.9156 & 0.9674 \\
\hline $125^{\circ}$ & 42.7761 & 0.6684 & $170^{\circ}$ & 62.2529 & 0.9727 \\
\hline $126^{\circ}$ & 43.1081 & 0.6736 & $171^{\circ}$ & 62.5650 & 0.9776 \\
\hline $127^{\circ}$ & 43.4395 & 0.6787 & $172^{\circ}$ & 62.8505 & 0.9820 \\
\hline $128^{\circ}$ & 43.7696 & 0.6839 & $173^{\circ}$ & 63.1079 & 0.9861 \\
\hline $129^{\circ}$ & 44.0979 & 0.6890 & $174^{\circ}$ & 63.3357 & 0.9896 \\
\hline $130^{\circ}$ & 44.4240 & 0.6941 & $175^{\circ}$ & 63.5326 & 0.9927 \\
\hline $131^{\circ}$ & 44.7471 & 0.6992 & $176^{\circ}$ & 63.6969 & 0.9953 \\
\hline $132^{\circ}$ & 45.0668 & 0.7042 & $177^{\circ}$ & 63.8273 & 0.9973 \\
\hline $133^{\circ}$ & 45.3826 & 0.7091 & $178^{\circ}$ & 63.9223 & 0.9988 \\
\hline $134^{\circ}$ & 45.6938 & 0.7140 & $179^{\circ}$ & 63.9803 & 0.9997 \\
\hline
\end{tabular}




\begin{tabular}{|c|c|c|c|c|c|}
\hline $\begin{array}{c}\text { Inferior to } \\
\text { Posterior }\left({ }^{\circ}\right)\end{array}$ & $\begin{array}{c}\text { Unscaled } \\
\text { Value }\end{array}$ & $\begin{array}{c}\text { Scaled Stability } \\
\text { Modifier }\end{array}$ & $\begin{array}{c}\text { Inferior to } \\
\text { Posterior }\left({ }^{\circ}\right)\end{array}$ & $\begin{array}{c}\text { Unscaled } \\
\text { Value }\end{array}$ & $\begin{array}{c}\text { Scaled Stability } \\
\text { Modifier }\end{array}$ \\
\hline $180^{\circ}$ & 64.0000 & 1.0000 & $225^{\circ}$ & 50.0000 & 0.7813 \\
\hline $181^{\circ}$ & 63.9870 & 0.9998 & $226^{\circ}$ & 49.6490 & 0.7758 \\
\hline $182^{\circ}$ & 63.9485 & 0.9992 & $227^{\circ}$ & 49.2791 & 0.7700 \\
\hline $183^{\circ}$ & 63.8853 & 0.9982 & $228^{\circ}$ & 48.8917 & 0.7639 \\
\hline $184^{\circ}$ & 63.7984 & 0.9968 & $229^{\circ}$ & 48.4879 & 0.7576 \\
\hline $185^{\circ}$ & 63.6884 & 0.9951 & $230^{\circ}$ & 48.0690 & 0.7511 \\
\hline $186^{\circ}$ & 63.5563 & 0.9931 & $231^{\circ}$ & 47.6362 & 0.7443 \\
\hline $187^{\circ}$ & 63.4029 & 0.9907 & $232^{\circ}$ & 47.1907 & 0.7374 \\
\hline $188^{\circ}$ & 63.2289 & 0.9880 & $233^{\circ}$ & 46.7337 & 0.7302 \\
\hline $189^{\circ}$ & 63.0354 & 0.9849 & $234^{\circ}$ & 46.2666 & 0.7229 \\
\hline $190^{\circ}$ & 62.8230 & 0.9816 & $235^{\circ}$ & 45.7904 & 0.7155 \\
\hline $191^{\circ}$ & 62.5926 & 0.9780 & $236^{\circ}$ & 45.3065 & 0.7079 \\
\hline $192^{\circ}$ & 62.3450 & 0.9741 & $237^{\circ}$ & 44.8161 & 0.7003 \\
\hline $193^{\circ}$ & 62.0812 & 0.9700 & $238^{\circ}$ & 44.3203 & 0.6925 \\
\hline $194^{\circ}$ & 61.8018 & 0.9657 & $239^{\circ}$ & 43.8205 & 0.6847 \\
\hline $195^{\circ}$ & 61.5078 & 0.9611 & $240^{\circ}$ & 43.3178 & 0.6768 \\
\hline $196^{\circ}$ & 61.1999 & 0.9562 & $241^{\circ}$ & 42.8135 & 0.6690 \\
\hline $197^{\circ}$ & 60.8791 & 0.9512 & $242^{\circ}$ & 42.3088 & 0.6611 \\
\hline $198^{\circ}$ & 60.5461 & 0.9460 & $243^{\circ}$ & 41.8049 & 0.6532 \\
\hline $199^{\circ}$ & 60.2017 & 0.9407 & $244^{\circ}$ & 41.3031 & 0.6454 \\
\hline $200^{\circ}$ & 59.8469 & 0.9351 & $245^{\circ}$ & 40.8045 & 0.6376 \\
\hline $201^{\circ}$ & 59.4824 & 0.9294 & $246^{\circ}$ & 40.3105 & 0.6299 \\
\hline $202^{\circ}$ & 59.1091 & 0.9236 & $247^{\circ}$ & 39.8222 & 0.6222 \\
\hline $203^{\circ}$ & 58.7277 & 0.9176 & $248^{\circ}$ & 39.3409 & 0.6147 \\
\hline $204^{\circ}$ & 58.3392 & 0.9116 & $249^{\circ}$ & 38.8678 & 0.6073 \\
\hline $205^{\circ}$ & 57.9444 & 0.9054 & $250^{\circ}$ & 38.4041 & 0.6001 \\
\hline $206^{\circ}$ & 57.5441 & 0.8991 & $251^{\circ}$ & 37.9511 & 0.5930 \\
\hline $207^{\circ}$ & 57.1391 & 0.8928 & $252^{\circ}$ & 37.5099 & 0.5861 \\
\hline $208^{\circ}$ & 56.7303 & 0.8864 & $253^{\circ}$ & 37.0819 & 0.5794 \\
\hline $209^{\circ}$ & 56.3184 & 0.8800 & $254^{\circ}$ & 36.6682 & 0.5729 \\
\hline $210^{\circ}$ & 55.9044 & 0.8735 & $255^{\circ}$ & 36.2700 & 0.5667 \\
\hline $211^{\circ}$ & 55.4891 & 0.8670 & $256^{\circ}$ & 35.8886 & 0.5608 \\
\hline $212^{\circ}$ & 55.0732 & 0.8605 & $257^{\circ}$ & 35.5253 & 0.5551 \\
\hline $213^{\circ}$ & 54.6577 & 0.8540 & $258^{\circ}$ & 35.1812 & 0.5497 \\
\hline $214^{\circ}$ & 54.2433 & 0.8476 & $259^{\circ}$ & 34.8576 & 0.5446 \\
\hline $215^{\circ}$ & 53.8310 & 0.8411 & $260^{\circ}$ & 34.5556 & 0.5399 \\
\hline $216^{\circ}$ & 53.4214 & 0.8347 & $261^{\circ}$ & 34.2766 & 0.5356 \\
\hline $217^{\circ}$ & 53.0155 & 0.8284 & $262^{\circ}$ & 34.0218 & 0.5316 \\
\hline $218^{\circ}$ & 52.6141 & 0.8221 & $263^{\circ}$ & 33.7923 & 0.5280 \\
\hline $219^{\circ}$ & 52.2181 & 0.8159 & $264^{\circ}$ & 33.5895 & 0.5248 \\
\hline $220^{\circ}$ & 51.8281 & 0.8098 & $265^{\circ}$ & 33.4145 & 0.5221 \\
\hline $221^{\circ}$ & 51.4452 & 0.8038 & $266^{\circ}$ & 33.2685 & 0.5198 \\
\hline $222^{\circ}$ & 51.0701 & 0.7980 & $267^{\circ}$ & 33.1529 & 0.5180 \\
\hline $223^{\circ}$ & 50.7036 & 0.7922 & $268^{\circ}$ & 33.0688 & 0.5167 \\
\hline $224^{\circ}$ & 50.3467 & 0.7867 & $269^{\circ}$ & 33.0174 & 0.5159 \\
\hline
\end{tabular}




\begin{tabular}{|c|c|c|c|c|c|}
\hline $\begin{array}{l}\text { Posterior to } \\
\text { Superior }\left({ }^{\circ}\right)\end{array}$ & $\begin{array}{l}\text { Unscaled } \\
\text { Value }\end{array}$ & $\begin{array}{c}\text { Scaled Stability } \\
\text { Modifier }\end{array}$ & $\begin{array}{l}\text { Posterior to } \\
\text { Superior }\left({ }^{\circ}\right)\end{array}$ & $\begin{array}{c}\text { Unscaled } \\
\text { Value }\end{array}$ & $\begin{array}{c}\text { Scaled Stability } \\
\text { Modifier }\end{array}$ \\
\hline $270^{\circ}$ & 33.0000 & 0.5156 & $315^{\circ}$ & 40.0000 & 0.6250 \\
\hline $271^{\circ}$ & 33.0053 & 0.5157 & $316^{\circ}$ & 40.2451 & 0.6288 \\
\hline $272^{\circ}$ & 33.0209 & 0.5160 & $317^{\circ}$ & 40.5244 & 0.6332 \\
\hline $273^{\circ}$ & 33.0467 & 0.5164 & $318^{\circ}$ & 40.8362 & 0.6381 \\
\hline $274^{\circ}$ & 33.0824 & 0.5169 & $319^{\circ}$ & 41.1786 & 0.6434 \\
\hline $275^{\circ}$ & 33.1278 & 0.5176 & $320^{\circ}$ & 41.5498 & 0.6492 \\
\hline $276^{\circ}$ & 33.1825 & 0.5185 & $321^{\circ}$ & 41.9479 & 0.6554 \\
\hline $277^{\circ}$ & 33.2464 & 0.5195 & $322^{\circ}$ & 42.3711 & 0.6620 \\
\hline $278^{\circ}$ & 33.3192 & 0.5206 & $323^{\circ}$ & 42.8176 & 0.6690 \\
\hline $279^{\circ}$ & 33.4006 & 0.5219 & $324^{\circ}$ & 43.2855 & 0.6763 \\
\hline $280^{\circ}$ & 33.4905 & 0.5233 & $325^{\circ}$ & 43.7731 & 0.6840 \\
\hline $281^{\circ}$ & 33.5884 & 0.5248 & $326^{\circ}$ & 44.2785 & 0.6919 \\
\hline $282^{\circ}$ & 33.6943 & 0.5265 & $327^{\circ}$ & 44.7999 & 0.7000 \\
\hline $283^{\circ}$ & 33.8079 & 0.5282 & $328^{\circ}$ & 45.3354 & 0.7084 \\
\hline $284^{\circ}$ & 33.9289 & 0.5301 & $329^{\circ}$ & 45.8833 & 0.7169 \\
\hline $285^{\circ}$ & 34.0570 & 0.5321 & $330^{\circ}$ & 46.4416 & 0.7256 \\
\hline $286^{\circ}$ & 34.1920 & 0.5343 & $331^{\circ}$ & 47.0086 & 0.7345 \\
\hline $287^{\circ}$ & 34.3337 & 0.5365 & $332^{\circ}$ & 47.5824 & 0.7435 \\
\hline $288^{\circ}$ & 34.4818 & 0.5388 & $333^{\circ}$ & 48.1612 & 0.7525 \\
\hline $289^{\circ}$ & 34.6361 & 0.5412 & $334^{\circ}$ & 48.7432 & 0.7616 \\
\hline $290^{\circ}$ & 34.7963 & 0.5437 & $335^{\circ}$ & 49.3266 & 0.7707 \\
\hline $291^{\circ}$ & 34.9622 & 0.5463 & $336^{\circ}$ & 49.9094 & 0.7798 \\
\hline $292^{\circ}$ & 35.1335 & 0.5490 & $337^{\circ}$ & 50.4900 & 0.7889 \\
\hline $293^{\circ}$ & 35.3100 & 0.5517 & $338^{\circ}$ & 51.0664 & 0.7979 \\
\hline $294^{\circ}$ & 35.4914 & 0.5546 & $339^{\circ}$ & 51.6369 & 0.8068 \\
\hline $295^{\circ}$ & 35.6775 & 0.5575 & $340^{\circ}$ & 52.1995 & 0.8156 \\
\hline $296^{\circ}$ & 35.8681 & 0.5604 & $341^{\circ}$ & 52.7526 & 0.8243 \\
\hline $297^{\circ}$ & 36.0628 & 0.5635 & $342^{\circ}$ & 53.2942 & 0.8327 \\
\hline $298^{\circ}$ & 36.2614 & 0.5666 & $343^{\circ}$ & 53.8225 & 0.8410 \\
\hline $299^{\circ}$ & 36.4637 & 0.5697 & $344^{\circ}$ & 54.3357 & 0.8490 \\
\hline $300^{\circ}$ & 36.6695 & 0.5730 & $345^{\circ}$ & 54.8319 & 0.8567 \\
\hline $301^{\circ}$ & 36.8785 & 0.5762 & $346^{\circ}$ & 55.3094 & 0.8642 \\
\hline $302^{\circ}$ & 37.0904 & 0.5795 & $347^{\circ}$ & 55.7663 & 0.8713 \\
\hline $303^{\circ}$ & 37.3050 & 0.5829 & $348^{\circ}$ & 56.2008 & 0.8781 \\
\hline $304^{\circ}$ & 37.5220 & 0.5863 & $349^{\circ}$ & 56.6110 & 0.8845 \\
\hline $305^{\circ}$ & 37.7413 & 0.5897 & $350^{\circ}$ & 56.9951 & 0.8905 \\
\hline $306^{\circ}$ & 37.9625 & 0.5932 & $351^{\circ}$ & 57.3514 & 0.8961 \\
\hline $307^{\circ}$ & 38.1854 & 0.5966 & $352^{\circ}$ & 57.6779 & 0.9012 \\
\hline $308^{\circ}$ & 38.4097 & 0.6002 & $353^{\circ}$ & 57.9728 & 0.9058 \\
\hline $309^{\circ}$ & 38.6353 & 0.6037 & $354^{\circ}$ & 58.2344 & 0.9099 \\
\hline $310^{\circ}$ & 38.8618 & 0.6072 & $355^{\circ}$ & 58.4607 & 0.9134 \\
\hline $311^{\circ}$ & 39.0890 & 0.6108 & $356^{\circ}$ & 58.6500 & 0.9164 \\
\hline $312^{\circ}$ & 39.3167 & 0.6143 & $357^{\circ}$ & 58.8004 & 0.9188 \\
\hline $313^{\circ}$ & 39.5446 & 0.6179 & $358^{\circ}$ & 58.9101 & 0.9205 \\
\hline $314^{\circ}$ & 39.7724 & 0.6214 & $359^{\circ}$ & 58.9772 & 0.9215 \\
\hline
\end{tabular}




\section{Appendix I: Best Sets Regression Analysis of 11 Potential Sets of Predictors}

Best Subsets Regression (Set 1): +Fwd/-Bwd, +Right/-Left, +Up/-Down

\begin{tabular}{ccccccccc}
\hline Vars & R-Sq & R-Sq (adj) & $\begin{array}{c}\text { R-Sq } \\
\text { (pred) }\end{array}$ & $\begin{array}{c}\text { Mallows } \\
\text { Cp }\end{array}$ & S & +Fwd/-Bwd & +Right/-Left & +Up/-Down \\
\hline 1 & 34.8 & 34.6 & 34.0 & 245.9 & 13.557 & & $X$ \\
1 & 32.6 & 32.4 & 31.9 & 265.5 & 13.779 & $X$ & & \\
2 & 61.8 & 61.5 & 61.0 & 2.1 & 10.394 & $X$ & $X$ & $X$ \\
2 & 37.9 & 37.6 & 36.8 & 218.9 & 13.242 & & $X$ & $X$ \\
3 & 61.8 & 61.4 & 60.8 & 4.0 & 10.408 & $X$ & $X$ & $X$ \\
\hline
\end{tabular}

Best Subsets Regression (Set 2): Forward, Backward, Right, Left, Up, Down

\begin{tabular}{|c|c|c|c|c|c|c|c|c|c|c|c|}
\hline Vars & $R-S q$ & $R-S q$ (adj) & $\begin{array}{c}\text { R-Sq } \\
\text { (pred) }\end{array}$ & $\begin{array}{c}\text { Mallows } \\
\text { Cp }\end{array}$ & $\mathbf{S}$ & Forward & Backward & Right & Left & Up & Down \\
\hline 1 & 33.0 & 32.9 & 32.4 & 352.5 & 13.734 & & & & & & $X$ \\
\hline 1 & 33.0 & 32.8 & 32.3 & 352.7 & 13.736 & $x$ & & & & & \\
\hline 2 & 56.0 & 55.7 & 55.0 & 114.5 & 11.151 & $x$ & & & & $x$ & \\
\hline 2 & 55.7 & 55.4 & 55.0 & 117.8 & 11.192 & $x$ & & & & & $x$ \\
\hline 3 & 62.0 & 61.7 & 61.1 & 53.6 & 10.376 & $x$ & & & & $x$ & $x$ \\
\hline 3 & 58.1 & 57.8 & 56.9 & 94.0 & 10.891 & $x$ & & & $x$ & $x$ & \\
\hline 4 & 64.2 & 63.8 & 62.9 & 32.6 & 10.086 & $x$ & & $x$ & $x$ & $x$ & \\
\hline 4 & 63.8 & 63.4 & 62.6 & 37.0 & 10.146 & $x$ & & & $x$ & $x$ & $x$ \\
\hline 5 & 67.0 & 66.5 & 65.7 & 5.7 & 9.704 & $x$ & & $x$ & $x$ & $X$ & $x$ \\
\hline
\end{tabular}


Best Subsets Regression (Set 3): Norm +Fwd/-Bwd, Norm +Right/-Left, Norm +Up/-Down

\begin{tabular}{ccccccccc}
\hline Vars & R-Sq & R-Sq (adj) & $\begin{array}{c}\text { R-Sq } \\
\text { (pred) }\end{array}$ & $\begin{array}{c}\text { Mallows } \\
\text { Cp }\end{array}$ & S & $\begin{array}{c}\text { Norm } \\
\text { +Fwd/Bwd }\end{array}$ & $\begin{array}{c}\text { Norm } \\
\text { +Right/-Left }\end{array}$ & $\begin{array}{c}\text { Norm +Up/- } \\
\text { Down }\end{array}$ \\
\hline 1 & 34.7 & 34.5 & 33.9 & 241.6 & 13.566 & & $X$ \\
1 & 32.5 & 32.3 & 31.8 & 261.5 & 13.793 & $X$ & & $X$ \\
2 & 61.4 & 61.2 & 60.7 & 2.1 & 10.438 & $X$ & $X$ & $X$ \\
2 & 37.9 & 37.5 & 36.8 & 214.9 & 13.251 & & $X$ & $X$ \\
3 & 61.4 & 61.1 & 60.5 & 4.0 & 10.452 & $X$ & $X$ & $X$ \\
\hline
\end{tabular}

Best Subsets Regression (Set 4): Norm Fwd, Norm Bwd, Norm Right, Norm Left, Norm Up, Norm Down

\begin{tabular}{|c|c|c|c|c|c|c|c|c|c|c|c|}
\hline Vars & R-Sq & R-Sq (adj) & $\begin{array}{c}\text { R-Sq } \\
\text { (pred) }\end{array}$ & $\begin{array}{c}\text { Mallows } \\
\text { Cp }\end{array}$ & $\mathbf{S}$ & Norm Fwd & Norm Bwd & Norm Right & $\begin{array}{l}\text { Norm } \\
\text { Left }\end{array}$ & $\begin{array}{l}\text { Norm } \\
\text { Up }\end{array}$ & $\begin{array}{l}\text { Norm } \\
\text { Down }\end{array}$ \\
\hline 1 & 33.2 & 33.0 & 32.6 & 339.3 & 13.715 & & & & & & $x$ \\
\hline 1 & 32.9 & 32.7 & 32.2 & 342.8 & 13.750 & $x$ & & & & & \\
\hline 2 & 55.6 & 55.3 & 54.9 & 111.3 & 11.203 & $x$ & & & & & $x$ \\
\hline 2 & 55.4 & 55.2 & 54.5 & 112.8 & 11.221 & $x$ & & & & $x$ & \\
\hline 3 & 61.7 & 61.3 & 60.7 & 50.7 & 10.423 & $x$ & & & & $x$ & $x$ \\
\hline 3 & 57.5 & 57.1 & 56.3 & 93.5 & 10.973 & $x$ & & & & $x$ & \\
\hline 4 & 63.5 & 63.1 & 62.3 & 33.5 & 10.181 & $x$ & & $x$ & $x$ & $x$ & \\
\hline 4 & 63.3 & 62.9 & 62.2 & 35.3 & 10.205 & $x$ & & & $x$ & $x$ & $x$ \\
\hline 5 & 66.4 & 65.9 & 65.2 & 5.7 & 9.783 & $x$ & & $x$ & $x$ & $x$ & $x$ \\
\hline 5 & 63.7 & 63.2 & 62.3 & 33.9 & 10.173 & $x$ & $x$ & $x$ & $x$ & $x$ & \\
\hline 6 & 66.5 & 65.9 & 65.2 & 7.0 & 9.787 & $x$ & $x$ & $x$ & $x$ & $x$ & $\mathrm{X}$ \\
\hline
\end{tabular}


Best Subsets Regression (Set 5): SQRT(+Fwd/-Bwd), SQRT(+Right/-Left), SQRT(+Up/-Down)

\begin{tabular}{ccccccccc}
\hline Vars & R-Sq & R-Sq (adj) & $\begin{array}{c}\text { R-Sq } \\
\text { (pred) }\end{array}$ & $\begin{array}{c}\text { Mallows } \\
\text { Cp }\end{array}$ & S & $\begin{array}{c}\text { SQRT(+Fwd/- } \\
\text { Bwd) }\end{array}$ & $\begin{array}{c}\text { SQRT(+Left/- } \\
\text { Right) }\end{array}$ & $\begin{array}{c}\text { SQRT(+Up/- } \\
\text { Down) }\end{array}$ \\
\hline 1 & 35.4 & 35.2 & 34.6 & 159.1 & 13.490 & & & $X$ \\
1 & 22.9 & 22.7 & 22.1 & 256.8 & 14.733 & $X$ & & \\
2 & 55.5 & 55.2 & 54.6 & 3.6 & 11.217 & $X$ & $X$ \\
2 & 38.6 & 38.2 & 37.5 & 136.2 & 13.174 & & $X$ & $X$ \\
3 & 55.7 & 55.3 & 54.6 & 4.0 & 11.208 & $X$ & $X$ & $X$ \\
\hline
\end{tabular}

Best Subsets Regression (Set 6): SQRT(Fwd), SQRT(Bwd), SQRT(Right), SQRT(Left), SQRT(Up), SQRT(Down)

\begin{tabular}{|c|c|c|c|c|c|c|c|c|c|c|c|}
\hline Vars & $\mathrm{R}-\mathrm{Sq}$ & R-Sq (adj) & $\begin{array}{c}\text { R-Sq } \\
\text { (pred) }\end{array}$ & $\begin{array}{c}\text { Mallows } \\
\text { Cp }\end{array}$ & S & SQRT(Fwd) & SQRT(Bwd) & SQRT(Right) & SQRT(Left) & SQRT(Up) & SQRT(Down) \\
\hline 1 & 34.4 & 34.2 & 33.7 & 251.1 & 13.595 & & & & & & $\mathrm{X}$ \\
\hline 1 & 28.0 & 27.8 & 27.1 & 309.2 & 14.239 & & & & & $x$ & \\
\hline 2 & 54.2 & 53.9 & 53.5 & 72.2 & 11.375 & $x$ & & & & & $x$ \\
\hline 2 & 54.1 & 53.8 & 53.1 & 73.5 & 11.393 & $x$ & & & & $x$ & \\
\hline 3 & 57.7 & 57.4 & 56.7 & 41.9 & 10.942 & $x$ & & & & $x$ & $x$ \\
\hline 3 & 55.9 & 55.5 & 54.8 & 59.0 & 11.182 & $x$ & $x$ & & & $x$ & \\
\hline 4 & 59.0 & 58.5 & 57.9 & 32.7 & 10.799 & $x$ & $x$ & & & $x$ & $x$ \\
\hline 4 & 58.6 & 58.1 & 57.3 & 36.3 & 10.851 & $x$ & & & $x$ & $x$ & $x$ \\
\hline 5 & 60.7 & 60.1 & 59.2 & 19.1 & 10.586 & $x$ & $x$ & $x$ & $x$ & $x$ & \\
\hline 5 & 60.4 & 59.8 & 58.9 & 22.0 & 10.629 & $x$ & & $x$ & $x$ & $x$ & $x$ \\
\hline 6 & 62.2 & 61.6 & 60.7 & 7.0 & 10.391 & $x$ & $x$ & $x$ & $x$ & $x$ & $x$ \\
\hline
\end{tabular}


Best Subsets Regression (Set 7): ABS(+Fwd/-Bwd), ABS(+Right/-Left), ABS(+Up/-Down)

\begin{tabular}{ccccccccc}
\hline Vars & R-Sq & R-Sq (adj) & $\begin{array}{c}\text { R-Sq } \\
\text { (pred) }\end{array}$ & $\begin{array}{c}\text { Mallows } \\
\text { Cp }\end{array}$ & S & $\begin{array}{c}\text { ABS(+Fwd/- } \\
\text { Bwd) }\end{array}$ & $\begin{array}{c}\text { ABS(+Right/- } \\
\text { Left) }\end{array}$ & $\begin{array}{c}\text { ABSS(+Up/- } \\
\text { Down) }\end{array}$ \\
\hline 1 & 33.2 & 33.0 & 32.5 & 22.8 & 13.715 & $\mathrm{X}$ & & \\
1 & 3.2 & 2.9 & 2.1 & 189.7 & 16.516 & & & $\mathrm{X}$ \\
2 & 37.3 & 36.9 & 36.4 & 2.2 & 13.310 & $\mathrm{X}$ & $\mathrm{X}$ & \\
2 & 33.4 & 33.0 & 32.4 & 24.0 & 13.720 & $\mathrm{X}$ & & $\mathrm{X}$ \\
3 & 37.3 & 36.8 & 36.1 & 4.0 & 13.325 & $\mathrm{X}$ & $\mathrm{X}$ & $\mathrm{X}$ \\
\hline
\end{tabular}

Best Subsets Regression (Set 8): (+Fwd/-Bwd)^2, (+Right/-Left)^2, (+Up/-Down)^2

\begin{tabular}{ccccccccc}
\hline Vars & R-Sq & R-Sq (adj) & $\begin{array}{c}\text { R-Sq } \\
\text { (pred) }\end{array}$ & $\begin{array}{c}\text { Mallows } \\
\text { Cp }\end{array}$ & S & $\begin{array}{c}\text { (+Fwd/- } \\
\text { Bwd)^2 }\end{array}$ & $\begin{array}{c}\text { (+Right/- } \\
\text { Left)^2 }\end{array}$ & $\begin{array}{c}\text { (+Up/- } \\
\text { Down)^2 }\end{array}$ \\
\hline 1 & 34.4 & 34.2 & 33.7 & 13.1 & 13.592 & $\mathrm{X}$ & & \\
1 & 4.2 & 3.9 & 3.2 & 179.7 & 16.432 & & & $\mathrm{X}$ \\
2 & 36.8 & 36.4 & 35.9 & 2.0 & 13.362 & $\mathrm{X}$ & $\mathrm{X}$ & \\
2 & 34.6 & 34.3 & 33.6 & 13.9 & 13.590 & $\mathrm{X}$ & & $\mathrm{X}$ \\
3 & 36.8 & 36.3 & 35.6 & 4.0 & 13.382 & $\mathrm{X}$ & $\mathrm{X}$ & $\mathrm{X}$ \\
\hline
\end{tabular}


Best Subsets Regression (Set 9): $F w^{\wedge} 2, B^{\wedge}$ 2, Right^2, Left^2, Up^2, Down^2

\begin{tabular}{|c|c|c|c|c|c|c|c|c|c|c|c|}
\hline Vars & $\mathrm{R}-\mathrm{Sq}$ & R-Sq (adj) & $\begin{array}{c}\text { R-Sq } \\
\text { (pred) }\end{array}$ & $\begin{array}{c}\text { Mallows } \\
\text { Cp }\end{array}$ & $\mathbf{S}$ & $F_{w d \wedge} 2$ & $B w d^{\wedge} 2$ & Right^2 & Left^2 & $\mathrm{Up}^{\wedge} 2$ & Down^2 \\
\hline 1 & 34.5 & 34.3 & 33.7 & 257.1 & 13.589 & $x$ & & & & & \\
\hline 1 & 26.6 & 26.4 & 26.0 & 329.3 & 14.376 & & & & & & $x$ \\
\hline 2 & 50.6 & 50.4 & 49.9 & 109.7 & 11.809 & $x$ & & & & & $x$ \\
\hline 2 & 49.9 & 49.6 & 48.7 & 116.5 & 11.897 & $x$ & & & & $x$ & \\
\hline 3 & 57.7 & 57.4 & 56.6 & 46.1 & 10.942 & $x$ & & & & $x$ & $X$ \\
\hline 3 & 53.3 & 52.9 & 51.9 & 86.8 & 11.499 & $x$ & & & $X$ & $x$ & \\
\hline 4 & 60.5 & 60.0 & 59.1 & 22.8 & 10.596 & $x$ & & & $x$ & $x$ & $x$ \\
\hline 4 & 58.0 & 57.5 & 56.7 & 45.5 & 10.921 & $x$ & & $x$ & & $x$ & $x$ \\
\hline 5 & 62.4 & 61.9 & 60.9 & 7.0 & 10.350 & $x$ & & $x$ & $x$ & $x$ & $x$ \\
\hline 5 & 60.5 & 60.0 & 59.1 & 24.4 & 10.605 & $x$ & $x$ & & $x$ & $x$ & $x$ \\
\hline 6 & 62.6 & 62.0 & 61.1 & 7.0 & 10.334 & $x$ & $x$ & $x$ & $x$ & $x$ & $x$ \\
\hline
\end{tabular}

Best Subsets Regression (Set 10): (+Fwd/-Bwd)^3, (+Right/-Left)^3, (+Up/-Down)^3

\begin{tabular}{ccccccccc}
\hline Vars & R-Sq & R-Sq (adj) & $\begin{array}{c}\text { R-Sq } \\
\text { (pred) }\end{array}$ & $\begin{array}{c}\text { Mallows } \\
\text { Cp }\end{array}$ & S & $\begin{array}{c}\text { (+Fwd/- } \\
\text { Bwd)^3 }\end{array}$ & $\begin{array}{c}\text { (+Right/- } \\
\text { Left)^3 }\end{array}$ & $\begin{array}{c}\text { (+Up/- } \\
\text { Down)^3 }\end{array}$ \\
\hline 1 & 32.4 & 32.2 & 31.6 & 126.8 & 13.803 & $X$ & & \\
1 & 20.7 & 20.5 & 19.8 & 208.4 & 14.942 & & & $X$ \\
2 & 50.4 & 50.1 & 49.4 & 2.5 & 11.842 & $X$ & & $X$ \\
2 & 32.9 & 32.5 & 31.9 & 125.3 & 13.771 & $X$ & $X$ & \\
3 & 50.4 & 50.0 & 49.3 & 4.0 & 11.851 & $X$ & $X$ & $X$ \\
\hline
\end{tabular}


Best Subsets Regression (Set 11): Fwd^3, Bwd^3, Right^3, Left^3, Up^3, Down^3

\begin{tabular}{|c|c|c|c|c|c|c|c|c|c|c|c|}
\hline Vars & $\mathrm{R}-\mathrm{Sq}$ & R-Sq (adj) & $\begin{array}{c}\text { R-Sq } \\
\text { (pred) }\end{array}$ & $\begin{array}{c}\text { Mallows } \\
\text { Cp }\end{array}$ & S & $F_{w d \wedge 3}$ & $B w d^{\wedge} 3$ & Right^3 & Left^3 & Up^3 & Down^3 \\
\hline 1 & 32.4 & 32.2 & 31.6 & 162.0 & 13.804 & $x$ & & & & & \\
\hline 1 & 21.1 & 20.9 & 20.4 & 247.1 & 14.910 & & & & & & $x$ \\
\hline 2 & 45.0 & 44.7 & 44.2 & 68.8 & 12.466 & $x$ & & & & & $x$ \\
\hline 2 & 42.1 & 41.8 & 40.8 & 90.4 & 12.787 & $x$ & & & & $x$ & \\
\hline 3 & 50.4 & 50.0 & 49.1 & 30.1 & 11.856 & $x$ & & & & $x$ & $X$ \\
\hline 3 & 47.0 & 46.6 & 45.9 & 55.5 & 12.251 & $x$ & & & $X$ & & $x$ \\
\hline 4 & 52.9 & 52.4 & 51.4 & 12.9 & 11.564 & $x$ & & & $x$ & $x$ & $x$ \\
\hline 4 & 50.7 & 50.2 & 49.4 & 29.5 & 11.832 & $x$ & $x$ & & & $x$ & $x$ \\
\hline 5 & 53.8 & 53.2 & 52.1 & 8.2 & 11.471 & $x$ & & $x$ & $x$ & $x$ & $x$ \\
\hline 5 & 53.2 & 52.5 & 51.6 & 13.2 & 11.553 & $x$ & $x$ & & $x$ & $x$ & $x$ \\
\hline 6 & 54.2 & 53.5 & 52.5 & 7.0 & 11.435 & $x$ & $x$ & $x$ & $x$ & $x$ & $x$ \\
\hline
\end{tabular}




\section{Appendix J1: Minitab Output from Regression Analysis (Regression Equation \#1)}

Regression Analysis: Composite Index versus Forward, Right, Left, Up, Down, SQRT(Up), SQRT(Down)

Stepwise Selection of Terms

$\alpha$ to enter $=0.15, \alpha$ to remove $=0.15$

The stepwise procedure added terms during the procedure in order to maintain a hierarchical model at each step.

Analysis of Variance

$\begin{array}{lrrrrr}\text { Source } & \text { DF } & \text { Adj SS } & \text { Adj MS } & \text { F-Value } & \text { P-Value } \\ \text { Regression } & 16 & 77021.9 & 4813.87 & 74.74 & 0.000 \\ \quad \text { Forward } & 1 & 1532.2 & 1532.22 & 23.79 & 0.000 \\ \quad \text { Right } & 1 & 4416.4 & 4416.35 & 68.57 & 0.000 \\ \quad \text { Left } & 1 & 5247.1 & 5247.12 & 81.47 & 0.000 \\ \text { Up } & 1 & 587.6 & 587.55 & 9.12 & 0.003 \\ \text { Down } & 1 & 363.6 & 363.55 & 5.64 & 0.018 \\ \text { SQRT(Up) } & 1 & 616.4 & 616.39 & 9.57 & 0.002 \\ \text { SQRT(Down) } & 1 & 408.3 & 408.29 & 6.34 & 0.012 \\ \quad \text { Forward*Forward } & 1 & 153.0 & 152.98 & 2.38 & 0.124 \\ \text { Up*Up } & 1 & 1060.1 & 1060.05 & 16.46 & 0.000 \\ \text { Forward*Right } & 1 & 1439.6 & 1439.62 & 22.35 & 0.000 \\ \quad \text { Forward*Up } & 1 & 257.6 & 257.56 & 4.00 & 0.046 \\ \quad \text { Forward*Down } & 1 & 587.3 & 587.29 & 9.12 & 0.003 \\ \quad \text { Right*SQRT(Up) } & 1 & 1679.1 & 1679.08 & 26.07 & 0.000 \\ \quad \text { Left*SQRT(Down) } & 1 & 257.2 & 257.15 & 3.99 & 0.047 \\ \quad \text { Up*SQRT(Up) } & 1 & 843.1 & 843.11 & 13.09 & 0.000 \\ \quad \text { Down*SQRT(Down) } & 1 & 362.3 & 362.33 & 5.63 & 0.018 \\ \text { Error } & 335 & 21575.6 & 64.40 & & \\ \text { Total } & 351 & 98597.5 & & & \end{array}$

Model Summary

$\begin{array}{rrll}S & R-s q & R-s q(\text { adj }) & R-s q(\text { pred) } \\ 8.02526 & 78.12 \% & 77.07 \% & 75.71 \%\end{array}$

Coefficients

$\begin{array}{lrrrrr}\text { Term } & \text { Coef } & \text { SE Coef } & \text { T-Value } & \text { P-Value } & \text { VIF } \\ \text { Constant } & -19.02 & 5.09 & -3.74 & 0.000 & \\ \text { Forward } & 2.445 & 0.501 & 4.88 & 0.000 & 43.99 \\ \text { Right } & 1.559 & 0.188 & 8.28 & 0.000 & 9.50 \\ \text { Left } & 2.374 & 0.263 & 9.03 & 0.000 & 3.12 \\ \text { Up } & -27.53 & 9.11 & -3.02 & 0.003 & 15006.78 \\ \text { Down } & -6.74 & 2.84 & -2.38 & 0.018 & 1787.55 \\ \text { SQRT(Up) } & 33.6 & 10.9 & 3.09 & 0.002 & 1530.40 \\ \text { SQRT(Down) } & 15.51 & 6.16 & 2.52 & 0.012 & 569.17 \\ \text { Forward*Forward } & 0.0255 & 0.0165 & 1.54 & 0.124 & 20.52\end{array}$




$\begin{array}{lrrrrr}\text { Up*Up } & -1.250 & 0.308 & -4.06 & 0.000 & 4590.15 \\ \text { Forward*Right } & -0.0736 & 0.0156 & -4.73 & 0.000 & 5.71 \\ \text { Forward*Up } & -0.0457 & 0.0229 & -2.00 & 0.046 & 9.44 \\ \text { Forward*Down } & -0.0555 & 0.0184 & -3.02 & 0.003 & 6.64 \\ \text { Right*SQRT(Up) } & -0.2791 & 0.0547 & -5.11 & 0.000 & 5.67 \\ \text { Left*SQRT(Down) } & -0.207 & 0.104 & -2.00 & 0.047 & 2.78 \\ \text { Up*SQRT(Up) } & 10.49 & 2.90 & 3.62 & 0.000 & 23942.90 \\ \text { Down*SQRT(Down) } & 0.895 & 0.377 & 2.37 & 0.018 & 514.96\end{array}$

Regression Equation

Composite Index $=-19.02+2.445$ Forward +1.559 Right +2.374 Left -27.53 Up

- 6.74 Down + 33.6 SQRT(Up) + 15.51 SQRT(Down) + 0.0255 Forward*Forward - 1.250 Up*Up

- 0.0736 Forward*Right - 0.0457 Forward*Up - 0.0555 Forward*Down

- 0.2791 Right*SQRT(Up) - 0.207 Left*SQRT(Down) + 10.49 Up*SQRT(Up)

+0.895 Down*SQRT(Down) 


\title{
Appendix J2: Minitab Output from Regression Analysis (Regression Equation \#2)
}

Regression Analysis: Composite Index versus Forward, Right, Left, Up, Down, SQRT(Up), SQRT(Down)

\author{
Stepwise Selection of Terms \\ $\alpha$ to enter $=0.15, \alpha$ to remove $=0.15$
}

Analysis of Variance

$\begin{array}{lrrrrr}\text { Source } & \text { DF } & \text { Adj SS } & \text { Adj MS } & \text { F-Value } & \text { P-Value } \\ \text { Regression } & 8 & 76053.0 & 9506.6 & 144.64 & 0.000 \\ \quad \text { SQRT(Down) } & 1 & 1702.3 & 1702.3 & 25.90 & 0.000 \\ \quad \text { Forward*Forward } & 1 & 27561.8 & 27561.8 & 419.33 & 0.000 \\ \text { Right*Right } & 1 & 1169.3 & 1169.3 & 17.79 & 0.000 \\ \text { Up*Up } & 1 & 4911.6 & 4911.6 & 74.73 & 0.000 \\ \quad \text { Forward*Left } & 1 & 6302.6 & 6302.6 & 95.89 & 0.000 \\ \quad \text { Right*SQRT(Up) } & 1 & 1050.4 & 1050.4 & 15.98 & 0.000 \\ \quad \text { Right*SQRT(Down) } & 1 & 424.4 & 424.4 & 6.46 & 0.011 \\ \quad \text { Up*SQRT(Up) } & 1 & 6156.2 & 6156.2 & 93.66 & 0.000 \\ \text { Error } & 343 & 22544.6 & 65.7 & & \\ \text { Total } & 351 & 98597.5 & & & \end{array}$

Model Summary

$$
\begin{array}{rrrr}
S & R-s q & R-s q(a d j) & R-s q(\text { pred) } \\
8.10726 & 77.13 \% & 76.60 \% & 75.68 \%
\end{array}
$$

Coefficients

$\begin{array}{lrrrrr}\text { Term } & \text { Coef } & \text { SE Coef } & \text { T-Value } & \text { P-Value } & \text { VIF } \\ \text { Constant } & 12.41 & 1.64 & 7.58 & 0.000 & \\ \text { SQRT(Down) } & -2.597 & 0.510 & -5.09 & 0.000 & 3.83 \\ \text { Forward*Forward } & 0.08620 & 0.00421 & 20.48 & 0.000 & 1.31 \\ \text { Right*Right } & 0.02886 & 0.00684 & 4.22 & 0.000 & 4.44 \\ \text { Up*Up } & -0.3638 & 0.0421 & -8.64 & 0.000 & 83.98 \\ \text { Forward*Left } & 0.2004 & 0.0205 & 9.79 & 0.000 & 1.39 \\ \text { Right*SQRT(Up) } & -0.2110 & 0.0528 & -4.00 & 0.000 & 5.18 \\ \text { Right*SQRT(Down) } & 0.1262 & 0.0497 & 2.54 & 0.011 & 4.38 \\ \text { Up*SQRT(Up) } & 1.849 & 0.191 & 9.68 & 0.000 & 101.94\end{array}$

Regression Equation

Composite Index $=12.41-2.597 \mathrm{SQRT}($ Down $)+0.08620$ Forward*Forward

+0.02886 Right*Right -0.3638 Up*Up +0.2004 Forward*Left -0.2110 Right*SQRT(Up)

+0.1262 Right*SQRT(Down) + 1.849 Up*SQRT(Up) 


\title{
Appendix J3: Minitab Output from Regression Analysis (Regression Equation \#3)
}

Regression Analysis: Composite Index versus Forward, Right, Left, Up, Down, SQRT(Up), SQRT(Down)

\author{
Stepwise Selection of Terms \\ $\alpha$ to enter $=0.15, \alpha$ to remove $=0.15$
}

Analysis of Variance

$\begin{array}{lrrrrr}\text { Source } & \text { DF } & \text { Adj SS } & \text { Adj MS } & \text { F-Value } & \text { P-Value } \\ \text { Regression } & 9 & 74769.6 & 8307.7 & 119.24 & 0.000 \\ \quad \text { Forward } & 1 & 28880.3 & 28880.3 & 414.52 & 0.000 \\ \quad \text { Right } & 1 & 3562.9 & 3562.9 & 51.14 & 0.000 \\ \text { Left } & 1 & 3422.9 & 3422.9 & 49.13 & 0.000 \\ \text { SQRT(Up) } & 1 & 4337.8 & 4337.8 & 62.26 & 0.000 \\ \quad \text { Forward*Right } & 1 & 3259.5 & 3259.5 & 46.78 & 0.000 \\ \quad \text { Forward*Down } & 1 & 1579.6 & 1579.6 & 22.67 & 0.000 \\ \quad \text { Right*SQRT(Up) } & 1 & 148.4 & 148.4 & 2.13 & 0.145 \\ \quad \text { Right*SQRT(Down) } & 1 & 244.9 & 244.9 & 3.52 & 0.062 \\ \quad \text { Left*SQRT(Up) } & 1 & 336.7 & 336.7 & 4.83 & 0.029 \\ \text { Error } & 342 & 23827.9 & 69.7 & & \\ \text { Total } & 351 & 98597.5 & & & \end{array}$

Model Summary
$8.34699 \quad 75.83 \% \quad r 5.20 \% \quad r .18 \%$
$S \quad R-s q \quad R-s q(\operatorname{adj})$
$\mathrm{R}-\mathrm{sq}$ (pred)

Coefficients

$\begin{array}{lrrrrr}\text { Term } & \text { Coef } & \text { SE Coef } & \text { T-Value } & \text { P-Value } & \text { VIF } \\ \text { Constant } & -11.10 & 2.36 & -4.70 & 0.000 & \\ \text { Forward } & 2.996 & 0.147 & 20.36 & 0.000 & 3.50 \\ \text { Right } & 1.443 & 0.202 & 7.15 & 0.000 & 10.08 \\ \text { Left } & 1.796 & 0.256 & 7.01 & 0.000 & 2.74 \\ \text { SQRT(Up) } & 5.441 & 0.690 & 7.89 & 0.000 & 5.71 \\ \text { Forward*Right } & -0.0878 & 0.0128 & -6.84 & 0.000 & 3.59 \\ \text { Forward*Down } & -0.0518 & 0.0109 & -4.76 & 0.000 & 2.15 \\ \text { Right*SQRT(Up) } & -0.0971 & 0.0665 & -1.46 & 0.145 & 7.77 \\ \text { Right*SQRT(Down) } & 0.0898 & 0.0479 & 1.87 & 0.062 & 3.84 \\ \text { Left*SQRT(Up) } & 0.286 & 0.130 & 2.20 & 0.029 & 2.48\end{array}$

Regression Equation

Composite Index $=-11.10+2.996$ Forward +1.443 Right +1.796 Left +5.441 SQRT(Up)

- 0.0878 Forward*Right - 0.0518 Forward*Down - 0.0971 Right*SQRT(Up)

+0.0898 Right*SQRT(Down) + 0.286 Left*SQRT(Up) 


\title{
Appendix J4: Minitab Output from Regression Analysis (Regression Equation \#4)
}

Regression Analysis: Composite Index versus Forward, Right, Left, Up, Down, SQRT(Up), SQRT(Down)

\author{
Stepwise Selection of Terms \\ $\alpha$ to enter $=0.1, \alpha$ to remove $=0.1$
}

Analysis of Variance

$\begin{array}{lrrrrr}\text { Source } & \text { DF } & \text { Adj SS } & \text { Adj MS } & \text { F-Value } & \text { P-Value } \\ \text { Regression } & 8 & 74621.3 & 9327.7 & 133.44 & 0.000 \\ \quad \text { Forward } & 1 & 29694.5 & 29694.5 & 424.80 & 0.000 \\ \quad \text { Right } & 1 & 4775.8 & 4775.8 & 68.32 & 0.000 \\ \quad \text { Left } & 1 & 3289.1 & 3289.1 & 47.05 & 0.000 \\ \quad \text { SQRT(Up) } & 1 & 7228.7 & 7228.7 & 103.41 & 0.000 \\ \quad \text { Forward*Right } & 1 & 3145.7 & 3145.7 & 45.00 & 0.000 \\ \quad \text { Forward*Down } & 1 & 2544.3 & 2544.3 & 36.40 & 0.000 \\ \quad \text { Right*SQRT(Down) } & 1 & 864.7 & 864.7 & 12.37 & 0.000 \\ \quad \text { Left*SQRT(Up) } & 1 & 727.7 & 727.7 & 10.41 & 0.001 \\ \text { Error } & 343 & 23976.3 & 69.9 & & \\ \text { Total } & 351 & 98597.5 & & & \end{array}$

Model Summary
$\begin{array}{rr}S & R-S q \\ 8.36072 & 75.68 \%\end{array}$
$R-s q(\operatorname{adj})$
$\mathrm{R}-\mathrm{sq}$ (pred)
$8.36072 \quad 75.68 \% \quad 75.12 \% \quad 74.22 \%$

Coefficients

$\begin{array}{lrrrrr}\text { Term } & \text { Coef } & \text { SE Coef } & \text { T-Value } & \text { P-Value } & \text { VIF } \\ \text { Constant } & -10.15 & 2.28 & -4.46 & 0.000 & \\ \text { Forward } & 3.020 & 0.147 & 20.61 & 0.000 & 3.46 \\ \text { Right } & 1.247 & 0.151 & 8.27 & 0.000 & 5.62 \\ \text { Left } & 1.695 & 0.247 & 6.86 & 0.000 & 2.54 \\ \text { SQRT(Up) } & 4.692 & 0.461 & 10.17 & 0.000 & 2.55 \\ \text { Forward*Right } & -0.0858 & 0.0128 & -6.71 & 0.000 & 3.55 \\ \text { Forward*Down } & -0.05888 & 0.00976 & -6.03 & 0.000 & 1.72 \\ \text { Right*SQRT(Down) } & 0.1329 & 0.0378 & 3.52 & 0.000 & 2.38 \\ \text { Left*SQRT(Up) } & 0.373 & 0.116 & 3.23 & 0.001 & 1.96\end{array}$

Regression Equation

Composite Index $=-10.15+3.020$ Forward +1.247 Right +1.695 Left +4.692 SQRT(Up)

- 0.0858 Forward*Right -0.05888 Forward*Down +0.1329 Right*SQRT(Down)

+0.373 Left*SQRT(Up) 
Appendix K1. Predicted Strain with the Load 20 Inches Above Sternal Notch.

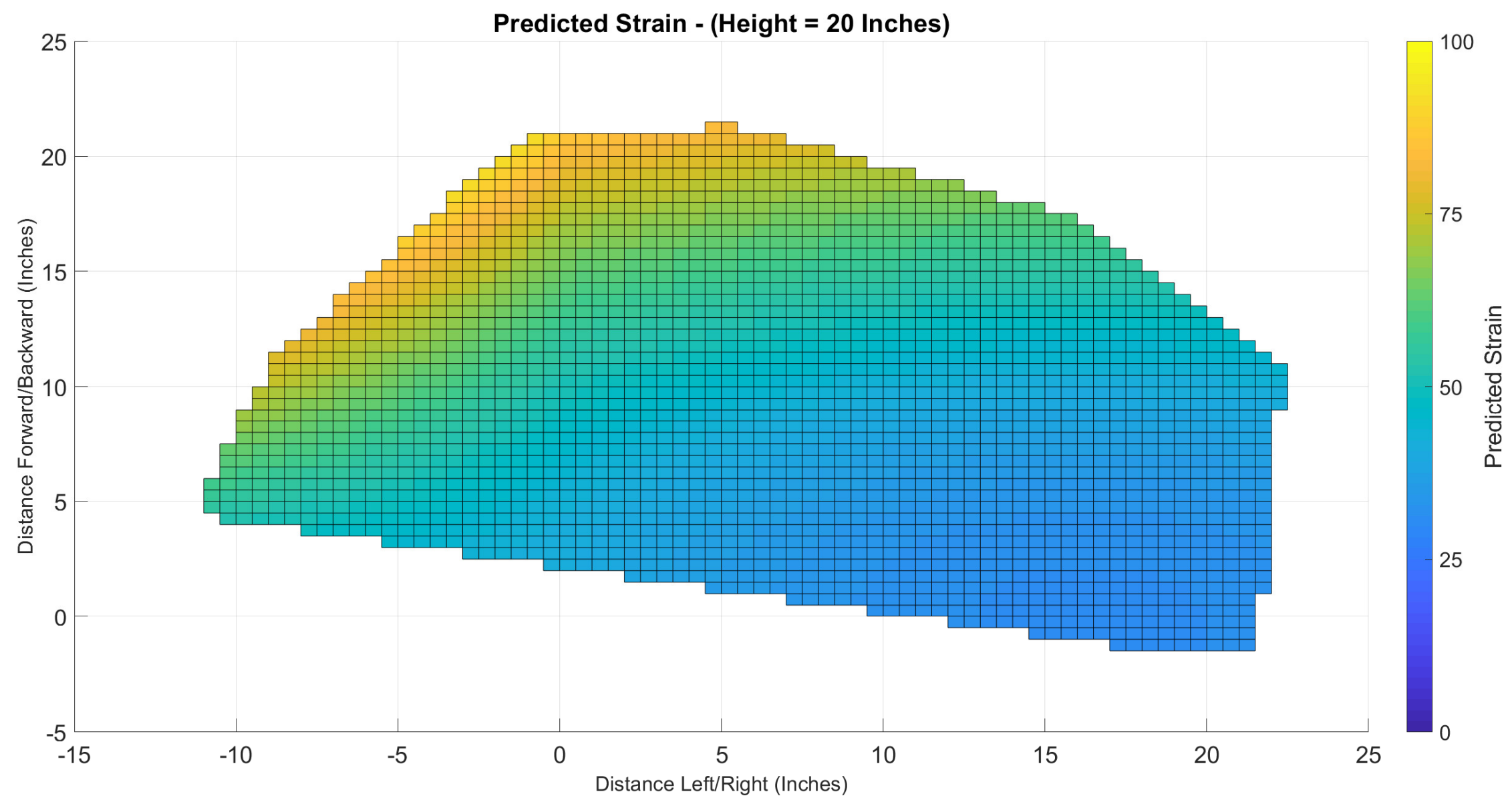


Appendix K2. Predicted Strain with the Load 15 Inches Above Sternal Notch.

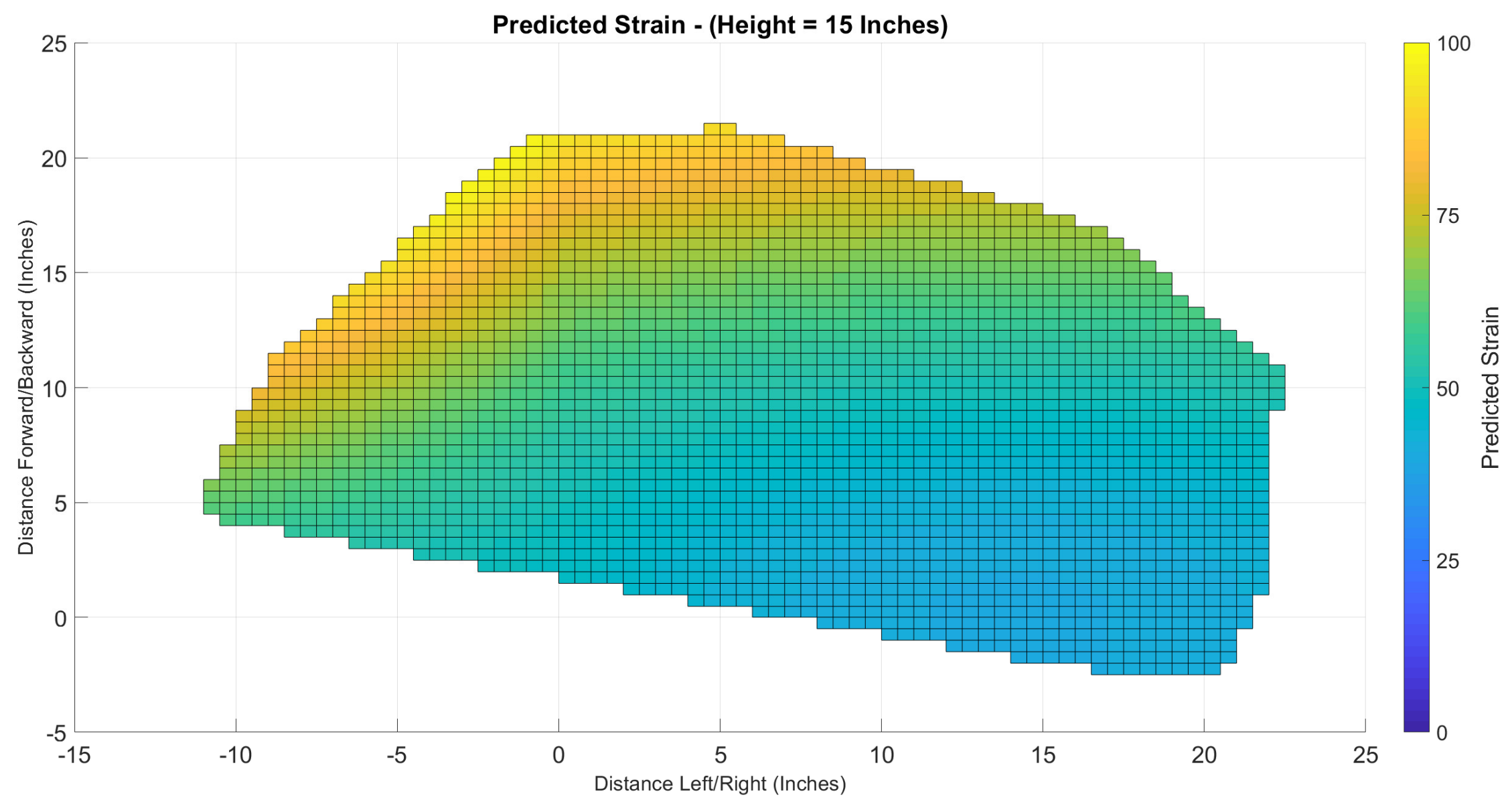


Appendix K3. Predicted Strain with the Load 10 Inches Above Sternal Notch.

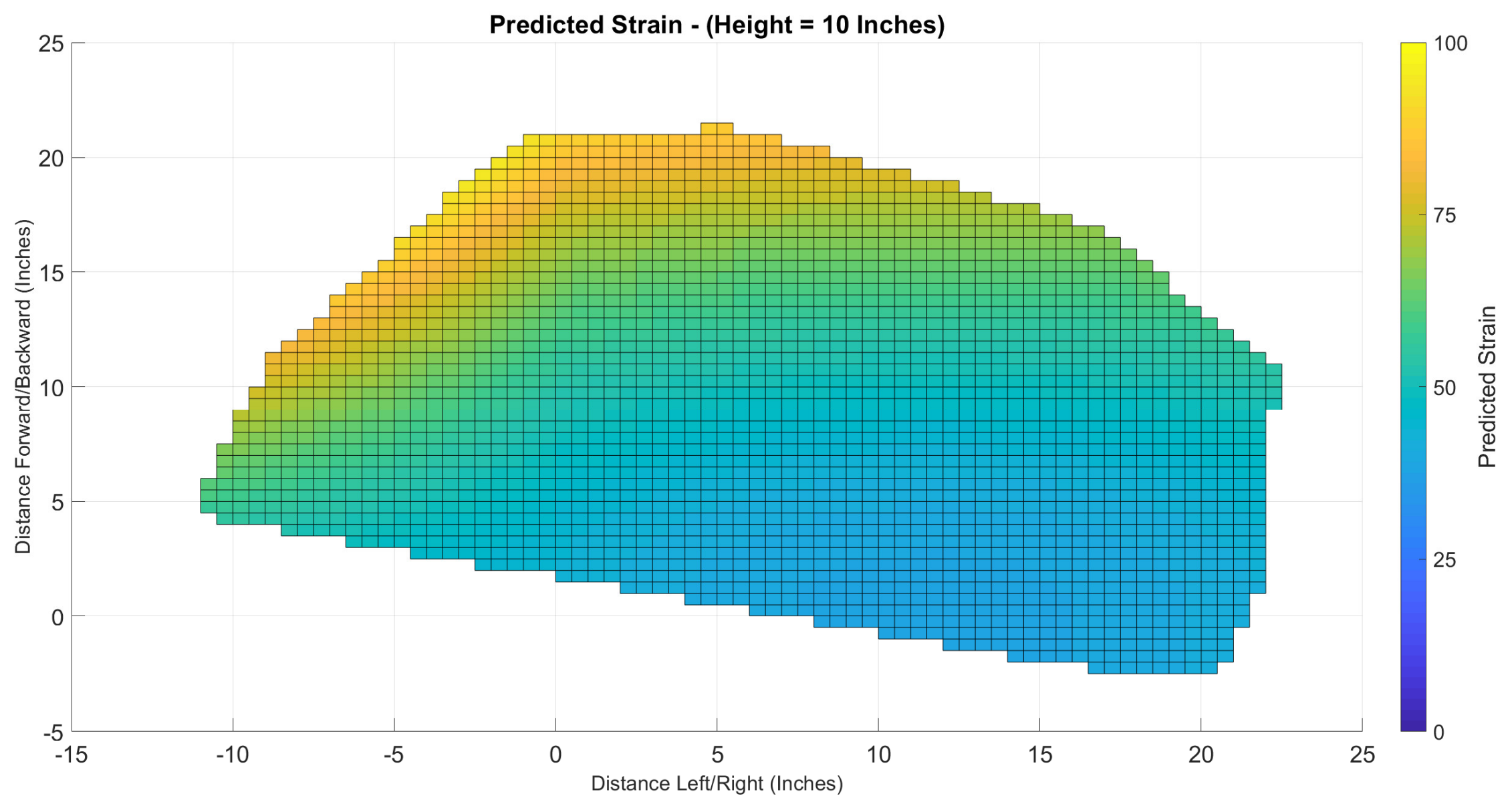


Appendix K4. Predicted Strain with the Load 5 Inches Above Sternal Notch.

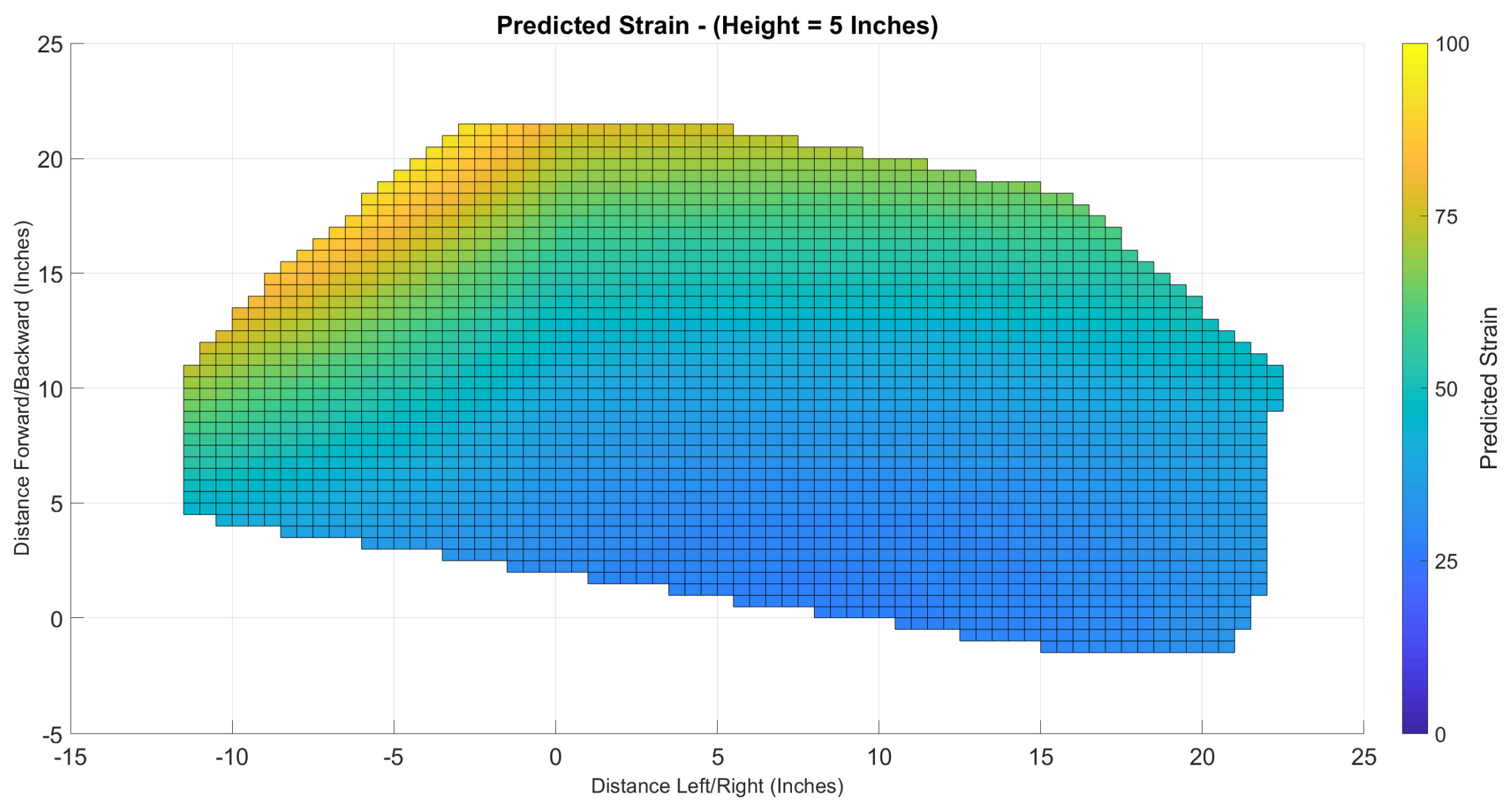


Appendix K5. Predicted Strain with the Load Horizontally Aligned with Sternal Notch.

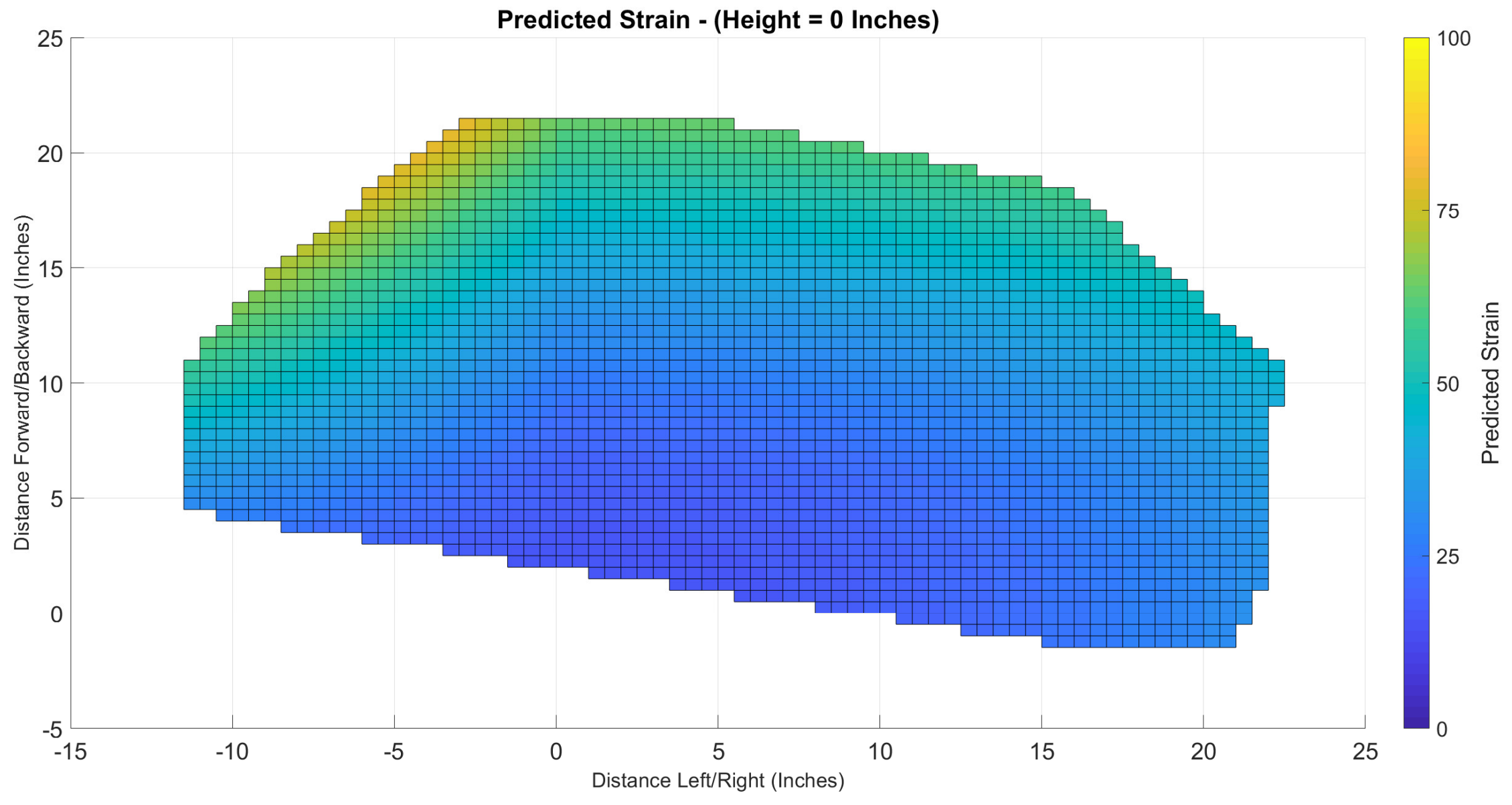


Appendix K6. Predicted Strain with the Load 5 Inches Below Sternal Notch.

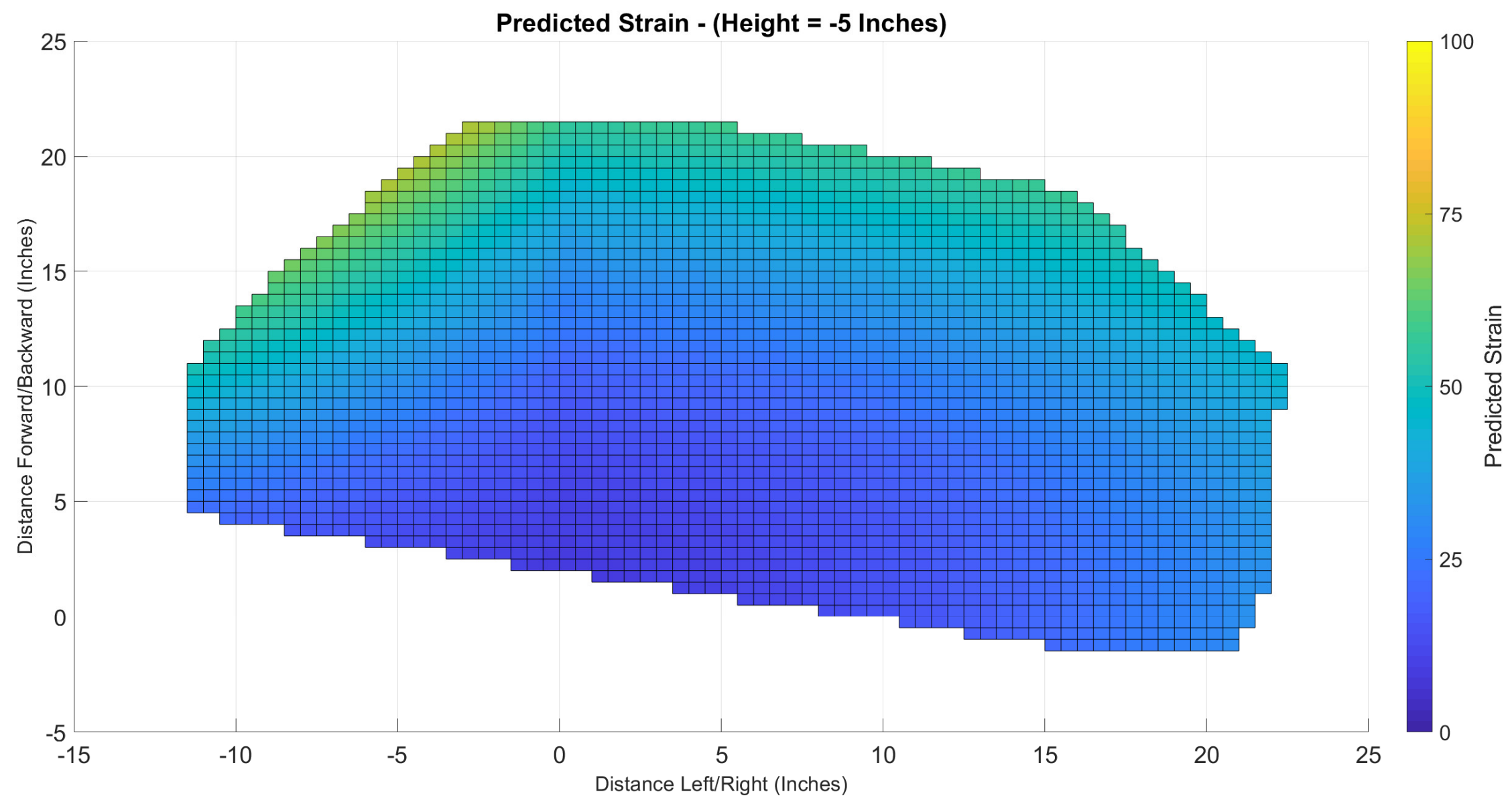


Appendix K7. Predicted Strain with the Load 10 Inches Below Sternal Notch.

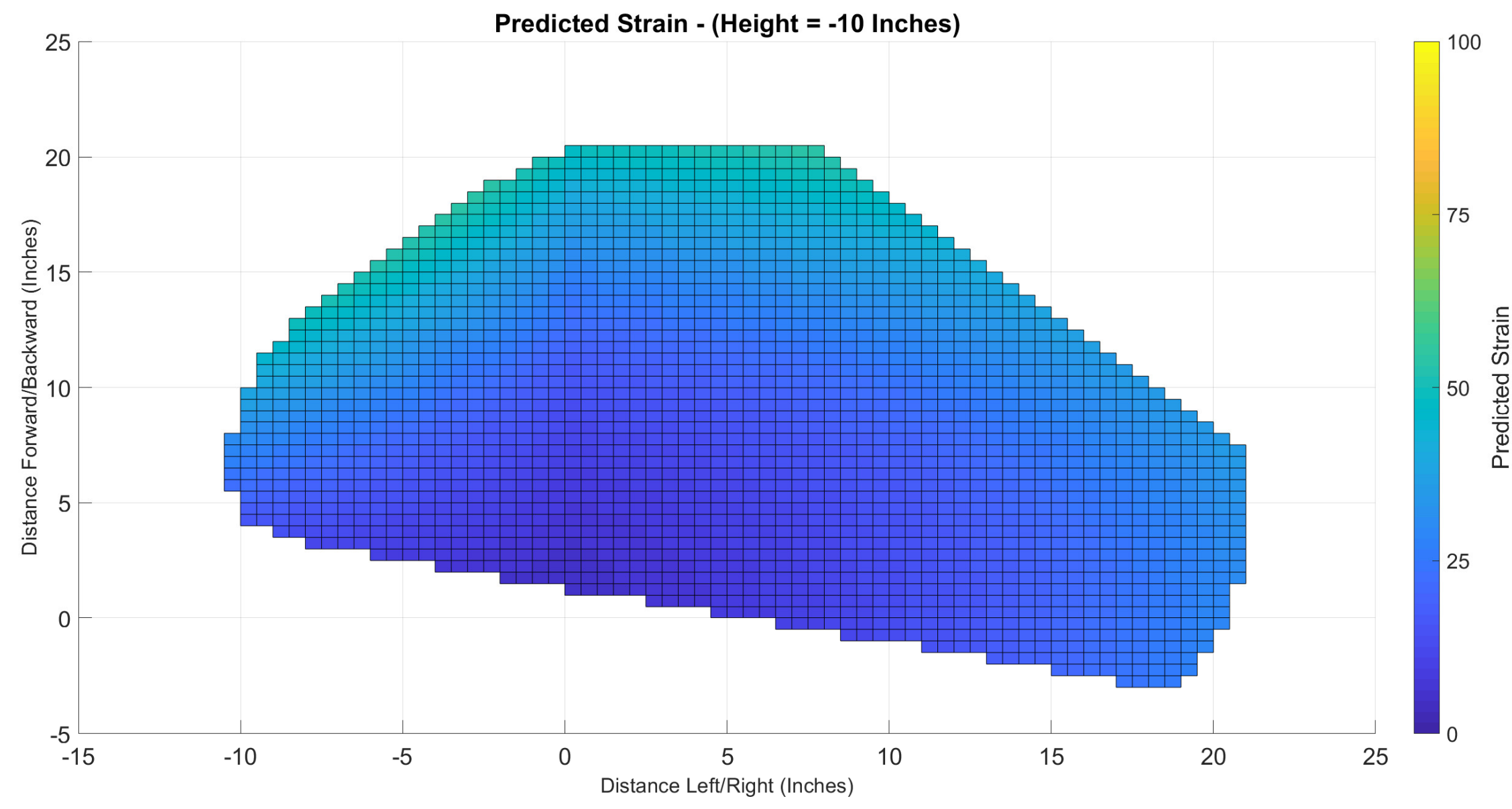


Appendix K8. Predicted Strain with the Load 15 Inches Below Sternal Notch.

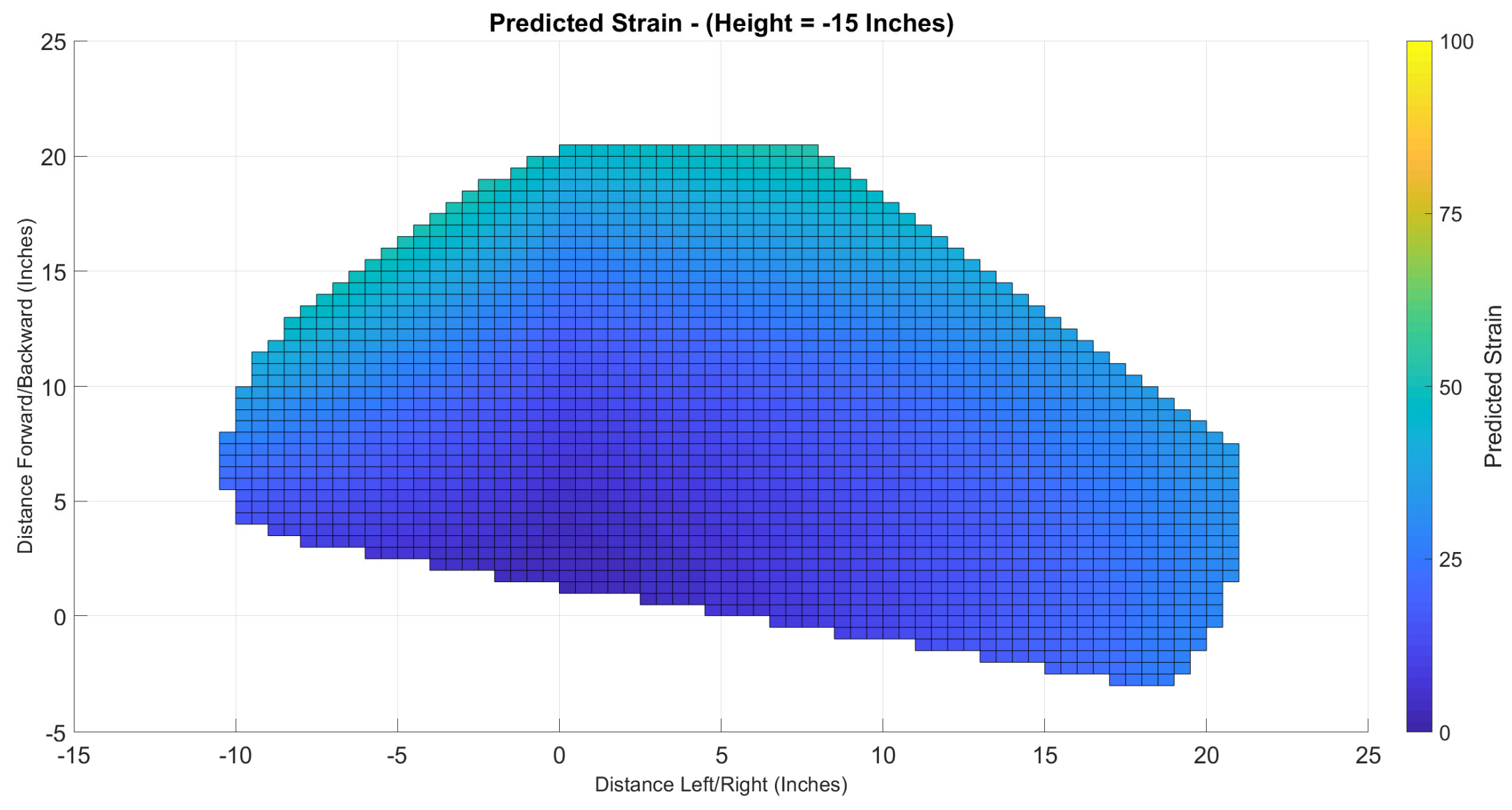


Appendix K9. Predicted Strain with the Load 20 Inches Below Sternal Notch.

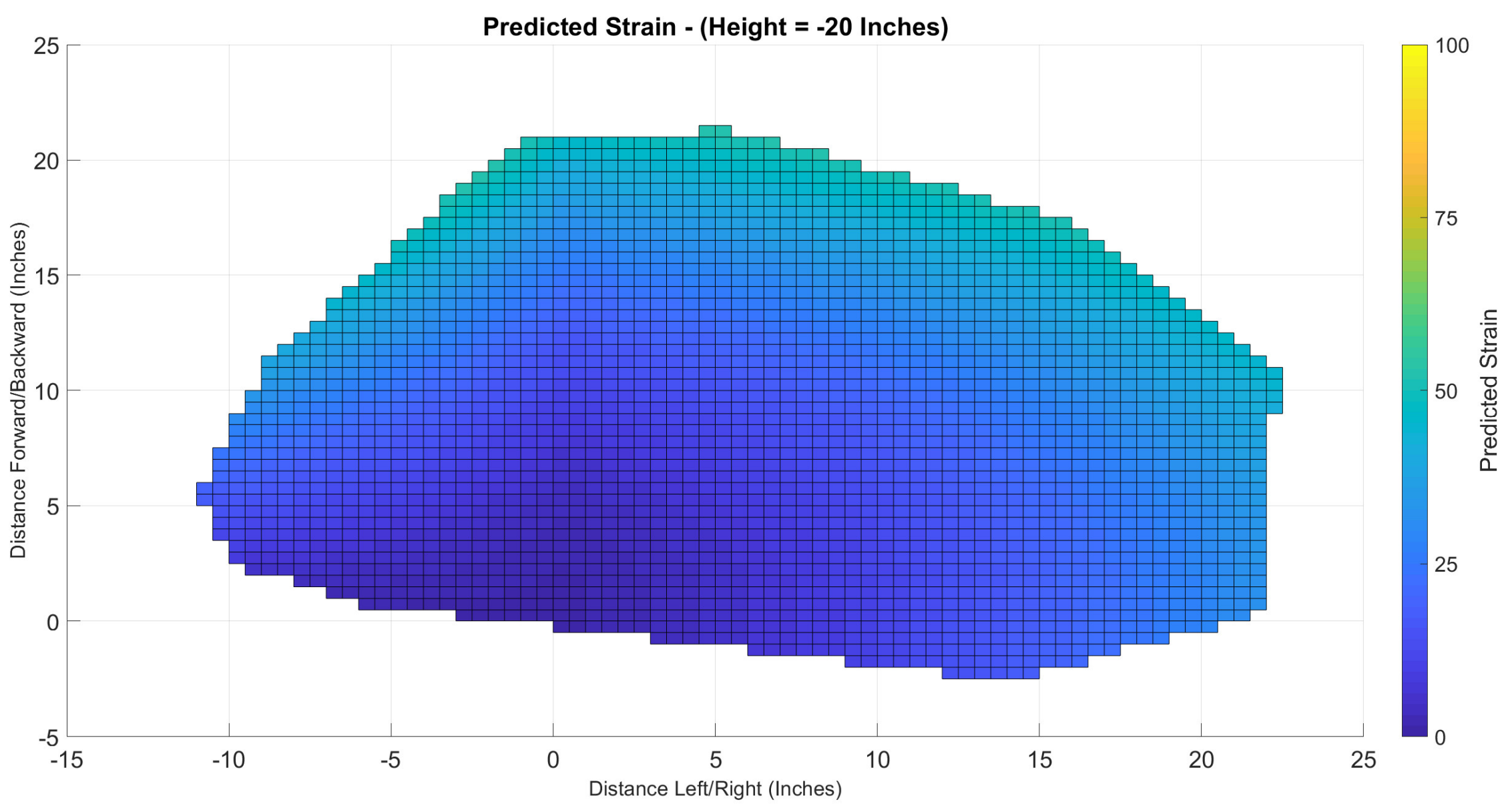




\title{
Appendix L: Specific Aim 3 Consent Form
}

\section{WestViriginiaUniversity.}

Human Research Protocol

Only Minimal Risk Consent Form

Without HIPAA

\author{
Only Minimal Risk \\ Consent Information Form (without HIPAA) \\ Principal Investigator \\ Dr. Ashish Nimbarte \\ Department \\ Industrial and Management Systems Engineering \\ Protocol Number \\ \#1802986207 \\ Study Title \\ Development of a Task Analysis Tool to Estimate Shoulder Strain During a \\ Lifting Task: Part III - Validation of the Task Analysis Tool \\ Co-Investigator(s) \\ Christopher Moore
}

\section{Contact Persons}

In the event you experience any side effects or injury related to this research, you should contact Dr. Ashish Nimbarte at (304) 293-9473 or Christopher Moore at (304) 685-5532. (After hours contact: Dr. Ashish Nimbarte at (225) 226-8813). If you have any questions, concerns, or complaints about this research, you can contact Christopher Moore at (304) 6855532.

For information regarding your rights as a research subject, to discuss problems, concerns, or suggestions related to the research, to obtain information or offer input about the research, contact the Office of Research Integrity and Compliance at (304) 293-7073.

In addition if you would like to discuss problems, concerns, have suggestions related to research, or would like to offer input about the research, contact the Office of Research Integrity and Compliance at 304-293-7073.

\section{Introduction}

You, __ have been asked to participate in this research study, which has been explained to you by Christopher Moore. This study is being conducted by Christopher Moore and Dr. Ashish Nimbarte in the Department of Industrial and Management Systems Engineering at West Virginia University.

\section{Purpose(s) of the Study}

In previous research, predictive equations were developed to estimate the relative risk of shoulder strain when lifting an item based on the location of that item in relation to the top of the sternum. The resulting range of values from the equations were separated into low, moderate, and high relative risk ranges. This research tests the hypothesis that, during an unobstructed lift/move of an item between two locations in the reach range, the relative risk will be highest at either the origin or destination and not somewhere during the lift, which would validate this for use during lifting/moving tasks and not just stationary/isometric lifts.

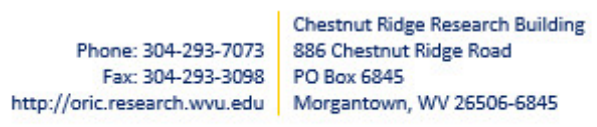

Approved:2-Apr-2018Expires:1-Apr-2019Number:1802986207

$$
\text { Page | } 1
$$

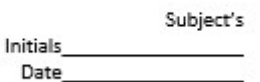




\title{
W. WestVirginiaUniversity.
}

\author{
Human Research Protocol \\ Only Minimal Risk Consent Form \\ Without HIPAA
}

\section{Description of Procedures}

This study involves twelve trials standing in one spot and, using only your right arm, moving an item between two shelves. A two minute rest period will be provided between trials. Prior to completing the trials, a set of 32 retroreflective markers will be affixed to the upper body using double-sided tape. While performing the lifting tasks, these markers will be recorded/tracked with an optical motion capture system utilizing infrared light. After each of the12 lifting tasks, you will be asked to rate your perceived exertion on a scale of $0-10$, where 0 is "Nothing at all" and 10 is "Impossible". Completion of the study takes approximately an hour.

\section{Discomforts}

There are no known or expected risks from participating in this study. Delayed onset muscle soreness is possible, but unlikely, and should subside within 24 hours. Double sided tape will be used to attach markers to skin, so allergic reaction is possible for those with a sensitivity to adhesives.

\section{Alternatives}

You do not have to participate in this study.

\section{Benefits}

You may not receive any direct benefit from this study. The knowledge gained from this study may eventually benefit others. If you are a WVU student, you will not receive any extra credit for participation.

\section{Financial Considerations}

There are no special fees for participating in this study. You will be required to provide a photo identification to receive compensation for participating.

You will be paid $\$ 40$ for completion of the study. If you withdraw before the end of the study, no payment will be made. You will not earn extra credit for participating in this study.

\section{Voluntary Compensation}

If you are injured as a result of this research, treatment will be available. Responsibility for this treatment will be borne by you. In the event that you are physically injured as a result of participating in this research, care will be available. You will, however, be responsible for the charges for the care. There is no commitment to provide any compensation for research-related injury. You should realize, however, that you have not released this institution from liability for negligence. Please contact the investigator, Dr. Ashish Nimbarte at (304) 293-9473 if you are injured or for further information.

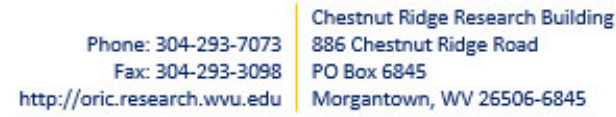

Approved:2-Apr-2018Expires:1-Apr-2019Number:1802986207 


\section{W-WestVirginiaUniversity.}

Human Research Protocol

Only Minimal Risk Consent Form

Without HIPAA

\section{Confidentiality}

Any information about you that is obtained as a result of your participation in this research will be kept as confidential as legally possible. Your research records and test results, just like hospital records, may be subpoenaed by court order or may be inspected by the study sponsor or federal regulatory authorities (including the FDA if applicable) without your additional consent.

In addition, there are certain instances where the researcher is legally required to give information to the appropriate authorities. These would include mandatory reporting of infectious diseases, mandatory reporting of information about behavior that is imminently dangerous to your child or to others, such as suicide, child abuse, etc.

Audiotapes or videotapes will be kept locked up and will be destroyed as soon as possible after the research is finished. In any publications that result from this research, neither your name nor any information from which you might be identified will be published without your consent.

\section{Voluntary Participation}

Participation in this study is voluntary. You are free to withdraw your consent to participate in this study at any time.

Refusal to participate or withdrawal will not affect [your class standing or grades, as appropriate] and will involve no penalty to you. Refusal to participate or withdrawal will not affect your future care, or [your employee status, as appropriate] at West Virginia University.

In the event new information becomes available that may affect your willingness to participate in this study, this information will be given to you so that you can make an informed decision about whether or not to continue your participation.

You have been given the opportunity to ask questions about the research, and you have received answers concerning areas you did not understand.

Upon signing this form, you will receive a copy.

I willingly consent to participate in this research.

\section{Signatures}

Signature of Subject

\begin{tabular}{|c|c|c|c|c|}
\hline \multicolumn{2}{|l|}{ Printed Name } & \multirow[t]{2}{*}{ Date } & \multicolumn{2}{|l|}{ Time } \\
\hline Phone: $304-293-7073$ & $\begin{array}{l}\text { Chestnut Ridge Research Building } \\
886 \text { Chestnut Ridge Road }\end{array}$ & & & $P a g \in \mid 3$ \\
\hline Fax. $304-293-3098$ & PO Box 6845 & & & Subject's \\
\hline http://oric.research.wvu.edu & Morgantown, WV 26506-6845 & & $\begin{array}{l}\text { Initials_ } \\
\text { Date_ }\end{array}$ & \\
\hline
\end{tabular}

Approved:2-Apr-2018Expires:1-Apr-2019Number:1802986207 
The participant has had the opportunity to have questions addressed. The participant willingly agrees to be in the study.

Signature of Investigator or Co-Investigator

Printed Name

Date

Time

\footnotetext{
Chestnut Ridge Research Building

886 Chestnut Ridge Road

\begin{tabular}{c|c|c} 
Phone: $304-293-7073$ & 886 Chestnut \\
Fax $304-293-3098$ & PO Box 6845
\end{tabular}

http://oric.research.wvu.edu Morgantown, WV 26506-6845
}

Page |4

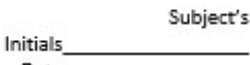

Date

Approved:2-Apr-2018Expires:1-Apr-2019Number:1802986207 